\title{
Uma Introdução aos Pequenos Cardinais \\ e às suas : Aplicações em Topologia
}

Samuel Gomes da Silva

$$
\begin{gathered}
\text { DISSERTAÇÃO APRESENTADA } \\
\text { AO } \\
\text { INSTITUTO DE MATEMÁTICA E ESTATÍSTICA }
\end{gathered}
$$$$
\text { DA }
$$$$
\text { UNIVERSIDADE DE SÃO PAULO }
$$$$
\text { PARA }
$$$$
\text { OBTENÇÃO DO GRAU DE MESTRE }
$$$$
\text { EM }
$$$$
\text { MATEMÁTICA }
$$

Área de Concentração: Topologia Geral e Conjuntística Orientador: Prof. Dr. Stavros Christodoulou

Durante a elaboração deste trabalho o autor recebeu apoio financeiro do CNPq

-São Paulo, julho de 1998- 


\title{
Uma Introdução aos Pequenos Cardinais \\ e às suas Aplicações em Topologia
}

\author{
Este exemplar corresponde à redação \\ final da dissertação devidamente corrigida \\ e defendida por Samuel Gomes da Silva \\ e que foi aprovada pela comissão julgadora.
}

São Paulo, 24 de julho de 1998.

Comissão Julgadora:

- Prof. Dr. Stavros Christodoulou (orientador) - IME-USP

- Profa Dra - Ofélia Teresa Alas - IME-USP

- Prof. Dr. Francisco Antonio Dória - CFCH-UFRJ 
Aos meus amigos. 


\section{Agradecimentos}

Ao meu orientador, Prof. Stavros Christodoulou, pela confiança e pela coragem de orientar um aluno egresso da Licenciatura. Com sua ajuda, consegui sanar muitas deficiências e amadurecer bastante. Será sempre um grande amigo e companheiro de pesquisa, e também, é claro, será sempre alguém com quem dividir experiências sobre problemas de voz e balas de própolis.

Aos professores do grupo de pesquisa em Topologia Conjuntística do IME/USP com quem travei contato: à profa. Lúcia Junqueira, pelo curso de Topologia na pós e pelas quentíssimas dicas bibliográficas, e ao prof. Artur Tomita, pelo ritmo imposto ao Seminário "Tópicos de Topologia Conjuntística", o qual me fez concluir mais rapidamente que "set theoretic topology é a minha praia".

Ao prof. Stephen Watson, por ter percebido que colei errado a etiqueta ("the label") no meu disquete.

Aos professores do IME/USP que me ajudaram a ingressar no Mestrado: Roberto Costa, que foi o primeiro a conversar seriamente comigo a esse respeito, numa distante noite de 1992. Paulo Cordaro, que me convenceu que "o sonho era possível". Jorge Aragona, por sempre acreditar em mim (até mais do que eu, às vezes), pela ajuda no Exame de Funções Analíticas e pelos "papos matemáticos" nos corredores do Instituto. Francisco Rui, por ser sempre simpático e atencioso. Flávio Coelho, por ser, paradoxalmente, um são-paulino que sempre ajudou este corintiano (pelo menos, com certeza sempre votamos no mesmo partido.). Aos professores Sérgio Alves e Cristina Barufi, sou muito grato pelos tempos em que fui monitor dos cursos de Geometria da Licenciatura Noturno. Ao prof. Sérgio Alves, em especial, devo o primeiro convite para atuar na monitoria.

Ao prof. Nílson José Machado, agradeço a amizade, o respeito e as portas de sua sala sempre abertas.

À profa. Maria Lúcia Sobral Singer, agradeço a atenção a mim dispensada na época em que era minha orientadora de programa.

A luta contra o Latex mereceria um capítulo de agradecimentos. Sou muito grato ao Marcelo Dias Passos, o Major, que mui gentilmente cedeu-me os arquivos de sua dissertação para que eu conhecesse "o que é o Latex". Irene, Zé, Cecília e Jorge foram 
excelentes companheiros na batalha da digitação e no conhecimento do inimigo. Aos mais experientes nessa luta, como Daniel, Fernando, Raul, Paulo e Claus, agradeço pelos toques ou por simplesmente me acalmarem de vez em quando.

Agradeço muito a todos os meus amigos da pós-graduação, especialmente aos mais próximos - mais especificamente, os subscribers da dmacedo-galera@ime.usp.br -, pelo companheirismo e também por me acharem "maluco" e "psicopata" no que se refere ao estudo. Sem esses comentários jocosos e brincadeiras bem-humoradas, não sei daonde arranjaria forças para os longos finais de semana, na época de cumprimento de créditos, ou, pior ainda, para os terríveis janeiro e fevereiro de 97 , quando me preparei para os Exames de Qualificação. Muito obrigado, mesmo.

Aos meus amigos da graduação, o pessoal da Licenciatura Noturno, agradeço a amizade, o carinho, a confiança, e as constantes presença e torcida durante todos esses anos, desde 1991.

Aos amigos da Estação Ciência, agradeço por terem deixado a USP e toda a cidade de São Paulo repletas de lugares tão aconchegantes quanto a minha própria casa.

Aos amigos do colégio Anhangüera, agradeço por terem sido testemunhas na formação da pessoa que sou hoje. "Não sou nem sombra do que já fui e nem metade do que queria ser, mas pelo menos morrerei sabendo que tentei".

Aos amigos da empresa Wema, com quem aprendi muito a respeito de responsabilidade, organização e relações interpessoais - o que é grande parte do chamado "aprender a viver em sociedade".

Aos amigos do bom e velho ginásio no Retiro, agradeço por terem crescido junto comigo.

Aos amigos professores e alunos da EEPG e CEFAM Experimental da Lapa, agradeço pelo tanto que aprendi e pelo tanto que me senti querido nos anos em que ali trabalhei. Alguns professores devem passar a vida sem ter o prazer de terem sido paraninfos; ali, o fui por duas gratas vezes.

Um agradecimento especial, e infelizmente póstumo, ao Prof. Seiji Hariki, que me ajudou muito na escolha dos caminhos a seguir quando terminei a graduação. Que sua dedicação e sua luta pela causa da educação matemática continuem inspirando a mim e a muitos outros.

À minha família, uma expectativa de melhoria de vida. 
Sejam bem vindos a uma pequena parte do paraíso que Cantor criou para nós. 


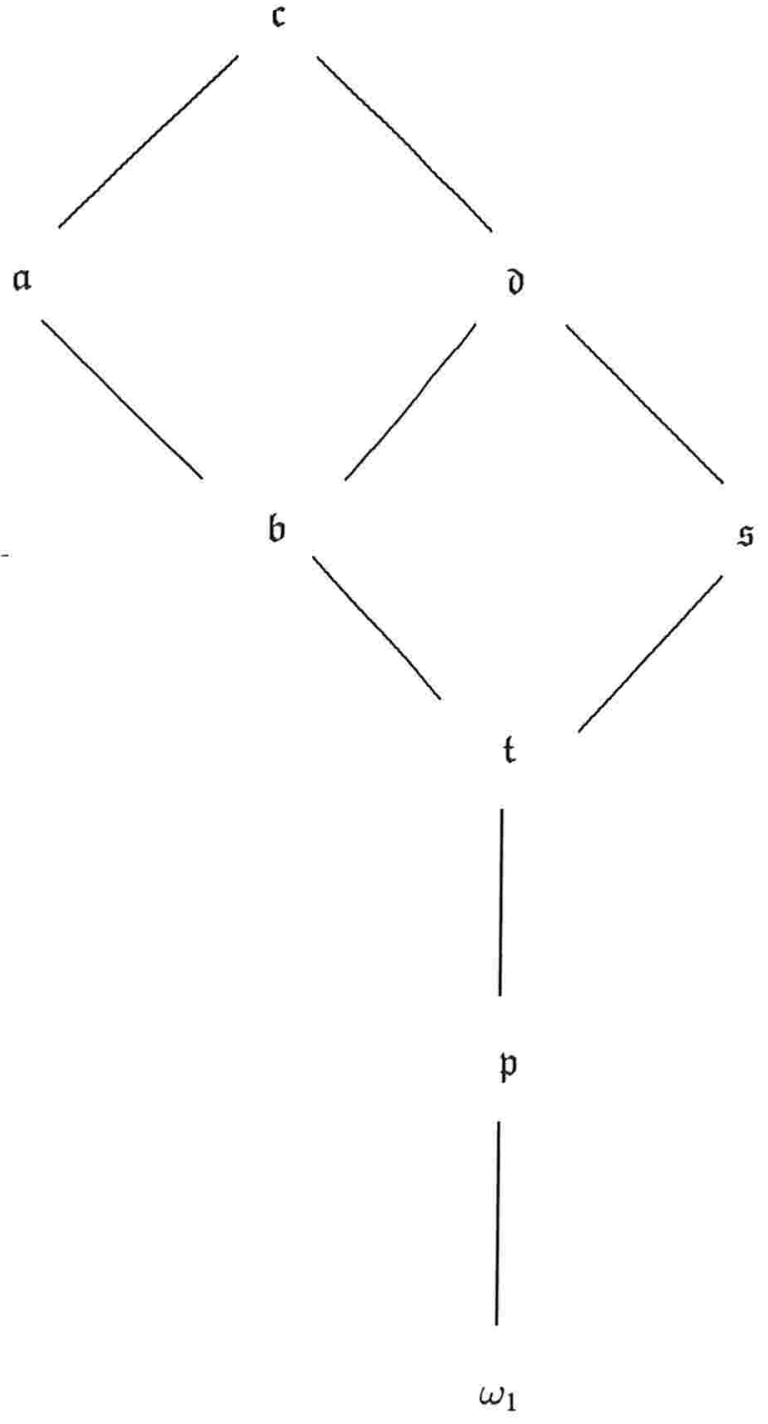




\section{Resumo}

Este trabalho estuda, do ponto de vista conjuntístico e topológico, alguns dos chamados pequenos cardinais. Definimos seis desses cardinais $(\mathfrak{a}, \mathfrak{b}, \mathfrak{o}, \mathfrak{p}, \mathfrak{s}$ e $\mathfrak{t})$ e apresentamos alguns resultados válidos em ZFC a respeito deles; resultados (e problemas abertos) de consistência e independência são comentados. Elementos básicos da teoria de gaps são revisitados. Finalmente, algumas aplicações dos nossos pequenos cardinais à Topologia (com destaque para a influência desses cardinais na existência - ou não - de espaços topológicos que satisfaçam determinadas propriedades) são detalhadamente expostas.

\footnotetext{
Abstract

This work studies some of the called small cardinals by a set-theoretical and topological view. We define six of these cardinals $(\mathfrak{a}, \mathfrak{b}, \mathfrak{o}, \mathfrak{p}, \mathfrak{s}$ and $\mathfrak{t}$ ) and also present some ZFC results about them. Some consistency and independence results as well as open problems are commented. Basic elements of gap theory are revisited. Finally, some applications of our small cardinals in Topology (pointing out the influence of such cardinals on the existence of topological spaces which satisfy some properties) are exposed with details.
} 


\section{Sumário}

Introdução $\quad$ iii

1 Preliminares Conjuntísticas

e Topológicas 1

1.1 Teoria dos Conjuntos . . . . . . . . . . . . . . . . . . 1

1.2 Topologia Geral . . . . . . . . . . . . . . . . . . 8

2 Seis pequenos cardinais $\quad 16$

2.1 Apresentação dos seis pequenos cardinais . . . . . . . . . . . . . . 16

$2.2 \mathfrak{a}, \mathfrak{b}, \mathfrak{d}, \mathfrak{p}, \mathfrak{s}$ e $t$ em $Z F C \ldots \ldots \ldots \ldots \ldots 22$

2.3 Definições alternativas para os pequenos cardinais . . . . . . . . . . . . . . . . 34

2.4 Resultados de consistência e de independência . . . . . . . . . . . . 45

Notas . . . . . . . . . . . . . . . . . . . . . . 49

3 Gaps em $\left({ }^{\omega} \omega,<^{*}\right)$ e $\left(\mathcal{P}(\omega), \subset^{*}\right) \quad 51$

3.1 Gaps em conjuntos parcialmente ordenados . . . . . . . . . . . . . . 51

3.2 Gaps em $\left({ }^{\omega} \omega,<^{*}\right)$ e $\left(\mathcal{P}(\omega), \subset^{*}\right) \ldots \ldots \ldots \ldots \ldots$

3.3 O cardinal $\omega_{1}$ e os gaps de Luzin e de Hausdorff . . . . . . . . . . . . 62

Notas .............................. 69

4 Algumas Aplicações à Topologia $\quad 70$

4.1 Espaços seqüencialmente compactos,

enumeravelmente compactos e correlatos . . . . . . . . . . . 70 
4.1.1 Peso, caráter e cardinalidade de (enumeravelmente)

compactos não seqüencialmente compactos . . . . . . . . . . 70

4.1.2 Produtos. Problemas do tipo de Scarborough e Stone . . . . . . . . 77

4.1.3 Espaços de $C^{*}$-boas-ordens e espaços de gaps . . . . . . . . . . . . 81

4.1.4 Tight gaps, big gaps, os cardinais $\mathfrak{p}$ e $\mathfrak{b}$

e os espaços de $\left(\omega_{1}, \omega_{1}^{*}\right)$-gaps . . . . . . . . . . . . 87

4.1.5 FR-espaços e alguns resultados de Nyikos e Vaughan . . . . . . . 95

4.2 O cardinal $\mathfrak{d}$ e a cobertura dos

irracionais por compactos . . . . . . . . . . . . . . 102

4.3 A normalidade do produto de um espaço de

Lindelöf com os irracionais e o cardinal b . . . . . . . . . . . . . 105

4.4 Considerações finais . . . . . . . . . . . . . . . . . . . . 112

$\begin{array}{ll}\text { Bibliografia } & 113\end{array}$

Índice 


\section{Introdução}

Um pequeno cardinal é um cardinal que é definido a partir da cardinalidade de um conjunto de alguma forma associado ao conjunto $\omega$ dos números naturais ([Vau96]). Assim, $\aleph_{0}$ e $2^{\aleph_{0}}=\mathfrak{c}$ são pequenos cardinais. Neste trabalho, consideraremos pequenos cardinais (ou invariantes cardinais do continuum, como também são chamados em alguns textos) definidos, em geral, da seguinte forma: dada uma propriedade $Q$ que se aplica a subfamílias de $[\omega]^{\omega}$ ou de ${ }^{\omega} \omega$, associamos a ela um invariante cardinal $\lambda$ dado por $\lambda=\min \{|\mathcal{Q}|$ : $\mathcal{Q} \subseteq[\omega]^{\omega}$ satisfaz $\left.Q\right\}$ (ou $\lambda=\min \left\{|\mathcal{Q}|: \mathcal{Q} \subseteq{ }^{\omega} \omega\right.$ satisfaz $\left.Q\right\}$, conforme o caso.). As propriedades que definem os seis pequenos cardinais com os quais trabalharemos $-\mathfrak{a}, \mathfrak{b}, \mathfrak{o}$, $\mathfrak{p}, \mathfrak{s}$ e $\mathfrak{t}$ - estão enunciadas em termos puramente conjuntísticos, o que facilita a obtenção de resultados de consistência a respeito desses cardinais. No entanto, esses cardinais "surgem inesperadamente" em muitos outros contextos; estudaremos aqui, com grande interesse, questões topológicas nas quais esses cardinais atuam. A principal referência a respeito de pequenos cardinais é o artigo The Integers and Topology ([vD84]), de autoria de Erick K. van Douwen e publicado em 1984 no Handbook of Set Theoretic Topology; a partir desse artigo iniciamos a pesquisa que resultou nesta dissertação.

Cada um dos seis pequenos cardinais de que trataremos é não-enumerável e limitado superiormente por $\mathfrak{c}$; logo, sob a Hipótese do Contínuo vale " $\omega_{1}=\kappa=\mathfrak{c}$ " para $\kappa \in\{\mathfrak{a}$, $\mathfrak{b}, \mathfrak{d}, \mathfrak{p}, \mathfrak{s}, \mathfrak{t}\}$. Porém, além de " $\omega_{1}=\kappa=\mathfrak{c}$ ", qualquer uma das asserções " $\omega_{1}=\kappa<\mathfrak{c}$ ", " $\omega_{1}<\kappa<\mathfrak{c}$ " e " $\omega_{1}<\kappa=\mathfrak{c}$ " é consistente com ZFC. Em particular, " $\omega_{1}=\kappa$ " e " $\kappa=\mathfrak{c} "$ (para $\kappa \in\{\mathfrak{a}, \mathfrak{b}, \mathfrak{d}, \mathfrak{p} ; \mathfrak{s}, \mathfrak{t}\}$ ) são hipóteses mais fracas do que a Hipótese do Contínuo. Existem, inclusive, registros em nossa literatura de resultados originariamente obtidos a partir da Hipótese do Contínuo ( $\omega_{1}=\mathfrak{c}$ ") mas que, na verdade, foram essencialmente obtidos a partir de alguma hipótese equivalente a uma asserção do tipo " $\omega_{1}=\kappa$ " ou " $\kappa=\mathfrak{c}$ " para algum $\kappa$ entre nossos pequenos cardinais. 
O primeiro capítulo deste trabalho apenas lista as "ferramentas" conjuntísticas e topológicas que serão utilizadas no restante do texto. No segundo capítulo, os seis cardinais $\mathfrak{a}, \mathfrak{b}, \mathfrak{d}, \mathfrak{p}, \mathfrak{s}$ e $\mathfrak{t}$ são cuidadosamente definidos e alguns resultados básicos sobre eles (válidos em $Z F C$ ) são apresentados. Em seguida, cada um dos cardinais recebe "definições alternativas", que são caracterizações desses cardinais enunciadas de modo distinto da caracterização "standard" dada inicialmente. O capítulo é encerrado com alguns comentários a respeito de resultados de consistência e independência, com destaque para " $M A \Rightarrow \mathfrak{p}=\mathfrak{c}$ ".

No terceiro capítulo, estudamos os gaps em $\left\langle\mathcal{P}(\omega), \subset^{*}\right\rangle$ e $\left\langle{ }^{\omega} \omega,<^{*}\right\rangle$ e as relações existentes entre esses gaps e pequenos cardinais. Muitos resultados a respeito de pequenos cardinais podem ser traduzidos para a linguagem de gaps, e em alguns casos esses resultados são até mesmo melhor compreendidos nessa linguagem. Apresentamos, também no terceiro capítulo, uma demonstração de um célebre teorema de Hausdorff, publicado em 1936 ([Hau36]), que garante a existência de $\left\langle\omega_{1}, \omega_{1}^{*}\right\rangle$-gaps em $\left\langle\mathcal{P}(\omega), \subset^{*}\right\rangle$. Gaps desse tipo serão usados na constrúção de interessantes exemplos de espaços topológicos, no quarto e último capítulo deste trabalho.

O quarto capítulo é dedicado a aplicações da teoria de pequenos cardinais à Topologia. Basicamente, estudaremos a influência dos pequenos cardinais em questões topológicas relacionadas a determinadas classes de espaços, como espaços compactos, espaços enumeravelmente compactos e espaços seqüencialmente compactos. O espaço completamente metrizável $\mathbb{P}$ dos números irracionais também é estudado com destaque nesse capítulo, e veremos que a existência de um espaço de Lindelöf cujo produto com $\mathbb{P}$ é não-normal está intimamente ligada a uma hipótese a respeito do cardinal b. 


\section{Capítulo 1}

\section{Preliminares Conjuntísticas e Topológicas}

Neste capítulo apresentaremos (de forma resumida) algumas definições e resultados básicos (em Teoria dos Conjuntos e Topologia Geral) que serão utilizados em todo o restante do texto.

\subsection{Teoria dos Conjuntos}

Utilizaremos o sistema axiomático de Zermelo-Fraenkel com o Axioma da Escolha, tradicionalmente denominado " $Z F C$ ". Nossa referência principal é o livro de Kunen ([Kun80]). O livro de Jech ([Jec78]) é excelente no que se refere à Aritmética Cardinal e o recente livro de Just e Weese ([JW97]) é bastante moderno (no que se refere aos temas abordados) e interessante.

Operações básicas sobre conjuntos e noções relativas ao conceito de ordem (relações, ordens parciais e totais, boas-ordens e isomorfismos entre boas-ordens, segmentos iniciais, elementos maximais e minimais, máximos e mínimos, supremos e ínfimos) serão supostos conhecidos. A diferença entre dois conjuntos $A$ e $B$ será denotada por $A \backslash B$. $\mathrm{O}$ conjunto de todos os subconjuntos de um conjunto $A$ será denotado por $\mathcal{P}(A)$. Como em qualquer texto fortemente baseado em teoria dos conjuntos, relações e funções serão 
consideradas como conjuntos de pares ordenados.

Um conjunto $A$ é dito transitivo se todo elemento de $A$ é também subconjunto de $A$. Um conjunto é dito um ordinal se for transitivo e bem-ordenado por $\in$. Com isso, todos os elementos de um ordinal são também ordinais. Ordinais serão sempre designados por letras gregas minúsculas: $\alpha, \beta, \gamma, \delta, \xi, \eta, \zeta$, etc. Freqüentemente escreveremos " $\alpha<\beta$ " ao invés de " $\alpha \in \beta$ ". Assim, para todo ordinal $\alpha$ tem-se $\alpha=\{\beta: \beta<\alpha\}$, isto é, um ordinal $\alpha$ é o conjunto formado por todos os ordinais menores do que $\alpha$.

Dada uma boa-ordem $\langle A, R\rangle$, existe um único ordinal $\alpha$ tal que $\langle A, R\rangle$ e $\langle\alpha, \in\rangle$ são isomorfas. Tal ordinal $\alpha$ é chamado de tipo de ordem de $\langle A, R\rangle$.

Dado um ordinal $\alpha$, definimos o sucessor de $\alpha$, denotado por $S(\alpha)$ ou $\alpha+1$, pondo $\alpha+1=S(\alpha)=\alpha \cup\{\alpha\}$. S( $\alpha)$ é um ordinal. Um ordinal $\alpha$ é dito um ordinal sucessor se existir $\beta$ para o qual $\alpha=S(\beta)$. Em particular, tem-se $0=\emptyset, 1=S(0)=\{0\}$, $2=S(1)=\{0,1\}$, etc. Um ordinal $\alpha \neq 0$ que não é ordinal sucessor é dito um ordinal limite. O menor ordinal limite é $\omega=\{0,1,2, \ldots\}$, o conjunto de todos os números naturais. Números naturais serão designados por letras minúsculas: i, j, k, l, m, n, etc.

Todo conjunto não-vazio de ordinais admite um mínimo, que é dado pela intersecção do conjunto. Todo conjunto de ordinais admite um supremo, que é dado pela união do conjunto. Assim, se $A$ é um conjunto de ordinais, $\sup (A)=\bigcup A$, que é o menor limitante superior de $A$, e, no caso de $A \neq \emptyset, \min (A)=\bigcap A$.

Processos de indução e recursão serão feitos em qualquer conjunto bem-ordenado. Se o tipo de ordem do conjunto em questão for $\leqslant \omega$ (respectivamente, $>\omega$ ), diremos que a indução (ou recursão) é finita (respectivamente, transfinita).

Se $A$ e $B$ são conjuntos, denotamos por ${ }^{A} B$ o conjunto das funções de $A$ em $B$, isto é, ${ }^{A} B=\{f: f$ é função, $\operatorname{dom}(f)=A$ e $i m(f) \subseteq B\}$ (em particular, ${ }^{\omega} \omega$ é conjunto de todas as funções de $\omega$ em $\omega)$. Dados $f: X \mapsto Y, A \subseteq X$ e $B \subseteq Y, f[A]$ e $f^{-1}[B]$ denotam respectivamente a imagem de $A$ por $f$ e a pré-imagem de $B$ por $f$. Se $\operatorname{dom}(f)=\alpha$ para algum ordinal $\alpha$, freqüentemente escrevemos $f=\left\langle f_{\xi}: \xi<\alpha\right\rangle$ (onde, obviamente, $f_{\xi}=f(\xi)$ para todo $\left.\xi<\alpha\right)$ e $f$ é dita uma seqüência de comprimento $\alpha$. No caso $\alpha=\omega$, $f$ é dita simplesmente uma seqüência.

O símbolo "†" terá dois significados neste texto: se $f$ é uma função e $A \subseteq \operatorname{dom}(f)$, $f \uparrow A=\{\langle x, f(x)\rangle: x \in A\}$ que é a restrição de $f$ a $A$. Se $\mathcal{F}$ é uma família de conjuntos e $A$ é um conjunto, $\mathcal{F} \uparrow A$ denota a família $\mathcal{F} \uparrow A=\{F \cap A: F \in \mathcal{F}\}$. Sempre estará 
claro no contexto em questão qual é o significado desejado para "†".

Se $A$ e $B$ são conjuntos, dizemos que $A$ e $B$ são equipotentes (e denotamos isso por $A \approx B$ ) se existir $f: A \mapsto B$ bijetora. Sob $A C$ (Axioma da Escolha) todo conjunto $A$ pode ser bem-ordenado, donde $\{\alpha: \alpha \approx A\}$ é sempre um conjunto não-vazio de ordinais. Definimos então a cardinalidade de um conjunto $A$ pondo $|A|=\min \{\alpha: \alpha \approx A\}$. Um ordinal $\alpha$ é dito um cardinal se $|\alpha|=\alpha$. Assim, $\alpha$ é um cardinal se e só se $\alpha$ não é equipotente a nenhum ordinal menor que $\alpha$. Usaremos as letras $\kappa, \lambda, \mu, \theta$ para designar cardinais.

Se $X$ é um conjunto e $\lambda$ é um cardinal, definimos

$$
[X]^{\lambda}=\{A \in \mathcal{P}(X):|A|=\lambda\}
$$

$\mathrm{e}$

$$
[X]^{<\lambda}=\{A \in \mathcal{P}(X):|A|<\lambda\}
$$

Em particular, $[\omega]^{\omega}$ é o conjunto de todos os subconjuntos infinitos de $\omega$ e $[\omega]^{<\omega}$ é o conjunto de todos os subconjuntos finitos de $\omega$.

Assumiremos conhecidas as definições de soma, produto e potência de ordinais ("aritmética ordinal") e soma, produto e potência de cardinais("aritmética cardinal"). Também são assumidos conhecidos os resultados: $\left|[\omega]^{<\omega}\right|=\omega,|\mathcal{P}(\omega)|=\left|[\omega]^{\omega}\right|=\left|{ }^{\omega} \omega\right|=$ $2^{\omega}$. O cardinal $2^{\omega}$ será em geral denotado por "c", o que já é tradicional.

Dado um cardinal $\kappa$, denotamos por $\kappa^{+}$o cardinal sucessor de $\kappa$, que é o menor cardinal estritamente maior que $\kappa$. Um cardinal $\kappa$ é dito um cardinal sucessor se existir um cardinal $\lambda$ para o qual $\kappa=\lambda^{+}$. Um cardinal $\kappa$ é dito um cardinal limite se $\kappa>\omega$ e $\kappa$ não é um cardinal sucessor. . Considerando-se a "hierarquia" dos cardinais infinitos dada por

(1) $\aleph_{0}=\omega_{0}=\omega$

(2) $\aleph_{\alpha+1}=\omega_{\alpha+1}=\left(\omega_{\alpha}\right)^{+}$

(3) Para $\gamma$ limite, $\aleph_{\gamma}=\omega_{\gamma}=\sup \left\{\omega_{\xi}: \xi<\gamma\right\}$

temos para todo $\alpha$ que o cardinal $\omega_{\alpha}$ é cardinal sucessor ou limite conforme $\alpha$ seja um ordinal sucessor ou limite. Todo cardinal infinito $\kappa$ é da forma $\kappa=\omega_{\alpha}$ para algum $\alpha$. É claro também que cardinais infinitos são ordinais limite. 
O célebre Teorema de Cantor nos dá que, para qualquer conjunto $X,|X|<|\mathcal{P}(X)|$. Em particular, $\omega<2^{\omega}$, isto é, $\omega_{1} \leqslant 2^{\omega}$ (aritmética cardinal). Mais geralmente, $\omega_{\alpha+1} \leqslant 2^{\omega_{\alpha}}$ para todo ordinal $\alpha$. A Hipótese do Contínuo ( "CH") é a asserção $2^{\omega}=\omega_{1}$. A Hipótese Generalizada do Contínuo ("GCH") é a asserção: para todo ordinal $\alpha, 2^{\omega_{\alpha}}=\omega_{\alpha+1}$.

Sejam $\alpha, \beta$ ordinais. Se $\beta$ é ordinal sucessor, $\beta=\zeta+1$ digamos, uma função $f: \alpha \mapsto \beta$ é dita cofinal em $\beta$ se $\zeta \in i m(f)$. Se $\beta$ é ordinal limite, $f: \alpha \mapsto \beta$ é dita cofinal em $\beta$ se $i m(f)$ é ilimitada em $\beta$ (i.e., $\sup (i m(f))=\beta$ ). A cofinalidade de um ordinal $\beta$ (denotada por $c f(\beta)$ ) é o menor ordinal $\alpha$ para o qual existe $f: \alpha \mapsto \beta$ cofinal. Se $\beta$ é ordinal sucessor, $\operatorname{cf}(\beta)=1$, logo só existe interesse nas cofinalidades de ordinais limite. Se $\beta$ é limite e $c f(\beta)=\alpha$, podemos afirmar que existe $f: \alpha \mapsto \beta$ cofinal e estritamente crescente.

Um ordinal limite $\beta$ é dito regular se $c f(\beta)=\beta$, caso contrário é dito singular. Um cardinal infinito $\kappa$ é dito regular (respectivamente, singular) se o ordinal limite $\kappa$ for regular (respectivamente, singular).

Valem os seguintes fatos a respeito de cofinalidade:

(i) $c f(\beta)$ é regular para todo $\beta$ limite;

(ii) Se $\alpha$ é regular, então $\alpha$ é cardinal (em particular, cofinalidades de ordinais limite são sempre cardinais regulares);

(iii) $\omega$ é regular;

(iv) Para todo cardinal infinito $\kappa, \kappa^{+}$é regular.

$(v)$ Para todo cardinal infinito $\kappa, \kappa<c f\left(2^{\kappa}\right)$.

Observamos que a seguinte definição de cofinalidade é equivalente à que demos: seja $\beta$ um ordinal limite. $A \subseteq \beta$ é dito cofinal em $\beta$ se $\sup (A)=\beta$. Então $\operatorname{cf}(\beta)=$ $\min \{|A|: A$ é cofinal em $\beta\}$. Logo,

(vi) Se $A \subset \delta$ e $|A|<c f(\delta)$, então $\sup (A)<\delta$; em particular, (vii) Se $\kappa$ é regular e $A \in[\kappa]^{<\kappa}$ então $\sup (A)<\kappa$.

Seja $\delta$ um ordinal limite; $A \subseteq \delta$ é dito fechado em $\delta$ se, para todo ordinal limite $\zeta<\delta, \zeta \in A$ sempre que $\zeta \cap A$ for ilimitado em $\zeta$ (o que equivale a dizer que $A$ é fechado em $\delta$, na topologia da ordem.). $A \subseteq \delta$ é dito c.u.b. em $\delta$ ("closed and unbounded") se $A$ 
é fechado em $\delta$ e $\sup (A)=\delta . S \subseteq \delta$ é dito estacionário em $\delta$ se $S \cap A \neq \emptyset$ para todo $A$ c.u.b. em $\delta$. Destacamos os seguintes resultados a respeito de c.u.b.'s e estacionários:

Lema (6.8 de [Kun80]) Se $\lambda=c f(\delta)>\omega$, então a interseç̧ão de qualquer família de menos do que $\lambda$ c.u.b.'s em $\delta$ é c.u.b. em $\delta$.

Lema de Fodor("Pressing Down Lemma", 6.15 de [Kun80]) Seja $\kappa>\omega u m$ cardinal regular, $S \subseteq \kappa$ estacionário e $f: S \mapsto \kappa$ uma função regressiva (i.e., $(\forall \gamma \in$ $S)[f(\gamma)<\gamma]$ ). Então existe $\alpha<\kappa$ tal que $f^{-1}[\{\alpha\}]$ é estacionário em $\kappa$.

Um conceito central em nosso trabalho será o de pré-ordens. Uma pré-ordem é um par $\left\langle D, \leqslant^{*}\right\rangle$, onde $D$ é um conjunto e $\leqslant^{*}$ é uma relação reflexiva e transitiva. Dados $x$, $y \in D$, a notação " $x<^{*} y$ " denotará " $x \leqslant^{*} y$ e $y \not^{*} x$ " (em particular, $\subset$ sempre denotará inclusão estrita.). Observamos que, nessas condições, " $x<^{*} y$ " é, em geral, mais forte do que " $x \leqslant *$ e $x \neq y$ ", pois, como não vale necessariamente a antissimetria, podemos ter $x, y \in D$ tais que $\dot{x} \neq y, x \leqslant^{*} y$ e $y \leqslant^{*} x$. Uma pré-ordem $\left\langle D, \leqslant^{*}\right\rangle$ pode gerar uma ordem parcial "quocientando-se" o conjunto $D$ pela relação de equivalência " =*", definida naturalmente da seguinte forma: para quaisquer $x, y \in D$,

$$
x={ }^{*} y \Longleftrightarrow\left(x \leqslant{ }^{*} y\right) \wedge\left(y \leqslant{ }^{*} x\right)
$$

e a relação $\leqslant_{D /==^{*}}^{*}$ no quociente de $D$ por $=^{*}$ dada por $[x]_{=^{*}} \leqslant_{D /={ }^{*}}^{*}[y]_{=*} \Leftrightarrow x \leqslant \leqslant^{*} y$ é uma ordem parcial. Freqüentemente assumiremos que uma determinada discussão a respeito de uma pré-ordem está restrita a representantes de classes de equivalência por $=^{*}$, e sob essa interpretação $\leqslant *$ irá se comportar como uma ordem parcial de fato.

Se $\left\langle X, \leqslant^{*}\right\rangle$ é uma pré-ordem, $B \subseteq X$ é dito ilimitado em $\left\langle D, \leqslant^{*}\right\rangle$ (ou, simplesmente, ilimitado) se $(\forall x \in X)(\exists b \in B)\left[b \mathbb{*}^{*} x\right] . D \subseteq X$ é dito cofinal em $\left\langle X, \leqslant^{*}\right\rangle$ (ou, simplesmente, cofinal) se $(\forall x \in X)(\exists d \in D)[x \leqslant * d]$.

Serão duas as pré-ordens mais importantes neste trabalho; a primeira é $\left\langle\mathcal{P}(\omega), \subseteq^{*}\right\rangle$, definida da seguinte maneira: para quaisquer $A, B \subseteq \omega, A \subseteq B \Leftrightarrow A \backslash B$ é finito. Em algumas situações, consideraremos também $\left\langle\mathcal{P}(X), \subseteq^{*}\right\rangle$ para outros conjuntos enumeráveis $X$. A outra pré-ordem é $\left\langle{ }^{\omega} \omega, \leqslant^{*}\right\rangle$, definida da seguinte forma: para quaisquer $f, g \in{ }^{\omega} \omega$, $f \leqslant^{*} g \Leftrightarrow\{n<\omega: g(n)<f(n)\}$ é finito. Para a verificação da transitividade das relações dadas, basta ver que, se $A, B, C \subseteq \omega, f, g, h \in{ }^{\omega_{\omega}}, A \backslash C \subseteq(A \backslash B) \cup(B \backslash C)$ e 
$\{n<\omega: h(n)<f(n)\} \subseteq\{n<\omega: g(n)<f(n)\} \cup\{n<\omega: h(n)<g(n)\}$.

No que se refere à pré-ordem $\left\langle\mathcal{P}(\omega), \subseteq^{*}\right\rangle$, as seguintes propriedades são de fácil verificação e serão utilizadas livremente neste trabalho; para $A, B, C \in \mathcal{P}(\omega)$ quaisquer,

(i) $A={ }^{*} B \Rightarrow A \backslash C={ }^{*} B \backslash C$

(ii) $A={ }^{*} B \Rightarrow C \backslash A={ }^{*} C \backslash B$

(iii) $A={ }^{*} B \Rightarrow A \cup C={ }^{*} B \cup C$

(iv) $A={ }^{*} B \Rightarrow A \cap C={ }^{*} B \cap C$

(Para $(i),(A \backslash C) \backslash(B \backslash C) \subseteq A \backslash B$ e analogamente para a inclusão reversa, para (ii) $(C \backslash A) \backslash(C \backslash B) \subseteq B \backslash A$ e analogamente para a reversa, etc. Durante a verificação das propriedades acima, fica claro que as propriedades (i), (iii) e (iv) valem também com " $\subseteq$ " no lugar de " $=* "$.) Além da questão prática e "computacional", a utilidade de propriedades como a acima reside na seguinte observação: em muitas situações a melhor maneira de provarmos que certo conjunto é finito (resp., infinito) será verificarmos que ele é "=*" a algum conjunto finito (resp., infinito). Os conjuntos finitos, por exemplo, são aqueles quase iguais ao conjunto vazio $\left([\emptyset]_{=^{*}}=[\omega]^{<\omega}\right)$. Observamos que em geral não valem as propriedades análogas às destacadas acima para a relação " $C^{*}$ "; por exemplo, se $P=\{2 k: k<\omega\}$ e $I=\{2 k+1: k<\omega\}$, então $P \subset^{*} \omega, I \subset^{*} \omega$ mas $P \cup I=\omega$. Vale porém que

(v) $A \subseteq^{*} B \Longleftrightarrow(\omega \backslash B) \subseteq^{*}(\omega \backslash A), \log \mathrm{o}$

(vi) $A \subset^{*} B \Longleftrightarrow(\omega \backslash B) \subset^{*}(\omega \backslash A)$.

Outro fato importante a respeito das pré-ordens $\left\langle\mathcal{P}(\omega), \subseteq^{*}\right\rangle$ e $\left\langle{ }^{\omega} \omega, \leqslant^{*}\right\rangle$ é o seguinte: as classes de equivalência por $=^{*}$ são sempre enumeráveis. Para ver isso, note que, se $F \subseteq \omega,[F]_{=*}=\left\{G \subseteq \omega: \exists A, B \in[\omega]^{<\omega}\right.$ tais que $\left.G=(F \cup A) \backslash B\right\}$. Então $\left|[F]_{=*}\right| \leqslant$ $\left|[\omega]^{<\omega} \times[\omega]^{<\omega}\right|=\omega$, e analogamente para $f \in{ }^{\omega} \omega$.

Dado um conjunto $X \neq \emptyset$, um filtro sobre $X$ é uma família $\mathcal{F} \subseteq \mathcal{P}(X)$ que satisfaz as condições $(i),($ ii $)$ e (iii) a seguir:

(i) $\emptyset \notin \mathcal{F}, X \in \mathcal{F}$

(ii) $(\forall A, B \in \mathcal{F})[A \cap B \in \mathcal{F}]$ 
(iii) $(\forall A \in \mathcal{F})\left(\forall B \subseteq X^{\prime}\right)[A \subseteq B \Rightarrow B \in \mathcal{F}]$

Um ultrafiltro sobre $X$ é um filtro sobre $X$ que é maximal no sentido da inclusão, isto é, $\mathcal{F}$ é um ultrafiltro sobre $X$ se $\mathcal{F}$ for um filtro sobre $X$ que não está contido propriamente em nenhum outro filtro sobre $X$. Sabe-se que:

(iv) Um filtro $\mathcal{F}$ sobre $X$ é um ultrafiltro se e só se

$$
(\forall A \subseteq X)[(A \in \mathcal{F}) \vee(X \backslash A \in \mathcal{F})]
$$

(v) Se $\mathcal{F} \subseteq \mathcal{P}(X)$ satisfaz a propriedade da intersecção finita (p.i.f.) - isto é, se $\left(\forall \mathcal{F}^{\prime} \in[\mathcal{F}]^{<\omega}\right)\left[\cap \mathcal{F}^{\prime} \neq \emptyset\right]$ - então existe algum ultrafiltro $\mathcal{U}$ sobre $X$ tal que $\mathcal{F} \subseteq \mathcal{U}$;

(vi) Se $\mathcal{U}$ é um ultrafiltro sobre $X$, então $\bigcap \mathcal{U}$ ou é vazia ou possui um único elemento.

Em se tratando de ultrafiltros, nosso principal interesse recairá no caso em que $X=\omega$. Seja $\mathcal{U}$ um ultrafiltro sobre $\omega ; \mathcal{U}$ será dito um ultrafiltro principal se existir $n<\omega$ para o qual $\mathcal{U}=\{A \subseteq \omega: n \in A\}$; caso contrário (i.e., se $\bigcap \mathcal{U}=\emptyset$ ), $\mathcal{U}$ será dito um ultrafiltro não-principal ou livre. Uma das principais propriedades dos ultrafiltros livres em $\omega$ é a seguinte:

(vii) Ultrafiltros livres em $\omega$ não possuem como elementos subconjuntos finitos de $\omega$.

Encerrando nossa introdução sobre Teoria dos Conjuntos, falemos um pouco a respeito de consistência e independência. Seja $S$ um conjunto de sentenças e $\varphi$ uma sentença. $\varphi$ é dita um teorema de $S$ se $\varphi$ é verdadeira em todos os modelos de $S$ (ou, equivalentemente, pelo Teorema da Completude, $\varphi$ é um teorema de $S$ se qualquer modelo de $S$ prova $\varphi$.). $\varphi$ é dita consistente com $S$ se existir algum modelo de $S$ no qual $\varphi$ seja verdadeira. $\varphi$ é dita independente de $S$ se tanto $\varphi$ como $\neg \varphi$ forem consistentes com $S$. Por exemplo, sabemos que o Axioma de Escolha e a Hipótese do Contínuo são independentes 
de $Z F$ (Gödel provou que $A C$ e $C H$ são consistentes com $Z F$, Cohen provou que a negação de ambas é consistente com $Z F$ ).

Neste trabalho, "Teorema" significa "teorema de ZFC". Quando alguma hipótese ou axioma adicional for utilizado, tal fato será destacado. Muitas afirmações sobre os chamados pequenos cardinais (que serão os principais objetos de estudo deste trabalho) são independentes de $Z F C$. Atualmente, a principal técnica para a obtenção de provas de consistência e de independência é o forcing, introduzido por Paul Cohen nos anos 60 na sua prova da consistência de $\neg A C$ e de $\neg C H$. Em nosso texto, não utilizaremos a técnica de forcing: privilegiaremos os resultados válidos em $Z F C$ sobre os nossos objetos de estudo e suas aplicações à topologia, sempre ressaltando, quando conveniente ou necessário, quais são os resultados de consistência ou de independência que estejam relacionados com o contexto em questão e que estejam disponíveis em nossa literatura, e em alguns casos dando outlines da demonstração desses resultados. Voltaremos a tratar de consistência e de independência no final do próximo capítulo.

\subsection{Topologia Geral}

Nossa principal referência em Topologia Geral é o livro de Engelking ([Eng89]). Porém, em muitas situações tal livro assume hipóteses de separação na definição de alguns conceitos, o que em geral não faremos; nesse sentido, adotaremos a linha do grupo de pesquisa em Topologia Geral e Conjuntística do IME/USP, que edita regularmente notas de aula para os cursos de graduação e pós-graduação do Instituto ([TJ97]). Para alguns resultados sobre espaços completamente metrizáveis (principalmente sobre o espaço dos irracionais), utilizamos o livro de Willard ([Wil70]).

Assumiremos conhecidos os conceitos básicos de topologia geral (topologia sobre um conjunto, abertos e fechados, interior e fecho, vizinhanças, funções (contínuas, abertas, fechadas, homeomorfismos), pontos de acumulação, base do espaço e base num ponto.). Em particular, um espaço topológico é um par $\langle X, \tau\rangle$, onde $X$ é o conjunto-suporte e $\tau$ é uma topologia sobre $X$. Quando não houver dúvidas sobre qual é a topologia dada ao conjunto-suporte, nos referiremos ao espaço topológico simplesmente como "espaço topológico $X$ " ou "espaço $X$ ". 
Axiomas de enumerabilidade e de separação: dizemos que um espaço $X$ satisfaz o primeiro axioma da enumerabilidade (ou é "primeiro enumerável", de "first countable") se todo ponto do espaço possui um sistema fundamental de vizinhanças que é enumerável; $X$ satisfaz o segundo axioma da enumerabilidade se possui uma base de abertos que é enumerável e $X$ satisfaz o terceiro axioma da enumerabilidade ( e é dito separável) se $X$ possui um subconjunto enumerável denso. Quanto aos axiomas de separação, assumiremos conhecidas as definições de espaço $T_{i}$ para $i=0,1,2,3,3 \frac{1}{2}$ e 4 ( $§ 1.5$ de [Eng]) e usaremos também os termos Hausdorff para $T_{2}$, regular para $T_{3}$, completamente regular para $T_{3 \frac{1}{2}} \mathrm{e}$ normal para $T_{4}$; os espaços $T_{i}$ são assumidos $T_{1}$ para $i \geqslant 3$.

Se $\langle X, \tau\rangle$ é um espaço topológico e $A \subseteq X$, a topologia de subespaço induzida por $\tau$ é $\tau^{\prime}=\tau\lceil A=\{A \cap O: O \in \tau\}$. Uma propriedade topológica (i.e., preservada por homeomorfismos) é dita hereditária se é preservada por "tomar subespaços", isto é, se um espaço $X$ satisfaz $P$ e $A$ é subespaço de $X$ então $A$ satisfaz $P$.

Se $\left\{X_{i}: i \in I\right\}$ é uma família de espaços topológicos, a topologia dada ao produto cartesiano $\prod_{i \in I} X_{i}$ é a chamada topologia-produto de Tychonoff, gerada pela base $\left\{\prod_{i \in I} V_{i}: V_{i}\right.$ é aberto em $X_{i}$ para todo $i$ e $\left.\left|\left\{i \in I: V_{i} \neq X_{i}\right\}\right|<\omega\right\}$. Uma propriedade $\stackrel{i \in I}{P}$ é dita produtiva se é preservada por "efetuar produtos", isto é, se $\left\{X_{i}: i \in I\right\}$ é tal que cada $X_{i}$ satisfaz $P$, então $\prod_{i \in I} X_{i}$ satisfaz $P$.

Se $X$ é um espaço topológico e $A \subseteq X$ é um subconjunto que é tanto um aberto como um fechado de $X$, dizemos que $A$ é um aberto-fechado ou clopen (por "closed and open"). Um espaço $X$ é dito zero-dimensional se possuir uma base formada por abertos-fechados. Por exemplo, um ordinal $\delta$ com a topologia da ordem é um espaço zerodimensional, pois a família dos intervalos \{]$\alpha, \beta]: \alpha<\beta<\delta\} \cup\{[0, \beta]: \beta<\delta\}$ é uma base de abertos-fechados para $\delta$. Os exemplos de espaços topológicos que efetivamente construiremos no último capítulo deste trabalho são todos zero-dimensionais.

Sempre consideraremos ordinais com a topologia da ordem; em particular, para $\kappa \leqslant \omega, \kappa$ é o espaço discreto com $\kappa$ pontos.

Um espaço $X$ é dito coletivamente Hausdorff se para todo $D \subseteq X$ fechado e discreto existe uma família de abertos disjuntos $\left\{U_{x}: x \in D\right\}$ tal que $\left.(\forall x \in D)\left[x \in U_{x}\right]\right)$.

Dois subconjuntos $A$ e $B$ de um espaço $X$ são ditos completamente separados se existe uma função contínua $f: X \mapsto[0,1]$ tal que $f(x)=0$ para $x \in A$ e $f(x)=1$ para $x \in B$. 
Se $X$ é um espaço e $A \subseteq X$, o derivado de $A$ (denotado por $A^{\prime}$ ) é o conjunto de todos os pontos de acumulação de $A$.

Se $X$ é um espaço e $A \subseteq X$ é tal que o interior seu fecho é vazio (i.e., $\operatorname{int}(\bar{A})=\emptyset$ ), dizemos que $A$ é um conjunto raro (em inglês, esses conjuntos são normalmente chamados de "nowhere dense"). Um subconjunto de $X$ é dito magro se for reunião enumerável de raros. Um espaço $X$ é dito um espaço de Baire se todo conjunto magro em $X$ tem interior vazio em $X$. Por exemplo, espaços completamente metrizáveis são espaços de Baire.

Uma $\pi$-base de um espaço topológico $X$ é uma família de abertos não-vazios $\mathcal{V}$ tal que, para todo aberto não-vazio $U$ de $X$, existe $V \in \mathcal{V}$ tal que $V \subseteq U$.

Seja $X$ um espaço, $A \subseteq X$ e $x \in X ; x$ é dito um ponto de acumulação completo de $A$ se para toda vizinhança aberta $U$ de $x$ vale que $|U \cap A|=|A|$.

Suporemos conhecidas as definições de espaço compacto, espaço enumeravelmente compacto e espaço de Lindelöf, enunciadas a partir dos conceitos de coberturas e subcoberturas abertas (e não assumiremos aqui que um espaço compacto é Hausdorff ou $T_{1}$ ). Destacaremos agora algumas equivalências que nos serão muitíssimo úteis.

Para a compacidade, temos que são equivalentes:

(i) $X$ é compacto.

(ii) Toda família de fechados com a p.i.f. em $X$ possui intersecção não-vazia.

(iii) Todo subconjunto infinito de $X$ possui um ponto de acumulação completo.

Para a compacidade enumerável, temos as seguintes equivalências:

(i) $X$ é enumeravelmente compacto.

(ii) Toda família enumerável de fechados com a p.i.f. em $X$ possui intersecção não-vazia.

(iii) Todo subconjunto infinito e enumerável de $X$ possui um ponto de acumulação completo.

A compacidade e a compacidade enumerável são propriedades hereditárias para subespaços fechados. Disto - ou ainda das caracterizações acima - conclui-se que espaços compactos e espaços enumeravelmente compactos não possuem subconjuntos infinitos que sejam fechados e discretos. 
Seja $X$ um espaço topológico, $x \in X$ e $A \subseteq X$ tal que $|A|=\omega$. Dizemos que $A$ converge para $x$ se para qualquer vizinhança aberta $V$ de $x$ tem-se $A \backslash V$ finito, isto é, $A$ converge para $x$ se qualquer vizinhança aberta de $x$ possui todos a menos de no máximo finitos elementos de $A$.

Um espaço $X$ é dito seqüencialmente compacto se todo subconjunto infinito e enumerável de $X$ possui um subconjunto infinito que converge para algum ponto.

Um espaço $X$ é dito subseqüencial se para todo $A \subseteq X$ infinito e enumerável e para todo $x$ ponto de acumulação completo de $A$ existe $N \subseteq A$ infinito e que converge para $x$.

Seja $f: \omega \mapsto X$ uma seqüência num espaço $X . x \in X$ é dito ponto de acumulação de $f$ se, para toda vizinhança aberta $V$ de $x,|\{n<\omega: f(n) \in V\}|=\left|f^{-1}[V]\right|=\omega . \quad f$ converge para um elemento $x \in X$ se, para toda vizinhança aberta $V$ de $x, \mid\{n<\omega$ : $f(n) \notin V\} \mid<\omega$. Em espaços de Hausdorff, os limites de uma sequência são únicos.

Um subconjunto $A$ de um espaço $X$ é dito seqüencialmente fechado se $A$ for fechado para limites de seqüências, isto é, se $\left\langle x_{n}: n<\omega\right\rangle$ é uma sequência de elementos de $A$ que converge para um ponto $x \in X$, então $x \in A$. Todo fechado é seqüencialmente fechado, mas a recíproca não é verdadeira (considere, por exemplo, $X=\omega_{1}+1$ com a topologia da ordem; $\omega_{1}$ é um subespaço seqüencialmente fechado de $X$ que não é fechado em $X$.).

Um espaço $X$ é dito seqüencial se todo subconjunto seqüencialmente fechado de $X$ é um fechado de $X$. Equivalentemente, $X$ é seqüencialmente fechado se, para todo $A \subseteq X$ não-fechado, existe uma sequiência de pontos de $A$ que converge para um ponto de $X \backslash A$.

Temos então os seguintes fatos:

(i) Espaços compactos são enumeravelmente compactos.

(ii) Espaços seqüencialmente compactos são enumeravelmente compactos.

(iii) Espaços enumeravelmente compactos e subseqüenciais são seqüencialmente compactos.

(iv) As propriedades "ser enumeravelmente compacto" e "ser seqüencialmente compacto" são equivalentes na classe dos espaços seqüenciais ([Eng89], 3.10.31) 
Exemplo 1.1 Um ordinal $\delta$ de cofinalidade não-enumerável é seqüencialmente compacto (portanto, enumeravelmente compacto), não-compacto e subseqüencial. $\delta$ é seqüencial se e só se $\delta=\omega_{1}$.

Prova: Seja $\delta$ um ordinal limite $\operatorname{com} c f(\delta)>\omega$. $\delta$ não é compacto pois a cobertura aberta $\{[0, \alpha[: \alpha<\delta\}$ não possui subcobertura finita (obviamente, isso valeria mesmo que $\delta$ fosse limite com cofinalidade enumerável) . Para a compacidade seqüencial, tomamos $A \subset \delta$ $\operatorname{com}|A|=\omega, \zeta$ o tipo de ordem de $\langle A, \in\rangle$ e $\left\{a_{\xi}: \xi<\zeta\right\}$ a enumeração crescente (canônica) de $A$. Claramente, $\omega \leqslant \zeta<\omega_{1}$. Considere então o ordinal $\beta=\sup \left\{a_{n}: n<\omega\right\}$; é imediato que $\beta$ é ponto de acumulação completa de $A$ e que $\left\{a_{n}: n<\omega\right\}$ converge para $\beta$. $\delta$ é subseqüencial pois os pontos de acumulação de subconjuntos enumeráveis e infinitos de $\delta$ são ordinais limite e portanto limites de enumerações crescentes. Se $\delta=\omega_{1}$, $\delta$ é seqüencial pois é primeiro enumerável e espaços primeiro enumeráveis são seqüenciais ([Eng89], 1.6.14). Se $\delta>\omega_{1}, \omega_{1}$ é um subespaço seqüencialmente fechado de $\delta$ que não é fechado.

Um espaço $X$ é dito $\omega$-limitado se todo subconjunto enumerável e infinito de $X$ está contido em algum subespaço compacto de $X$. No caso de $X$ ser $T_{2}$, isso é equivalente a dizer que todo subconjunto enumerável e infinito de $X$ possui fecho compacto.

Um espaço $X$ é dito $\sigma$-compacto se $X$ pode ser escrito como uma reunião enumerável de subespaços compactos.

Um espaço $X$ é dito localmente compacto se todo ponto $x \in X$ possui um sistema fundamental de vizinhanças compactas. Para espaços $T_{2}$, isso é equivalente a dizer que para cada $x \in X$ e para cada $W$ vizinhança aberta de $X$ existe uma vizinhança aberta $V$ de $x$ tal que $\bar{V}$ é um subconjunto compacto de $W$.

Se $X$ é um espaço e $\mathcal{U}$ é uma cobertura de $X$, dizemos que uma cobertura $\mathcal{V}$ de $X$ é um refinamento de $\mathcal{U}$ se para todo $V \in \mathcal{V}$ existe $U \in \mathcal{U}$ tal que $V \subseteq U$. $\mathcal{V}$ é dito um refinamento aberto de $\mathcal{U}$ se todos os elementos de $\mathcal{V}$ são abertos de $X$. Um espaço $X$ é dito paracompacto se toda cobertura aberta de $X$ possui um refinamento aberto localmente finito, e um espaço $X$ é dito enumeravelmente paracompacto se toda cobertura aberta enumerável de $X$ possui um refinamento aberto localmente finito.

Se $A$ é um conjunto e $X$ é um espaço, ${ }^{A} X$ será sempre considerado com a topologiaproduto de Tychonoff. Em algumas situações, esse espaço produto de suporte ${ }^{A} X$ será 
denotado por $X^{A}$.

$\mathbb{R}$ denota a "reta real" com sua topologia usual. $\mathbb{Q}$ e $\mathbb{P}$ denotam, respectivamente, o espaço dos racionais e dos irracionais com a topologia de subespaço da reta. Uma importante propriedade do espaço $\mathbb{P}$ dos irracionais é que $\mathbb{P}$ é homeomorfo ao espaçoproduto $\omega^{\omega}$ (ver exercício $24 \mathrm{~K}$ de [Wil70]).

Um dos principais espaços estudados em Topologia Conjuntística é o espaço $\beta \omega$, o compactificado de Stone-Čech do espaço discreto $\omega$. O artigo [Rud56] de W.Rudin é uma excelente introdução aos aspectos mais elementares de $\beta \omega$ e está escrito numa linguagem bastante acessível. Destacaremos aqui os seguintes fatos básicos a respeito de $\beta \omega$ :

(i) $\beta \omega$ é (homeomorfo a)o espaço topológico cujo suporte é o conjunto de todos os ultrafiltros sobre $\omega$ e cuja topologia é dada pela base $\{V(E): E \subseteq \omega\}$, onde para cada $E \subseteq \omega$ o aberto básico $V(E)$ é dado por $V(E)=\{\Omega: \Omega$ é ultrafiltro sobre $\omega$ e $E \in \Omega\}$. O subespaço denso de $\beta \omega$ que é homeomorfo a $\omega$ é o conjunto dos ultrafiltros principais, sendo que cada natural $n$ está associado ao ultrafiltro principal $\Omega_{n}=\{A \subseteq \omega: n \in A\}$, $\operatorname{assim} \omega \equiv\left\{\Omega_{n}: n<\omega\right\}$. O homeomorfismo é imediato, pois, como $V(\{n\})=\left\{\Omega_{n}\right\}$ para todo natural $n,\left\{\Omega_{n}: n<\omega\right\}$ é um subespaço discreto enumerável de $\beta \omega$. Em resumo, $\omega$ é discreto e denso em $\beta \omega$ - e vale também que $\{\{n\}: n<\omega\}$ é uma $\pi$-base de $\beta \omega$.

(ii) A base $\{V(E): E \subseteq \omega\}$ é tal que todos os seus abertos são também fechados - basta notar que $V(\omega \backslash E)=\beta \omega \backslash V(E)$ para todo $E \subseteq \omega$ - logo $\beta \omega$ é zero-dimensional.

(iii) Do fato de que os pontos de $\omega$ são isolados vem que $\beta \omega \backslash \omega=\omega^{*}$ - que é o conjunto dos ultrafiltros livres sobre $\omega$ - é um subespaço fechado de $\beta \omega$ e portanto compacto. Como ultrafiltros livres não possuem como elementos subconjuntos finitos de $\omega$, para todo $E \in[\omega]^{<\omega}$ tem-se $\omega^{*} \cap V(E)=\emptyset$. Assim, a topologia de $\omega^{*}$ é dada pela base $\left\{W(E): E \in[\omega]^{\omega}\right\}$ onde para cada $E \in[\omega]^{\omega}$ o aberto básico $W(E)$ é definido por $W(E)=\{\Omega: \Omega$ é ultrafiltro livre sobre $\omega$ e $E \in \Omega\}$. Cada um dos $W(E)$ é fechado em $\omega^{*}$, portanto $\omega^{*}$ também é zero-dimensional.

(iv) Dados $E_{1}, E_{2} \in[\omega]^{\omega}$ quaisquer, vale que $W\left(E_{1}\right) \subseteq W\left(E_{2}\right) \Leftrightarrow E_{1} \subseteq^{*} E_{2}$ e $W\left(E_{1}\right) \subset$ 
$W\left(E_{2}\right) \Leftrightarrow E_{1} \subset^{*} E_{2}$, isto é, $W\left(E_{1}\right)=W\left(E_{2}\right) \Leftrightarrow E_{1}={ }^{*} E_{2}$.

$(v)$ Todo aberto-fechado de $\omega^{*}$ é da forma $W(E)$ para algum $E \in[\omega]^{\omega}$.

(vi) A intersecção de qualquer família enumerável de abertos de $\omega^{*}$ ou é vazia ou contém um aberto não-vazio.

(vii) $|\beta \omega|=2^{\mathfrak{c}}$ e $\beta \omega$ omite cardinais menores que $2^{\mathfrak{c}}$, isto é, todo subconjunto fechado e infinito de $\beta \omega$ tem cardinalidade $2^{\mathfrak{c}}$. Isso ocorre porque, se $F \subseteq \beta \omega$ é fechado e infinito, então $F$ possui um subespaço que é homeomorfo a $\beta \omega$ ([Eng89], 3.6.14). Segue que as únicas seqüências convergentes em $\beta \omega$ são as quase-constantes e que $\beta \omega$ não é seqüencialmente compacto.

(viii) Sobre os elementos de $\beta \omega$ está definida uma pré-ordem denominada pré-ordem de $R u d i n$-Keisler, denotada por $\leqslant_{R K}$ e definida da seguinte forma: para quaisquer $u, v \in \beta \omega$, $u \leqslant_{R K} v \Longleftrightarrow$ existe $f \in{ }^{\omega} \omega$ tal que $u=\left\{A \subseteq \omega: f^{-1}[A] \in v\right\}=\bar{f}(v)$

Em trabalhos como este, em que a Topologia e a Teoria dos Conjuntos convivem, freqüentemente as chamadas funções cardinais desempenham um importante papel. Uma função cardinal é uma "função" (função-classe) $\varphi$, definida na classe dos espaços topológicos com valores na classe dos cardinais infinitos, e que é preservada por homeomorfismos, i.e., se $X$ e $Y$ são espaços homeomorfos e $\varphi$ é uma função cardinal então $\varphi(X)=\varphi(Y)$. Funções cardinais generalizam para maiores cardinalidades idéias como as que definem os três axiomas de enumerabilidade ("ser primeiro enumerável", "possuir base enumerável", "ser separável"), dentre muitas outras propriedades. Nossa principal referência para funções cardinais é o artigo de Hodel ([Hod84]). Não são muitas as funções cardinais que aparecem em nosso trabalho; além da cardinalidade do espaço, que para espaços infinitos é simplesmente a cardinalidade do conjunto-suporte, as funções cardinais às quais nos referiremos são basicamente as seguintes: 
- peso de X:

$w(X)=\min \{|\mathcal{B}|: \mathcal{B}$ é uma base para $X\}+\omega$

- caráter de $X$ :

$\chi(X)=\sup \{\chi(p, X): p \in X\}+\omega$

onde $\chi(p, X)$ é o caráter de $p$ em $X$ :

$\chi(p, X)=\min \{|\mathcal{B}|: \mathcal{B}$ é uma base local para $p$ em $X\}$

- tightness de $X$ :

$t(X)=\sup \{t(p, X): p \in X\}+\omega$

onde $t(p, X)$ é o tightness de $p$ em $X$ :

$$
\begin{gathered}
t(p, X)=\min \{\kappa: \text { para todo } Y \subseteq X \text { para o qual } p \in \bar{Y}, \text { existe } A \subseteq Y \text { com } \\
|A| \leqslant \kappa \text { e tal que } p \in \bar{A}\}
\end{gathered}
$$

Finalmente, um comentário de cunho bibliográfico: durante a elaboração deste trabalho, duas obras - coletâneas de artigos - foram verdadeiros "livros de cabeceira": o Handbook of Set Theoretic Topology, editado por Kenneth Kunen e Jerry Vaughan em 1984, e o Open Problems in Topology, editado por Jan Van Mill e George M.Reed em 1990. Devido à constante presença de citações de artigos publicados nessas duas obras, freqüentemente nos referiremos a elas simplesmente como, respectivamente, Handbook e Open Problems. 


\section{Capítulo 2}

\section{Seis pequenos cardinais}

\subsection{Apresentação dos seis pequenos cardinais}

Elementos de ${ }^{\omega} \omega$ e de $[\omega]^{\omega}$ são exemplos de conjuntos enumeráveis infinitos; uma função $f$ de $\omega$ em $\omega$ possui $\aleph_{0}$ pares ordenados e um subconjunto infinito $X \subseteq \omega$ possui $\aleph_{0}$ números naturais. É razoável que, para conjuntos de tal cardinalidade, conjuntos finitos sejam "desprezíveis". Assim, se $A, B \in[\omega]^{\omega}$, pode-se dizer que $A$ está "essencialmente contido" em $B$ se apenas finitos elementos de $A$ não sejam também elementos de $B$; analogamente, dadas $f, g \in{ }^{\omega} \omega$, pode-se dizer que $f$ é "essencialmente menor ou igual" a $g$ se o conjunto dos pontos $n$ nos quais $g(n)$ é menor que $f(n)$ seja finito. Observamos então que as definições dadas no capítulo anterior para as pré-ordens $\left\langle\mathcal{P}(\omega), \subseteq^{*}\right\rangle$ e $\left\langle{ }^{\omega} \omega, \leqslant^{*}\right\rangle$ formalizam essa idéia intuitiva de que "conjuntos finitos podem ser desprezados". Essa idéia intuitiva é um ponto comum às definições dos seis cardinais com os quais trabalharemos; veremos agora diferentes maneiras sob as quais essa idéia se manifesta.

Dois conjuntos $A, B \in[\omega]^{\omega}$ são ditos a.d. ("almost disjoint") se $|A \cap B|<\omega$. Uma família $\mathcal{F} \subseteq[\omega]^{\omega}$ é dita uma família a.d. em $\omega$ se para quaisquer $A, B \in \mathcal{F}$ distintos temse $A$ e $B$ a.d. Finalmente, uma subfamília $\mathcal{A}$ de $[\omega]^{\omega}$ é dita uma família m.a.d.(maximal almost disjoint) em $\omega$ se $\mathcal{A}$ for uma família a.d. que é maximal para essa propriedade, no sentido da inclusão. Observamos que $\{\omega\}$ é uma família m.a.d. finita; aliás, qualquer partição finita de $\omega$ formada por subconjuntos infinitos e disjuntos será uma família m.a.d. finita. Logo, as famílias m.a.d. nas quais reside algum interesse são as famílias m.a.d. 
infinitas. Definimos então o pequeno cardinal $\mathfrak{a}$ da seguinte forma:

$\mathfrak{a}=\min \left\{|\mathcal{A}|: \mathcal{A} \subseteq[\omega]^{\omega}\right.$ é uma família m.a.d. infinita em $\left.\omega\right\}$

Tratemos agora da pré-ordem $\left\langle{ }^{\omega} \omega, \leqslant^{*}\right\rangle$. Definimos os conceitos de famílias ilimitadas e dominantes para pré-ordens quaisquer, no capítulo anterior, mas não custa lembrar: $B \subseteq{ }^{\omega} \omega$ é dita uma família ilimitada se for ilimitada em $\left\langle{ }^{\omega} \omega, \leqslant^{*}\right\rangle$ (i.e., se $(\forall f \in$ $\left.\left.{ }^{\omega} \omega\right)(\exists g \in B)\left[g \mathbb{K}^{*} f\right]\right)$ e $D \subseteq{ }^{\omega} \omega$ é dita uma família dominante se for cofinal em $\left\langle{ }^{\omega} \omega, \leqslant^{*}\right\rangle$ (i.e., se $\left.\left(\forall f \in{ }^{\omega} \omega\right)(\exists g \in D)\left[f \leqslant{ }^{*} g\right]\right)$. Observamos aqui que $\left\langle{ }^{\omega} \omega, \leqslant^{*}\right\rangle$ não possui elementos maximais, pois dada $f \in{ }^{\omega} \omega$ a funçào $g=f+1$ definida por $g(n)=f(n)+1$ para todo $n<\omega$ satisfaz $f<{ }^{*} g$.

Uma subfamília $\mathcal{E} \subseteq[\omega]^{\omega}$ é dita uma escala em $\left\langle{ }^{\omega} \omega, \leqslant^{*}\right\rangle$ se for dominante e bemordenada por $\subseteq^{*}$. Famílias dominantes são claramente ilimitadas, e é fácil ver que famílias finitas não podem ser ilimitadas. Assim, definimos e são infinitos os seguintes pequenos cardinais:

$$
\begin{aligned}
& \mathfrak{b}=\min \left\{|B|: B \subseteq{ }^{\omega} \omega \text { é uma família ilimitada em }\left\langle{ }^{\omega} \omega, \leqslant^{*}\right\rangle\right\} \\
& \mathfrak{d}=\min \left\{|D|: D \subseteq{ }^{\omega} \omega \text { é uma família dominante em }\left\langle{ }^{\omega} \omega, \leqslant^{*}\right\rangle\right\}
\end{aligned}
$$

Os próximos cardinais serão definidos a partir de subfamílias de $[\omega]^{\omega}$ que satisfaçam condições relacionadas à pré-ordem $\left\langle\mathcal{P}(\omega), \subseteq^{*}\right\rangle$. Vamos introduzir algumas terminologias. Se $\mathcal{F} \subseteq[\omega]^{\omega}$, dizemos que um conjunto $A$ é uma pseudo-interseç̧ão de $\mathcal{F}$ se $(\forall F \in \mathcal{F})\left[A \subseteq^{*} F\right]$ e dizemos que $\mathcal{F}$ satisfaz a s.f.i.p. (strong finite intersection property - propriedade forte da intersecção finita) se toda sua subfamília finita e não vazia possui intersecção infinita. Se $\mathcal{F}$ possui uma pseudo-intersecção infinita, $\mathcal{F}$ satisfaz a s.f.i.p., mas a recíproca não é verdadeira; veremos em breve que qualquer ultrafiltro não-principal em $\omega$ é um contra-exemplo. $\mathcal{T} \subseteq[\omega]^{\omega}$ é dita uma torre se $\mathcal{T}$ é bem-ordenada por $\supset^{*}$ e não possui pseudo-intersecção infinita. É fácil ver que torres possuem a s.f.i.p. (basta notar que, se $A_{1}, A_{2}, \ldots, A_{n} \in[\omega]^{\omega}$ são tais que $A_{1} \subseteq^{*} A_{2} \subseteq^{*} \ldots \subseteq^{*} A_{n}$, então $\left.A_{1}=^{*} \bigcap_{i=1}^{n} A_{i}\right)$. Também é imediato que famílias finitas (não-vazias) de conjuntos infinitos que satisfaçam a s.f.i.p. possuem pseudo-intersecções infinitas (para o caso de famílias vazias, veremos que tal condição vale por vacuidade). Assim, definimos e são infinitos os seguintes pequenos 
cardinais:

$$
\begin{aligned}
& \mathfrak{p}=\min \left\{|\mathcal{F}|: \mathcal{F} \subseteq[\omega]^{\omega}\right. \text { satisfaz a s.f.i.p. mas não possui pseudo-intersecção } \\
& \text { infinita }\} \\
& \mathfrak{t}=\min \left\{|\mathcal{T}|: \mathcal{T} \subseteq[\omega]^{\omega} \text { é uma torre }\right\} \\
& \text { Finalmente, } \mathcal{S} \subseteq[\omega]^{\omega} \text { é dita uma família splitting ("cisante") se } \\
& \qquad \neg\left(\exists A \in[\omega]^{\omega}\right)(\forall S \in \mathcal{S})\left[\left(A \subseteq^{*} S\right) \vee\left(A \subseteq^{*}(\omega \backslash S)\right)\right]
\end{aligned}
$$

ou, equivalentemente, $\mathcal{S}$ é splitting se $\left(\forall A \in[\omega]^{\omega}\right)(\exists S \in \mathcal{S})[|A \cap S|=|A \backslash S|=\omega]$. Famílias finitas não podem ser splitting pois, se $\mathcal{S}$ é família splitting e $S \in \mathcal{S}$ então $\mathcal{S} \backslash\{S\}$ é splitting. Então é infinito o pequeno cardinal $\mathfrak{s}$ dado por

$$
\mathfrak{s}=\min \left\{|\mathcal{S}|: \mathcal{S} \subseteq[\omega]^{\omega} \text { é uma família splitting }\right\}
$$

Com o intuito de facilitar posteriores consultas, repetiremos as definições de nossos seis cardinais:

Definição 2.1 Os pequenos cardinais $\mathfrak{a}, \mathfrak{b}, \mathfrak{d}, \mathfrak{p}, \mathfrak{s}$ e $\mathfrak{t}$ são dados por:

$$
\begin{aligned}
& \mathfrak{a}=\min \left\{|\mathcal{A}|: \mathcal{A} \subseteq[\omega]^{\omega} \text { é uma família m.a.d. infinita em } \omega\right\} \\
& \mathfrak{b}=\min \left\{|B|: B \subseteq{ }^{\omega} \omega \text { é uma família ilimitada em }\left\langle{ }^{\omega} \omega, \leqslant^{*}\right\rangle\right\} \\
& \mathfrak{d}=\min \left\{|D|: D \subseteq{ }^{\omega} \omega \text { é uma família dominante em }\left\langle{ }^{\omega} \omega, \leqslant^{*}\right\rangle\right\} \\
& \mathfrak{p}=\min \left\{|\mathcal{F}|: \mathcal{F} \subseteq[\omega]^{\omega}\right. \text { satisfaz a s.f.i.p. mas não possui pseudo-intersecção } \\
& \qquad \text { infinita }\} \\
& \mathfrak{s}=\min \left\{|\mathcal{S}|: \mathcal{S} \subseteq[\omega]^{\omega} \text { é uma família splitting }\right\} \\
& \mathfrak{t}=\min \left\{|\mathcal{T}|: \mathcal{T} \subseteq[\omega]^{\omega} \text { é uma torre }\right\}
\end{aligned}
$$

Erick van Douwen e Jerry Vaughan foram os responsáveis pela notação mnemônica e pela escolha de letras germânicas para esses cardinais. A escolha das letras germânicas é 
uma influência do uso da letra germânica "c" para a cardinalidade do continuum. Em cada um dos casos, a letra foi escolhida a partir do conceito principal envolvido na definição do cardinal: assim, a é o "almost disjointness number", b é o "(un)bounding number", $\mathfrak{d}$ é o "Dominating number", s é o "splitting number" e $t$ é o "tower number". A letra p é derivada do conceito de $P$-pontos; no final da próxima seção, a relação entre o conceito de $P$-ponto (que ainda não definimos) e o cardinal $\mathfrak{p}$ ficará mais clara.

Veremos agora que, se $\mu$ é qualquer um de nossos seis cardinais, valerá então $\omega_{1} \leqslant \mu \leqslant \mathfrak{c}$. A limitação superior por c é evidente, já que $|\mathcal{P}(\omega)|=\left|{ }^{\omega} \omega\right|=\left|[\omega]^{\omega}\right|=\mathfrak{c}$. Seguem as verificações de que nossos cardinais estão bem definidos (isto é, que os conjuntos de cardinais dos quais eles são mínimos são não vazios) e que cada um deles é nãoenumerável. Observamos que uma verificação desse tipo é necessária num primeiro contato com esses cardinais, já que, até o momento, nada nos garante que exista uma torre, por exemplo.

$$
\omega_{1} \leqslant \mathfrak{a} \leqslant \mathfrak{c}
$$

Para garantir a existência de uma família m.a.d. infinita em $\omega$, tomamos uma família $\mathcal{A}$, infinita e a.d. e aplicamos o Lema de Zorn à ordem parcial $\langle A, \subseteq\rangle$, onde $A=\{\mathcal{F} \subseteq \mathcal{P}(\omega): \mathcal{A} \subseteq \mathcal{F}$ e $\mathcal{F}$ é a.d. $\}$. Note que podemos tomar como família $\mathcal{A}$ uma família efetivamente disjunta, por exemplo uma partição de $\omega$ em $\omega$ conjuntos infinitos e disjuntos.

O fato de uma família a.d. de tamanho $\kappa$ num cardinal regular $\kappa(\kappa \geqslant \omega)$ não poder ser maximal é bastante conhecido, e é o primeiro resultado do cap.2 do livro de Kunen ([Kun80]); apresentaremos uma demonstração para $\kappa=\omega$ por cortesia. Seja $\mathcal{A}=$ $\left\{A_{n}: n<\omega\right\}$ uma família a.d. em $\omega$. Definimos então uma família $\left\{B_{n}: n<\omega\right\}$ pondo $B_{0}=A_{0}$ e $B_{n+1}=A_{n+1} \backslash \bigcup_{m \leqslant n} A_{m}$. Observamos que cada $B_{n}$ é não vazio $\left(B_{0}=A_{0} \neq \emptyset \mathrm{e}\right.$ $B_{n+1}=A_{n+1} \backslash \bigcup_{m \leqslant n}\left(A_{n+1} \cap A_{m}\right)$, e $\left|A_{n+1} \cap A_{m}\right|<\omega$ se $\left.m \leqslant n\right)$ e que os $B_{n}$ são claramente disjuntos. Assim, defino $b_{n}=\min \left(B_{n}\right)$ e o conjunto $B=\left\{b_{n}: n<\omega\right\}$ é um subconjunto infinito de $\omega$. Tem-se também $B \cap A_{n} \subseteq\left\{b_{0}, \ldots, b_{n}\right\}$ para todo $n$ e assim $\mathcal{A} \cup\{B\}$ é uma família a.d. que contém propriamente a família $\mathcal{A}$, logo nenhuma família a.d. enumerável 
em $\omega$ pode ser maximal.

$$
\omega_{1} \leqslant \mathfrak{b}, \mathfrak{d} \leqslant \mathfrak{c}
$$

Já comentamos o fato de que $\left\langle{ }^{\omega} \omega, \leqslant^{*}\right\rangle$ não possui elementos maximais, logo ${ }^{\omega} \omega$ é ilimitado (e dominante!) em si mesmo, portanto $\mathfrak{b}$ e $\mathfrak{d}$ estão bem definidos.. Verifiquemos que uma subfamília enumerável e arbitrária de ${ }^{\omega} \omega$ não pode ser ilimitada. Tome $B=$ $\left\{f_{n}: n<\omega\right\}$ uma família arbitrária de funções de $\omega$ em $\omega$. Defina então $g: \omega \mapsto \omega$ pondo $g(n)=\max \left\{f_{i}(j): i, j \leqslant n\right\}+1$. Temos que, dado $m<\omega$ qualquer, $\{n<\omega: g(n) \leqslant$ $\left.f_{m}(n)\right\} \subseteq\{0,1, \ldots, m\}, \operatorname{logo}(\forall m<\omega)\left[f_{m} \leqslant{ }^{*} g\right]$ e g é limitante superior de $B$. Segue também que $\omega_{1} \leqslant \mathfrak{d}$, já que uma família enumerável não pode ser dominante pois nesse caso seria também ilimitada.

$$
\omega_{1} \leqslant \mathfrak{p} \leqslant \mathfrak{c}
$$

Um ultrafiltro não-principal qualquer $\mathcal{U}$ em $\omega$ é uma família com a s.f.i.p (obviamente) e que não possui pseudo-intersecção infinita. A não-existência de pseudointersecções infinitas é consequência imediata do fato de que, se $A \in[\omega]^{\omega}$ e $\mathcal{U}$ é um ultrafiltro, então $A \in \mathcal{U}$ ou $\omega \backslash A \in \mathcal{U}$ e é fácil mostrar nessas condições que $A$ não é pseudo-intersecção infinita de $\mathcal{U}$ (se $\omega \backslash A \in \mathcal{U}$, não há o que fazer, e se $A \in \mathcal{U}$ uma decomposição de $A$ na forma $A=A_{1} \cup A_{2} \operatorname{com} A_{1}, A_{2}$ infinitos e disjuntos resolve a questão.).

A subfamília vazia de $[\omega]^{\omega}$ satisfaz a s.f.i.p. por vacuidade, mas, também por vacuidade, $\omega$ é uma pseudo-intersecção infinita da família vazia; daí, p $>0$. Mostraremos agora que, se $\mathcal{F} \subseteq[\omega]^{\omega}$ é uma família arbitrária enumerável com a s.f.i.p., então $\mathcal{F}$ possui uma pseudo-intersecção infinita $A$. De fato: para uma tal $\mathcal{F}=\left\{F_{n}: n<\omega\right\}$, definimos recursivamente $a: \omega \mapsto \omega$ pondo $a(0)=a_{0}=\min \left(F_{0}\right)$ e $a(n+1)=a_{n+1}=$ $\min \left(\left(\bigcap_{k \leqslant n+1} F_{k}\right) \backslash\left\{a_{0}, \ldots, a_{n}\right\}\right)$ para cada $n<\omega$. Segue que $A=\left\{a_{n}: n<\omega\right\}=i m(a)$ é uma pseudo-intersecção infinita de $\mathcal{F}$, pois $A \backslash F_{0}=\emptyset$ e para $0<m<\omega$ tem-se $A \backslash F_{m} \subseteq\left\{a_{0}, \ldots, a_{m-1}\right\}$.

(4) $\omega_{1} \leqslant \mathfrak{s} \leqslant \mathfrak{c}$ 
A existência de famílias splitting é imediata: por exemplo, a cada $A \in[\omega]^{\omega}$, fixamos $S_{A} \in[\omega]^{\omega}$ tal que $\left|A \cap S_{A}\right|=\left|A \backslash S_{A}\right|=\omega$. A família $\mathcal{S}=\left\{S_{A}: A \in[\omega]^{\omega}\right\}$ é claramente splitting.

Para mostrarmos que uma família enumerável $\mathcal{S}=\left\{S_{n}: n<\omega\right\} \subseteq[\omega]^{\omega}$ não pode ser splitting, lançamos mão de um argumento topológico por excelência. Definimos $\left\{f_{n}: n<\omega\right\} \subseteq{ }^{\omega} 2$ pondo, para $n, m<\omega$ arbitrários,

$$
f_{n}(m)= \begin{cases}1 & \text { se } n \in S_{m} \\ 0 & \text { caso contrário }\end{cases}
$$

Como ${ }^{\omega_{2}} 2$ é seqüencialmente compacto (pois é um produto enumerável de espaços seqüencialmente compactos ([Eng89], 3.10.35)), podemos tomar uma subsequência convergente $\left\langle f_{n_{k}}: k<\omega\right\rangle$. Seja $A=\left\{n_{k}: k<\omega\right\}$. Afirmamos que $(\forall n<\omega)\left[\left(\left|A \cap S_{n}\right|<\right.\right.$ $\left.\omega) \vee\left(\left|A \backslash S_{n}\right|<\omega\right)\right]$. De fato: seja $m<\omega$ fixado. Como $\left\langle f_{n_{k}}: k<\omega\right\rangle$ é convergente em ${ }^{\omega} 2$, temos $\left\langle f_{n_{k}}(m): k<\omega\right\rangle$ convergente em $2=\{0,1\}$ (com a topologia discreta). Se $\left\langle f_{n_{k}}(m): k<\omega\right\rangle$ convergir para 1, então apenas um número finito de elementos de $A$ não pertence a $S_{m}$, donde segue $\left|A \backslash S_{m}\right|<\omega$, e, com o resultado análogo para o caso em que a sequência converge para zero, A é um conjunto infinito que não pode ser cindido por nenhum elemento de $\mathcal{S}$, e portanto $\mathcal{S}$ não é splitting.

$$
\omega_{1} \leqslant \mathfrak{t} \leqslant \mathfrak{c}
$$

Para garantirmos a existência de torres, fixaremos uma classe de representantes $S$ da relação de equivalência $=^{*}$ - o que equivale a trabalhar no quociente de $[\omega]^{\omega}$ por $=^{*}$ - e construiremos uma torre com os elementos de $S$ (ou seja, estamos automaticamente desprezando argumentos repetitivos sobre a não importância de "diferenças finitas" entre os conjuntos tomados). Observamos que, em $S, \supseteq^{*}$ é uma ordem parcial, pois está garantida a antissimetria. Aplicamos agora o Lema de Zorn à ordem parcial $\langle\mathcal{C}, \triangleleft\rangle$, onde $\mathcal{C}=\left\{\left\langle\mathcal{A}, \supseteq_{\mathcal{A}}^{*}\right\rangle: \mathcal{A} \subseteq S\right.$ e $\supseteq_{\mathcal{A}}^{*}$ bem-ordena $\left.\mathcal{A}\right\}$, e

$$
\left\langle\mathcal{A}, \supseteq_{\mathcal{A}}^{*}\right\rangle \triangleleft\left\langle\mathcal{B}, \supseteq_{\mathcal{B}}^{*}\right\rangle \Longleftrightarrow \mathcal{A} \subseteq \mathcal{B}, \supseteq_{\mathcal{A}}^{*} \subseteq \supseteq_{\mathcal{B}}^{*} \text { e }(\forall F \in \mathcal{A})(\forall G \in \mathcal{B} \backslash \mathcal{A})\left[F \supseteq^{*} G\right]
$$

(Note que estamos exigindo que a ordem parcial $\triangleleft$ caracterize o que se costuma denominar "end-extension": uma subfamília $\mathcal{A}$ de $S$, com a ordem induzida por $\supseteq^{*}, \triangleleft$ precede uma subfamília $\mathcal{B}$ se estiver contida nesta última e se a ordem induzida nesta 
última estender "por cima" a ordem induzida em $\mathcal{A}$. Verificações rotineiras mostram que $\langle\mathcal{C}, \triangleleft\rangle$ é o.p. não-vazia (note que $\langle\emptyset, \emptyset\rangle \in \mathcal{C}$, por vacuidade) e satisfaz as condicões necessárias à aplicação do Lema de Zorn.)

Portanto, existe $\mathcal{T}=\left\{T_{\alpha}: \alpha<\zeta\right\} \subseteq[\omega]^{\omega}$ família "end-maximal"( $\triangleleft$-maximal), bem ordenada por $\supset^{*}$ e de tipo de ordem $\zeta$ para algum $\zeta$. É claro que $\zeta$ não pode ser sucessor. Suponha agora que $\mathcal{T}$ admita uma pseudo-intersecção infinita $A$; posso supor s.p.g. (sem perda de generalidade) que $A \in S$. É imediato que, nessas condições, $\mathcal{T}^{\prime}=\mathcal{T} \cup\{A\}$ seria uma subfamília de $S$ bem ordenada por $\supseteq^{*}$ e tal que $\mathcal{T} \triangleleft \mathcal{T}^{\prime}$, o que contradiz a maximalidade de $\mathcal{T}$. Portanto, $\mathcal{T}$ é uma torre.

Como torres possuem a s.f.i.p., é imediato que não existem torres enumeráveis, pois já sabemos que $\omega_{1} \leqslant \mathfrak{p}$. Poderíamos, porém, justificar esse fato com argumentos topológicos. Com efeito, seja $\mathcal{T}=\left\{T_{n}: n<\omega\right\}$ uma subfamília de $[\omega]^{\omega}$ bem ordenada por $\supset^{*}$. $\mathcal{T}$ induz naturalmente uma seqüência estritamente decrescente de clopens em $\beta \omega \backslash \omega$, a saber a seqüência de abertos básicos $\left\{W\left(T_{n}\right): n<\omega\right\}$, conforme terminologia utilizada no Capítulo 1. Essa sequência de clopens em $\beta \omega \backslash \omega$ atende à definição de "nested-sequence" que aparece em [Hec72]. Em particular, $\left\{W\left(T_{n}\right): n<\omega\right\}$ é uma família de fechados com a p.i.f. no espaço compacto $\beta \omega \backslash \omega$, logo sua intersecção não é vazia. Mas, destacamos entre os fatos básicos a respeito de $\beta \omega$ no capítulo anterior que, se a interseç̧ão de uma família enumerável de abertos de $\beta \omega \backslash \omega$ é não-vazia, ela contém um aberto não-vazio. Segue que existe $E \in[\omega]^{\omega}$ tal que $W(E) \subseteq \bigcap_{n<\omega} W\left(T_{n}\right)$. Consequentemente, $E \subseteq \subseteq^{*} T_{i}$ para todo $i<\omega$ e $E$ é uma pseudo-intersecção infinita de $\left\{T_{n}: n<\omega\right\}$.

\section{$2.2 \mathfrak{a}, \mathfrak{b}, \mathfrak{d}, \mathfrak{p}, \mathfrak{s}$ e $\mathfrak{t}$ em $Z \mathbb{F} C$}

O objetivo principal desta seção é demonstrar o seguinte

Teorema 2.1 Valem em ZFC as seguintes afirmações:

a) $\omega_{1} \leqslant \mathfrak{p} \leqslant \mathfrak{t} \leqslant \mathfrak{b} \leqslant \mathfrak{a}, \mathfrak{t} \leqslant \mathfrak{s} \leqslant \mathfrak{d}$ e $\mathfrak{b} \leqslant \mathfrak{d}$.

b) $\mathfrak{p}=\omega_{1}$ se e só se $\mathfrak{t}=\omega_{1}$.

c) Se $\omega \leqslant \kappa<\mathfrak{t}$, então $2^{\kappa}=\mathfrak{c}$. 
d) $\mathfrak{t} e \mathfrak{b}$ são cardinais regulares, $e \mathfrak{b} \leqslant c f(\mathfrak{d})$.

e) p é regular.

Durante a demonstração do teorema acima, notaremos que seriam úteis outras caracterizações para esses cardinais; isso justificará a seção seguinte. Porém, conseguiremos demonstrar o teorema sem deixar muitos resultados "em débito" ; apenas na demonstração de e) utilizaremos um fato que depende de um resultado da próxima seção.

No final desta seção, utilizaremos a regularidade de $\mathfrak{p}$ para, discutindo o conceito de $P$-pontos em $\beta \omega \backslash \omega=\omega^{*}$, entendermos o motivo pelo qual a letra "p germânica" foi escolhida para designar o pequeno cardinal $\mathfrak{p}$.

a) Prova de $\omega_{1} \leqslant \mathfrak{p}$ : Já feita na seção anterior.

Prova de $\mathfrak{p} \leqslant \mathfrak{t}$ : Imediata, já que torres são famílias com a s.f.i.p. e sem pseudointersecção infinita.

Prova de $\mathfrak{t} \leqslant \mathfrak{b}$ : Seja $\kappa<\mathfrak{t}$ um cardinal; mostraremos que qualquer subfamília de ${ }^{\omega} \omega$ com cardinalidade $\kappa$ não é ilimitada em ${ }^{\omega} \omega$, o que é suficiente para nós. Seja então $B=\left\{b_{\zeta}: \zeta<\kappa+1\right\} \subseteq{ }^{\omega} \omega$ (a enumeração até $\kappa+1$ é "slightly more convenient", como diria van Douwen). Construiremos por recursão em $\zeta<\kappa+1$ funções estritamente crescentes $f_{\zeta} \in{ }^{\omega} \omega$ satisfazendo, para cada $\zeta<\kappa+1$,

$$
\begin{aligned}
& (1)(\forall \xi<\zeta)\left[i m\left(f_{\zeta}\right) \subseteq^{*} i m\left(f_{\xi}\right)\right] ; \\
& (2)(\forall n<\omega)\left[f_{\zeta}(n) \geqslant \max \left\{b_{\zeta}(k): k \leqslant 2 n\right\}\right] .
\end{aligned}
$$

$f_{0}$ pode ser convenientemente construída. Seja agora $0<\zeta<\kappa+1$ e suponha que a família $\left\{f_{\xi}: \xi<\zeta\right\}$ esteja construída satisfazendo (1) e (2). Note que, de (1) e de $|\zeta| \leqslant \kappa<t,\left\{i m\left(f_{\xi}\right): \xi<\zeta\right\}$ admite uma pseudo-intersecção infinita $A$ (esse fato é bastante razoável, se observarmos que os elementos dessa família que pertencem a classes de equivalência distintas por $={ }^{*}$ formam um conjunto bem ordenado por $\supset^{*}$ e de cardinalidade menor que $\mathfrak{t}$; de todo modo, a igualdade " $t=\mathfrak{t}_{1}$ " a ser provada na próxima seção elimina a necessidade do "bastante razoável" acima). Definimos então $f_{\zeta}: \omega \mapsto \omega$ recursivamente, pondo, para cada $n<\omega$,

$$
f_{\zeta}(n)=\min \left\{a \in A:\left((\forall k \leqslant 2 n)\left[b_{\zeta}(k) \leqslant a\right]\right) \wedge\left(a>\sup \left(i m\left(f_{\zeta}\lceil n)\right)\right)\right\}\right.
$$


e $\operatorname{assim} f_{\zeta}$ é estritamente crescente e as condições (1) e (2) valem claramente.

Afirmamos agora que a última função construída, $f_{\kappa}$, é um limitante superior para $B$. De fato: seja $\xi<\kappa+1$ qualquer. Temos $f_{\kappa}$ injetora, estritamente crescente e $i m\left(f_{\kappa}\right) \subseteq^{*} i m\left(f_{\xi}\right)$, logo existe $m<\omega$ tal que $(\forall n<\omega)\left[f_{\kappa}(m+n) \in i m\left(f_{\xi}\right)\right]$. Mas, como $f_{\kappa}$ e $f_{\xi}$ são estritamente crescentes, segue que $(\forall n<\omega)\left[f_{\kappa}(m+n) \geqslant f_{\xi}(n)\right]$. Basta agora usar (2) e podemos afirmar que, se $n<\omega$ é tal que $n \geqslant 2 m$, então

$$
f_{\kappa}(n)=f_{\kappa}(n-m+m) \geqslant f_{\xi}(n-m) \geqslant b_{\xi}(n)
$$

pois $2 m \leqslant n$ nos dá $n \leqslant 2(n-m)$ e $\left(k \leqslant 2 n \Rightarrow b_{\xi}(k) \leqslant f_{\xi}(n)\right)$.

$\therefore(\forall \xi<\kappa+1)\left[b_{\xi} \leqslant{ }^{*} f_{\kappa}\right]$ e $f_{\kappa}$ é limitante superior de $B$.

Prova de $\mathfrak{b} \leqslant \mathfrak{a}$ : Seja $\mathcal{F} \subseteq{ }^{\omega} \omega$ uma família a.d. infinita com $|\mathcal{F}|=\kappa<\mathfrak{b}$; mostraremos que $\mathcal{F}$ não é maximal, exibindo $B \in[\omega]^{\omega}$ tal que $\mathcal{F} \cup\{B\}$ é a.d. Enumeramos $\mathcal{F}=\left\{A_{\alpha}\right.$ : $\alpha<\kappa\}$. Afirmamos que podemos supor que $\left\{A_{n}: n<\omega\right\}$ é efetivamente disjunta. De fato, a construção $A_{0}^{\prime}=A_{0}$ e $A_{n+1}^{\prime}=A_{n+1} \backslash \bigcup_{m \leqslant n} A_{m}=A_{n+1} \backslash \bigcup_{m \leqslant n} A_{n+1} \cap A_{m}$ mostra que podemos extrair uma quantidade no máximo finita de cada um dos elementos de $\left\{A_{n}: n<\omega\right\}$, obtendo $\left\{A_{n}^{\prime}: n<\omega\right\}$ disjunta. Assim, se exibirmos $B \in[\omega]^{\omega}$ tal que $\left|B \cap A_{n}^{\prime}\right|<\omega$ para todo $<\omega$, automaticamente teremos $\left|B \cap A_{n}\right|<\omega$ para todo $n<\omega$.

Pois bem. Temos $\mathcal{F}=\left\{A_{\alpha}: \alpha<\kappa\right\}$ a.d. e $\left\{A_{n}: n<\omega\right\}$ disjunta. Enumeramos agora cada um dos conjuntos $A_{n}$ pondo $A_{n}=\left\{a_{n, m}: m<\omega\right\}$ na (única) enumeração crescente. Para cada $\omega \leqslant \alpha<\kappa$, definimos $f_{\alpha} \in{ }^{\omega} \omega$ pondo

$$
f_{\alpha}(n)= \begin{cases}m & \text { se } \max \left(A_{\alpha} \cap A_{n}\right)=a_{n, m} \\ 0 & \text { se } A_{\alpha} \cap A_{n}=\emptyset\end{cases}
$$

Como $\kappa<\mathfrak{b}$, existe $f \in \omega^{\omega} \omega$ tal que $f_{\alpha} \leqslant{ }^{*} f$ para todos os $\alpha$ 's com $\omega \leqslant \alpha<\kappa$; posso ainda supor, s.p.g., que tal f satisfaz, para cada $\omega \leqslant \alpha<\kappa$, não só $\mid\{n<\omega: f(n)<$ $\left.f_{\alpha}(n)\right\} \mid<\omega$ como também $\left|\left\{n<\omega: f(n) \leqslant f_{\alpha}(n)\right\}\right|<\omega$ (o que se obtém "somando 1", se necessário, a todos os valores de uma função limitante de $\left.\left\{f_{\alpha}: \omega \leqslant \alpha<\kappa\right\}\right)$. Seja então $B=\left\{a_{n, f(n)}: n<\omega\right\}$. Temos $B$ infinito, pois os conjuntos $A_{n}$ são disjuntos, e vale que $B \cap A_{n}$ é finito (na verdade, unitário) para cada $n<\omega$. Se $\alpha \geqslant \omega$, da suposição acima podemos afirmar que $B \cap A_{\alpha}$ é finito, pois $a_{n, f(n)} \in A_{\alpha}$ somente se $f(n) \leqslant m=f_{\alpha}(n)$. 
Assim, obtemos um conjunto $B$ nas condições desejadas.

Prova de $\mathfrak{t} \leqslant \mathfrak{s}$ : Seja $\kappa<\mathfrak{t}$; mostraremos que uma família $\mathcal{S} \subseteq[\omega]^{\omega}$ tal que $|\mathcal{S}|=\kappa$ não pode ser splitting, o que nos garante o desejado. Enumeramos $\mathcal{S}$ na forma $\left\{\mathcal{S}_{\zeta}: \zeta<\kappa+1\right\}$ e, por recursão transfinita sobre $\zeta<\kappa+1$ construimos uma família $\left\{T_{\zeta}: \zeta<\kappa+1\right\} \subseteq$ ${ }^{\omega} \omega$ satisfazendo

$$
\begin{aligned}
& (3)(\forall \xi<\zeta)\left[T_{\zeta} \subseteq^{*} T_{\xi}\right] \\
& (4)\left(T_{\zeta} \subseteq S_{\zeta}\right) \vee\left(T_{\zeta} \subseteq\left(\omega \backslash S_{\zeta}\right)\right)
\end{aligned}
$$

Podemos tomar $T_{0}=S_{0}$. Supondo construídas as $T_{\xi}$ para $\xi<\zeta$, de $|\zeta| \leqslant \kappa<\mathfrak{t}$ podemos tomar uma pseudo-intersecção infinita $A$ de $\left\{T_{\xi}: \xi<\zeta\right\}$, analogamente ao que fizemos na prova de $\mathfrak{t} \leqslant \mathfrak{b}$. Se $A \cap S_{\zeta}$ é infinito, definimos $T_{\zeta}=A \cap S_{\zeta}$, caso contrário pomos $T_{\zeta}=A \backslash S_{\zeta}$, e as condições desejadas valem obviamente. Segue agora que $\mathcal{S}$ não "splits" $T_{\kappa}$, já que $(\forall S \in \mathcal{S})\left[\left(T_{\kappa} \subseteq^{*} S\right) \vee\left(T_{\kappa} \subseteq^{*} \omega \backslash S\right)\right]$ donde $\mathcal{S}$ não é splitting.

Prova de $\mathfrak{s} \leqslant \mathfrak{o}$. Para cada $A \in[\omega]^{\omega}$, denotamos $b_{A}: \omega \mapsto A$ a (única) enumeração crescente de $A$. Para cada $f \in{ }^{\omega} \omega$, definimos recursivamente $f^{0}=I$ (função identidade) e $f^{n+1}=f^{n} \circ f$. Se $f$ é uma função estritamente crescente de $\omega$ em $\omega$ e com $f(0) \neq 0$, ou mesmo se $f$ satisfaz apenas $(\forall n<\omega)\left[f^{n}(0)<f^{n+1}(0)\right]$, definimos

$$
S_{f}=\bigcup_{n<\omega}\left[f^{2 n}(0), f^{2 n+1}(0)[\quad \text { intervalos em } \omega)\right.
$$

Temos então o seguinte

Fato 2.1 Se $A \in[\omega]^{\omega}$ e $\mathcal{F}$ é uma família dominante formada por funções estritamente crescentes com $f(0) \neq 0$, então existe $f \in \mathcal{F}$ tal que $S_{f}$ "splits" $A$, isto é, tal que $\left|A \cap S_{f}\right|=$ $\left|A \backslash S_{f}\right|=\omega$.

Verifiquemos a veracidade do fato 2.1. Como $\mathcal{F}$ é dominante, podemos supor que existe $f \in \mathcal{F}$ tal que $b_{A}<^{*} f$, e, mais ainda, assumimos s.p.g. que para um certo $m<\omega$ valha que $(\forall n \geqslant m)\left[b_{A}(n)<f(n)\right]$. Como $b_{A}$ é estritamente crescente, vale também que $(\forall n<\omega)\left[n \leqslant b_{A}(n)\right]$. Segue que para $n \geqslant m$ tem-se $f^{n}(0) \geqslant n \geqslant m$ e

$$
f^{n}(0) \leqslant b_{A}\left(f^{n}(0)\right)<f\left(f^{n}(0)\right)=f^{n+1}(0)
$$


e agora é imediato que, para $n \geqslant m$, o $f^{n}(0)$-ésimo elemento de $A$ pertence a $S_{f}$ ou $\omega \backslash S_{f}$ conforme $n$ seja par ou impar.

Com o fato 2.1, o resultado segue imediatamente: tome $\mathcal{F} \subseteq{ }^{\omega} \omega$ uma família dominante com $|\mathcal{F}|=\mathfrak{d}$ e assuma que todas as funções de $\mathcal{F}$ são estritamente crescentes e com $f(0) \neq 0$. Então $\left\{S_{f}: f \in \mathcal{F}\right\}$ é splitting e $\mathfrak{s} \leqslant \mathfrak{d}$.

Prova de $\mathfrak{b} \leqslant \mathfrak{d}$. Imediata, pois toda família dominante em ${ }^{\omega} \omega$ é ilimitada.

Um diagrama que representa a relação de ordem em $Z F C$ existente entre os cardinais $\omega_{1}, \mathfrak{a}, \mathfrak{b}, \mathfrak{d}, \mathfrak{p}, \mathfrak{s}, \mathfrak{t}$ e $\mathfrak{c}$ pode ser visto nas páginas iniciais deste trabalho, um pouco antes do abstract.

Prova de $b$ ). Se $\mathfrak{t}=\omega_{1}, \mathfrak{p}=\omega_{1}$ claramente; resta verificar que $\mathfrak{p}=\omega_{1}$ implica $\mathfrak{t}=\omega_{1}$. Para isso, mostraremos que se $\mathfrak{t}>\omega_{1}$ então $\mathfrak{p}>\omega_{1}$.

Assuma $\omega_{1}<\mathfrak{t}$. Então, se $\mathcal{F} \subseteq{ }^{\omega} \omega,|\mathcal{F}| \leqslant \omega_{1}$, a desigualdade $\omega_{1}<\mathfrak{t} \leqslant \mathfrak{b}$ nos dá que existe $g \in{ }^{\omega} \omega$ tal que $f \leqslant{ }^{*} g$ para toda $f \in \mathcal{F}$. Usaremos essa observação na demonstração do seguinte

Lema $2.1\left[\omega_{1}<\mathfrak{t}\right]$

Suponha que $\mathcal{C}$ e $\mathcal{B}$ são subfamílias de $[\omega]^{\omega}$, com $|\mathcal{C}| \leqslant \omega_{1},|\mathcal{B}| \leqslant \omega$ e tais que $C \subseteq^{*} B$ sempre que $C \in \mathcal{C}$ e $B \in \mathcal{B}$. Então existe $D \in[\omega]^{\omega}$ tal que $C \subseteq^{*} D \subseteq^{*} B$ para quaisquer $C \in \mathcal{C}$ e $B \in \mathcal{B}$.

Prova do Lema: Se $\mathcal{B}=\emptyset$, tome $D=\omega$. Se $\mathcal{B}$ é finito, tome $D=\bigcap \mathcal{B}$. Se $|\mathcal{B}|=\omega$, enumere $\mathcal{B}=\left\{B_{n}: n<\omega\right\}$ e defina $B_{n}^{\prime}=\bigcap_{i \leqslant n} B_{i}$. Tem-se $C \subseteq^{*} B_{n}^{\prime}$ para quaisquer $C \in \mathcal{C}$ e $n<\omega$. Se existir $n<\omega$ tal que $B_{n}^{\prime} \subseteq^{*} B_{m}^{\prime}$ para todo $m<\omega$, podemos tomar $D=B_{n}^{\prime}$. Caso contrário, existe uma sequência estritamente crescente $\left\langle n_{k}: k<\omega\right\rangle$ tal que

$$
B_{n_{0}}^{\prime} \nsubseteq^{*} B_{n_{1}}^{\prime} \nsubseteq^{*} \ldots \nsubseteq^{*} B_{n_{k}}^{\prime} \nsubseteq^{*} \ldots
$$

logo, se definirmos, para todo $k<\omega, D_{k}=B_{n_{k}}^{\prime} \backslash B_{n_{k+1}}^{\prime}$, obteremos uma família $\left\{D_{k}\right.$ : $k<\omega\} \subseteq[\omega]^{\omega}$.

Para cada $\kappa<\omega$ e para cada $C \in \mathcal{C}$ temos $D_{k} \cap C \subseteq C \backslash B_{n_{k+1}}^{\prime}, \log D_{k} \cap C$ é finito: defino então uma família de funções $\mathcal{F}=\left\{f_{C}: C \in \mathcal{C}\right\}$ pondo, para cada $k<\omega$ e para 
cada $C \in \mathcal{C}, f_{C}(k)=\max \left(D_{k} \cap C\right)$ se $D_{k} \cap C \neq \emptyset$ e $f_{C}(k)=0$ se $D_{k} \cap C=\emptyset$. De $|\mathcal{F}| \leqslant \omega_{1}$ e da observação antes do lema, existe uma função $g \in{ }^{\omega} \omega$ tal que $\left\{n<\omega: g(n)<f_{c}(n)\right\}$ é finito para todo $C \in \mathcal{C}$.

Seja agora

$$
D=B_{n_{0}}^{\prime} \backslash\left(\bigcup_{k<\omega}\left\{i: i \in D_{k}, g(k)<i\right\}\right)
$$

Afirmamos que tal conjunto satisfaz o desejado.

De fato: se $C \in \mathcal{C}, C \subseteq \subseteq^{*} B_{n_{0}}^{\prime}$ e $C \cap\left(\bigcup_{k<\omega}\left\{i: i \in D_{k}, g(k)<i\right\}\right)$ é finito, pois

$$
C \cap\left(\bigcup_{k<\omega}\left\{i: i \in D_{k}, g(k)<i\right\}\right)=\bigcup_{k<\omega}\left\{i: i \in D_{k} \cap C, g(k)<i\right\}
$$

e a definição da $f_{C}$ e o fato de $\left\{n<\omega: g(n)<f_{C}(n)\right\}$ ser finito implicam que o conjunto acima é uma união finita de conjuntos finitos. Logo, $C \subseteq^{*} D$ para todo $C \in \mathcal{C}$. Por outro lado, será fácil mostrar que $D \subseteq \subseteq^{*} B_{n_{k}}^{\prime}$ para todo $k<\omega$, o que garantirá o resultado. A prova é por indução em $k$. Se $k=0$, é imediato. Suponha agora $D \subseteq^{*} B_{n_{k}}^{\prime}$, isto é, suponha $\left|D \backslash B_{n_{k}}^{\prime}\right|<\omega$. Note agora que $D=D \backslash\left\{i: i \in D_{k}, g(k)<i\right\}$ e que $\left\{i: i \in D_{k}, g(k)<i\right\}={ }^{*} D_{k}$. Portanto

$$
\begin{aligned}
& D \backslash B_{n_{k+1}}^{\prime}=\left(D \backslash\left\{i: i \in D_{k}, g(k)<i\right\}\right) \backslash B_{n_{k+1}}^{\prime} \\
& ={ }^{*}\left(D \backslash D_{k}\right) \backslash B_{n_{k+1}}^{\prime} \\
& =D \backslash\left(D_{k} \cup B_{n_{k+1}}^{\prime}\right) \\
& =D \backslash B_{n_{k}}^{\prime}
\end{aligned}
$$

donde também vale $\left|D \backslash B_{n_{k+1}}^{\prime}\right|<\omega$ e a indução está completa, e o lema demonstrado.

Mostremos então que $\mathfrak{p}>\omega_{1}$ se $\mathfrak{t}>\omega_{1}$. Seja $\mathcal{A} \subseteq[\omega]^{\omega}$ qualquer, $\operatorname{com}|\mathcal{A}| \leqslant \omega_{1}$ e tal que $\mathcal{A}$ possui a s.f.i.p. ; se $\mathcal{A}$ for finito ou enumerável, a existência de pseudointersecção infinita é imediata, a partir do que já vimos na seção anterior. Podemos supor então $|\mathcal{A}|=\omega_{1}$. Como era de se esperar, construiremos uma pseudo-intersecção infinita para $\mathcal{A}$, o que garantirá $\mathfrak{p}>\omega_{1}$. Enumeramos $\mathcal{A}=\left\{A_{\zeta}: \zeta<\omega_{1}\right\}$ e denotamos por $\mathcal{A}^{*}=\left\{\cap \mathcal{F}: \mathcal{F} \in[\mathcal{A}]^{<\omega}\right\}$ o conjunto de todas as intersecções finitas de elementos de $\mathcal{A}$; por hipótese, $\mathcal{A}^{*} \subseteq\left[\left.\omega\right|^{\omega}\right.$ também. Vamos construir recursivamente uma família $\mathcal{B}=\left\{B_{\zeta}: \zeta<\omega_{1}\right\}$ satisfazendo, para cada $\zeta<\omega_{1}$,

$$
(5)(\forall \xi<\zeta)\left[B_{\zeta} \subseteq^{*} B_{\xi}\right]
$$


(6) $B_{\zeta+1} \subseteq A_{\zeta}$

$(7)\left(\forall F \in \mathcal{A}^{*}\right)\left[\left|B_{\zeta} \cap F\right|=\omega\right]$

A justificativa da construção é como segue:

$\zeta=0$ : Tome $B_{0}=\omega$.

$\zeta$ ordinal sucessor: Suponha $\zeta=\alpha+1$ e que os $B_{\xi}$ 's construídos para $\xi \leqslant \alpha$ satisfaçam as hipóteses desejadas. Então, definimos $B_{\zeta}=B_{\alpha} \cap A_{\alpha}$ e temos $B_{\alpha+1}=B_{\zeta} \subseteq$ $A_{\alpha}$; e , se $F$ é intersecção finita de elementos de $\mathcal{A}$, temos $B_{\zeta} \cap F=\left(B_{\alpha} \cap A_{\alpha}\right) \cap F=$ $B_{\alpha} \cap\left(A_{\alpha} \cap F\right)$, que é infinito por hipótese de indução ( e este é o momento em que $\mathcal{A}^{*}$ se faz necessário). Vale também para todo $\xi<\zeta$ ( que são os $\xi \leqslant \alpha$ ) que $B_{\zeta}=B_{\alpha+1} \subseteq B_{\alpha} \subseteq^{*} B_{\xi}$. Assim, $B_{\zeta}=B_{\alpha} \cap A_{\alpha}$ satisfaz o desejado.

$\zeta$ ordinal limite: Suponha construída a família $\left\{B_{\xi}: \xi<\zeta\right\}$ onde $\zeta<\omega_{1}$ é ordinal limite. Temos então uma $\supseteq^{*}$-boa ordem de tipo de ordem $\zeta$

$$
B_{0} \supseteq^{*} B_{1} \supseteq^{*} \ldots \supseteq^{*} B_{\xi} \supseteq^{*} \ldots
$$

e, consequentemente, para cada $F \in \mathcal{A}^{*}$ temos uma $\supseteq^{*}$-boa-ordem de tipo de ordem $\zeta$

$$
B_{0} \cap F \supseteq^{*} B_{1} \cap F \supseteq^{*} \ldots B_{\xi} \cap F \supseteq^{*} \ldots
$$

que, por ser enumerável, admite uma pseudo-intersecção infinita $C_{F}$, que podemos supor estar contida em $F$, e assim $C_{F} \subseteq^{*} B_{\xi}$ para todo $\xi<\zeta$.

Então temos $\left\{B_{\xi}: \xi<\zeta\right\}$ enumerável, $\left|\left\{C_{F}: F \in \mathcal{A}^{*}\right\}\right| \leqslant\left|\mathcal{A}^{*}\right| \leqslant|\mathcal{A}|=\omega_{1}$ e vale que $C_{F} \subseteq^{*} B_{\xi}$ para todos $F \in \mathcal{A}^{*}$ e $\xi<\zeta$. Segue então do Lema 2.1 que existe um determinado conjunto , $B_{\zeta} \in[\omega]^{\omega}$ digamos, satisfazendo $C_{F} \subseteq^{*} B_{\zeta} \subseteq^{*} B_{\xi}$ para todos os $F \in \mathcal{A}^{*}$ e $\xi<\zeta$. O primeiro " $\subseteq$ *" garante (7), o segundo garante (5) e não temos que nos preocupar com (6) no caso de $\zeta$ limite. Portanto, a recursão está bem definida.

Construída então a família $\mathcal{B}=\left\{B_{\zeta}: \zeta<\omega_{1}\right\}$, a hipótese $\omega_{1}<\mathfrak{t}$ nos dá um conjunto $I \in[\omega]^{\omega}$, pseudo-interseç̧ão infinita de $\mathcal{B}$. Temos então $I \subseteq^{*} B_{\zeta+1} \subseteq^{*} A_{\zeta}$ para todo $\zeta<\omega_{1}$, donde $I$ é pseudo-intersecção infinita de $\mathcal{A}$ e obtivemos o desejado.

Prova de $c$ ): Seja $\kappa$ tal que $\omega \leqslant \kappa<\mathfrak{t}$. Então $c \leqslant 2^{\kappa}$, logo basta mostrar que $2^{\kappa} \leqslant \mathfrak{c}$. Para isso, mostraremos que, se $\zeta<\kappa+1$, então existe uma família $\left\{T_{f}: f \in{ }^{\zeta} 2\right\} \subseteq[\omega]^{\omega}$ satisfazendo

$$
(8)\left(\forall f \in{ }^{\varsigma_{2}}\right)(\forall \xi<\zeta)\left[T_{f} \subseteq^{*} T_{f \mid \xi}\right]
$$


$(9)\left(\forall f, g \in \varsigma^{\varsigma} 2\right)\left[f \neq g \Rightarrow T_{f} \cap T_{g}\right.$ é finito $]$.

Observamos que, no caso particular de $\zeta=\kappa$ em (9), temos que a aplicação $f \in$ $\kappa_{2} \mapsto T_{f} \in[\omega]^{\omega}$ é injetora, donde segue o desejado.

A construção das famílias $\left\{T_{f}: f \in \varsigma^{\zeta}\right\}$ é feita por recursão em $\zeta<\kappa+1$. Se $\zeta=0$, então ${ }^{0} 2=\{\emptyset\}$ e posso tomar $T_{\emptyset}=\omega$. Seja agora $0<\zeta<\kappa+1$ e assuma que as famílias $\left\{T_{f}: f \in \xi^{\xi} 2\right\}$ estejam bem construídas para todo $\xi<\zeta$. Temos 2 casos a considerar: se $\zeta$ é ordinal sucessor, digamos $\zeta=\delta+1$, para cada $f \in{ }^{\delta} 2$ decompomos $T_{f}$ em dois subconjuntos disjuntos e infinitos, $T_{f, 0}$ e $T_{f, 1}$. Daí, para cada $g \in \varsigma_{2}$, defino $T_{g}=T_{g \mid \delta, g(\delta)}$. É imediato que a condição $(8)$ está satisfeita (note que, se $\xi<\delta$, então $(f \uparrow \delta)|\xi=f| \xi)$. Se $\zeta$ é ordinal limite, usamos que $|\zeta| \leqslant \kappa<\mathfrak{t}$ e tomamos, para cada $f \in \varsigma_{2}, T_{f}$ uma pseudo-intersecção infinita de $\left\{T_{f \mid \xi}: \xi<\zeta\right\}$. ( $(\delta)$ está claramente satisfeita. Para verificar (9) em ambos os casos, tome $f$ e $g$ distintas em $\varsigma_{2}$ e considere $\beta=\min \{\xi<\zeta: f(\xi) \neq g(\xi)\}$. Se $\beta+1=\zeta$, a construção feita para o caso " $\zeta$ sucessor" garante que $T_{f} \cap T_{g}$ é vazio. Se $\beta+1<\zeta$ (o que pode ocorrer no "caso sucessor" e sempre ocorre no "caso limite"), temos que $T_{f \mid \beta+1} \cap T_{g \mid \beta+1}$ é finito por hipótese de indução donde $T_{f} \cap T_{g}$ é finito, pois $T_{f} \subseteq^{*} T_{f \mid \beta+1}$ e $T_{g} \subseteq^{*} T_{g \mid \beta+1} \operatorname{logo} T_{f} \cap T_{g} \subseteq^{*} T_{f \mid \beta+1} \cap T_{g \mid \beta+1}$.

Prova de $d$ ): A regularidade de $\mathfrak{t}$ é intuitivamente clara: formalizemos essa idéia. Seja $\left\{T_{\alpha}: \alpha<\beta\right\}$ uma torre de tipo de ordem $\beta$ (com a enumeração dada pelo tipo de ordem) e de tamanho mínimo, isto é. $|\beta|=\mathrm{t}$. Como já comentamos em outra oportunidade, é evidente que $\beta$ é ordinal limite. Seja $\lambda=c f(\beta)$; sabemos que existe então uma seqüência $\left\langle\alpha_{\xi}: \xi<\lambda\right\rangle$ estritamente crescente e cofinal em $\beta$, e temos que $\left\{T_{\alpha_{\xi}}: \xi<\lambda\right\}$ é uma torre, já que é claramente bem-ordenada por $\supset^{*}$ e um conjunto $A \in[\omega]^{\omega}$ que fosse pseudointersecção infinita de $\left\{T_{\alpha_{\xi}}: \xi<\lambda\right\}$ seria também pseudo-intersecção infinita de $\left\{T_{\alpha}\right.$ : $\alpha<\beta\}$ (pois, nesse caso, para um dado $\zeta<\beta$ qualquer bastaria tomar $\xi<\lambda$ tal que $\zeta<\alpha_{\xi}$ e teríamos $\left.T_{\zeta} \supset^{*} T_{\alpha_{\xi}} \supseteq^{*} A\right)$ e isso o contradiria o fato de $\left\{T_{\alpha}: \alpha<\beta\right\}$ ser uma torre. Portanto, $\mathfrak{t} \leqslant c f(\beta) \leqslant|\beta|=\mathfrak{t} \operatorname{logo} c f(\beta)=\mathfrak{t}$ e mostramos o desejado, já que a cofinalidade de qualquer ordinal limite é um cardinal regular. Note também que a demonstração acima nos permite sempre supor, caso necessitemos, que uma torre de cardinalidade mínima tem, além de cardinalidade $t$, tipo de ordem $t$. 
Para a regularidade de $\mathfrak{b}$, afirmamos que $\mathfrak{b}=\mathfrak{b}_{1}$, onde

$\mathfrak{b}_{1}=\min \left\{|B|: B\right.$ é um subconjunto ilimitado de ${ }^{\omega_{\omega}}$ formado por funções estritamente crescentes e que é bem ordenado por $\left.<^{*}\right\}$

A desigualdade $\mathfrak{b} \leqslant \mathfrak{b}_{1}$ é clara. Já $\mathfrak{b}_{1} \leqslant \mathfrak{b}$ vem da seguinte construção recursiva: seja $F=\left\{f_{\zeta}: \zeta<\mathfrak{b}\right\}$ um subconjunto ilimitado de ${ }^{{ }^{\omega}} \omega$ e seja $S$ o conjunto $\left\{f \in{ }^{\omega} \omega: f\right.$ é estritamente crescente \}. S é claramente dominante. Construímos então uma família $\left\{g_{\zeta}: \zeta<\mathfrak{b}\right\} \subseteq S$ satisfazendo para todo $\zeta<\mathfrak{b}$

(10) $\forall f \in\left\{g_{\xi}: \xi<\zeta\right\} \cup\left\{f_{\zeta}\right\}\left[f<{ }^{*} g_{\zeta}\right]$

Escolhemos $g_{0}$ em $S$ que satisfaça $f_{0}<^{*} g_{0}$. Para $0<\zeta<\mathfrak{b}$ supomos construídas as funções $g_{\xi}$ satisfazendo (10) para todo $\xi<\zeta$. De $|\zeta|<\mathfrak{b}$ temos que $\mathcal{G}_{\zeta}=\left\{g_{\xi}: \xi<\zeta\right\} \cup\left\{f_{\zeta}\right\}$ é limitada, isto é, existe $g \in{ }^{\omega} \omega$ tal que a $\left(\forall f \in \mathcal{G}_{\zeta}\right)\left[f \leqslant{ }^{*} g\right]$. Agora, tomo $g_{\zeta} \in S$ satisfazendo $(\forall n<\omega)\left[g(n)<g_{\zeta}(n)\right]$. Então, $g<^{*} g_{\zeta},(10)$ vale para $\zeta$ e $\left\{g_{\zeta}: \zeta<\mathfrak{b}\right\}$ está construída. Note agora que um limitante superior de $\left\{g_{\zeta}: \zeta<\mathfrak{b}\right\}$ seria também um limitante superior de $F$, logo $\left\{g_{\zeta}: \zeta<\mathfrak{b}\right\}$ é uma família ilimitada e que está claramente bem-ordenada por $<^{*}$. Daí $\mathfrak{b}_{1} \leqslant \mathfrak{b}$ e a igualdade está demonstrada.

A partir daí, a demonstração de que b é regular é análoga àquela que fizemos para t: tomo uma família bem-ordenada por $<*$ formada por funções estritamente crescentes com tipo de ordem $\beta$ com $|\beta|=\mathfrak{b}_{1}=\mathfrak{b}$. Trabalhando com uma sequência cofinal e estritamente crescente em $\beta$, obtemos $\mathfrak{b} \leqslant c f(\beta) \leqslant|\beta|=\mathfrak{b}$ e $\mathfrak{b}$ é cardinal regular.

A seguinte observação terá grande importância na próxima seção.

Observação 2.1 Sempre que $B$ for um subconjunto ilimitado de ${ }^{\omega} \omega$ formado por funções estritamente crescentes, bem-ordenado por $<^{*}$ e de tamanho minimo (i.e., $|B|=\mathfrak{b}=\mathfrak{b}_{1}$ ), podemos supor sem perda de generalidade que $B$, enquanto subfamília de $[\omega \times \omega]^{\omega}$, é a.d.

De fato: analisemos a construção recursiva que fizemos acima. No passo em que se constrói a função $g_{\zeta}$, temos que g é um limitante superior de $\mathcal{G}_{\zeta}=\left\{g_{\xi}: \xi<\right.$ $\zeta\} \cup\left\{f_{\zeta}\right\}$ e construimos $g_{\zeta}$ que satisfizesse $(\forall n<\omega)\left[g(n)<g_{\zeta}(n)\right]$, com $g_{\zeta} \in S$. Note que isso implica que, para qualquer $\xi<\zeta,\left\{n<\omega: g_{\xi}(n)=g_{\zeta}(n)\right\}$ seja finito (já que $(\forall \xi<\zeta)\left[\left\{n<\omega: g(n)<g_{\xi}(n)\right\}\right.$ é finito $\left.]\right)$. Daí é imediato que, para quaisquer $\xi, \zeta<\mathfrak{b}$, 
$\left\{n<\omega: g_{\zeta}(n)=g_{\xi}(n)\right\}$ é sempre finito. Assim, uma construção satisfazendo a observação 2.1 é sempre possível.

A demonstração que fizemos para $\mathfrak{b}=\mathfrak{b}_{1}$ tem ainda um interessante corolário. Recordamos ao leitor que uma escala em $^{\omega} \omega$ é um subconjunto dominante e bem-ordenado por $\leqslant *$.

Corolário (da demonstração de $\left.\mathfrak{b}=\mathfrak{b}_{1}\right) \quad \mathfrak{b}=\mathfrak{d} \Longleftrightarrow{ }^{\omega} \omega$ possui uma escala.

Demonstração (do Corolário) $(\Rightarrow)$ Se $\mathfrak{b}=\mathfrak{d}$, aplico a construção recursiva que fizemos na prova de $\mathfrak{b}=\mathfrak{b}_{1}$ para uma família $F$ dominante de tamanho $\mathfrak{d}$. A família $\left\{g_{\zeta}: \zeta<\mathfrak{d}\right\}$ que se obtém nessa situação é claramente uma escala.

$(\Leftarrow)$ Afirmamos que, se existe uma escala em ${ }^{\omega_{\omega}} \omega$, então existe uma escala de tamanho (e tipo de ordem!) $\mathfrak{d}$. De fato, seja $E=\left\{e_{\alpha}: \alpha<\beta\right\}$ uma escala em ${ }^{\omega} \omega$, enumerada pelo seu tipo de ordem, e $D=\left\{d_{\zeta}: \zeta<\mathfrak{d}\right\} \subseteq{ }^{\omega} \omega$ uma família dominante. Construímos por recursão em $\zeta<\mathfrak{d}$ uma seqüência $\left\langle\alpha_{\zeta}: \zeta<\mathfrak{d}\right\rangle$ estritamente crescente em $\beta$ e satisfazendo, para todo $\zeta<\mathfrak{0}$

$$
\text { (11) } \forall f \in\left\{e_{\alpha_{\xi}}: \xi<\zeta\right\} \cup\left\{d_{\zeta}\right\}\left[f \leqslant{ }^{*} e_{\alpha_{\zeta}}\right]
$$

Tomamos $\alpha_{0}<\beta$ qualquer que satisfaça $d_{0} \leqslant{ }^{*} e_{\alpha_{0}} ;$ isso é possível, pois $E$ é escala e portanto dominante. Para $0<\zeta<\mathfrak{D}$, suponha construídos os $\alpha_{\xi}$ 's para todo $\xi<\zeta$ de modo que $\left\langle\alpha_{\xi}: \xi<\zeta\right\rangle$ seja uma seqüência crescente em $\beta$ e tome $\alpha^{\prime}<\beta$ que satisfaça $d_{\zeta} \leqslant{ }^{*} e_{\alpha^{\prime}}$. Note agora que $\delta=\sup \left\{\alpha_{\xi}: \xi<\zeta\right\}<\beta$; esse fato é óbvio para o caso em que $\zeta$ é sucessor e para o caso limite observe que, caso contrário, teríamos $\left\{e_{\alpha_{\xi}}: \xi<\zeta\right\}$ cofinal em $E$ e portanto dominante, o que é um absurdo pois $|\zeta|<0$. Faça $\eta=\max \left\{\delta+1, \alpha^{\prime}\right\}$. Então podemos tomar $\alpha_{\zeta}=\eta$ e a construção está bem definida. É imediato agora que $\left\{e_{\alpha_{\zeta}}: \zeta<\mathfrak{O}\right\}$ é uma escala em ${ }^{\omega} \omega$, enumerada pelo seu tipo de ordem.

Assumindo que existe uma escala de tipo de ordem o concluíriamos facilmente que $\mathfrak{o}$ é cardinal regular (pois qualquer subconjunto cofinal de uma escala é dominante); assim, é imediato que, se $\mathcal{E}$ é nossa escala de tipo de ordem $\mathfrak{d}$, então nenhuma subfamília de $\mathcal{E}$ com cardinalidade estritamente menor que $\mathfrak{d}$ pode ser ilimitada; daí segue que, assumindo a existência de uma escala com tipo de ordem $\partial$, toda subfamília de ${ }^{\omega} \omega$ de cardinalidade 
menor que $\mathfrak{d}$ é limitada.

Resta mostrar ainda que $\mathfrak{b} \leqslant c f(\mathfrak{d})$. Seja então $\left\{f_{\zeta}: \zeta<\mathfrak{o}\right\}$ uma família dominante em ${ }^{\omega} \omega$, e seja $\left\langle\zeta_{\xi}: \xi<\lambda\right\rangle$ uma sequência crescente e cofinal em $\mathfrak{d}$, com $\lambda=c f(\mathfrak{d})$ e $\zeta_{0}=0$. Construímos por recursão transfinita uma família de funções $\left\{g_{\xi}: \xi<\lambda\right\} \subseteq{ }^{\omega} \omega$ satisfazendo, para todo $\xi<\lambda$

(12) $\forall f \in\left\{f_{\alpha}: \alpha \leqslant \zeta_{\xi}\right\} \cup\left\{g_{\beta}: \beta<\xi\right\}\left[g_{\xi} \not^{*} f\right]$

A função $g_{0}$ pode ser convenientemente construída. Seja agora $0<\xi<\lambda$ e suponha que as $g_{\beta}$ 's estejam bem construídas para todo $\beta<\xi$. Então $\left|\zeta_{\xi}\right|+|\xi|<\mathfrak{d}$ e $\left\{f_{\alpha}: \alpha \leqslant \zeta_{\xi}\right\} \cup\left\{g_{\beta}: \beta<\xi\right\}$ não é uma família dominante, logo existe uma função $g_{\xi} \in$ ${ }^{\omega} \omega$ que satisfaz o desejado, sendo $g_{\xi} \neq g_{\beta}$ sempre que $\beta<\xi$.

Afirmamos que $\left\{g_{\xi}: \xi<\lambda\right\}$ é uma família ilimitada de ${ }^{\omega} \omega$. De fato: suponhamos por absurdo que $h$ é um limitante superior de $\left\{g_{\xi}: \xi<\lambda\right\}$. Como $\left\{f_{\zeta}: \zeta<\mathfrak{d}\right\}$ é dominante, existe $\alpha<\mathfrak{d}$ tal que $h \leqslant^{*} f_{\alpha}$. Tomamos agora $\beta<\lambda$ tal que $\alpha<\zeta_{\beta}$. Então, por (12), $g_{\beta} \mathbb{k}^{*} f_{\alpha}$, o que contradiz $g_{\beta} \leqslant \leqslant^{*} h \leqslant f_{\alpha}$. Assim, $\mathfrak{b} \leqslant \lambda=c f(\mathfrak{d})$. Observamos que nada pode nos garantir que $\left\{g_{\xi}: \xi<\lambda\right\}$ é dominante.

Prova de e): Para provarmos que p é regular, assumiremos momentaneamente o seguinte Fato 2.2 Se $\mathcal{A}, \mathcal{B} \subseteq[\omega]^{\omega}$ satisfazem $|\mathcal{A}|+|\mathcal{B}|<\mathfrak{p}$ e se cada elemento de $\mathcal{B}$ é uma pseudo-intersecção de $\mathcal{A}$, então $\mathcal{A}$ possui uma pseudo-intersecção infinita I satisfazendo $(\forall B \in \mathcal{B})[B \cap I$ é infinito $]$.

O fato acima é consequência imediata da igualdade $\mathfrak{p}=\mathfrak{p}_{2}$ a ser apresentada na seção seguinte.

Pois bem; mostraremos que $\mathfrak{p}$ é regular verificando que, se uma subfamília $\mathcal{F}$ de $[\omega]^{\omega}$ possui a s.f.i.p. e é tal que $|\mathcal{F}|$ é singular e $|\mathcal{F}| \leqslant \mathfrak{p}$, então $\mathcal{F}$ possui uma pseudointersecção infinita.

Seja então $\mathcal{F}$ como no parágrafo anterior; é conveniente supor que $\mathcal{F}$ é fechada para intersecções finitas. Como assumimos que $|\mathcal{F}|$ é singular, podemos afirmar que existe $\kappa<|\mathcal{F}| \leqslant \mathfrak{p}$ tal que

$$
\mathcal{F}=\bigcup_{\delta<\kappa} \mathcal{F}_{\delta} \quad \operatorname{com}(\forall \delta<\kappa)\left[\left|\mathcal{F}_{\delta}\right|<\mathfrak{p}\right]
$$


Construímos agora uma subfamília de $[\omega]^{\omega},\left\{T_{\zeta}: \zeta<\kappa\right\}$, satisfazendo, para todo $\zeta<\kappa$,

(13) $T_{\zeta}$ é pseudo-intersecção infinita de $\mathcal{F}_{\zeta}$

(14) $(\forall \xi<\zeta)\left[T_{\zeta} \subseteq^{*} T_{\xi}\right]$

(15) $(\forall F \in \mathcal{F})\left[T_{\zeta} \cap F\right.$ é infinito ]

A construção é por recursão em $\zeta<\kappa$. Seja então $0 \leqslant \zeta<\kappa$ e suponhamos $\mathcal{T}=\left\{T_{\xi}: \xi<\zeta\right\}$ bem construída. Fixemos $\delta<\kappa$; afirmamos que $\mathcal{F}_{\delta} \cup \mathcal{F}_{\zeta} \cup \mathcal{T}$ possui a s.f.i.p. Isso é imediato, pois $\mathcal{F}$ é fechado para intersecções finitas, (14) faz com que $\mathcal{T}$ seja "=*-fechado" para intersecções finitas e (15) resolve a questão. Assim, de $\left|\mathcal{F}_{\delta} \cup \mathcal{F}_{\zeta} \cup \mathcal{T}\right|<\mathfrak{p}$ podemos tomar uma pseudo-intersecção infinita $P_{\delta}$ de $\mathcal{F}_{\delta} \cup \mathcal{F}_{\zeta} \cup \mathcal{T}$. Considere agora $\mathcal{A}=\mathcal{F}_{\zeta} \cup \mathcal{T}$ e $\mathcal{B}=\left\{P_{\delta}: \delta<\kappa\right\}$ e aplique o Fato 2.2 ; existe então uma pseudointersecção infinita $T_{\zeta}$ de $\mathcal{F}_{\zeta} \cup \mathcal{T}$ satisfazendo $(\forall \delta<\kappa)\left[T_{\zeta} \cap P_{\delta}\right.$ é infinito ]. É evidente que (13) e (14) valem para $T_{\zeta}$. Para verificar (15), note que $(\forall F \in \mathcal{F})(\exists \delta<\kappa)\left[F \in \mathcal{F}_{\delta}\right]$, donde $(\forall F \in \mathcal{F})(\exists \delta<\kappa)\left[P_{\delta} \subseteq{ }^{*} F\right]$ e portanto $T_{\zeta} \cap F$ é infinito pois $T_{\zeta} \cap P_{\delta}$ é infinito. Assim, a construção está justificada e a família $\left\{T_{\zeta}: \zeta<\kappa\right\}$ bem construída. $\left\{T_{\zeta}: \zeta<\kappa\right\}$ possui a s.f.i.p., por (14), e $\kappa<\mathfrak{p}$ nos fornece uma pseudo-interseç̧ão infinita $T$ de $\left\{T_{\zeta}: \zeta<\kappa\right\}$ e $T$ é pseudo-intersecção infinita de $\mathcal{F}$ por (13).

A regularidade de $\mathfrak{p}$ sugere tratar da questão dos $P$-pontos e compreender o motivo da escolha da letra " $\mathfrak{p}$ ". Um ultrafiltro livre $u$ é um $P$-ponto em $\beta \omega \backslash \omega=\omega^{*}$ se a intersecção de qualquer família enumerável de vizinhanças de $u$ é ainda uma vizinhança de $u$. No artigo [Rud56], W.Rudin apresentou uma demonstração de que $C H$ implica na existência de $P$-pontos; na verdade, $C H$ pode ser enfraquecida para " $\mathfrak{p}=\mathfrak{c}$ ".

Proposição 2.1 Se $\mathfrak{p}=\mathfrak{c}$, existem P-pontos $\mathrm{em} \omega^{*}$.

Demonstração Sabemos que todos os clopens de $\omega^{*}$ são da forma $W(E)$ para algum $E \in[\omega]^{\omega}$ (ver capítulo 1). Assim, "p $=\mathfrak{c}$ " implica na existência de uma enumeração $\left\{W_{\alpha}: \alpha<\mathfrak{p}\right\}$ de todos os clopens de $\omega^{*}$, com $W_{0}=\omega^{*}$. Construiremos agora uma família decrescente $\left\{A_{\alpha}: \alpha<\mathfrak{p}\right\}$ de clopens não-vazios de $\omega^{*}$ e uma família de clopens não-vazios 
$\left\{C_{\alpha}: 1 \leqslant \alpha<\mathfrak{c}\right\}$ satisfazendo $A_{0}=W_{0}=\omega^{*}$ e, para todo $1 \leqslant \alpha<\mathfrak{p}$

(1) $C_{\alpha} \subseteq \bigcap_{\beta<\alpha} A_{\beta}$

(2) $A_{\alpha}=C_{\alpha}$ se $C_{\alpha} \cap W_{\alpha}=\emptyset$ e $A_{\alpha}=C_{\alpha} \cap W_{\alpha}$ se $C_{\alpha} \cap W_{\alpha} \neq \emptyset$

(Note que, da condição (2), teremos para todo $\alpha$ que $A_{\alpha}$ ou é disjunto ou está contido em $W_{\alpha}$.)

Justifiquemos a construção: seja $1 \leqslant \alpha<\mathfrak{p}$ e suponha que $\left\{A_{\beta}: \beta<\alpha\right\}$ esteja bem construída e seja decrescente. Então $\bigcap_{\beta<\alpha} A_{\beta}$ é uma intersecção de menos do que $\mathfrak{p}$ clopens que satisfazem a p.i.f. em $\omega^{*}$. Trabalhando com os subconjuntos infinitos de $\omega$ que estão naturalmente associados a cada um dos $A_{\beta}$ - que são no máximo $|\alpha|<\mathfrak{p}$ e satisfazem a s.f.i.p. - , é fácil encontrar um clopen não-vazio $C_{\alpha}$ que esteja contido em $\bigcap_{\beta<\alpha} A_{\beta}$. (1) fica então satisfeita e (2) define $A_{\alpha}$ conforme desejado.

Considere agora $A=\bigcap_{\alpha<\mathrm{p}} A_{\alpha} ; A$ é uma intersecção de uma família de fechados com a p.i.f. em $\omega^{*}$, daí $A \neq \emptyset$. Por outro lado, se $A \cap W_{\beta} \neq \emptyset$ para algum $\beta<\mathfrak{p}$, tem-se $A \subseteq A_{\beta} \subseteq W_{\beta}$, e, como $\left\{W_{\alpha}: \alpha<\mathfrak{p}\right\}$ é uma base para $\omega^{*}$, temos que a única chance para $A$ é ser um conjunto unitário, i.e., $A=\{u\}$ para algum $u \in \omega^{*}$. É fácil ver também que $\left\{A_{\alpha}: \alpha<\mathfrak{p}\right\}$ é uma base local para $u$. Afirmamos que $u$ é um $P$-ponto de $\omega^{*}$. De fato: seja $\left\{G_{n}: n<\omega\right\}$ uma família de vizinhanças de $u$. Para cada $n<\omega$ existe $\alpha_{n}<\mathfrak{p}$ tal que $u \in A_{\alpha_{n}} \subseteq G_{n}$. Considerando-se $\alpha=\sup \left\{\alpha_{n}: n<\omega\right\}$, tem-se $\alpha<\mathfrak{p}$ (segue da regularidade de $\mathfrak{p}$ ou de $\left.\omega<c f\left(2^{\omega}\right)\right)$ e $u \in A_{\alpha+1} \subseteq \bigcap_{n<\omega} G_{n}$ e $\bigcap_{n<\omega} G_{n}$ é vizinhança de $u$.

\subsection{Definições alternativas para os pequenos cardinais}

Nesta seção, daremos definições alternativas para nossos pequenos cardinais, isto é, exibiremos enunciados conjuntísticos que definem de outro modo os cardinais que estamos estudando. Em algumas situações, mais do que uma possível formulação alternativa, esses enunciados evidenciam importantes caracterizações e propriedades desses cardinais, propriedades essas que serão úteis em nosso trabalho (ou que já foram úteis, como o 
resultado utilizado para o Fato 2.2).

Trataremos com mais detalhe dos cardinais $\mathfrak{a}, \mathfrak{b}, \mathfrak{d}, \mathfrak{p}$ e $\mathfrak{t}$. Quanto ao cardinal $\mathfrak{s}, \mathrm{o}$ mesmo será caracterizado topologicamente no Capítulo 4; antes, porém, podemos fazer uma pequena variação do argumento utilizado na demonstração de $\mathfrak{s} \leqslant \mathfrak{d}$ e temos o

Fato $2.3 \quad \mathfrak{s}=\mathfrak{s}^{\prime}$, onde

$$
\begin{aligned}
& \mathfrak{s}^{\prime}=\min \left\{|F|: F \subseteq{ }^{\omega} \omega,(\forall f \in F)(\forall n<\omega)\left[f^{n}(0)<f^{n+1}(0)\right],\left\{S_{f}: f \in F\right\}\right. \text { é } \\
& \qquad \text { família splitting }\}
\end{aligned}
$$

sendo $f^{n}$ e $S_{f}$ definidos como na demonstração de $\mathfrak{s} \leqslant \mathfrak{0}$.

Demonstração A definição de $\mathfrak{s}^{\prime}$ nos dá $\mathfrak{s} \leqslant \mathfrak{s}^{\prime}$. Para a desigualdade contrária, tome $\left\{S_{\alpha}: \alpha<\mathfrak{s}\right\}$ uma família splitting. Podemos supor s.p.g. que $(\forall \alpha<\mathfrak{s})\left[\left|S_{\alpha}\right|=\left|\omega \backslash S_{\alpha}\right|=\right.$ $\omega$ ]. Então, para cada $\alpha<\mathfrak{s}$, definimos $f_{\alpha} \in{ }^{\omega} \omega$ de modo que $S_{f_{\alpha}}={ }^{*} S_{\alpha}$ (o que é fácil e faz com que $f_{\alpha}$ satisfaça $\left.(\forall n<\omega)(\forall \alpha<\mathfrak{s})\left[f_{\alpha}^{n}(0)<f_{\alpha}^{n+1}(0)\right]\right)$ e tenho $F=\left\{f_{\alpha}: \alpha<\mathfrak{s}\right\}$ satisfazendo a definição de $\mathfrak{s}^{\prime}$, daí $\mathfrak{s}^{\prime} \leqslant \mathfrak{s}$.

Tratemos agora do cardinal $\mathfrak{a}$.

Fato $2.4 \quad \mathfrak{a}=\mathfrak{a}_{1}=\mathfrak{a}_{2}$, onde

$$
\begin{aligned}
& \mathfrak{a}_{1}=\min \left\{|\mathcal{A}|: \mathcal{A} \subseteq[\omega]^{\omega} \text { e existe uma partição infinita } \mathcal{D} \text { de } \omega \text { tal que } \mathcal{D} \cap \mathcal{A}=\emptyset\right. \\
& \quad e \mathcal{D} \cup \mathcal{A} \text { é uma família m.a.d. infinita em } \omega\} \\
& \mathfrak{a}_{2}=\min \left\{|\mathcal{A}|: \mathcal{A} \subseteq[\omega \times \omega]^{\omega},(\forall k<\omega)[\{k\} \times \omega \notin \mathcal{A}] \text { e } \mathcal{A} \cup\{\{k\} \times \omega: k<\omega\}\right. \text { é } \\
& \text { uma família m.a.d. em } \omega \times \omega\}
\end{aligned}
$$

Demonstração A igualdade $\mathfrak{a}_{1}=\mathfrak{a}_{2}$ é clara: para $\mathfrak{a}_{1} \leqslant \mathfrak{a}_{2}$, qualquer bijeção entre $\omega$ e $\omega \times \omega$ é suficiente, e para $\mathfrak{a}_{2} \leqslant \mathfrak{a}_{1}$ escolhemos uma bijeção que leva cada elemento da partição de $\omega$ em questão numa "linha vertical" de $\omega \times \omega$. Note que partições infinitas de $\omega$ são enumeráveis e que $\mathcal{D}$ satisfazendo a definição de $\mathfrak{a}_{1}$ satisfaz $\mathcal{D} \subseteq[\omega]^{\omega}$.

De $\omega_{1} \leqslant \mathfrak{a} \leqslant \mathfrak{a}_{1}+\omega$, vem $\mathfrak{a} \leqslant \mathfrak{a}_{1} \operatorname{logo}$ o que deve ser feito é $\mathfrak{a}_{1} \leqslant \mathfrak{a}$,e o argumento a ser utilizado para tal é uma pequena adaptação do que foi feito para "a parte enumerável" da família a.d. infinita usada na demonstração de $\mathfrak{b} \leqslant \mathfrak{a}$. De fato, seja $\mathcal{B}$ uma família 
m.a.d. infinita, com $|\mathcal{B}|=\mathfrak{a}$, e seja $\left\{A_{n}: n<\omega\right\}$ uma subfamília enumerável infinita de B. Construo então $\left\{A_{n}^{\prime}: n<\omega\right\} \subseteq[\omega]^{\omega}$ pondo $A_{n}^{\prime}=\left(\{n\} \cup A_{n}\right) \backslash \bigcup_{k<n}\left(A_{k} \cup\{k\}\right)$ para todo $n<\omega$. É imediato que $\left\{A_{n}^{\prime}: n<\omega\right\}$ é uma partição de $\omega$ em conjuntos infinitos disjuntos e que $\left(\forall X \in[\omega]^{\omega}\right)(\forall n<\omega)\left[\left(\left|X \cap A_{n}\right|=\omega\right) \Leftrightarrow\left(\left|X \cap A_{n}^{\prime}\right|=\omega\right)\right]$. Desse modo, $\mathcal{A}=\mathcal{B} \backslash\left\{A_{n}: n<\omega\right\}$ e $\mathcal{D}=\left\{A_{n}^{\prime}: n<\omega\right\}$ nos dão $\mathfrak{a}_{1} \leqslant \mathfrak{a}$.

A terminologia que definiremos agora, necessária para o enunciado do próximo teorema, será de vital importância para este trabalho. No capítulo seguinte, quando trataremos de gaps em conjuntos parcialmente ordenados, veremos que determinados pares de subfamílias de $[\omega]^{\omega}$ que são bem ordenadas por $C^{*}$ e que satisfazem a primeira parte da próxima definição e não satisfazem a segunda são, de certa forma, gaps.

Definição 2.2 Sejam $\mathcal{F}$ e $\mathcal{G}$ famílias de conjuntos enumeráveis infinitos.

i) Dizemos que $\mathcal{F}$ e $\mathcal{G}$ são ortogonais, e indicamos isso por $\mathcal{F} \perp \mathcal{G}$, se

$$
(\forall F \in \mathcal{F})(\forall G \in \mathcal{G})[|F \cap G|<\omega]
$$

ii) Dizemos que $\mathcal{F}$ e $\mathcal{G}$ podem ser separados se existir um conjunto $S$ tal que

$$
(\forall F \in \mathcal{F})\left[F \subseteq^{*} S^{\prime}\right] \wedge(\forall G \in \mathcal{G})[|G \cap S|<\omega]
$$

Observamos que, se $S$ é um conjunto que separa $\mathcal{F}$ e $\mathcal{G}$, então $S^{\prime}=\bigcup(\mathcal{F} \cup \mathcal{G}) \backslash S$ separa $\mathcal{G}$ e $\mathcal{F}$ (basta notar que, para quaisquer $G \in \mathcal{G}$ e $F \in \mathcal{F}, G \backslash S^{\prime} \subseteq G \cap S^{\prime}$ e $F \cap S^{\prime} \subseteq F \backslash S$ ), logo a relação "podem ser separados" é simétrica. Escreveremos $F \perp \mathcal{G}$ se $\{F\} \perp \mathcal{G}$. Usando álgebra elementar de conjuntos, podemos verificar que, se duas famílias podem ser separadas, então elas são ortogonais; porém, veremos que a recíproca nao é verdadeira, e, como já demos a entender no parágrafo anterior à definição 2.2 , teremos grande interesse em trabalhar com famílias ortogonais e que não podem ser separadas.

Definição 2.3 Se $B \subseteq{ }^{\omega} \omega$ e $I \in[\omega]^{\omega}$, dizemos que $B$ é ilimitado em $I$ se

$$
\left(\forall f \in{ }^{\omega} \omega\right)(\exists g \in B)[|\{n \in I: f(n)<g(n)\}|=\omega]
$$


Veja que a condição definida acima, " $B$ ser ilimitado em $I$ ", corresponde exatamente à situação em que $\left\{g\lceil I: g \in B\}\right.$ é ilimitado em $\left\langle{ }^{I} \omega, \leqslant^{*}\right\rangle$.

Finalmente, apresentamos o

Teorema 2.2 Seja $\mathcal{V}$ a coleção de "linhas verticais" em $\omega \times \omega$, isto é, $\mathcal{V}=\{\{k\} \times \omega$ : $k<\omega\}$.

$$
\begin{aligned}
& \text { Então } \mathfrak{b}=\mathfrak{b}_{i} \text { para } i \in\{1, \ldots, 7\} \text {, onde } \\
& \mathfrak{b}_{1}=\min \left\{|B|: B \text { é um subconjunto ilimitado de }{ }^{\omega} \omega\right. \text { formado por funções estrita- } \\
& \text { mente crescentes e que é bem-ordenado por } \left.<^{*}\right\} \\
& \mathfrak{b}_{2}=\min \left\{|B|: B \subseteq{ }^{\omega} \omega \text { e } B \text { é ilimitado em qualquer subconjunto infinito de } \omega\right\} \\
& \mathfrak{b}_{3}=\min \left\{|\mathcal{B}|: \mathcal{B} \subseteq[\omega \times \omega]^{\omega} \text { é bem-ordenado por } \subset^{*}, \mathcal{B} \perp \mathcal{V} \text { e }\left(\forall X \in[\omega \times \omega]^{\omega}\right)\right. \\
& [X \perp \mathcal{V} \Rightarrow \exists B \in \mathcal{B}[|X \cap B|=\omega]]\} \\
& \mathfrak{b}_{4} \text { : como em } \mathfrak{b}_{3} \text {, mas sem "é bem-ordenado por } \subset^{*} \text { ". } \\
& \mathfrak{b}_{5}=\min \left\{|\mathcal{B}|: \mathcal{B} \subseteq[\omega]^{\omega} \text { e } \exists \mathcal{C} \subseteq[\omega]^{\omega}[|\mathcal{C}|=\omega, \mathcal{B} \cap \mathcal{C}=\emptyset, \mathcal{B} \cup \mathcal{C} \text { é família a.d. e }\right. \\
& \left.\left.\left(\forall D \in[\mathcal{C}]^{\omega}\right)[\mathcal{B} \text { e } \mathcal{D} \text { não podem ser separados }]\right]\right\} \\
& \mathfrak{b}_{6}=\min \left\{|\mathcal{B}|: \mathcal{B} \subseteq[\omega]^{\omega} \text { e } \exists \mathcal{C} \subseteq[\omega]^{\omega}[|\mathcal{C}|=\omega, \mathcal{B} \perp \mathcal{C}, \mathcal{B} \text { e } \mathcal{C} \text { são bem ordenados }\right. \\
& \text { por } \subseteq \text { * e } \mathcal{B} \text { e } \mathcal{C} \text { não podem ser separados }]\}
\end{aligned}
$$

$\mathfrak{b}_{7}$ : como em $\mathfrak{b}_{6}$, mas sem "B e $\mathcal{C}$ são bem-ordenados por $\subseteq^{*}$ ".

Demonstração A igualdade $\mathfrak{b}=\mathfrak{b}_{1}$ foi estabelecida na prova do item $d$ ) do Teorema 2.1. Já $\mathfrak{b} \leqslant \mathfrak{b}_{2}, \mathfrak{b}_{4} \leqslant \mathfrak{b}_{3}$ e $\mathfrak{b}_{7} \leqslant \mathfrak{b}_{6}$ são óbvias. Mostraremos que $\mathfrak{b}_{1} \geqslant \mathfrak{b}_{2}, \mathfrak{b}_{1} \geqslant \mathfrak{b}_{3} \geqslant \mathfrak{b}_{6}$, $\mathfrak{b}_{1} \geqslant \mathfrak{b}_{5} \geqslant \mathfrak{b}_{7}$ e $\mathfrak{b}_{4} \geqslant \mathfrak{b}_{7} \geqslant \mathfrak{b}$, o que completará a demonstração.

Antes de iniciarmos a demonstração das desigualdades anunciadas, definiremos uma notação específica para certos conjuntos. Para $f \in{ }^{\omega} \omega$, definimos $L_{f}=\{\langle k, n\rangle \in$ $\omega \times \omega: n \leqslant f(k)\}$ e observamos que

(1) $\left(\forall f, g \in{ }^{\omega} \omega\right)\left[f<^{*} g \Longleftrightarrow L_{f} \subset^{*} L_{g}\right]$

Para $X \in[\omega \times \omega]^{\omega}$, definimos $K_{X}=\{k<\omega: X \cap(\{k\} \times \omega) \neq \emptyset\}$ e se $X \perp \mathcal{V}$ 
definimos $f_{X} \in{ }^{\omega} \omega$ pondo, para cada $k<\omega, f_{X}(k)=\max \{n<\omega: n=0$ ou $\langle k, n\rangle \in X\}$. Note que, nessas condições,

$$
\text { (2) } X \subseteq L_{f_{X}} \cap\left(K_{X} \times \omega\right)
$$

Prova de $\mathfrak{b}_{2} \leqslant \mathfrak{b}_{1}$ : É suficiente mostrar o seguinte

Fato 2.5 Se $B \subseteq{ }^{\omega} \omega$ é ilimitado e formado por funções não-decrescentes, então $B$ é ilimitado em qualquer subconjunto infinito de $\omega$.

Prova do Fato: Sejam $f \in{ }^{\omega} \omega$ e $I \in[\omega]^{\omega}$ quaisquer; mostraremos que existe $g \in B$ tal que $\{i \in I: f(i)<g(i)\}$ é infinito. Definimos $\hat{f} \in{ }^{\omega} \omega$ pondo

$$
\hat{f}(k)=\max \{f(n): n \leqslant \min \{i \in I: k \leqslant i\}\}
$$

Como B é ilimitado, existe $g \in B$ tal que $g \mathbb{*}^{*} \hat{f}$, isto é, $J=\{j<\omega: \hat{f}(j)<g(j)\}$ é infinito. Agora, para cada $j \in J$, se $i_{j}=\min \{k \in I: j \leqslant k\}$, então $g\left(i_{j}\right) \geqslant g(j)>$ $\hat{f}(j) \geqslant f\left(i_{j}\right)$ (pois $g$ é não decrescente, $j \in J$ e $\hat{f}(j)=\max \left\{f(n): n \leqslant i_{j}\right\}$ ). Como é evidente que o conjunto $\left\{i_{j}: j \in J\right\}$ não pode ser finito, conclui-se que $\{i \in I: f(i)<g(i)\}$ é infinito.

Prova de $\mathfrak{b}_{3} \leqslant \mathfrak{b}_{1}$ : Tome $B$ como na definição de $\mathfrak{b}_{1}$ e de tamanho mínimo; afirmamos que, se tomarmos $\mathcal{B}=\left\{L_{f}: f \in B\right\}$, então $\mathcal{B}$ satisfaz as condições da definição de $\mathfrak{b}_{3}$, donde vem $\mathfrak{b}_{3} \leqslant|\mathcal{B}|=\mathfrak{b}_{1}$.

De fato: (1) mostra que $\mathcal{B}$ está bem-ordenado por $\subset^{*}$, e obviamente $\mathcal{B} \perp \mathcal{V}$ (já que $L_{f} \perp \mathcal{V}$ para qualquer $\left.f \in{ }^{\omega} \omega\right)$. Seja então $X \in[\omega \times \omega]^{\omega}$ tal que $X \perp \mathcal{V}$. Claramente $K_{X}$ é infinito, e o Fato 2.5 nos dá $B$ ilimitado em $K_{X}$ donde existe $g \in B$ tal que $J=\left\{k \in K_{X}: f_{X}(k) \leqslant g(k)\right\}$ é infinito. Segue que $f_{X} \uparrow J=\left\{\left\langle k, f_{X}(k)\right\rangle: k \in J\right\}$ é um subconjunto infinito tanto de $X$ como de $L_{g}$.

Prova de $\mathfrak{b}_{5} \leqslant \mathfrak{b}_{1}$ : Seja $\mathfrak{b}_{5}^{\prime}$ definido substituindo-se "[$[\omega] \omega "$ por " $[\omega \times \omega]$ " na definição de $\mathfrak{b}_{5}$; de $\mathfrak{b}_{5}=\mathfrak{b}_{5}^{\prime}$ é suficiente mostrarmos que $\mathfrak{b}_{5}^{\prime} \leqslant \mathfrak{b}_{1}$. Para isso, tomaremos $B$ como na definição de $\mathfrak{b}_{1}$ e de tamanho mínimo e mostraremos que $\mathcal{B}=B$ e $\mathcal{C}=\mathcal{V}$ satisfazem a definição de $\mathfrak{b}_{5}^{\prime}$, donde $\mathfrak{b}_{5}^{\prime} \leqslant|\mathcal{B}|=\mathfrak{b}_{1}$.

De fato: seja $B \subseteq \omega_{\omega}$ ilimitado, com $|B|=\mathfrak{b}_{1}, B$ bem-ordenado por $<^{*}$ e formado por funções estritamente crescentes; podemos supor também, s.p.g., que $B$ enquanto 
subfamília de $[\omega \times \omega]^{\omega}$ é a.d., conforme Observação 2.1. Daí, fazendo $\mathcal{B}=B$ e $\mathcal{C}=\mathcal{V}$, temos $|\mathcal{C}|=\omega, \mathcal{B} \cap \mathcal{C}=\emptyset$ e $\mathcal{B} \cup \mathcal{C}$ a.d. Considere agora $\mathcal{D} \in[\mathcal{C}]^{\omega}$ qualquer e um conjunto $S \in[\omega \times \omega]^{\omega}$ tal que $(\forall C \in \mathcal{D})\left[C \subseteq \subseteq^{*}\right]$; mostraremos que existe $g \in B$ tal que $g \cap S$ é infinito, donde $\mathcal{B}$ e $\mathcal{D}$ não podem ser separados. Seja então $D=\{k<\omega:\{k\} \times \omega \in \mathcal{D}\} ; D$ é infinito. Defino $f \in{ }^{\omega} \omega$ pondo, para cada $k<\omega, f(k)=\max \{n<\omega: n=0$ ou $(n \geqslant 1$ $\mathrm{e}\langle k, n-1\rangle \notin S)\}$. É evidente que tal $f$ satisfaz $(\forall k, n<\omega)[n \geqslant f(k) \Rightarrow\langle k, n\rangle \in S]$. Pelo Fato $2.5, B$ é ilimitado em $D$, donde existe $g \in B$ tal que $I=\{k \in D: f(k) \leqslant g(k)\}$ é infinito. Segue que $g\lceil I \subseteq S$, donde $g \cap S$ é infinito.

Prova de $\mathfrak{b}_{6} \leqslant \mathfrak{b}_{3}$ : Definimos $\mathfrak{b}_{6}^{\prime}$ como $\mathfrak{b}_{6}$, substituindo " $[\omega] \omega "$ por " $[\omega \times \omega]$ " e mostraremos que $\mathfrak{b}_{6}^{\prime} \leqslant \mathfrak{b}_{3}$. Seja $\mathcal{B} \subseteq[\omega \times \omega]^{\omega}$ nas condições da definição de $\mathfrak{b}_{3} \operatorname{com}|\mathcal{B}|=\mathfrak{b}_{3}$, e seja $\mathcal{C}=\{k \times \omega: 1 \leqslant k<\omega\}=\{\{0, \ldots, k-1\} \times \omega: 1 \leqslant k<\omega\}$. Então $|\mathcal{C}|=\omega, \mathcal{B} \perp \mathcal{V}$ implica $\mathcal{B} \perp \mathcal{C}$ e $\mathcal{B}$ e $\mathcal{C}$ estão bem-ordenados por $\subseteq^{*}$ (pois $\mathcal{B}$ está bem-ordenado por $\subset^{*}$ e $\mathcal{C}$ está bem-ordenado por $\subseteq$ ). Seja então $S \in[\omega \times \omega]^{\omega}$ tal que $(\forall C \in \mathcal{C})\left[C \subseteq \subseteq^{*} S\right]$. Tem-se $S \cap(\{k\} \times \omega) \neq \emptyset$ para todo $k<\omega$ portanto posso definir $f \in{ }^{\omega} \omega$ pondo, para cada $k<\omega$, $f(k)=\min \{n<\omega:\langle k, n\rangle \in S\}$. Como $f \perp \mathcal{V}$, existe $B \in \mathcal{B}$ tal que $|B \cap f|=\omega$. Daí, $f \subseteq S$ implica $|B \cap S|=\omega$.

Prova de $\mathfrak{b}_{7} \leqslant \mathfrak{b}_{4}$ : Idêntica à prova de $\mathfrak{b}_{6} \leqslant \mathfrak{b}_{3}$, mas sem " $\mathcal{B}$ e $\mathcal{C}$ estão bem-ordenados por $\subseteq * "$.

Prova de $\mathfrak{b}_{7} \leqslant \mathfrak{b}_{5}: \operatorname{Se} \mathcal{B}, \mathcal{C} \subseteq[\omega]^{\omega}$ são tais que $\mathcal{B} \cup \mathcal{C}$ é família a.d., então $\mathcal{B} \perp \mathcal{C}$.

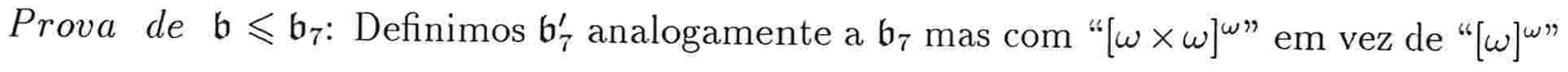
e a partir daí tomamos $\mathcal{B}, \mathcal{C} \subseteq[\omega \times \omega]^{\omega}$ tais que $|\mathcal{B}|=\mathfrak{b}_{7}^{\prime},|\mathcal{C}|=\omega, \mathcal{B} \perp \mathcal{C}$ e $\mathcal{B}$ e $\mathcal{C}$ não podem ser separados; construiremos então $B \subseteq \omega_{\omega}$ ilimitado com $|B| \leqslant|\mathcal{B}|=\mathfrak{b}_{7}^{\prime}$ e daí teremos $\mathfrak{b} \leqslant \mathfrak{b}_{7}^{\prime}=\mathfrak{b}_{7}$.

De fato: $\operatorname{sejam~} \mathcal{B}$ e $\mathcal{C}$ como no parágrafo anterior. Observamos inicialmente que, se $\left\{C_{1}, \ldots, C_{k}\right\}$ é um subconjunto finito de $\mathcal{C}$, então, de $(\forall B \in \mathcal{B})\left[\left|B \cap\left(\bigcup_{i=1}^{k} C_{i}\right)\right|<\omega\right]$ e de $\mathcal{C}$ e $\mathcal{B}$ não poderem ser separados, existe algum $C \in \mathcal{C}$ tal que $C \backslash\left(\bigcup_{i=1}^{k} C_{i}\right)$ é infinito. Seja então $\mathcal{C}=\left\{C_{n}: n<\omega\right\}$. Com base no que foi observado, é possível construir recursivamente 
uma subfamília $\left\{C_{k_{n}}: n<\omega\right\} \subseteq \mathcal{C}$ satisfazendo as afirmações

$$
\begin{aligned}
& C_{k_{0}} \backslash C_{0} \text { é infinito; } \\
& C_{k_{1}} \backslash\left(C_{k_{0}} \cup C_{0} \cup C_{1}\right) \text { é infinito; } \\
& C_{k_{2}} \backslash\left(C_{k_{1}} \cup C_{k_{0}} \cup C_{0} \cup C_{1} \cup C_{2}\right) \text { é infinito; }
\end{aligned}
$$

ou, mais geralmente, $C_{k_{n}} \backslash\left(\bigcup_{m<n} C_{k_{m}} \cup \bigcup_{m \leqslant n} C_{m}\right)$ é infinito para todo $n<\omega$. Decompomos agora

$$
(\omega \times \omega) \backslash \bigcup \mathcal{C}=\bigcup_{i<\omega} A_{i}
$$

onde $\left\{A_{i}: i<\omega\right\} \subseteq[\omega \times \omega]^{<\omega}$ é disjunta (isto é, os $A_{i}$ 's são subconjuntos disjuntos de $\omega \times \omega$ e cada um deles é finito (eventualmente, vazio)). Definimos agora

$$
\begin{aligned}
& X_{0}=C_{0} \cup A_{0} ; \\
& X_{1}=\left(\left(C_{k_{0}} \cup C_{1}\right) \backslash X_{0}\right) \cup A_{1} ; \\
& X_{2}=\left(\left(C_{k_{1}} \cup C_{2}\right) \backslash\left(X_{0} \cup X_{1}\right)\right) \cup A_{2} ; \\
& \vdots \\
& X_{i}=\left(\left(C_{k_{i-1}} \cup C_{i}\right) \backslash\left(\bigcup_{j<i} X_{j}\right)\right) \cup A_{i} \text { para cada } i \geqslant 1 .
\end{aligned}
$$

Segue que $\left\{X_{i}: i<\omega\right\} \subseteq[\omega \times \omega]^{\omega},\left\{X_{i}: i<\omega\right\}$ é família disjunta e $\bigcup_{i<\omega} X_{i}=\omega \times \omega$.

Seja então $h$ uma permutação de $\omega \times \omega$ que leva (bijetivamente) ${ }_{i} X_{i}$ em $\{i\} \times \omega$ e identifiquemos $\mathcal{B}$ e $\mathcal{C}$ com suas respectivas "imagens" pela permutação $h$. Podemos perceber então que, sem nenhuma perda de generalidade, podemos supor $\mathcal{B} \perp \mathcal{V}$ e que $\mathcal{B}$ e $\mathcal{V}$ não podem ser separados (de fato: cada "linha vertical" de $\omega \times \omega$, na construção anterior, pode ser decomposta (a menos de $=^{*}$ ) como uma união finita de subconjuntos de elementos de $\mathcal{C}$, donde $(\forall B \in \mathcal{B})(\forall k<\omega)[|B \cap(\{k\} \times \omega)|<\omega]$. Também é imediato, dentro das suposições feitas, que para todo $i<\omega$ o elemento $C_{i}$ está inteiramente contido em $(i+1) \times \omega$, e daí é fácil concluir que $\mathcal{B}$ e $\mathcal{V}$ não podem ser separados. Observamos que tudo o que fizemos até agora - a construção dos $X_{i}$ 's e da permutação $h$ - foi mostrar que, dados $\mathcal{B}$ e $\mathcal{C}$ satisfazendo as condições da hipótese, $\mathcal{B}$ e $\mathcal{C}$ podem ser convenientemente rearranjados de modo que valham as mesmas condições para $\mathcal{B}$ e $\mathcal{V}$ ). Assim, $f_{X}$ pode ser definida para todo $X \in \mathcal{B}$. Sejam então $B=\left\{f_{X}: X \in \mathcal{B}\right\}$ e $\mathcal{B}^{\prime}=\left\{L_{f}: f \in B\right\}$. Temos 
$\mathcal{B}^{\prime} \perp \mathcal{V}$, e (2) nos dá que $\mathcal{B}^{\prime}$ e $\mathcal{V}$ não podem ser separados (basta notar que, se $L_{f_{X}} \subseteq^{*} S$ então $X \subseteq^{*} S$, para $X, S \in[\omega \times \omega]^{\omega}$ quaisquer).

Afirmamos agora que $B$ é ilimitado. De fato: seja $f \in{ }^{\omega} \omega$ qualquer. Então $L_{f} \perp \mathcal{V}$. Como $\mathcal{B}^{\prime}$ e $\mathcal{V}$ não podem ser separados, existe $g \in B$ tal que $L_{g} \nsubseteq^{*} L_{f}$. Daí, (1) nos dá que $g \mathbb{K}^{*} f$ e portanto $f$ não é majorante de $B$. Segue $\mathfrak{b} \leqslant|B|=|\mathcal{B}|=\mathfrak{b}_{7}^{\prime}=\mathfrak{b}_{7}$, conforme desejado.

Observação 2.2 Convidamos o leitor a, após rever as demonstrações de $\mathfrak{b}_{7} \leqslant \mathfrak{b}_{5}$ e $\mathfrak{b}_{5} \leqslant$ $\mathfrak{b}_{1}$, concluir que vale também

$$
\begin{aligned}
& \mathfrak{b}=\min \left\{|\mathcal{B}|: \mathcal{B} \subseteq[\omega]^{\omega} \text { e } \exists \mathcal{C} \in[\omega]^{\omega}[|\mathcal{C}|=\omega, \mathcal{B} \cap \mathcal{C}=\emptyset, \mathcal{B} \cup \mathcal{C} \text { é a.d. e } \mathcal{B} \text { e } \mathcal{C} \text { não }\right. \\
& \text { podem ser separados }]\}
\end{aligned}
$$

Tratemos agora do cardinal $\mathfrak{d}$. Antes, vamos estabelecer mais uma relação de ordem em ${ }^{\omega} \omega$ : dadas $f$ e $g$ em ${ }^{\omega} \omega$, diremos que $f \leqslant g$ se e só se $f(n) \leqslant g(n)$ para todos os números naturais $n$ (lembrando que $f \leqslant *^{*} g$ se e só se $f(n) \leqslant g(n)$ para todos exceto no máximo finitos números naturais $n$ ). Definimos agora também o conceito de "ser cofinal numa família”.

Definição 2.4 Dizemos que $D \subseteq{ }^{\omega} \omega$ é cofinal em $\mathcal{A} \subseteq[\omega]^{\omega}$ se

$$
\left(\forall f \in{ }^{\omega} \omega\right)(\exists g \in D)(\exists A \in \mathcal{A})[f \leqslant g \text { em A, i.e., }(\forall a \in A)[f(a) \leqslant g(a)]] .
$$

Tendo definido $\leqslant$, pode-se falar agora em subconjuntos cofinais na estrutura $\left\langle{ }^{\omega} \omega\right.$, $\leqslant\rangle ; D \subseteq{ }^{\omega} \omega$ é cofinal em $\left\langle{ }^{\omega} \omega, \leqslant\right\rangle$ se e só se $\left(\forall f \in{ }^{\omega} \omega\right)(\exists g \in D)(\forall n<\omega)[f(n) \leqslant g(n)]$. Assim, segundo a definição que acabamos de apresentar, $D$ é cofinal em $\left\langle{ }^{\omega} \omega, \leqslant\right\rangle$ se e só se $D$ é cofinal em $\{\omega\}$.

Teorema $2.3 \quad \mathfrak{d}=\mathfrak{d}_{1}=\mathfrak{d}_{2}$, onde

$$
\begin{aligned}
& \mathfrak{d}_{1}=\min \left\{|D|: D \text { é cofinal em }\left\langle{ }^{\omega} \omega, \leqslant\right\rangle\right\} \\
& \mathfrak{d}_{2}=\min \left\{|D|+|\mathcal{A}|: D \subseteq{ }^{\omega} \omega, \mathcal{A} \subseteq[\omega]^{\omega} \text { e } D \text { é cofinal em } \mathcal{A}\right\}
\end{aligned}
$$


Demonstração Mostraremos que $\mathfrak{d} \leqslant \mathfrak{d}_{1} \leqslant \mathfrak{d}_{2} \leqslant \mathfrak{o}$.

Prova de $\mathfrak{d} \leqslant \mathfrak{o}_{1}$ : Se $D$ é cofinal em $\left\langle{ }^{\omega} \omega, \leqslant\right\rangle$, em particular é cofinal em $\left\langle{ }^{\omega} \omega, \leqslant^{*}\right\rangle$.

Prova de $\mathfrak{d}_{2} \leqslant \mathfrak{o}$ : Tome $D \subseteq \omega_{\omega}$ dominante com $|D|=\mathfrak{o}$. É imediato que $D$ é cofinal em $\mathcal{A}=\{\omega \backslash A: A \subseteq \omega$ é finito $\}$, donde $\mathfrak{d}_{2} \leqslant|D|+|\mathcal{A}|=\mathfrak{d}+\omega=\mathfrak{d}$.

Prova de $\mathfrak{d}_{1} \leqslant \mathfrak{d}_{2}$ : Seja $D \subseteq{ }^{\omega} \omega$ cofinal em $\mathcal{A} \subseteq[\omega]^{\omega}$, com $|D|+|\mathcal{A}|=\mathfrak{d}_{2}$. Para cada $g \in D$ e $A \in \mathcal{A}$, definimos $g_{A} \in{ }^{\omega} \omega$ pondo, para cada $k<\omega, g_{A}(k)=g(\min \{a \in A: k \leqslant$ a\}). Considere agora $D^{\prime}=\left\{g_{A}: g \in D, A \in \mathcal{A}\right\}$. Então $\left|D^{\prime}\right| \leqslant \mathfrak{d}_{2}$ e é suficiente mostrar que $D^{\prime}$ é cofinal em $\left\langle{ }^{\omega} \omega, \leqslant\right\rangle$ para obtermos o desejado.

Pois bem; seja $f \in{ }^{\omega} \omega$ qualquer, e seja $h$ uma função não-decrescente que satisfaça $f \leqslant h$. Existem então $g \in D$ e $A \in \mathcal{A}$ tais que $h \leqslant g$ em $A$. Afirmamos que $f \leqslant g_{A}$. De fato: se $k<\omega$ é arbitrário e $m=\min \{a \in A: k \leqslant a\}$, então $f(k) \leqslant h(k) \leqslant h(m) \leqslant$ $g(m)=g_{A}(k)$.

O próximo teorema dá duas caracterizações alternativas para $t$.

Teorema $2.4 \quad t=\mathfrak{t}_{1}=\mathfrak{t}_{2}$, onde

$$
\begin{gathered}
\mathfrak{t}_{1}=\min \left\{\kappa: \text { Existe uma aplicação } T: \kappa \mapsto[\omega]^{\omega} \text { satisfazendo }(\forall \xi, \eta<\kappa)[\xi<\eta \Rightarrow\right. \\
\left.\left.T_{\eta} \subseteq^{*} T_{\xi}\right] \text { e im }(T) \text { não possui pseudo-intersecção infinita }\right\} \\
\mathfrak{t}_{2}=\min \left\{|\mathcal{T}|: \mathcal{T} \subseteq[\omega]^{\omega} \text { é bem-ordenado por } \subset^{*},(\forall T \in \mathcal{T})[|\omega \backslash T|=\omega] e\right. \\
\left.\left(\forall X \in[\omega]^{\omega}\right)(\exists T \in \mathcal{T})[|X \cap T|=\omega]\right\}
\end{gathered}
$$

O leitor mais atento já deve ter percebido que a única diferença entre as definições de $\mathfrak{t}$ e $\mathfrak{t}_{1}$ é que usamos " $\subseteq$ " em $\mathfrak{t}_{1}$, em vez de " $C^{*}$ ".

Demonstração Uma torre de tamanho $t$ está nas condições da definição de $t_{1}$, donde $\mathfrak{t}_{1} \leqslant \mathfrak{t}$. A igualdade $\mathfrak{t}=\mathfrak{t}_{2}$ é consequência imediata de passagens ao complementar: uma família $\mathcal{T}$ nas condições da definição de $\mathfrak{t}_{2}$, com tipo de ordem de $\left\langle\mathcal{T}, \subset^{*}\right\rangle$ igual a $\zeta$, é tal que $\{\omega \backslash T: T \in \mathcal{T}\}$ é claramente uma torre de tipo de ordem também igual a $\zeta$, donde $\mathfrak{t} \leqslant \mathfrak{t}_{2} ;$ já para $\mathfrak{t}_{2} \leqslant \mathfrak{t}$ observe que se $\mathcal{A}=\left\{A_{\xi}: \xi<\mathfrak{t}\right\}$ é uma torre de tipo de ordem $\mathfrak{t}$, posso supor sem nenhuma perda de generalidade que $A_{0}$ é tal que $\omega \backslash A_{0}$ é infinito (pois, se 
não o for, descarto-o e apenas os índices menores que $\omega$ são trocados na nossa enumeração de $\mathcal{A}$ ) e daí $\left\{\omega \backslash A_{\xi}: \xi<\mathfrak{t}\right\}$ está claramente nas condições de $\mathfrak{t}_{2}$.

Para $t \leqslant \mathfrak{t}_{1}$, a idéia é, a partir de uma família $\left\{T_{\xi}: \xi<\zeta\right\} \subseteq[\omega]^{\omega}$, bem-ordenada por $\supseteq^{*}$, sem pseudo-intersecção infinita e com enumeração dada pelo seu tipo de ordem ( com $\left.|\zeta|=\mathfrak{t}_{1}\right)$, descartar "alguns" elementos e obter uma família em que não apareçam dois elementos de uma mesma classe de equivalência de $=^{*}$. Formalizando a idéia, definimos $I=\left\{\eta<\zeta:(\exists \xi<\eta)\left[T_{\xi}={ }^{*} T_{\eta}\right]\right\}$. Como $\left\{T_{\xi}: \xi<\zeta\right\}$ não tem pseudo-intersecção infinita, $\zeta \backslash I$ é cofinal em $\zeta$ e temos que $i m(T \uparrow(\zeta \backslash I))$ é uma torre, conseqüentemente $\mathfrak{t} \leqslant|\zeta \backslash I| \leqslant|\zeta|=\mathfrak{t}_{1}$.

Agora, pagaremos nossa principal dívida da seção anterior, que é a igualdade $\mathfrak{p}=\mathfrak{p}_{2}$ do teorema abaixo, da qual o Fato 2.2 , usado na demonstração de que $\mathfrak{p}$ é regular, é consequência imediata.

Teorema $2.5 \quad \mathfrak{p}=\mathfrak{p}_{1}=\mathfrak{p}_{2}$, onde

$$
\begin{aligned}
& \mathfrak{p}_{1}=\min \left\{|\mathcal{T}|: \mathcal{T} \subseteq[\omega]^{\omega}, \forall \mathcal{F} \in[\mathcal{T}]^{<\omega}[|\omega \backslash \cup \mathcal{F}|=\omega] \text { e }\left(\forall X \in[\omega]^{\omega}\right)(\exists T \in\right. \\
&\mathcal{T})[|X \cap T|=\omega]\} \\
& \mathfrak{p}_{2}=\min \{|\mathcal{A}|+|\mathcal{B}|: \mathcal{A}, \mathcal{B} \subseteq \mathcal{P}(\omega), \mathcal{B} \neq \emptyset,(\forall B \in \mathcal{B})[\mathcal{A} \uparrow B \text { possui a s.f.i.p. }] \\
& \text { e não existe uma pseudo-interseç̧ão } I \text { de } \mathcal{A} \text { satisfazendo }(\forall B \in \mathcal{B})[\text { I } \cap B \\
&\text { é infinito }]\}
\end{aligned}
$$

Demonstração A igualdade $\mathfrak{p}=\mathfrak{p}_{1}$, analogamente a $\mathfrak{t}=\mathfrak{t}_{2}$ do teorema anterior, segue diretamente de passagens ao complementar $\left(\mathfrak{p} \leqslant \mathfrak{p}_{1}\right.$ é óbvia e, para $\mathfrak{p}_{1} \leqslant \mathfrak{p}$, observamos que, se $\mathcal{A} \subseteq[\omega]^{\omega}$ é tal que $|\mathcal{A}|=\mathfrak{p}, \mathcal{A}$ satisfaz a s.f.i.p. e não possui pseudo-intersecção infinita, então $\{\omega \backslash A: A \in \mathcal{A}$ e $\omega \backslash A$ é infinito $\}$ tem cardinalidade $\mathfrak{p}$ (já que $\{\omega \backslash A: A \in \mathcal{A}$ e $\omega \backslash A$ é finito $\}$ é no máximo enumerável) e satisfaz as condições da definição de $\mathfrak{p}_{1}$ ).

Para $\mathfrak{p}_{2} \leqslant \mathfrak{p}$, tome $\mathcal{A} \operatorname{com}|\mathcal{A}|=\mathfrak{p}, \mathcal{A}$ satisfazendo a s.f.i.p. e sem pseudointersecção infinita e tome $\mathcal{B}=\left\{\cap \mathcal{F}: \mathcal{F} \in[\mathcal{A}]^{<\omega}\right\}$. Então $|\mathcal{B}| \leqslant \mathfrak{p},(\forall B \in \mathcal{B})[\mathcal{A} \mid B=$ $\{A \cap B: A \in \mathcal{A}\}$ possui a s.f.i.p. ] e $\mathcal{A}$ não pode possuir pseudo-intersecção satisfazendo $(\forall B \in \mathcal{B})[I \cap B$ é infinito ] pois nesse caso $I$ seria também infinito.

Prova de $\mathfrak{p} \leqslant \mathfrak{p}_{2}$ : Mostraremos que não pode ocorrer $\mathfrak{p}_{2}<\mathfrak{p}$ pois, dados $\mathcal{A}, \mathcal{B} \subseteq \mathcal{P}(\omega)$ $\operatorname{com}|\mathcal{A}|+|\mathcal{B}|<\mathfrak{p}, \mathcal{B} \neq \emptyset$ e $(\forall B \in \mathcal{B})[\mathcal{A}\lceil B$ possui a s.f.i.p. ], exibiremos uma 
pseudo-intersecção infinita de $\mathcal{A}$ cuja intersecção com qualquer elemento de $\mathcal{B}$ é infinita.

De fato: sejam $\mathcal{A}$ e $\mathcal{B}$ como no parágrafo anterior. Definimos as subfamílias $\mathcal{H}_{1}$ e $\mathcal{H}_{2}$ de $\mathcal{P}\left([\omega]^{<\omega}\right)$ pondo

$$
\begin{aligned}
& \mathcal{H}_{1}=\left\{[A]^{<\omega}: A \in \mathcal{A}\right\} \\
& \mathcal{H}_{2}=\left\{\left\{F \in[\omega \backslash n]^{<\omega}: F \cap B \neq \emptyset\right\}: B \in \mathcal{B}, n<\omega\right\}
\end{aligned}
$$

Afirmamos que $\mathcal{H}_{1} \cup \mathcal{H}_{2}$ possui a s.f.i.p. Note que estamos tratando da s.f.i.p. em $\mathcal{P}\left([\omega]^{<\omega}\right)$, não em $\mathcal{P}(\omega)$. A idéia que surgirá em breve é que, como $\left|[\omega]^{<\omega}\right|=\omega$, podemos identificar $\omega \operatorname{com}[\omega]^{<\omega}$ e, de $\left|\mathcal{H}_{1} \cup \mathcal{H}_{2}\right|<\mathfrak{p}$ (o que é evidente), encontraremos uma pseudointersecção infinita $\mathcal{I}$ de $\mathcal{H}_{1} \cup \mathcal{H}_{2}$ em $\mathcal{P}\left([\omega]^{<\omega}\right)$. Dividiremos a verificação de que $\mathcal{H}_{1} \cup \mathcal{H}_{2}$ possui a s.f.i.p. em três casos.

Caso 1: A subfamília finita e não-vazia de $\mathcal{H}_{1} \cup \mathcal{H}_{2}$ está contida em $\mathcal{H}_{1}$. Nesse caso, note que nossa principal hipótese implica que $\mathcal{A}$ possui a s.f.i.p. Assim, se $A_{1}, \ldots, A_{k} \in \mathcal{A}$, então $\bigcap_{i=1}^{k}\left[A_{i}\right]^{<\omega}$ é infinito pois contém todos os subconjuntos finitos do conjunto infinito $\bigcap_{i=1}^{k} A_{i}$.

Caso 2: A subfamília finita e não-vazia está contida em $\mathcal{H}_{2}$. Nesse caso, note que nossa principal hipótese implica que todos os elementos de $\mathcal{B}$ são infinitos. Assim, se $B_{1}, \ldots, B_{k} \in \mathcal{B}, n_{1}, \ldots, n_{k} \in \omega$, tomo $n^{*}=\max \left\{n_{i}: 1 \leqslant i \leqslant k\right\}$ e tomo $b_{i} \in B_{i}$ com $b_{i}>n^{*}$ para $i=1, \ldots, k$. Segue que qualquer subconjunto finito de $\omega \backslash n^{*}$ que contenha $\left\{b_{i}: 1 \leqslant i \leqslant k\right\}$ pertencerá a $\bigcap_{i=1}^{k}\left\{F \in\left[\omega \backslash n_{i}\right]^{<\omega}: F \cap B_{i} \neq \emptyset\right\}$.

Caso 3: A subfamília finita e não vazia contém elementos tanto de $\mathcal{H}_{1}$ como de $\mathcal{H}_{2}$. Nesse caso, escrevemos $\mathcal{F} \in\left[\mathcal{H}_{1} \cup \mathcal{H}_{2}\right]^{<\omega}$ na forma $\mathcal{F}=\mathcal{F}_{1} \cup \mathcal{F}_{2}, \emptyset \neq \mathcal{F}_{i} \in\left[\mathcal{H}_{i}\right]^{<\omega}$ para $i=1,2$. Se

$$
\begin{aligned}
& \mathcal{F}_{1}=\left\{\left[A_{i}\right]^{<\omega}: A_{i} \in \mathcal{A} \text { para } 1 \leqslant i \leqslant k\right\} \\
& \mathcal{F}_{2}=\left\{\left\{F \in\left[\omega \backslash n_{j}\right]^{<\omega}: F \cap B_{j} \neq \emptyset\right\}: n_{j}<\omega, B_{j} \in \mathcal{B}, 1 \leqslant j \leqslant m\right\}
\end{aligned}
$$

então nossa principal hipótese implica que $C_{j}=\left(\bigcap_{i=1}^{k} A_{i}\right) \cap B_{j}$ é infinito, para $1 \leqslant j \leqslant m$. Seja então $n^{*}=\max \left\{n_{j}: 1 \leqslant j \leqslant m\right\}$. Escolhendo $b_{j} \in C_{j} \operatorname{com} b_{j}>n^{*}$ para $1 \leqslant j \leqslant m$ 
segue imediatamente que qualquer subconjunto finito de $\left(\bigcap_{i=1}^{k} A_{i}\right) \backslash n^{*}$ que contenha $\left\{b_{j}\right.$ : $1 \leqslant j \leqslant m\}$ pertence a $\bigcap \mathcal{F}$.

Assim, $\mathcal{H}_{1} \cup \mathcal{H}_{2}$ é uma subfamília de $\mathcal{P}\left([\omega]^{<\omega}\right)$ que possui a s.f.i.p. e $\left|\mathcal{H}_{1} \cup \mathcal{H}_{2}\right|<$ p. Usando a identificação entre $\omega$ e $[\omega]^{<\omega}$ à qual nos referimos anteriormente, existe $\mathcal{I} \in \mathcal{P}\left([\omega]^{<\omega}\right)$ que é uma pseudo-intersecção infinita de $\mathcal{H}_{1} \cup \mathcal{H}_{2}$. Afirmamos que $\bigcup \mathcal{I}$ é uma pseudo-intersecção infinita de $\mathcal{A}$ cuja intersecção com qualquer elemento de $\mathcal{B}$ é infinita.

De fato: dado $A \in \mathcal{A}$ qualquer, temos que $\mathcal{I} \backslash[A]^{<\omega}$ é finito, donde $(\bigcup \mathcal{I}) \backslash A \subseteq$ $\bigcup\left(\mathcal{I} \backslash[A]^{<\omega}\right)$ que é finito, logo $(\bigcup \mathcal{I}) \subseteq^{*} A$ e $\bigcup \mathcal{I}$ é pseudo-intersecção infinita de $\mathcal{A}$. Tomemos agora $B \in \mathcal{B}$ arbitrário e $n<\omega$ qualquer; então $\mathcal{I} \backslash\left\{F \in[\omega \backslash n]^{<\omega}: F \cap B \neq \emptyset\right\}$ é finito. Logo, para todos exceto no máximo finitos $I \in \mathcal{I}$, temos $\min (I) \geqslant n$ e $I \cap B \neq \emptyset$; como $n<\omega$ é arbitrário, $(\bigcup \mathcal{I}) \cap B$ é infinito. Como $B$ também é arbitrário, mostramos o desejado.

\subsection{Resultados de consistência e de independência}

Nesta seção, faremos alguns comentários a respeito de resultados (e problemas abertos) relativos a problemas de consistência e de independência que envolvem nossos cardinais. Em geral, não apresentaremos demonstrações desses resultados, e por isso respeitaremos a numeração com a qual eles foram publicados em suas respectivas obras de referência.

$\mathrm{O}$ primeiro resultado de consistência a respeito dos pequenos cardinais $\mathfrak{a}, \mathfrak{b}, \mathfrak{d}$, $\mathfrak{p}, \mathfrak{s}$ e $\mathfrak{t}$ é óbvio: em modelos nos quais vale $C H, \omega_{1}$ e $\mathfrak{c}$ coincidem e portanto todos os nossos cardinais são "espremidos pelo colapsamento", isto é, sob $C H$ vale $\omega_{1}=\kappa=\mathfrak{c}$ para $\kappa \in\{\mathfrak{a}, \mathfrak{b}, \mathfrak{o}, \mathfrak{p}, \mathfrak{s}, \mathfrak{t}\}$. Em particular, já é consistente que $\mathfrak{a}, \mathfrak{d}$ e $\mathfrak{s}$ sejam cardinais regulares. Porém, veremos que muitas outras situações envolvendo os seis cardinais podem ocorrer (no sentido de que "é consistente que ocorram").

Um resultado muito importante no contexto do que tratamos nesta seção envolve o chamado Axioma de Martin. O Axioma de Martin, freqüentemente denotado por " $M A$ ", é um axioma de enunciado combinatório e não muito intuitivo e que funciona co- 
mo uma espécie de "forcing dentro do modelo", logo a linguagem de "p.o.'s", "cadeias e anticadeias", "filtros e densos" torna-se necessária.

Uma p.o. (partial order) é um par $\langle\mathbb{P}, \leqslant\rangle$ tal que $\mathbb{P} \neq \emptyset$ e $\leqslant$ é uma relação em $\mathbb{P}$ transitiva e reflexiva. Na verdade, esta definição praticamente coincide com a que demos para "pré-ordens", mas nos contextos em que o forcing e/ou $M A$ são utilizados as ordens parciais $\mathbb{P}$ são em geral definidas sem a exigência de antissimetria.

Um subconjunto $D \subseteq \mathbb{P}$ é dito denso se $(\forall p \in \mathbb{P})(\exists d \in D)[d \leqslant p]$. Um subconjunto $G \subseteq \mathbb{P}$ é dito um filtro se: i) $(\forall g \in G)(\forall p \in \mathbb{P})[g \leqslant p \Rightarrow p \in G]$; e ii) $\left(\forall g, g^{\prime} \in G\right)(\exists r \in G)\left[(r \leqslant g) \wedge\left(r \leqslant g^{\prime}\right)\right]$. Dois elementos $p, q \in \mathbb{P}$ são ditos compatíveis se $(\exists r \in \mathbb{P})[(r \leqslant p) \wedge(r \leqslant q)]$; caso contrário, eles são ditos incompatíveis, o que se denota por $p \perp q$. Uma cadeia em $P$ é um subconjunto de $\mathbb{P}$ totalmente ordenado por $\leqslant \mathrm{e}$ uma anticadeia em $\mathbb{P}$ é um subconjunto de $\mathbb{P}$ no qual quaisquer dois elementos distintos são incompatíveis. Dizemos que $\mathbb{P}$ satisfaz a propriedade c.c.c. (ou, simplesmente, $\mathbb{P}$ é c.c.c.) se qualquer anticadeia em $\mathbb{P}$ é no máximo enumerável. "c.c.c." denota a chamada countable chain condition, a condição de cadeia enumerável, apesar de ser uma condição que se aplica a anticadeias; há quem defenda que a terminologia correta seria "c.a.c.". No entanto, a expressão "c.c.c." já está estabelecida e consagrada. Bem, com os termos definidos nos dois últimos parágrafos já podemos enunciar o Axioma de Martin.

Seja $M A(\kappa)$ a asserção: "Se $\mathbb{P}$ é uma p.o. c.c.c. e $\mathcal{D}$ é uma família de densos de $\mathbb{P}$ tal que $|\mathcal{D}| \leqslant \kappa$, então existe um filtro $G$ tal que $G \cap D \neq \emptyset$ para todo $D \in \mathcal{D}$ ". Sabe-se que $M A(\omega)$ é verdadeiro e que $M A(\mathfrak{c})$ é falso ([Kun\$0], Lema 2.6). Seja então

$$
\mathfrak{m}=\min \{\kappa: M A(\kappa) \text { é falso }\}
$$

O Axioma de Martin (MA) é a asserção " $m=\mathfrak{c}$ ", ou, equivalentemente, $M A$ é a sentença $\forall \lambda[\lambda$ é cardinal e $\omega \leqslant \lambda<\mathfrak{c} \Rightarrow M A(\lambda)]$.

$C H$ implica $M A$, obviamente. No entanto, $M A+\neg C H$ é consistente ([Kun80], VIII §6).

Veremos agora que, sob $M A, \mathfrak{p}=\mathfrak{c}$ (e, em virtude das desigualdades provadas no Teo. $2.1, \kappa=\mathfrak{c}$ para $\kappa \in\{\mathfrak{a}, \mathfrak{b}, \mathfrak{d}, \mathfrak{p}, \mathfrak{s}, \mathfrak{t}\}$.). Inicialmente, enunciemos aqui o Teorema 2.15 do livro de Kunen ([Kun80]), que é devido a Solovay:

Teorema 2.15 ([Kun80], "Lema de Solovay") Assuma $M A(\kappa) . \operatorname{Sejam~} \mathcal{A}, \mathcal{C} \subseteq \mathcal{P}(\omega)$, 
com $|\mathcal{A}| \leqslant \kappa,|\mathcal{C}| \leqslant \kappa$, e assuma que, para todo $y \in \mathcal{C}$ e para todo $F \subset \mathcal{A}$ finito, $|y \backslash \bigcup F|=\omega$; então existe $d \subset \omega$ tal que $(\forall x \in \mathcal{A})[d \cap x \mid<\omega]$ e $(\forall y \in \mathcal{C})[|d \cap y|=\omega]$.

A demonstração do Teorema acima consta na pag.57 de [Kun80]. Mas, comparando o enunciado desse teorema com a definição dada ao cardinal $\mathfrak{p}_{2}$ na seção anterior (Teorema 2.5), é imediato que, assumindo $M A, \mathfrak{p}=\mathfrak{p}_{2}=\mathfrak{c}$ (na definição de $\mathfrak{p}_{2}$, trocando $\mathcal{A}$ por $\mathcal{A}^{\prime}=\{\omega \backslash A: A \in \mathcal{A}\}$, tomando $\mathcal{B}=\mathcal{C}$ e aplicando o Teorema que acabamos de enunciar, conclui-se que $M A\left(\mathfrak{p}_{2}\right)$ é falso donde $\left.\mathfrak{c} \leqslant \mathfrak{p}_{2}=\mathfrak{p} \leqslant \mathfrak{c}\right)$. Na verdade, a hipótese " $M A$ " pode ser enfraquecida para " $M A_{\sigma-c e n t r a d a s " . ~} M A_{\sigma-c e n t r a d a s}$ é uma forma (estritamente mais fraca) do Axioma de Martin que restringe às chamadas p.o.'s $\sigma$-centradas as asserções do tipo "MA( $\lambda)$ vale" (uma p.o. IP é dita $\sigma$-centrada se puder ser escrita como uma união enumerável de subconjuntos centrados, sendo que um subconjunto $B \subseteq \mathbb{P}$ é dito centrado se todo $F \subset B$ finito possui limitantes inferiores em $\mathbb{P}$.). É claro que p.o.'s $\sigma$-centradas são c.c.c., donde $M A \Rightarrow M A_{\sigma \text {-centradas }}$, mas não vale a recíproca (ver comentários e referências na pág.124 de [vD84]). No quanto à relação existente entre $M A_{\sigma \text {-centradas }}$ e o cardinal $\mathfrak{p}$, podemos afirmar mais do que " $M A_{\sigma-\text { centradas }} \Rightarrow \mathfrak{p}=\mathfrak{c}$ "; Bell mostrou em 1981 que "MA $A_{\sigma-\text { centradas }}(\kappa)$ " e " $\kappa<\mathfrak{p}$ " são equivalentes (ver [Fre84], $14 \mathrm{C})$.

Observamos aqui que, em vista de $M A \Rightarrow \mathfrak{p}=\mathfrak{c}$ e do item $a$ ) do Teo.2.1, , em modelos de $M A+\neg C H$ tem-se $\omega_{1}<\kappa=\mathfrak{c}$ para $\kappa \in\{\mathfrak{a}, \mathfrak{b}, \mathfrak{d}, \mathfrak{p}, \mathfrak{s}, \mathfrak{t}\}$.

O seguinte teorema, o primeiro da seção relativa a resultados de consistência do artigo de van Douwen ([vD84]), encerra a questão no que se refere a consistência das asserções " $\omega_{1}=\kappa=\mathfrak{c}$ ", " $\omega_{1}=\kappa<\mathfrak{c}$ ", " $\omega_{1}<\kappa<\mathfrak{c}$ ", " $\omega_{1}<\kappa=\mathfrak{c}$ " para $\kappa \in\{\mathfrak{a}, \mathfrak{b}, \mathfrak{d}, \mathfrak{p}, \mathfrak{s}, \mathfrak{t}\}$.

Teorema 5.1 ([vD84]) Sejam $\kappa$ e $\lambda$ cardinais regulares com $\omega_{1} \leqslant \kappa \leqslant \lambda$. É consistente com $Z F C$ que $\mathfrak{c}=\lambda$ e $\mathfrak{a}=\mathfrak{b}=\mathfrak{d}=\mathfrak{p}=\mathfrak{s}=\mathfrak{t}=\kappa$.

Para o caso em que $\kappa=\lambda$, a demonstração utiliza que " $M A+\mathfrak{c}=\lambda$ " é consistente (ver [Kun80], VIII $\S 6$ ) e " $M A \Rightarrow \mathfrak{p}=\mathfrak{c}$ ". Para $\kappa<\lambda$, o resultado segue a partir de uma aplicação de forcing iterado.

Até o momento, as discussões sobre consistência têm nos levado a situações nas 
quais os seis cardinais coincidem. Veremos agora que é consistente que eles não sejam todos iguais.

Tendo-se seis cardinais, existem 30 perguntas do tipo "é consistente $\kappa<\lambda$ ?". Com o Teorema 2.1, o número de perguntas que continuam fazendo sentido cai para 18 (a saber: é consistente que $\mathfrak{a}<\mathfrak{d} ? \mathfrak{a}<\mathfrak{s} ? \mathfrak{b}<\mathfrak{a} ? \mathfrak{b}<\mathfrak{d} ? \mathfrak{b}<\mathfrak{s}$ ? $\mathfrak{d}<\mathfrak{a}$ ? $\mathfrak{p}<\mathfrak{a}$ ? $\mathfrak{p}<\mathfrak{b} ? \mathfrak{p}<\mathfrak{d} ? \mathfrak{p}<\mathfrak{s} ? \mathfrak{p}<\mathfrak{t} ? \mathfrak{s}<\mathfrak{a} ? \mathfrak{s}<\mathfrak{b} ? \mathfrak{s}<\mathfrak{d} ? \mathfrak{t}<\mathfrak{a} ? \mathfrak{t}<\mathfrak{b} ? \mathfrak{t}<\mathfrak{d} ? \mathfrak{t}<\mathfrak{s} ?)$

Vejamos como essas perguntas foram quase todas resolvidas. Todas as soluções foram obtidas por forcing. Os próximos três teoremas constam em [vD84].

Teorema 5.2 ([vD84]) É consistente com ZFC que $\mathfrak{a}<\mathfrak{d}$ (e portanto $\mathfrak{b}<\mathfrak{d}$, já que $\mathfrak{b} \leqslant \mathfrak{a})$.

Para esta prova de consistência, construiu-se um modelo no qual $\omega_{1}=\mathfrak{b}=\mathfrak{a}<$ $\mathfrak{d}=\mathfrak{c}$. Duas das 18 perguntas já foram respondidas ( $\mathfrak{a}<\mathfrak{d}$ e $\mathfrak{b}<\mathfrak{d}$ são consistentes.).

Teorema 5.3 ([vD84]) É consistente com $Z F C$ que $\mathfrak{t}<\mathfrak{b}$.

Para esta prova de consistência, construiu-se um modelo no qual $\omega_{1}=\mathfrak{t}<\mathfrak{b}=$ $\mathfrak{d}=\omega_{2}=\mathfrak{c}$. Caem agora mais seis das 18 perguntas: $\mathfrak{t}<\mathfrak{b}, \mathfrak{t}<\mathfrak{a}, \mathfrak{t}<\mathfrak{d}, \mathfrak{p}<\mathfrak{b}, \mathfrak{p}<\mathfrak{a}$ e $\mathfrak{p}<\mathfrak{d}$ são consistentes.

Teorema 5.4 ([vD84]) É consistente com $Z F C$ que $\mathfrak{t}<\mathfrak{s}$.

Para esta prova de consistência, construiu-se um modelo no qual $\omega_{1}=\mathfrak{t}<\mathfrak{s}=$ $\omega_{2}=\mathfrak{c}$. Mais duas perguntas caíram: $\mathfrak{t}<\mathfrak{s}$ e $\mathfrak{p}<\mathfrak{s}$ são consistentes.

As demonstrações dos Teoremas 5.2 e 5.4 possuem ainda os seguintes corolários, respectivamente: é consistente que $\mathfrak{d}$ seja singular (logo, a regularidade do cardinal $\mathfrak{d}$ é independente de $Z F C$ ) e é consistente que $2^{\mathrm{t}}<2^{\mathrm{s}}$. Estes três teoremas $-5.2,5.3$ e 5.4 - e mais os dois corolários encerram o que se sabia no quanto às "comparações entre os cardinais" até a publicação do artigo de van Douwen; ainda estavam em aberto $\delta$ das 18 perguntas do tipo "é consistente $\kappa<\lambda$ ?" e não se sabia se existiam modelos nos quais a e $\mathfrak{s}$ fossem cardinais regulares.

Esta última questão continua em aberto e é o Problema 334 do Open Problems ([Vau90], p.205.). Boa parte das outras questões " $\kappa<\lambda$ " pendentes foi resolvida por 
Shelah ([She84]), praticamente no mesmo momento em que o artigo de van Douwen era publicado.

Teorema 1.15 ([She84]) É consistente com ZFC que $\omega_{1}=\mathfrak{b}<\mathfrak{a}=\mathfrak{s}=\mathfrak{c}=\omega_{2}$.

Caem com o Teorema 1.15 duas perguntas $(\mathfrak{b}<\mathfrak{a}, \mathfrak{b}<\mathfrak{s}$ são consistentes.)

Teorema 3.1 ([She84]) É consistente com ZFC que $\omega_{1}=\mathfrak{b}=\mathfrak{a}<\mathfrak{s}=\mathfrak{c}=\omega_{2}$.

Com o Teorema 3.1, cai apenas uma das perguntas ( $\mathfrak{a}<\mathfrak{s}$ é consistente.).

Conclusão 4.4 ([She84]) É consistente com ZFC que $\omega_{1}=\mathfrak{s}<\mathfrak{d}=\mathfrak{b}=\omega_{2}=2^{\omega_{1}}=\mathfrak{c}$.

E agora caem três perguntas: $\mathfrak{s}<\mathfrak{b}, \mathfrak{s}<\mathfrak{d}$ e $\mathfrak{s}<\mathfrak{a}$ são consistentes.

O leitor mais curioso já deve ter contado quantas das 18 perguntas continuam sem solução: sim, são apenas duas. a saber: $\mathfrak{d}<\mathfrak{a}$ é consistente ? $\mathfrak{p}<\mathfrak{t}$ é consistente ? Essas questões continuam em aberto até o momento e fazem parte do Problema 333 do Open Problems ([Vau90], pag.198).

Logo, os problemas em aberto a respeito de questões de consistência que envolvem nossos seis cardinais são: podem a e $\mathfrak{s}$ serem singulares ? É consistente $\mathfrak{d}<\mathfrak{a}$ ? É consistente $\mathfrak{p}<t$ ? Observamos que, a partir dos diversos problemas que já estão resolvidos, podemos enunciar o seguinte resultado, no mínimo interessante: as relações de ordem existentes entre $\mathfrak{a}$ e $\mathfrak{s}$ e entre $\mathfrak{b}$ e $\mathfrak{s}$ são independentes de $Z F C$, já que as asserções $\mathfrak{a}<\mathfrak{s}$, $\mathfrak{s}<\mathfrak{a}, \mathfrak{b}<\mathfrak{s}$ e $\mathfrak{s}<\mathfrak{b}$ são todas consistentes.

\section{Notas}

Boa parte dos resultados expostos nas três primeiras seções deste capítulo constam na seção 3 de [vD84]; tivemos porém que apresentar a redação de alguns "fatos não-explicitados" por van Douwen ou de algumas demonstrações que constavam como exercício. Por exemplo, quase todas as verificações de " $\mu$ está bem definido e $\omega_{1} \leqslant \mu \leqslant \mathfrak{c}$ para $\mu$ entre nossos seis cardinais" foram redigidas por nós ( a menos de " $\omega_{1} \leqslant \mathfrak{p}$ ", que 
consta no Teo.3.1 de [vD84], e de " $\omega_{1} \leqslant \mathfrak{s}$ " que está praticamente como no original de Booth ([Boo76]) ). Modificamos também a ordem de apresentação dos resultados, pois nos pareceu mais natural e instrutivo apresentar os resultados básicos de $Z F C$ (Teo.2.1) antes das definições alternativas para os cardinais, diferentemente do que fez van Douwen em [vD84]; dessa forma, alguns itens do Teo.2.1 foram demonstrados de modo um pouco diferente. "b $\leqslant \mathfrak{a}$ " está como no original de Solomon ([Sol77]) e " $\mathfrak{p}=\omega_{1} \Leftrightarrow \mathfrak{t}=\omega_{1}$ " está praticamente como em [Rot48], conforme destacou Fremlin em [Fre84]. A demonstração do item $d$ ) do Teo.2.1 consta como exercício em [vD84], e efetivamente fizemos esse exercício. O Fato 2.1 teve enunciado levemente modificado (não é verdade, sob nossa definição de $\leqslant^{*}$, que " $b_{A}<^{*} f \Rightarrow(\exists m<\omega)(\forall n<\omega)\left[n \geqslant m \Rightarrow f(n)>b_{A}(n)\right]$ ", mas isso é facilmente resolvido tomando-se $g=f+1$ e aplicando novamente que $\mathcal{F}$ é dominante.). As Observações 2.1 e 2.2 não são explicitadas em [vD84]. A demonstração que fizemos de $\mathfrak{b} \leqslant \mathfrak{b}_{7}$ no Teo.2.2 formaliza alguns "without loss of generality" de van Douwen.

A adaptação da prova de W.Rudin em [Rud56] (trocando " $C H$ " por " $\mathfrak{p}=\mathfrak{c}$ " como hipótese que implica na existência de $P$-pontos) é simples. Para redigir os comentários sobre independência e consistência, o artigo de Vaughan no Open Problems ([Vau90]) forneceu quase todas as informações necessárias. Mais comentários históricos e bibliográficos a respeito dos nossos seis cardinais podem ser vistos na pág.123 de [vD84]. 


\section{Capítulo 3}

\section{Gaps em $\left({ }^{\omega} \omega,<^{*}\right)$ e $\left(\mathcal{P}(\omega), \subset^{*}\right)$}

Neste capítulo, introduziremos os conceitos e resultados básicos sobre gaps que serão utilizados em aplicações à topologia (no último capítulo deste trabalho) e no próprio estudo de alguns dos nossos pequenos cardinais.

Este capítulo se pretende breve e apenas apresenta o instrumental básico para o que será feito posteriormente (sendo que determinados resultados clássicos são respeitosamente revisitados). Ao leitor interessado num estudo mais profundo sobre gaps, recomendamos o artigo de Scheepers ([Sch93]).

\subsection{Gaps em conjuntos parcialmente ordenados}

Estruturas semelhantes a "lacunas" são comuns mesmo em matemática elementar. Qualquer bom aluno de graduação em matemática sabe que existem, por exemplo, sequências de racionais $\left\langle x_{n}\right\rangle_{n<\omega}$ e $\left\langle y_{n}\right\rangle_{n<\omega}$, respectivamente estritamente crescente e estritamente decrescente, convergindo para $\sqrt{2}$ (ou para qualquer número irracional previamente dado). Considerando-se $Q$ com sua ordem usual, temos $X=\left\{x_{n}: n<\omega\right\}$ bem-ordenado por $<$ e $Y=\left\{y_{n}: n<\omega\right\}$ bem-ordenado por $>$, ambos com tipo de ordem $\omega$; podemos cometer um abuso de linguagem aqui e dizer que $Y$ está ordenado por < com "tipo de ordem" $\omega^{*}$ (onde o asterisco indica a inversão de ordem no ordinal $\omega$ e a expressão 'tipo de ordem' aparece entre aspas para lembrarmos que $<$ não é boa-ordem 
em $Y$ ). Note que, se $m<n<\omega$, então $x_{m}<x_{n}<y_{n}<y_{m}$ e não existe nenhum número racional $z$ que satisfaça $x_{i}<z<y_{j}$ para todos os $i, j<\omega$. Temos uma "lacuna" aqui. Basicamente, a construção dos números reais consiste em destruir esse tipo de "lacuna" que a estrutura $\langle Q, \leqslant\rangle$ possui. Neste nosso exemplo elementar, a ordem $<$ é uma ordem total. No entanto, definiremos o conceito de gap ("lacuna", em inglês) para uma ordem parcial qualquer.

Definição 3.1 (i) Seja $\langle P, \leqslant\rangle$ uma ordem parcial qualquer. Dados dois subconjuntos de $P, X=\left\{x_{\zeta}: \zeta<\alpha\right\}$ e $Y=\left\{y_{\xi}: \xi<\beta\right\}$, dizemos que $\langle X, Y\rangle$ é um $\left\langle\alpha, \beta^{*}\right\rangle$-pré-gap em $\langle P, \leqslant\rangle$ se

$$
x_{\zeta_{1}}<x_{\zeta_{2}}<y_{\xi_{2}}<y_{\xi_{1}}
$$

para quaisquer $\zeta_{1}<\zeta_{2}<\alpha, \xi_{1}<\xi_{2}<\beta$. Em particular, tem-se que o tipo de ordem de $\langle X,<\rangle$ é a e o "tipo de ordem" de $\langle Y,<\rangle$ é $\beta^{*}$ (isto é, o tipo de ordem de $\langle Y,>\rangle$ é $\beta$ ). .

(ii) $U m\left\langle\alpha, \beta^{*}\right\rangle$-pré-gap como em i) é dito um $\left\langle\alpha, \beta^{*}\right\rangle$-gap em $\langle P, \leqslant\rangle$ se não existir nenhum $z \in P$ que satisfaça $x_{\zeta}<z<y_{\xi}$ para quaisquer $\zeta<\alpha$ e $\xi<\beta$.

Se os ordinais $\alpha$ e $\beta$ não forem relevantes no contexto em questão, diremos simplesmente que $\langle X, Y\rangle$ é um gap.

(iii) Um elemento de $P$ que "testemunhe" o fato de um determinado $\left\langle\alpha, \beta^{*}\right\rangle$-prégap não ser um gap é dito um interpolador do pré-gap.

Gostaríamos de observar que a idéia do "asterisco indicando inversão de ordem em um ordinal" não é uma idéia nova, como pode ter parecido em nosso ingênuo exemplo trivial. Sierpinzky já usava essa notação.

A seguinte proposição nos permitirá sempre supor, no caso em que os conjuntos que determinam os gaps tem tipos de ordem dados por ordinais limite, que esses tipos de ordem são cardinais regulares.

Proposição 3.1 Sejam $\alpha$ e $\beta$ ordinais limite e $\left\langle\left\{x_{\zeta}: \zeta<\alpha\right\},\left\{y_{\xi}: \xi<\beta\right\}\right\rangle$ um $\left\langle\alpha, \beta^{*}\right\rangle$ pré-gap numa ordem parcial $\langle P, \leqslant\rangle$. Se $A$ e $B$ são subconjuntos cofinais de $\alpha$ e $\beta$ respectivamente, então são equivalentes as afirmações:

(i) $\left\langle\left\{x_{\zeta}: \zeta<\alpha\right\},\left\{y_{\xi}: \xi<\beta\right\}\right\rangle$ é um gap.

(ii) $\left\langle\left\{x_{\zeta}: \zeta \in A\right\},\left\{y_{\xi}: \xi \in B\right\}\right\rangle$ é um gap. 
Demonstração $($ ii $) \Rightarrow($ i $)$ : óbvio, pois um interpolador de $\left\langle\left\{x_{\zeta}: \zeta<\alpha\right\},\left\{y_{\xi}: \xi<\beta\right\}\right\rangle$ é também um interpolador de $\left\langle\left\{x_{\zeta}: \zeta \in C\right\},\left\{y_{\xi}: \xi \in D\right\}\right\rangle$ para quaisquer $C \subseteq \alpha$ e $D \subseteq \beta$.

$(i) \Rightarrow(i i)$ : Assumindo $(i)$, mostraremos que a existência de um interpolador para $\left\langle\left\{x_{\zeta}\right.\right.$ : $\left.\zeta \in A\},\left\{y_{\xi}: \xi \in B\right\}\right\rangle$ leva a uma contradição. De fato: suponha que $z$ é um interpolador de $\left\langle\left\{x_{\zeta}: \zeta \in A\right\},\left\{y_{\xi}: \xi \in B\right\}\right\rangle$, e sejam $\eta$ e $\delta$ ordinais satisfazendo $\eta<\alpha, \delta<\beta$. Como $A$ e $B$ são cofinais em $\alpha$ e $\beta$ respectivamente, existem $\zeta \in A$ e $\xi \in B$ tais que $\eta<\zeta$ e $\delta<\xi$. Disso e da hipótese sobre $z$ segue que $x_{\eta}<x_{\zeta}<z<y_{\xi}<y_{\delta}$, e de $\eta$ e $\delta$ arbitrários teríamos que $z$ interpola $\left\langle\left\{x_{\zeta}: \zeta<\alpha\right\},\left\{y_{\xi}: \xi<\beta\right\}\right\rangle$, contradição.

O comentário antes da proposição fica então justificado, se novamente nos lembrarmos que as cofinalidades de ordinais limite são sempre cardinais regulares.

\subsection{Gaps em $\left({ }^{\omega} \omega,<^{*}\right)$ e $\left(\mathcal{P}(\omega), \subset^{*}\right)$}

Como é de se esperar, nosso principal interesse recairá em gaps nas estruturas $\left\langle\mathcal{P}(\omega), \subset^{*}\right\rangle$ e $\left\langle{ }^{\omega} \omega,<^{*}\right\rangle$. O leitor poderá estranhar a frase anterior, pois, de fato, $\subseteq^{*} \mathrm{e} \leqslant *$ não são ordens parciais, mas sim pré-ordens. No entanto, sempre poderemos imaginar que estamos tratando com classes de representantes da relação de equivalência $={ }^{*}$; nessas condições, tais pré-ordens comportam-se como ordens parciais de fato. Para eliminar esse mal-estar, sempre escreveremos $\left\langle\mathcal{P}(\omega), \subset^{*}\right\rangle$ e $\left\langle{ }^{\omega} \omega,<^{*}\right\rangle$ ao invés de $\left\langle\mathcal{P}(\omega), \subseteq^{*}\right\rangle$ e $\left\langle{ }^{\omega} \omega, \leqslant^{*}\right\rangle$, enquanto estivermos tratando de gaps nessas estruturas.

Os gaps em $\left\langle\mathcal{P}(\omega), \subset^{*}\right\rangle$ e $\left\langle{ }^{\omega} \omega,<^{*}\right\rangle$ foram estudados, entre as décadas de 30 e 50, principalmente por Hausdorff e Rothberger. O artigo de Hausdorff de 1936, "Summen von $\aleph_{1}$ Mengen" ([Hau36]), é até hoje "widely quoted" em qualquer tratamento que se dê ao assunto de gaps. Inúmeros artigos de Rothberger nos anos 40 e 50 (entre eles os dois que constam em nossa bibliografia, [Rot41] e [Rot48] ) tratam do assunto, e chamamos a atenção para o fato de que, mesmo com notações e terminologias bastante diferentes das atuais, estão nesses artigos boa parte do que se sabe hoje (em $Z F C$ ) sobre os cardinais $\mathfrak{b}, \mathfrak{p}$ e $\mathfrak{t}$. Mas, quais são as relações entre os gaps e esses pequenos cardinais ? Ainda precisamos trabalhar um pouco mais para explicitá-las. 
O seguinte teorema é uma adaptação de um argumento original de Hausdorff, de 1936.

\section{Teorema 3.1 [ Hausdorff]}

Se $\langle\mathcal{A}, \mathcal{B}\rangle$ é um pré-gap em $\left\langle{ }^{\omega} \omega,<^{*}\right\rangle$ e $\mathcal{A}, \mathcal{B} \subseteq{ }^{\omega} \omega$ são no máximo enumeráveis, então $\langle\mathcal{A}, \mathcal{B}\rangle$ não é um gap, isto é, existe $h \in{ }^{\omega} \omega$ que interpola o pré-gap $\langle\mathcal{A}, \mathcal{B}\rangle$.

Em particular, não existem $\left\langle\omega, \omega^{*}\right\rangle$-gaps em $\left\langle{ }^{\omega} \omega,<^{*}\right\rangle$.

Demonstração Para o caso de $\mathcal{A}$ e $\mathcal{B}$ finitos, basta saber construir interpoladores para $\langle 1,1\rangle$-gaps. Sejam então $f, g \in{ }^{\omega} \omega$ tais que $f<^{*} g$; temos $\{n<\omega: g(n)<f(n)\}$ finito e $\{n<\omega: f(n)<g(n)\}$ infinito. Decompomos então $\omega$ na forma $\omega=\bigcup_{k<\omega} N_{k}$, onde os $N_{k}$ 's são segmentos finitos, disjuntos, contíguos e não-vazios de $\omega$ e tais que, para cada $i<\omega, \bigcup_{k \leqslant i} N_{k}$ é um segmento inicial de $\omega$; a construção deve ser feita de modo que $\{n<\omega: g(n)<f(n)\} \subseteq N_{0}$ e tal que sempre exista um $n_{i} \in N_{i}$ satisfazendo $f\left(n_{i}\right)<g\left(n_{i}\right)$ para cada $i \geqslant 1$ (o que se consegue facilmente, por recursão finita). Definimos então $h \in$ $\omega_{\omega} \omega$ pondo

$$
h(n)= \begin{cases}0 & \text { se } n \in N_{0} \\ f(n) & \text { se } n \in \bigcup_{i<\omega} N_{2 i+1} \\ g(n) & \text { se } n \in \bigcup_{i<\omega} N_{2(i+1)}\end{cases}
$$

Em $\omega \backslash N_{0}, f(n) \leqslant h(n) \leqslant g(n)$ para todo $n$, donde $f \leqslant{ }^{*} h \leqslant{ }^{*} g$, mas, para infinitos $n$ em $\bigcup_{i<\omega} N_{2 i+1}$ tem-se $h(n)<g(n)$, donde $g \mathbb{*}^{*} h$, e para infinitos $n$ em $\bigcup_{i<\omega} N_{2(i+1)}$ tem-se $f(n)<h(n)$, donde $h \mathbb{k}^{*} f$. Portanto $f<^{*} h<^{*} g$ e $h$ é um interpolador do pré-gap $\langle\{f\},\{g\}\rangle$.

O método de "construção de segmentos" que apresentamos para o caso finito resolve, desde que levemente aperfeiçoado, os casos em que apenas uma das famílias $\mathcal{A}$, $\mathcal{B}$ é infinita e mesmo o caso em que ambas as famílias são infinitas. Descreveremos em seguida apenas este último, sendo os outros dois inteiramente análogos e até mesmo mais simples.

Seja $\left\langle\left\{f_{n}: n<\omega\right\},\left\{g_{n}: n<\omega\right\}\right\rangle$ um pré-gap em $\left\langle{ }^{\omega} \omega,<^{*}\right\rangle$; vale então que

$$
f_{0}<^{*} f_{1}<^{*} f_{2}<^{*} \ldots<^{*} f_{n}<^{*} \ldots<^{*} g_{n}<^{*} \ldots<^{*} g_{2}<^{*} g_{1}<^{*} g_{0}
$$


Temos $\left\{n<\omega: g_{0}(n)<f_{0}(n)\right\}$ finito e é possível construir um segmento inicial finito e não-vazio $N_{0}$ de $\omega$ que contenha esse conjunto. Construímos então segmentos finitos, disjuntos, contíguos e não-vazios $N_{1}, N_{2} \subseteq \omega$ tais que $N_{0} \cup N_{1} \cup N_{2}$ é um segmento inicial de $\omega$, os conjuntos finitos $\left\{n<\omega: f_{1}(n)<f_{0}(n)\right\},\left\{n<\omega: g_{0}(n)<g_{1}(n)\right\}$ e $\left\{n<\omega: g_{1}(n)<f_{1}(n)\right\}$ estão contidos em $N_{0} \cup N_{1} \cup N_{2}$ e existam $n_{i} \in N_{i}$ para $i=1,2$ satisfazendo $f_{0}\left(n_{i}\right)<g_{0}\left(n_{i}\right)$. É imediato que em $N_{1} \cup N_{2}$ tem-se $f_{0}(n) \leqslant g_{0}(n)$ para todo $n$, e note que para $\omega \backslash\left(N_{0} \cup N_{1} \cup N_{2}\right)$ já temos $f_{0}(n) \leqslant f_{1}(n) \leqslant g_{1}(n) \leqslant g_{0}(n)$ para todo $n$. Procedendo recursivamente, obtemos uma família $\left\{N_{m}: m<\omega\right\}$ de segmentos disjuntos, contíguos, finitos e não-vazios e tais que

(1) $\bigcup_{m<\omega} N_{m}=\omega$ e, para cada $k<\omega, \bigcup_{m \leqslant k} N_{m}$ é segmento inicial de $\omega$;

(2) Para todo $k<\omega$ impar, digamos $k=2 j+1$, vale para todo $n \in N_{k} \cup N_{k+1}$ que $f_{0}(n) \leqslant f_{1}(n) \leqslant \ldots f_{j}(n) \leqslant g_{j}(n) \leqslant \ldots g_{1}(n) \leqslant g_{0}(n)$, existem $n_{i} \in N_{i}$ satisfazendo $f_{j}\left(n_{i}\right)<g_{j}\left(n_{i}\right)$ para $i=k, k+1$ e $\left\{n<\omega: f_{j+1}(n)<f_{j}(n)\right\},\left\{n<\omega: g_{j}(n)<g_{j+1}(n)\right\}$, $\left\{n<\omega: g_{j+1}(n)<f_{j+1}(n)\right\} \subseteq \bigcup_{m \leqslant k+1} N_{m}$.

Definimos agora $h \in{ }^{\omega} \omega$ pondo

$$
h(n)= \begin{cases}0 & \text { se } n \in N_{0} \\ f_{0}(n) & \text { se } n \in N_{1} \\ g_{0}(n) & \text { se } n \in N_{2} \\ \vdots & \\ f_{j}(n) & \text { se } n \in N_{2 j+1} \\ g_{j}(n) & \text { se } n \in N_{2(j+1)} \text { para cada } j<\omega\end{cases}
$$

É fácil agora ver que $f_{k}<^{*} h<^{*} g_{k}$ para todo $k<\omega$, com argumentos semelhantes aos usados no caso finito e usando fortemente a condição (2) acima; daí, $h$ é interpolador do pré-gap $\left\langle\left\{f_{n}: n<\omega\right\},\left\{g_{n}: n<\omega\right\}\right\rangle$ e mostramos o desejado.

Dissemos que o teorema 3.1 foi uma adaptação do teorema original de Hausdorff principalmente devido ao fato de que o resultado em questão ( Erster Einschaltungssat$z$ ”, p.244 de [Hau36] ) foi enunciado não para funções de $\omega$ em $\omega$, mas para o que se convencionava chamar na época de sequências diádicas(sequências de zeros e uns), e que 
neste trabalho, evidentemente, serão encaradas como elementos de ${ }^{\omega_{2}}$. A estrutura de ordem na qual o teorema foi enunciado originalmente foi, portanto, a estrutura $\left\langle{ }^{\omega} 2,<^{*}\right\rangle$, onde $\left\langle{ }^{\omega} 2, \leqslant^{*}\right\rangle$ é a (pré-)ordem induzida por $\left\langle{ }^{\omega} \omega, \leqslant^{*}\right\rangle$. Podemos então nos perguntar qual é a relação existente entre os problemas de existência de gaps nas estruturas $\left\langle{ }^{\omega} \omega,<^{*}\right\rangle$, $\left\langle{ }^{\omega} 2,<^{*}\right\rangle$ e $\left\langle\mathcal{P}(\omega), \subset^{*}\right\rangle$. Felizmente, o próximo resultado, devido a Rothberger ([Rot41]) nos permite passar de uma estrutura para outra sem maiores problemas.

\section{Proposição 3.2 [ Rothberger ]}

Sejam $\kappa, \lambda$ cardinais regulares.

São equivalentes as afirmações:

(i) Existe um $\left\langle\kappa, \lambda^{*}\right\rangle$-gap em $\left\langle{ }^{\omega} \omega,<^{*}\right\rangle$.

(ii) Existe um $\left\langle\kappa, \lambda^{*}\right\rangle$-gap em $\left\langle{ }^{\omega} 2,<^{*}\right\rangle$.

(iii) Existe um $\left\langle\kappa, \lambda^{*}\right\rangle$-gap em $\left\langle\mathcal{P}(\omega), \subset^{*}\right\rangle$.

Demonstração $($ ii $) \Leftrightarrow$ (iii) Sendo $\Phi$ a "correspondência natural" entre $\mathcal{P}(\omega)$ e ${ }^{\omega_{2}} 2$, isto é, $\Phi: \mathcal{P}(\omega) \mapsto^{\omega} 2$ dada por $\Phi(A)=f_{A}$ onde $f_{A}$ é a função característica do subconjunto $A$ de $\omega$, sabemos que $\Phi$ é bijetora e basta observar que $A \backslash B=\left\{n<\omega: f_{B}(n)<f_{A}(n)\right\}$ $\operatorname{logo} A \subseteq^{*} B \Leftrightarrow f_{A} \leqslant^{*} f_{B}$ e $\Phi$ é um isomorfismo entre as pré-ordens $\subseteq^{*} \mathrm{e} \leqslant$. Assim, as estruturas $\left\langle{ }^{\omega} 2,<^{*}\right\rangle$ e $\left\langle\mathcal{P}(\omega), \subset^{*}\right\rangle$ são isomorfas e $(i i) \Leftrightarrow($ iii $)$ vale.

(ii) $\Rightarrow($ i $)$ : Basta mostrar que um $\left\langle\kappa, \lambda^{*}\right\rangle$-gap em $\left\langle{ }^{\omega} 2,<^{*}\right\rangle$ constitui também um $\left\langle\kappa, \lambda^{*}\right\rangle$ gap em $\left\langle{ }^{\omega} \omega,<^{*}\right\rangle$. Suponhamos então por absurdo que existam $\mathcal{A}, \mathcal{B} \subseteq{ }^{\omega} 2 \subseteq{ }^{\omega} \omega$ tais que $\langle\mathcal{A}, \mathcal{B}\rangle$ é um gap em $\left\langle{ }^{\omega} 2,<^{*}\right\rangle$ mas que exista $h \in{ }^{\omega} \omega$ que interpole o pré-gap $\langle\mathcal{A}, \mathcal{B}\rangle$ em $\left\langle{ }^{\omega} \omega,<^{*}\right\rangle$. Definimos então $\hat{h} \in{ }^{\omega} 2$ pondo

$$
\hat{h}(n)= \begin{cases}h(n) & \text { se } h(n) \in 2 \\ 1 & \text { caso contrário }\end{cases}
$$

Verificações rotineiras nos dão que $\hat{h}$ é interpolador de $\langle\mathcal{A}, \mathcal{B}\rangle$ em $\left\langle{ }^{w} 2,<^{*}\right\rangle$, o que é uma contradição.

(i) $\Rightarrow($ ii): Para essa implicação, Rothberger teve que que trabalhar com sequências diádicas duplas, que era o nome com o qual ele designava as funções de $\omega \times \omega$ em $2=$ $\{0,1\}$. Vamos fixar algumas notações. Se $x \in{ }^{\omega \times \omega} 2$, escreveremos $x=\left\langle x_{m, n}\right\rangle_{m, n<\omega}$, onde $x_{m, n}=x(\langle m, n\rangle)$. Sobre ${ }^{\omega \times \omega} 2$ definimos uma pré-ordem $\leqslant *$ naturalmente, colocando 


$$
x \leqslant^{*} y \Longleftrightarrow\left\{\langle m, n\rangle \in \omega \times \omega: y_{m, n}<x_{m, n}\right\} \text { é finito }
$$

Uma "sequência diádica dupla" $\left\langle x_{m, n}\right\rangle_{m, n<\omega}$ será dita uma seqüência regular se, para qualquer $m<\omega$, valerem as condições

(1) $x_{m, n} \geqslant x_{m, p}$ se $n<p$

(2) $\lim _{n \rightarrow \infty} x_{m, n}=0$

Note então que, se $x$ é seqüência regular, tem-se

(3) $x_{m, n}=\max \left\{x_{m, i}: i \geqslant n\right\}$ para quaisquer $m, n<\omega$.

Denotaremos por $\left({ }^{\omega \times \omega} 2\right)_{r}$ o conjunto das seqüências regulares. Enunciamos agora o seguinte

Lema 3.1 Sejam $\kappa, \lambda$ cardinais regulares. Então todo $\left\langle\kappa, \lambda^{*}\right\rangle$-gap em $\left\langle(\omega \times \omega 2)_{r},<^{*}\right\rangle$ determina um $\left\langle\kappa, \lambda^{*}\right\rangle$-gap em $\left\langle{ }^{\omega \times \omega} 2,<^{*}\right\rangle$. Mais precisamente, se $\mathcal{A}, \mathcal{B} \subseteq\left({ }^{\omega \times} \omega_{2}\right)_{r}$ são tais que $\langle\mathcal{A}, \mathcal{B}\rangle$ é um gap na família das seqüências regulares, então $\langle\mathcal{A}, \mathcal{B}\rangle$ é um gap em $\left\langle{ }^{\omega \times \omega^{\omega}} 2,<^{*}\right\rangle$.

Prova do Lema: Sejam $\kappa, \lambda$ cardinais regulares e $\mathcal{A}=\left\{a_{\alpha}: \alpha<\kappa\right\}, \mathcal{B}=\left\{b_{\beta}: \beta<\right.$ $\lambda\} \subseteq\left({ }^{\omega \times \omega} 2\right)_{r}$ e tais que $\langle\mathcal{A}, \mathcal{B}\rangle$ é um $\left\langle\kappa, \lambda^{*}\right\rangle$-gap na família das seqüências regulares. Suponhamos por absurdo que exista $x=\left\langle x_{m, n}\right\rangle_{m, n<\omega}$ que interpole o pré-gap $\langle\mathcal{A}, \mathcal{B}\rangle$ em $\left\langle{ }^{\omega \times \omega} 2,<^{*}\right\rangle$. Definimos então uma seqüência $y=\left\langle y_{m, n}\right\rangle_{m, n<\omega} \in{ }^{\omega \times \omega} 2$ pondo

$$
y_{m, n}=\max \left\{x_{m, i}: i \geqslant n\right\} \text { para quaisquer } m, n<\omega .
$$

Temos $x \leqslant \leqslant^{*} y$ claramente, já que, de fato, $x \leqslant y$. Além disso, para qualquer seqüência regular $b \in \mathcal{B}$ tem-se $x<^{*} b$, assim a definição de $y$ implica que $y$ é seqüência regular. Mais ainda, podemos afirmar que se $b \in \mathcal{B}$ então $y \leqslant \leqslant^{*} b$ e portanto $y$ é um interpolador de $\langle\mathcal{A}, \mathcal{B}\rangle$ em $\left\langle\left({ }^{\omega \times \omega_{2}} 2\right)_{r},<^{*}\right\rangle$ (pois nesse caso teríamos, para $\alpha<\kappa$ e $\beta<\lambda$ quaisquer, $\left.a_{\alpha}<^{*} x \leqslant \leqslant^{*} y \leqslant b_{\beta+1}<^{*} b_{\beta}\right)$ e disto já teríamos uma contradição com a hipótese de $\langle\mathcal{A}, \mathcal{B}\rangle$ ser um gap em $\left\langle\left({ }^{\omega \times \omega} 2\right)_{r},<^{*}\right\rangle$. De fato: $\operatorname{seja} b=\left\langle b_{m, n}\right\rangle_{m, n<\omega}$ uma seqüência regular qualquer de $\mathcal{B}$. Como $x<^{*} b$, temos que $\left\{\langle m, n\rangle \in \omega \times \omega: b_{m, n}<x_{m, n}\right\}$ é finito, logo existe $m_{0}<\omega$ tal que $x_{m, n} \leqslant b_{m, n}$ para $m \geqslant m_{0}$ e $n$ qualquer. Mas, $b$ é seqüência regular, logo (3) implica que, para $m \geqslant m_{0}$ e $n$ arbitrário tem-se 


$$
y_{m, n}=\max \left\{x_{m, i}: i \geqslant n\right\} \leqslant \max \left\{b_{m, i}: i \geqslant n\right\}=b_{m, n}
$$

Já para $m<m_{0}$, o fato de $y$ e $b$ serem sequências regulares implica que $y_{m, n}=$ $b_{m, n}=0$ para todos exceto no máximo finitos pares $\langle m, n\rangle$ com $m<m_{0}$ e $n<\omega$. Assim, $\left\{\langle m, n\rangle: b_{m, n}<y_{m, n}\right\}$ é finito e $y \leqslant^{*} b$, portanto conseguimos a contradição desejada.

Com o Lema 3.1, a implicação que desejamos segue. Definimos uma aplicação $\Phi:{ }^{\omega} \omega \mapsto\left({ }^{\omega \times \omega_{2}}\right)_{r}$ pondo $\Phi(f)=x_{f}$, onde, para quaisquer $m, n<\omega$,

$$
x_{f}(\langle m, n\rangle)= \begin{cases}0 & \text { se } n \geqslant f(m) \\ 1 & \text { caso contrário }\end{cases}
$$

A aplicação $\Phi$ está bem definida e é claramente injetora; quanto à sobrejetividade, basta observar que as condições (1) e (2) que caracterizam uma sequência regular fazem com que, fixados y seqüência regular e $m<\omega$, exista um certo número natural $n_{m}$ tal que $y_{m, n}=0$ para todo $n \geqslant n_{m}$ e $n_{m}$ é mínimo para essa propriedade. A partir daí, é fácil definir $f \in{ }^{\omega} \omega$ tal que, para cada $m<\omega, f(m)=n_{m}$ e tem-se $x_{f}=y$. É fácil verificar também que $\left[f \leqslant^{*} g \Leftrightarrow x_{f} \leqslant^{*} x_{g}\right.$ ] (e essa é uma equivalência extremamente análoga à equivalência " $f \leqslant \leqslant^{*} g \Leftrightarrow L_{f} \subseteq^{*} L_{g}$ " que usamos na prova do teorema 2.2$)$ e portanto $\Phi$ é um isomorfismo entre $\left\langle{ }^{\omega} \omega, \leqslant^{*}\right\rangle$ e $\left\langle\left({ }^{\omega \times} \times \omega_{2}\right)_{r}, \leqslant^{*}\right\rangle$. Assim, um $\left\langle\kappa, \lambda^{*}\right\rangle$-gap em $\left\langle{ }^{\omega} \omega,<^{*}\right\rangle$ induz naturalmente um $\left\langle\kappa, \lambda^{*}\right\rangle$-gap em $\left\langle\left(\omega \times \omega_{2}\right)_{r},<^{*}\right\rangle$. O Lema 3.1 garante então a existência de um gap em $\left\langle{ }^{\omega \times \omega} 2,<^{*}\right\rangle$. Seja então $\left\langle\left\{x_{\alpha}: \alpha<\kappa\right\},\left\{y_{\beta}: \beta<\lambda\right\}\right\rangle$ esse gap em $\left\langle{ }^{\omega \times \omega} 2,<^{*}\right\rangle$. Basta agora tomar uma bijeção qualquer $h: \omega \times \omega \mapsto \omega \mathrm{e}$, definindo $x_{\alpha}^{\prime}=x_{\alpha} \circ h^{-1}$, $y_{\beta}^{\prime}=y_{\beta} \circ h^{-1}$ para quaisquer $\alpha<\kappa, \beta<\lambda$, temos que $\left\langle\left\{x_{\alpha}^{\prime}: \alpha<\kappa\right\},\left\{y_{\beta}^{\prime}: \beta<\lambda\right\}\right\rangle$ é um gap em $\left\langle{ }^{\omega} 2,<^{*}\right\rangle$ e $(i) \Rightarrow$ (ii) fica demonstrado.

Veja que já podemos afirmar que não existem $\left\langle\omega, \omega^{*}\right\rangle$-gaps nem em $\left\langle{ }^{\omega} 2,<^{*}\right\rangle$ e nem em $\left\langle\mathcal{P}(\omega), \subset^{*}\right\rangle$ já que quaisquer afirmações sobre existência de gaps nas três estruturas em questão em 3.2 são equivalentes.

Vamos agora observar estes e outros resultados a partir de um ponto de vista mais "moderno", relacionando-os com os pequenos cardinais e com alguns conceitos que já trabalhamos, como as "famílias ortogonais e que não podem ser separadas". Salientamos que demos atenção especial aos clássicos resultados de 3.1 e 3.2 no intuito de caracterizar este texto como uma efetiva introdução ao assunto.

Observemos então as condições da Def. 2.2, que trata dos conceitos de "famílias 
ortogonais" e das "famílias que podem ser separadas". Se $\mathcal{F}, \mathcal{G} \subseteq[\omega]^{\omega}$, definimos a relação $\mathcal{F} \perp \mathcal{G}$ a partir da condição

$$
(\forall F \in \mathcal{F})(\forall G \in \mathcal{G})[|F \cap G|<\omega]
$$

isto é, " $F$ e $G$ são a.d. sempre que $F \in \mathcal{F}$ e $G \in \mathcal{G}$ ". Note que isso nos dá, trabalhando com os complementares dos elementos de $\mathcal{G}$,

$$
(\forall F \in \mathcal{F})(\forall G \in \mathcal{G})[|F \backslash(\omega \backslash G)|<\omega]
$$

As duas condições são, evidentemente, equivalentes. Assim, pode-se dizer que " $\mathcal{F} \perp \mathcal{G}$ se e só se $F \subseteq$ $\subseteq^{*}(\omega \backslash G)$ para quaisquer $F \in \mathcal{F}, G \in \mathcal{G}$ ". Analogamente, a asserção "F e $\mathcal{G}$ podem ser separados", que, pela definição 2.2 é equivalente à existência de um conjunto $S \in[\omega]^{\omega}$ satisfazendo

$$
(\forall F \in \mathcal{F})\left[F \subseteq^{*} S\right] \wedge(\forall G \in \mathcal{G})[|S \cap G|<\omega]
$$

também pode ser tradúzida na forma: existe $S \in[\omega]^{\omega}$ tal que

$$
(\forall F \in \mathcal{F})\left[F \subseteq \subseteq^{*}\right] \wedge(\forall G \in \mathcal{G})\left[S \subseteq^{*}(\omega \backslash G)\right]
$$

Sabemos também que, se $A, B \subseteq[\omega]^{\omega}$ então $A \subset^{*} B$ se e só se $(\omega \backslash B) \subset^{*}(\omega \backslash A)$. Assim, as equivalências entre as condições acima e esta última observação demonstram a seguinte

Proposição 3.3 Sejam $\mathcal{F}, \mathcal{G}$ subfamílias quaisquer de $[\omega]^{\omega}$. São equivalentes as afirmações:

(i) $\mathcal{F} \perp \mathcal{G}, \mathcal{F}$ e $\mathcal{G}$ são bem-ordenadas por $\subset^{*}$ e não podem ser separadas.

(ii) $\langle\mathcal{F},\{\omega \backslash G: G \in \mathcal{G}\}\rangle$ é um gap em $\left\langle\mathcal{P}(\omega), \subset^{*}\right\rangle$.

É importante notarmos que, se duas subfamílias de $[\omega]^{\omega}$ são ortogonais, então cada um de seus elementos tem complementar infinito, isto é, seus elementos são coinfinitos; dessa forma, o item (ii) da Proposição anterior não deve causar nenhuma estranheza.

Segue da Proposição 3.3 que afirmações sobre gaps em $\left\langle\mathcal{P}(\omega), \subset^{*}\right\rangle$ têm sempre "afirmações duais" na linguagem de famílias ortogonais e que não podem ser separadas, e reciprocamente. Por exemplo, a igualdade $\mathfrak{b}=\mathfrak{b}_{6}$ do Teorema 2.2 equivale ao seguinte resultado de Rothberger: 


\section{Teorema 3.2 ( [Rot41], p.121)}

Seja $\lambda$ o menor cardinal infinito para o qual exista um $\left\langle\lambda, \omega^{*}\right\rangle$-gap em $\left\langle\mathcal{P}(\omega), \subset^{*}\right\rangle$. Então $\lambda=\mathfrak{b}$.

Convidamos o leitor a observar que "alguma coisa nesse sentido" já havia sido feita neste trabalho, a saber o Lema 2.1 utilizado na demonstração de " $\mathfrak{p}=\omega_{1} \Leftrightarrow \mathfrak{t}=\omega_{1}$ ", item b) do Teorema 2.1; o papel do cardinal b naquele Lema é fundamental para a construção de uma espécie de "interpolador" entre duas famílias ( a primeira com cardinalidade menor que $\mathfrak{b}$ e a segunda enumerável) que possuíam, "a menos da boa-ordenação", uma estrutura análoga a de um pré-gap.

Note que, como $\mathfrak{b}>\omega$, a Proposição 3.2 e o Teorema 3.2 têm como consequência imediata o fato que enunciamos no Teorema 3.1 para $\left\langle{ }^{\omega} \omega,<^{*}\right\rangle$ e depois passamos para $\left\langle{ }^{\omega} 2,<^{*}\right\rangle$ e $\left\langle\mathcal{P}(\omega), C^{*}\right\rangle$ : a não existência de $\left\langle\omega, \omega^{*}\right\rangle$-gaps em nenhuma dessas três estruturas.

Essa não existência de $\left\langle\omega, \omega^{*}\right\rangle$-gaps sugere uma questão óbvia: existe algum cardinal regular $\kappa$ para o qual exista um gap simétrico, isto é, um $\left\langle\kappa, \kappa^{*}\right\rangle$-gap em alguma das três estruturas, e, conseqüentemente, em todas elas ? E, se existirem gaps simétricos, qual é o menor cardinal regular para o qual isso acontece ? A situação parece análoga às que originaram nossos pequenos cardinais: determinar um cardinal não-enumerável,limitado superiormente por $\mathfrak{c}$ e mínimo para uma determinada propriedade. Porém, o seguinte resultado de Hausdorff nos indica que não há a necessidade de definirmos um novo pequeno cardinal para esse caso.

Teorema 3.3 ( [Hau36] ) Existem $\left\langle\omega_{1}, \omega_{1}^{*}\right\rangle$-gaps.

Os $\left\langle\omega_{1}, \omega_{1}^{*}\right\rangle$-gaps são hoje em dia denominados Hausdorff gaps, ou, em português, gaps de Hausdorff. Demonstraremos o teorema acima na seção seguinte, usando a terminologia de famílias ortogonais que não podem ser separadas ( que é a terminologia com a qual serão construídos alguns exemplos de espaços topológicos, no último capítulo ). Na próxima seção veremos também que estruturas semelhantes a gaps podem surgir em contextos em que não apareçam necessariamente subconjuntos totalmente ordenados de $\left\langle\mathcal{P}(\omega), \subset^{*}\right\rangle$; com efeito, veremos que existe uma família a.d. $\mathcal{A} \subseteq[\omega]^{\omega} \operatorname{com}|\mathcal{A}|=\omega_{1}$ e tal que, se $\mathcal{B}$ e $\mathcal{C}$ são subfamílias disjuntas e não-enumeráveis quaisquer de $\mathcal{A}$, então $\mathcal{B}$ e $\mathcal{C}$ são famílias ortogonais (obviamente!) e que não podem ser separadas.

Com relação ao cardinal $\mathfrak{t}$ e gaps, iniciemos com a seguinte 
Proposição 3.4 Seja $\kappa$ um cardinal infinito. São equivalentes as afirmações:

(i) Existe um $\left\langle\kappa, 1^{*}\right\rangle$-gap em $\left\langle\mathcal{P}(\omega), \subset^{*}\right\rangle$.

(ii) Existe um $\left\langle 0, \kappa^{*}\right\rangle$-gap em $\left\langle[\omega]^{\omega}, \subset^{*}\right\rangle$.

Demonstração $(i) \Rightarrow$ (ii) Seja $\left\langle\left\{A_{\alpha}: \alpha<\kappa\right\},\{B\}\right\rangle$ um $\left\langle\kappa, 1^{*}\right\rangle$-gap em $\left\langle\mathcal{P}(\omega), C^{*}\right\rangle$. Temos, para $\alpha<\beta<\kappa$ quaisquer, $A_{\alpha} \subset^{*} A_{\beta} \subset^{*} B$ e não existe $X \subseteq \omega$ tal que $A_{\alpha} \subset^{*} X \subset^{*} B$ para todo $\alpha<\kappa$. Temos então, para $\alpha<\beta<\kappa, B \backslash A_{\beta} \subset^{*} B \backslash A_{\alpha}$ (pois $\left(B \backslash A_{\beta}\right) \backslash\left(B \backslash A_{\alpha}\right)=B \cap\left(A_{\alpha} \backslash A_{\beta}\right)$, que é finito já que $A_{\alpha} \backslash A_{\beta}$ é finito, e $\left(B \backslash A_{\alpha}\right) \backslash\left(B \backslash A_{\beta}\right)=B \cap\left(A_{\beta} \backslash A_{\alpha}\right)$ que é infinito já que $A_{\beta} \backslash A_{\alpha}$ é infinito e $A_{\beta} \backslash B$ é finito). Daí temos $\left\{B \backslash A_{\alpha}: \alpha<\kappa\right\} \subseteq[\omega]^{\omega}$ e bem-ordenado por $\supset^{*}$. Afirmamos agora que não pode existir $X \in[\omega]^{\omega}$ tal que $X \subseteq^{*} B \backslash A_{\alpha}$ para todo $\alpha<\kappa$, pois nesse caso teríamos $A_{\alpha} \subseteq^{*} B \backslash X \subset^{*} B$ para todo $\alpha<\kappa$, o que é uma contradição. De fato: se, para cada $\alpha<\kappa, X \backslash\left(B \backslash A_{\alpha}\right)$ fosse finito teríamos $X \backslash B$ e $X \cap A_{\alpha}$ finitos, donde $A_{\alpha} \backslash(B \backslash X)=\left(A_{\alpha} \backslash B\right) \cup\left(A_{\alpha} \cap X\right)$ seria finito e $B \backslash(B \backslash X)=B \cap X$ seria infinito.

$($ ii $) \Rightarrow(i)$ : Seja $\left\langle\emptyset,\left\{\bar{B}_{\alpha}: \alpha<\kappa\right\}\right\rangle$ um $\left\langle 0, \kappa^{*}\right\rangle$-gap em $\left\langle[\omega]^{\omega}, \subset^{*}\right\rangle$. Temos então que $\left\{B_{\alpha}: \alpha<\kappa\right\}$ é uma torre, já que é um conjunto bem-ordenado por $\supset^{*}$ e que não possui pseudo-intersecção infinita (pois seria exatamente uma pseudo-intersecção infinita de $\left\{B_{\alpha}: \alpha<\kappa\right\}$ o "candidato" a interpolador do pré-gap $\left\langle\emptyset,\left\{B_{\alpha}: \alpha<\kappa\right\}\right\rangle$ em $\left\langle[\omega]^{\omega}, \subset^{*}\right\rangle$; note que colocamos $\left\langle[\omega]^{\omega}, \subset^{*}\right\rangle$ ao invés de $\left\langle\mathcal{P}(\omega), \subset^{*}\right\rangle$ pois pseudo-intersecções finitas são triviais.). Passando ao complementar, temos que $\left\{\omega \backslash B_{\alpha}: \alpha<\kappa\right\}$ é uma subfamília de $\mathcal{P}(\omega)$ que está bem-ordenada por $\subset^{*}$. Afirmamos que $\left\langle\left\{\omega \backslash B_{\alpha}: \alpha<\kappa\right\},\{\omega\}\right\rangle$ é um $\left\langle\kappa, 1^{*}\right\rangle$-gap em $\left\langle\mathcal{P}(\omega), \subset^{*}\right\rangle$. De fato, suponha que exista $X \subseteq \omega$ tal que $\omega \backslash B_{\alpha} \subseteq^{*} X \subset^{*} \omega$ para todo $\alpha<\kappa$. Daí $\omega \backslash X$ seria pseudo-intersecção infinita da torre $\left\{B_{\alpha}: \alpha<\kappa\right\}$, contradição.

Ficou implícita na demonstração de $(i) \Rightarrow($ ii $)$ da proposição anterior o fato de que uma torre de tipo de ordem $\zeta$ é um $\left\langle 0, \zeta^{*}\right\rangle$-gap em $\left\langle[\omega]^{\omega}, \subset^{*}\right\rangle$. A equivalência entre as condições $(i)$ e (ii) apresentadas nos permite então escrever

$$
\mathfrak{t}=\min \left\{\lambda: \lambda \text { é cardinal infinito e existe um }\left\langle\lambda, 1^{*}\right\rangle \text {-gap em }\left\langle\mathcal{P}(\omega), \subset^{*}\right\rangle\right\}
$$

Note que podemos restringir a questão somente a cardinais infinitos, usando a demonstração do Teorema 3.1 e a Proposição 3.2 . 


\subsection{O cardinal $\omega_{1}$ e os gaps de Luzin e de Hausdorff}

A proposição 3.2 registrou o fato de que $\mathfrak{b}$ é o menor cardinal $\lambda$ para o qual existe um $\left\langle\lambda, \omega^{*}\right\rangle$-gap em $\left\langle\mathcal{P}(\omega), C^{*}\right\rangle$; destacamos que esse fato é conseqüência imediata da igualdade $\mathfrak{b}=\mathfrak{b}_{6}$ do Teorema 2.2 e das equivalências entre afirmações sobre gaps e famílias ortogonais, bem-ordenadas por $C^{*}$ e que não podem ser separadas, conforme enunciado na Proposição 3.3 , já que a igualdade $\mathfrak{b}=\mathfrak{b}_{6}$ diz originalmente que $\mathfrak{b}$ é a menor cardinalidade de uma subfamília $\mathcal{B}$ de $[\omega]^{\omega}$ para a qual exista $\mathcal{C} \subseteq[\omega]^{\omega} \operatorname{com}|\mathcal{C}|=\omega, \mathcal{B} \perp \mathcal{C}$, $\mathcal{B}$ e $\mathcal{C}$ bem-ordenados por $\subseteq^{*}$ e $\mathcal{B}, \mathcal{C}$ não podendo ser separados. Já a Observação 2.2, feita logo após a demonstração do Teorema 2.2 , destaca o fato de que $\mathfrak{b}$ é a menor cardinalidade de uma subfamília $\mathcal{B}$ de $[\omega]^{\omega}$ para a qual existe $\mathcal{C} \subseteq[\omega]^{\omega}$ com $|\mathcal{C}|=\omega, \mathcal{B} \cap \mathcal{C}=\emptyset, \mathcal{B} \cup \mathcal{C}$ a.d. e $\mathcal{B}, \mathcal{C}$ não podendo ser separados. Uma questão natural a se levantar seria: qual é a menor cardinalidade de $\mathcal{B}$ se trocarmos " $|\mathcal{C}|=\omega$ " por " $|\mathcal{C}|=\omega_{1}$ " em cada um dos dois casos descritos neste parágrafo?

É claro que essa cardinalidade mínima é $\omega$ se e só se $\mathfrak{b}=\omega_{1}$; basta "trocar os papéis" de $\mathcal{B}$ e $\mathcal{C}$ nos enunciados e lembrar que a relação "podem ser separados" é simétrica. O que veremos nesta seção é que, se $\mathfrak{b}>\omega_{1}$, então novamente a cardinalidade mínima para os dois casos coincidirá e nesse caso será igual a $\omega_{1}$. Isso será feito demonstrando-se a existência dos "gaps de Luzin" e dos gaps de Hausdorff, notáveis resultados provados em $Z F C$ e que, dessa forma, valem independentemente de qual seja o valor de $\mathfrak{b}$, ou mesmo de $\boldsymbol{c}$.

\section{Teorema 3.4 [Existência dos "gaps de Luzin"]}

Existem subfamílias disjuntas $\mathcal{B}, \mathcal{C} \subseteq[\omega]^{\omega}$ satisfazendo $|\mathcal{B}|=|\mathcal{C}|=\omega_{1}$ e tais que $\mathcal{B} \cup \mathcal{C}$ é a.d. mas $\mathcal{B}$ e $\mathcal{C}$ não podem ser separados. De fato, existe $\mathcal{A} \subseteq[\omega]^{\omega}$ tal que $|\mathcal{A}|=\omega_{1}$ $e \mathcal{A}$ é a.d. mas, para quaisquer $\mathcal{B}, \mathcal{C} \subseteq \mathcal{A}$ disjuntos e não-enumeráveis, $\mathcal{B}$ e $\mathcal{C}$ não podem ser separados.

Note que estamos escrevendo "gap de Luzin", com aspas, para designar uma estrutura como a descrita acima, para enfatizar que, conforme comentamos no final da seção anterior, tal estrutura não provém de subconjuntos totalmente ordenados por $\subset^{*}$.

Demonstração Iremos construir por recursão transfinita uma família $\mathcal{A}=\left\{A_{\zeta}: \zeta<\right.$ 
$\left.\omega_{1}\right\} \subseteq[\omega]^{\omega}$ satisfazendo para cada $\zeta<\omega_{1}$

(1) $(\forall \xi<\zeta)\left[\left|A_{\xi} \cap A_{\zeta}\right|<\omega\right]$

(2) $(\forall k<\omega)\left[\left|\left\{\xi<\zeta: \sup \left(A_{\xi} \cap A_{\zeta}\right)<k\right\}\right|<\omega\right]$

e após verificarmos que essa construção está bem definida mostraremos que tal família satisfaz o desejado.

Pois bem. Inicialmente, enumeramos na forma $\left\{A_{k}: k<\omega\right\}$ uma subfamília disjunta qualquer de $[\omega]^{\omega}$. É claro que (1) e (2) valem para cada $k<\omega$. Seja agora $\omega \leqslant \zeta<\omega_{1}$ e assuma que $\left\{A_{\xi}: \xi<\zeta\right\}$ esteja bem construída. Reenumeramos $\left\{A_{\xi}: \xi<\zeta\right\}$ na forma $\left\{Z_{n}: n<\omega\right\}$. Então, dado $n<\omega$ qualquer,

$$
Z_{n} \backslash \bigcup_{k<n} Z_{k}=Z_{n} \backslash\left(\bigcup_{k<n}\left(Z_{n} \cap Z_{k}\right)\right)
$$

é infinito e portanto existe uma aplicação $a: \omega \mapsto \omega$ satisfazendo

$$
(\forall n<\omega)\left[a(n) \in\left(Z_{n} \backslash \bigcup_{k<n} Z_{k}\right) \backslash n\right]
$$

Tome $A_{\zeta}=i m(a)$. Temos $A_{\zeta}$ claramente infinito e é imediato que

$$
(\forall n<\omega)\left[\left(\left|Z_{n} \cap A_{\zeta}\right| \leqslant n+1\right) \wedge\left(\sup \left(Z_{n} \cap A_{\zeta}\right) \geqslant a_{n} \geqslant n\right)\right]
$$

donde (1) e (2) valem para $A_{\zeta}$ e a recursão está bem definida. $|\mathcal{A}|=\omega_{1}$ e $\mathcal{A}$ é a.d., logo resta verificar o que ocorre para $\mathcal{B}, \mathcal{C} \subseteq \mathcal{A}$ disjuntos e não-enumeráveis.

Sejam então $B, C \in\left[\omega_{1}\right]^{\omega_{1}}$ disjuntos e $S \subseteq \omega$ qualquer; mostraremos que $S$ não separa $\left\{A_{\xi}: \xi \in B\right\}$ e $\left\{A_{\zeta}: \zeta \in C\right\}$. Com efeito, mostraremos que se vale que $(\forall \zeta \in C)\left[\left|S \cap A_{\zeta}\right|<\omega\right]$, então $(\exists \xi \in B)\left[A_{\xi} \nsubseteq^{*} S\right]$.

De fato: se vale a condição assumida para $C$ no parágrafo anterior, podemos escrever

$$
C=\bigcup_{m<\omega}\left\{\zeta \in C: \sup \left(S \cap A_{\zeta}\right) \leqslant m\right\}
$$

e é fácil ver que existe um determinado $m<\omega$ para o qual

$$
H=\left\{\zeta \in C: \sup \left(S \cap A_{\zeta}\right)=m\right\}
$$


é infinito (aliás, não-enumerável). Agora, como $|B|=\omega_{1}$, obrigatoriamente $\sup (B)=\omega_{1}$ e existe $\xi \in B$ para o qual $H \cap \xi$ é infinito. Temos então de (2) que para cada $k<\omega$ existe $\alpha_{k} \in H \cap \xi$ tal que $a_{\alpha_{k}}=\sup \left(A_{\alpha_{k}} \cap A_{\xi}\right) \geqslant k$. Segue que $\left|A_{\xi} \backslash S\right|=\omega$, pois para todo $j>m$ temos $m<j \leqslant a_{\alpha} \notin S$ e portanto $\left\{a_{\alpha_{j}}: j>m\right\}$ é um subconjunto infinito de $A_{\xi} \backslash S$. Assim, $\mathcal{A}$ satisfaz o desejado.

Observe que, na demonstração que acabamos de fazer, bastava saber que a condição (2) da construção valia para os $\xi$ 's de B; esse "enfraquecimento de exigência" será útil em breve.

Do Teorema 3.4 e da Observação 2.2, segue o

Corolário $\omega_{1}=\min \left\{|\mathcal{B}|: \mathcal{B} \subseteq[\omega]^{\omega}\right.$ é a.d. e $(\exists \mathcal{C} \subseteq \mathcal{B})[\mathcal{C}$ e $\mathcal{B} \backslash \mathcal{C}$ não podem ser separados ]\}

Demonstração Seja $\lambda$ o cardinal definido pela expressão à direita; o Teorema 3.4 nos dá $\lambda \leqslant \omega_{1}$. Note que $\mathcal{C}$ como na definição de $\lambda$ deve ser infinito ( pois, se $\mathcal{C}=\emptyset$, qualquer subconjunto finito o separa de qualquer outra família, e $\emptyset \neq \mathcal{C}$ finito pode ser separado de $\mathcal{B} \backslash \mathcal{C}$ por $S=\omega \backslash \bigcup \mathcal{C})$. Assim, $\lambda=\omega$ ou $\lambda=\omega_{1}$. Mas, não podemos ter $\lambda=\omega$ devido à Observação 2.2 , já que $\mathfrak{b}>\omega$. Portanto, $\lambda=\omega_{1}$.

Apresentaremos agora o principal resultado deste capítulo: a demonstração de que existem gaps de Hausdorff, $\left\langle\omega_{1}, \omega_{1}^{*}\right\rangle$-gaps em $\left\langle\mathcal{P}(\omega), \subset^{*}\right\rangle$, existência essa já "anunciada" (sem demonstração) no Teorema 3.3. Segue o enunciado na linguagem de "famílias ortogonais, bem-ordenadas por $\subset^{*}$ e que não podem ser separadas".

\section{Teorema 3.5 (Existência dos Gaps de Hausdorff)}

Existem famílias $\mathcal{A}, \mathcal{B} \subseteq[\omega]^{\omega}$, ambas bem-ordenadas por $\subset^{*}$ com tipo de ordem $\omega_{1}$, tais que $\mathcal{A} \perp \mathcal{B}$ e $\mathcal{A}$ e $\mathcal{B}$ não podem ser separadas.

Antes de iniciarmos a demonstração do teorema, vamos introduzir o conceito de "estar perto de", que foi o "truque" que Hausdorff usou para construir o seu gap. Sejam $X \subseteq \omega$ e $\mathcal{A} \subseteq \mathcal{P}(\omega)$; diremos que " $X$ está perto de $\mathcal{A}$ ", e indicaremos isso por $X(p) \mathcal{A}$, se para todo $n<\omega$ o conjunto $\{A \in \mathcal{A}: A \cap X \subseteq n\}$ for finito. Note que, com essa definição, poderíamos escrever a exigência (2) do teorema anterior da seguinte maneira: 
" $A_{\zeta}(p)\left\{A_{\xi}: \xi<\zeta\right\}$ para todo $\zeta<\omega_{1}$ ". Essa semelhança "não é mera coincidência", pois na verdade o resultado de Luzin surgiu poucos anos depois do resultado de Hausdorff.

São de fácil verificação as seguintes asserções, para $X, Y \subseteq \omega$ e $\mathcal{A}, \mathcal{B} \subseteq \mathcal{P}(\omega)$ quaisquer:

$$
\begin{aligned}
& (P 1) X(p) \mathcal{A}, X \subseteq \subseteq^{*} Y \Rightarrow Y(p) \mathcal{A} \\
& (P 2) X(p) \mathcal{A}, \mathcal{B} \subseteq \mathcal{A} \Rightarrow X(p) \mathcal{B}
\end{aligned}
$$

Demonstração Construiremos simultaneamente, por recursão em $\zeta<\omega_{1}, \mathcal{A}=\left\{A_{\zeta}\right.$ : $\left.\zeta<\omega_{1}\right\}, \mathcal{B}=\left\{B_{\zeta}: \zeta<\omega_{1}\right\} \subseteq[\omega]^{\omega}$ satisfazendo para cada $\zeta<\omega_{1}$

(1) $\left|\omega \backslash\left(A_{\zeta} \cup B_{\zeta}\right)\right|=\omega$ (isto é, $A_{\zeta} \cup B_{\zeta}$ é coinfinito)

(2) $A_{\zeta} \cap B_{\zeta}=\emptyset$

(3) $(\forall \xi<\zeta)\left[\left(A_{\xi} \subset^{*} A_{\zeta}\right) \wedge\left(B_{\xi} \subset^{*} B_{\zeta}\right)\right]$

(4) $A_{\zeta}(p)\left\{B_{\xi}: \xi<\zeta\right\}$

Note que a condição (4) acima corresponde ao "enfraquecimento de exigência" de que tratamos logo após a demonstração do teorema 3.4. Porém, ainda assim a construção de Hausdorff é mais elaborada que a de Luzin.

A verificação da recursão acima é como segue:

$\zeta=0$ : Particionamos $\omega$ em três conjuntos infinitos e disjuntos $A, B$ e $C$ e pomos $A_{0}=A, B_{0}=B$. (1) e (2) são óbvias e (3) e (4) valem por vacuidade.

$\zeta$ ordinal sucessor: Seja $\zeta=\delta+1$ e suponhamos $\left\{A_{\xi}: \xi \leqslant \delta\right\},\left\{B_{\xi}: \xi \leqslant \delta\right\}$ bem construídas. Particionamos $\omega \backslash\left(A_{\delta} \cup B_{\delta}\right)$ em três conjuntos infinitos e disjuntos $D, E$ e $F$ e pomos $A_{\zeta}=A_{\delta} \cup D, B_{\zeta}=B_{\delta} \cup E$. (1), (2) e (3) valem obviamente, e para (4) note que $(P 1)$ dá $A_{\zeta}(p)\left\{B_{\xi}: \xi<\delta\right\}$ e daí é imediato que $A_{\zeta}(p)\left\{B_{\xi}: \xi \leqslant \delta\right\}$.

$\zeta$ ordinal limite: Suponha $\left\{A_{\xi}: \xi<\zeta\right\},\left\{B_{\xi}: \xi<\zeta\right\}$ bem construídas. Afirmamos que existem $X \subseteq \omega$ coinfinito e $S \subseteq X$ satisfazendo

(5) $(\forall \xi<\zeta)\left[A_{\xi} \cup B_{\xi} \subseteq^{*} X\right]$

(6) $(\forall \xi<\zeta)\left[\left(A_{\xi} \subseteq^{*} S\right) \wedge\left(\left|S \cap B_{\xi}\right|<\omega\right)\right]$

De fato: (1) e (3) nos dão, passando aos complementares, que $\left\{\omega \backslash\left(A_{\xi} \cup B_{\xi}\right)\right.$ : $\xi<\zeta\} \subseteq[\omega]^{\omega}$ é uma família bem-ordenada por $\supseteq^{*}$; daí $\mathfrak{t}_{1}>\omega=|\zeta|$ nos dá $X^{\prime}$, pseudo- 
intersecção infinita de $\left\{\omega \backslash\left(A_{\xi} \cup B_{\xi}\right): \xi<\zeta\right\}$ e podemos tomar $X=\omega \backslash X^{\prime}$. Já as condições (2) e (3) nos dão $\left\{A_{\xi}: \xi<\zeta\right\} \perp\left\{B_{\xi}: \xi<\zeta\right\}$ (com efeito, se $\xi_{1}$, $\xi_{2}$ são menores que $\zeta, \xi_{1}=\xi_{2}$ implica $A_{\xi_{1}} \cap B_{\xi_{2}}=\emptyset$ e $\xi_{1}<\xi_{2}$ implica $A_{\xi_{1}} \cap B_{\xi_{2}} \subseteq^{*} A_{\xi_{2}} \cap B_{\xi_{2}}=\emptyset$ obtendo-se o desejado, analogamente para $\xi_{2}<\xi_{1}$ ) e essas famílias estão bem-ordenadas por $\subset^{*}$. Agora $\mathfrak{b}_{6}>\omega=|\zeta|$ nos dá que existe um conjunto separador $S^{\prime}$ satisfazendo

$$
(\forall \xi<\zeta)\left[\left(A_{\xi} \subseteq^{*} S^{\prime}\right) \wedge\left(\left|S^{\prime} \cap B_{\xi}\right|<\omega\right)\right]
$$

e verifica-se facilmente que $S=X \cap S^{\prime}$ é um subconjunto de $X$ que satisfaz (6).

Definimos agora, para cada $k<\omega$,

$$
M_{k}=\left\{\xi<\zeta: S \cap B_{\xi} \subseteq k\right\}
$$

e por recursão finita definimos uma seqüência $\left\langle S_{n}: n<\omega\right\rangle$ de subconjuntos de $X$ satisfazendo, para cada $k<\omega$,

(7) $S_{k} \perp\left\{B_{\xi}: \bar{\xi}<\zeta\right\}$

(8) $S_{k} \subseteq S_{k+1}$

(9) $S_{k+1}(p)\left\{B_{\xi}: \xi \in M_{k}\right\}$

Podemos tomar $S_{0}=S$. Supondo agora que $S_{k}$ está bem construído, definiremos $S_{k+1}$. Se $M_{k}$ é finito, posso tomar $S_{k+1}=S_{k}$ e as condições valem obviamente. Suponha então $M_{k}$ infinito: note que, se $\xi<\zeta$, (4) nos dá $A_{\xi}(p)\left\{B_{\alpha}: \alpha<\xi\right\}$ e de $A_{\xi} \subseteq^{*} S$ e $(P 1)$ temos $S(p)\left\{B_{\alpha}: \alpha<\xi\right\}$, donde, em particular,

$$
M_{k} \cap \xi=\left\{\alpha<\xi: S \cap B_{\alpha} \subseteq k\right\}
$$

é finito e portanto $M_{k}$ é cofinal em $\zeta$ e tem tipo de ordem $\omega$. Seja então $b: \omega \mapsto M_{k}$ o isomorfismo de ordem. Temos que para cada $n<\omega$ o conjunto

$$
G_{n}=\left(B_{b(n)} \backslash \bigcup_{i<n} B_{b(i)}\right) \cap X
$$

é infinito, por (3) e (5) ( note que $B_{b(n)} \backslash \bigcup_{i<n} B_{b(i)}={ }^{*} B_{b(n)} \backslash B_{b(n-1)}$ é infinito e $B_{b(n)} \subseteq^{*} X$ garante que a intersecção desse conjunto com $X$ é infinita ) logo posso tomar $p_{n} \in G_{n}$ $\operatorname{com} p_{n} \geqslant n$ para todo $n<\omega$. 
Afirmamos agora que $S_{k+1}=S_{k} \cup\left\{p_{i}: i<\omega\right\} \subseteq X$ satisfaz o desejado. (8) é evidente. $S_{k} \perp\left\{B_{\xi}: \xi<\zeta\right\}$ implica $S_{k+1} \perp\left\{B_{\xi}: \xi<\zeta\right\}$ já que, dado $\xi<\zeta$ qualquer, $\left|\left\{p_{i}: i<\omega\right\} \cap B_{\xi}\right|=\left|\left\{i<\omega: p_{i} \in B_{\xi}\right\}\right|$ e $\left\{i<\omega: p_{i} \in B_{\xi}\right\} \subseteq^{*} m+1$, onde $m=\min \{i<$ $\omega: \xi<b(i)\}$, e portanto temos (7). É claro que $\left\{p_{i}: i<\omega\right\}(p)\left\{B_{\xi}: \xi \in M_{k}\right\}$ já que, dado $n<\omega,\left\{\xi \in M_{k}:\left(\left\{p_{i}: i<\omega\right\} \cap B_{\xi}\right) \subseteq n\right\} \subseteq\{b(0), \ldots, b(n-1)\}$ e assim $(P 1)$ implica $S_{k+1}(p)\left\{B_{\xi}: \xi \in M_{k}\right\}$, portanto temos (9) e a sequência está bem definida.

Temos agora $\left\{S_{n}: n<\omega\right\}$ bem-ordenado por $\subseteq$ e $\left\{S_{n}: n<\omega\right\} \perp\left\{B_{\xi}: \xi<\zeta\right\}$; usando novamente $\mathfrak{b}_{6}>\omega$, obtenho $A_{\zeta}$ tal que

$$
(\forall n<\omega)\left[\left(S_{n} \subseteq^{*} A_{\zeta}\right) \wedge\left(A_{\zeta} \perp\left\{B_{\xi}: \xi<\zeta\right\}\right)\right]
$$

e podemos supor ainda que $S \subseteq A_{\zeta} \subseteq X$ (pois, se A é um conjunto separador para $\left\{S_{n}: n<\omega\right\}$ e $\left\{B_{\xi}: \xi<\zeta\right\}$, então $(A \cap X) \cup S$ também o é.). Definimos então $B_{\zeta}=X \backslash A_{\zeta} . X$ coinfinito implica (1). (2) é óbvio. Para (3), basta notar que, para todo $\xi<\zeta, A_{\xi} \subset^{*} A_{\xi+1} \subseteq^{*} S \subseteq A_{\zeta}$ e $B_{\xi} \subset^{*} B_{\xi+1} \subseteq^{*} B_{\zeta}$ (já que, se $\delta=\xi+1$, então $B_{\delta} \backslash B_{\zeta}=\left(B_{\delta} \backslash X\right) \cup\left(\overline{B_{\delta}} \cap A_{\zeta}\right), \operatorname{logo} B_{\delta} \backslash B_{\zeta}$ é união de dois conjuntos finitos - o primeiro devido a (5) e o segundo devido a $A_{\zeta} \perp\left\{B_{\xi}: \xi<\zeta\right\}$ ). Resta então verificar (4). Suponha então por absurdo que exista um determinado $j<\omega$ para o qual

$$
M^{\prime}=\left\{\xi<\zeta:\left(A_{\zeta} \cap B_{\xi}\right) \subseteq j\right\}
$$

seja infinito. De $S \subseteq A_{\zeta}$ conclui-se que $M^{\prime} \subseteq M_{j}$. Mas $S_{j+1}^{\prime}(p)\left\{B_{\xi}: \xi \in M_{j}\right\}$ e $S_{j+1}^{\prime} \subseteq^{*} A_{\zeta}$, $\operatorname{assim}(P 1)$ nos dá $A_{\zeta}(p)\left\{B_{\xi}: \xi \in M_{j}\right\}$, donde, em particular, $\left\{\xi \in M_{j}:\left(A_{\zeta} \cap B_{\xi}\right) \subseteq j\right\}=$ $M^{\prime} \cap M_{j}$ é finito, o que é uma contradição. Assim, (4) vale e a construção das famílias $\mathcal{A}$ e $\mathcal{B}$ está feita.

Afirmamos agora que $\mathcal{A}=\left\{A_{\zeta}: \zeta<\omega_{1}\right\}$ e $\mathcal{B}=\left\{B_{\zeta}: \zeta<\omega_{1}\right\}$ satisfazem o enunciado. A boa ordenação por $\subset^{*}$ com tipo de ordem $\omega_{1}$ é clara, e $\mathcal{A} \perp \mathcal{B}$ é obtido de modo análogo ao que fizemos no caso " $\zeta$ limite" da recursão; resta ver que $\mathcal{A}$ e $\mathcal{B}$ não podem ser separados. A prova é inteiramente análoga à parte final da demonstração de 3.4(e eis o momento em que o "enfraquecimento de exigência" torna-se útil, pois precisa-se apenas que "um determinado $A_{\zeta}$ esteja perto da família dos $B$ 's com índices menores que $\left.\zeta^{\prime \prime}\right)$ : tomamos $Z$ um conjunto que satisfaça $\left(\forall \zeta<\omega_{1}\right)\left[\left|Z \cap B_{\zeta}\right|<\omega\right]$. Então podemos escrever 


$$
\omega_{1}=\bigcup_{m<\omega}\left\{\zeta<\omega_{1}: Z \cap B_{\zeta} \subseteq m\right\}
$$

e portanto existe $k<\omega$ tal que

$$
H=\left\{\zeta<\omega_{1}: Z \cap B_{\zeta} \subseteq k\right\}
$$

é não-enumerável. Tomo agora $\xi<\omega_{1}$ para o qual $H \cap \xi$ é infinito. De $A_{\xi}(p)\left\{B_{\alpha}: \alpha<\xi\right\}$ temos, para cada $i<\omega,\left\{\alpha<\xi: A_{\xi} \cap B_{\alpha} \subseteq i\right\}$ finito, logo para cada $i<\omega$ existe $\alpha_{i} \in H \cap \xi$ e $a_{\alpha_{i}} \in A_{\xi} \cap B_{\alpha_{i}}$ satisfazendo $a_{\alpha_{i}} \geqslant i$. Segue que $\left|A_{\xi} \backslash Z\right|=\omega$, pois para cada $j>k$ temos $k<j \leqslant a_{\alpha_{j}} \notin Z$ e portanto $\left\{a_{\alpha_{j}}: k<j\right\}$ é um subconjunto infinito de $A_{\xi} \backslash Z$, portanto $A_{\xi} \nsubseteq^{*} Z$ e $\mathcal{A}$ e $\mathcal{B}$ não podem ser separados.

Corolário $\omega_{1}=\min \left\{\kappa: \exists \mathcal{A}, \mathcal{B} \subseteq[\omega]^{\omega} \operatorname{com}|\mathcal{A}|=|\mathcal{B}|=\kappa\right.$ e $\mathcal{A} \perp \mathcal{B}, \mathcal{A}$ e $\mathcal{B}$ são bem-ordenados por $\subset^{*}$ mas não podem ser separados $\}$ $=\min \left\{\kappa:\right.$ existe um $\left\langle\kappa, \kappa^{*}\right\rangle$-gap em $\left.\left\langle\mathcal{P}(\omega), \subset^{*}\right\rangle\right\}$.

Veremos no próximo capítulo que a existência de gaps de Hausdorff que satisfazem a determinadas hipóteses adicionais é equivalente a afirmações do tipo " $\mathfrak{p}=\omega_{1}$ " (existência de "tight gaps") ou "b $=\omega_{1}$ " (existência de "big gaps").

Encerramos o capítulo com uma pequena aplicação topológica dos gaps de Hausdorff.

Proposição 3.5 Existe uma família $\mathcal{G}$ de subconjuntos $G_{\delta}^{\prime}$ de ${ }_{2} 2$ que é bem-ordenada por $\subset$ com tipo de ordem $\omega_{1}$ e tal que $\bigcup \mathcal{G}={ }^{\omega_{2}}$.

Demonstração Mostraremos que vale o "resultado dual" para subconjuntos $F_{\sigma}$, i.e., que existe $\mathcal{F}$, família de subconjuntos $F_{\sigma}$ de ${ }^{\omega_{2}}$, bem ordenada por $\supset$ com tipo de ordem $\omega_{1}$ e tal que $\bigcap \mathcal{F}=\emptyset$. Dando a $\mathcal{P}(\omega)$ a topologia produto (identificando-o naturalmente com ${ }^{\omega} 2$, via funções característica ), acharemos tal $\mathcal{F}$ formada por subconjuntos $F_{\sigma}$ de $\mathcal{P}(\omega)$.

Seja $\left\langle\left\{A_{\xi}: \xi<\omega_{1}\right\},\left\{B_{\xi}: \xi<\omega_{1}\right\}\right\rangle$ um gap de Hausdorff em $\left\langle\mathcal{P}(\omega), C^{*}\right\rangle$. Considere, para cada $\xi<\omega_{1}$,

$$
J_{\xi}=\left\{C \in \mathcal{P}(\omega): A_{\xi} \subseteq^{*} C \subseteq^{*} B_{\xi}\right\}
$$

e, para $\xi<\omega_{1}$ e $n<\omega$ quaisquer, 


$$
J_{\xi, n}=\left\{C \in \mathcal{P}(\omega): A_{\xi} \backslash n \subseteq C \backslash n \subseteq B_{\xi} \backslash n\right\}
$$

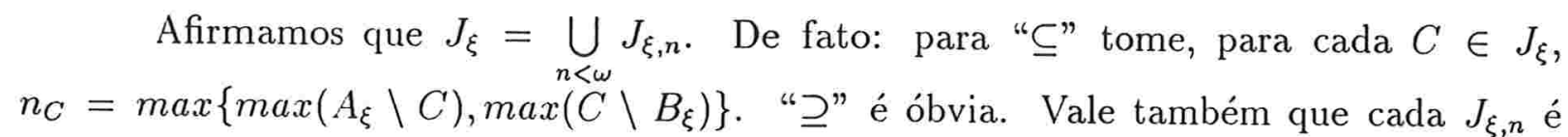
fechado (note que $\mathcal{P}(\omega) \backslash J_{\xi, n}=\left\{C \subseteq \omega: A_{\xi} \backslash n \nsubseteq C \backslash n\right\} \cup\left\{C \subseteq \omega: C \backslash n \nsubseteq B_{\xi} \backslash n\right\}$ e que cada um desses conjuntos que unimos é aberto), donde cada $J_{\xi}$ é um conjunto $F_{\sigma}$. É claro também que $\alpha<\beta<\omega_{1}$ implica $J_{\beta} \subset J_{\alpha}$, logo $\mathcal{F}=\left\{J_{\xi}: \xi<\omega_{1}\right\}$ está bem-ordenada por $\supset$ e enumerada pelo seu tipo de ordem. A hipótese de $\left\langle\left\{A_{\xi}: \xi<\omega_{1}\right\},\left\{B_{\xi}: \xi<\omega_{1}\right\}\right\rangle$ ser um gap implica $\bigcap \mathcal{F}=\emptyset$ e obtivemos uma família $\mathcal{F}$ nas condições desejadas.

\section{Notas}

A primeira seção deste capítulo adota a linha de exposição de fatos básicos a respeito de gaps que consta em [Sch93]. Já na segunda seção, a demonstração do Teorema 3.1 tenta explicitar os argumentos usados por Hausdorff em [Hau36] (que, além de estarem numa língua que não dominamos - o alemão -, não pareciam contar "todo o caminho das pedras"); nesse sentido, incluimos uma cuidadosa demonstração da não-existência de $\left\langle 1,1^{*}\right\rangle$-gaps em $\left\langle{ }^{\omega} \omega,\left\langle{ }^{*}\right\rangle\right.$, apenas para clarear as idéias. O Lema 3.1 (usado na prova de (i) $\Rightarrow($ ii $)$ da Proposição 3.2) é o Lema 10, p.122 de [Rot41]. A Proposição 3.3 é "folklore" e a Proposição 3.4 consta sem demonstração em [Sch93].

A demonstração do Teorema 3.4 está como na seção 4 de [vD84]. Modificamos bastante a parte final da demonstração do Teorema 3.5 ( "existência dos gaps de Hausdorff") com relação à que consta na seção 4 de [vD84], pois esta não nos pareceu muito cuidadosa. A Proposição 3.5 consta como exercício em [vD84], praticamente com mesmo enunciado (4.5, p.126), e aparece com o enunciado "dual" (com $F_{\sigma}$ 's ao invés de $G_{\delta}$ 's) em [JW97] (Afirmação 20.3, pág.118). 


\section{Capítulo 4}

\section{Algumas Aplicações à Topologia}

Neste capítulo, destacaremos algumas aplicações dos pequenos cardinais à Topologia. Determinadas "notas históricas e bibliográficas" estão inseridas no próprio texto o que, neste caso, nos pareceu melhor do que isolá-las no final do capítulo.

\subsection{Espaços seqüencialmente compactos, enumeravelmente compactos e correlatos}

\subsubsection{Peso, caráter e cardinalidade de (enumeravelmente) compactos não seqüencialmente compactos}

Os dois teoremas a seguir determinam limites inferiores (os melhores possíveis !) para o peso e para o caráter de espaços (enumeravelmente) compactos que não sejam seqüencialmente compactos usando pequenos cardinais.

Teorema $4.1 \quad \mathfrak{s}=\mathfrak{s}_{p}=\mathfrak{s}_{c}=\mathfrak{s}_{e c}$, onde

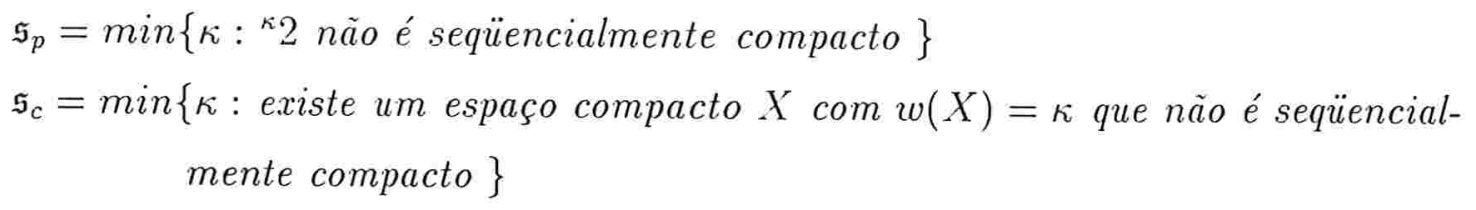



$\mathfrak{s}_{e c}=\min \{\kappa:$ existe um espaço enumeravelmente compacto $X$ com $w(X)=\kappa$ que não é seqüencialmente compacto $\}$

Demonstração Mostraremos que $\mathfrak{s} \leqslant \mathfrak{s}_{e c} \leqslant \mathfrak{s}_{c} \leqslant \mathfrak{s}_{p} \leqslant \mathfrak{s}$.

Prova de $\mathfrak{s}_{e c} \leqslant \mathfrak{s}_{c}$ : Espaços compactos são enumeravelmente compactos.

Prova de $\mathfrak{s}_{c} \leqslant \mathfrak{s}_{p}:{ }^{\kappa} 2$ é compacto e $w\left({ }^{\kappa} 2\right)=\kappa$ para todo cardinal infinito $\kappa$.

Prova de $\mathfrak{s} \leqslant \mathfrak{s}_{e c}$ : Seja $X$ um espaço enumeravelmente compacto com $w(X)<\mathfrak{s}$; mostraremos que $X$ é seqüencialmente compacto, donde $\mathfrak{s}_{e c}<\mathfrak{s}$ não pode ocorrer. De fato: seja $N \subseteq X$ enumerável e infinito e $\mathcal{B}$ uma base para $X$ com $|\mathcal{B}|<\mathfrak{s}$. Definindo $\mathcal{B}^{\prime}=\{B \in \mathcal{B}:|B \cap N|=\omega\}$, temos $\left|\mathcal{B}^{\prime}\right| N|\leqslant| \mathcal{B} \mid<\mathfrak{s}$ e, identificando $N$ com $\omega$, podemos afirmar que $\mathcal{B}^{\prime}\left\lceil N\right.$ não é splitting com relação a $[N]^{\omega}$, donde existe $A \subseteq N$ infinito e tal que

$$
{ }^{-}\left(\forall B \in \mathcal{B}^{\prime}\right)\left[\left(A \subseteq \subseteq^{*} B \cap N\right) \vee\left(A \subseteq^{*} N \backslash B\right)\right]
$$

Como $X$ é enumeravelmente compacto, $A$ possui um ponto de acumulação completo $x$. Afirmamos que $A$ converge para $x$. De fato, seja $U$ uma vizinhança qualquer de $x$; existe então $B \in \mathcal{B}$ tal que $x \in B \subseteq U$. Daí $B \in \mathcal{B}^{\prime}$ claramente e $|B \cap A|=\omega$, logo $A \nsubseteq \nsubseteq^{*} N \backslash B$ e tem-se $A \subseteq{ }^{*} B \cap N$. Assim, $B$ contém todos os pontos de $A$ a menos de no máximo um número finito deles; portanto, $A$ converge para $x$, conforme desejado.

Prova de $\mathfrak{s}_{p} \leqslant \mathfrak{s}$ : Bastará mostrar que, se $\mathcal{S} \subseteq[\omega]^{\omega}$ é uma família splitting, então $\mathcal{S}_{2}$ não é seqüencialmente compacto. $\mathrm{O}$ argumento é inteiramente análogo ao que fizemos na demonstração de $\omega_{1} \leqslant \mathfrak{s}$ : definimos uma seqüência $\left\langle f_{n}: n<\omega\right\rangle$ de elementos de $\mathcal{S}_{2}$ pondo, para cada $n<\omega$ e $S \in \mathcal{S}$,

$$
f_{n}(S)= \begin{cases}1 & \text { se } n \in S \\ 0 & \text { caso contrário }\end{cases}
$$

Afirmamos que $\left\langle f_{n}: n<\omega\right\rangle$ não pode possuir uma subseqüência convergente. De fato: suponha por absurdo que $\left\langle f_{n_{k}}: k<\omega\right\rangle$ seja uma subseqüência convergente, e considere $A=\left\{n_{k}: k<\omega\right\}$. Fixe $S \in \mathcal{S}$; temos então $\left\langle f_{n_{k}}\left(S^{\prime}\right): k<\omega\right\rangle$ convergente e portanto quase-constante. Se $f_{n_{k}}$ convergir para 1 , teremos $|A \backslash S|<\omega$, e se convergir 
para 0 , teremos $\left|A \cap S^{\prime}\right|<\omega$. Logo $\forall S \in \mathcal{S}[(|A \cap S|<\omega) \vee(|A \backslash S|<\omega)]$, o que contradiz a hipótese de $\mathcal{S}$ ser splitting.

Observamos que, se tomarmos $\mathcal{S}$ splitting com $|\mathcal{S}|=\mathfrak{c}$ - o que é fácil - e aplicarmos o argumento utilizado em $\mathfrak{s}_{p} \leqslant \mathfrak{s}$, podemos concluir diretamente que '2 não é seqüencialmente compacto; esse é essencialmente o exemplo 5.3 de [SS66]. Na verdade, como a propriedade "ser seqüencialmente compacto" é preservada por aplicações contínuas sobrejetoras ([Eng89], 3.10.32), s'2 não-seqüencialmente compacto implica que ${ }^{\kappa} 2$ não é seqüencialmente compacto, para todo $\kappa \geqslant \mathfrak{s}\left(\right.$ se $\kappa \geqslant \mathfrak{s}$, a "projeção" $\phi:{ }^{\kappa} 2 \mapsto{ }^{\mathfrak{s}} 2$ dada por $\phi(f)=f\lceil\mathfrak{s}$ é contínua, sobrejetora, aberta.).

Teorema $4.2 \quad \mathfrak{p}=\mathfrak{p}_{p}=\mathfrak{p}_{c}=\mathfrak{p}_{e c}=\mathfrak{p}_{\chi}$, onde

$$
\begin{aligned}
& \begin{aligned}
\mathfrak{p}_{p}= & \min \left\{\kappa:{ }^{\kappa} 2 \text { não é subseqüencial }\right\} \\
\mathfrak{p}_{c}= & \min \{\kappa: \text { existe um espaço compacto } X \text { com } \chi(X)=\kappa \text { que não é subseqüen- } \\
& \text { cial }\}
\end{aligned} \\
& \mathfrak{p}_{\text {ec }}=\min \{\kappa: \text { existe um espaço enumeravelmente compacto } X \text { com } \chi(X)=\kappa \text { que } \\
& \quad \text { não é subseqüencial }\} \\
& \mathfrak{p}_{X}=\min \{\kappa: \text { existe um espaço } X \text { com } \chi(X)=\kappa \text { que não é subseqüencial }\}
\end{aligned}
$$

Demonstração Mostraremos que $\mathfrak{p} \leqslant \mathfrak{p}_{\chi} \leqslant \mathfrak{p}_{e c} \leqslant \mathfrak{p}_{c} \leqslant \mathfrak{p}_{p} \leqslant \mathfrak{p}$. Observando que $\chi\left({ }^{\kappa} 2\right)=\kappa$ para todo cardinal infinito $\kappa$, as desigualdades $\mathfrak{p}_{\chi} \leqslant \mathfrak{p}_{e c} \leqslant \mathfrak{p}_{c} \leqslant \mathfrak{p}_{p}$ ficam inteiramente análogas às desigualdades $\mathfrak{s}_{e c} \leqslant \mathfrak{s}_{c} \leqslant \mathfrak{s}_{p}$ do teorema anterior.

Prova de $\mathfrak{p} \leqslant \mathfrak{p}_{\chi}$ : Seja $X$ um espaço com $\chi(X)<\mathfrak{p}$; mostraremos que $X$ é subseqüencial, donde $\mathfrak{p}_{\chi}<\mathfrak{p}$ não pode ocorrer. Seja então $N \subseteq X$ enumerável e infinito e $x$ um ponto de acumulação completo de $N$; de $\chi(x, X) \leqslant \chi(X)<\mathfrak{p}$ posso tomar $\mathcal{B}$ base local em $x$ com $|\mathcal{B}|<\mathfrak{p}$. Note que $\mathcal{B} \mid N$ possui a s.f.i.p. e, identificando $N \operatorname{com} \omega,|\mathcal{B}| N \mid<\mathfrak{p}$ nos dá um conjunto $A$ que é pseudo-intersecção infinita de $\mathcal{B}\lceil N$, e podemos supor s.p.g. que $A \subseteq N$; e é imediato agora que $A$ converge para $x$, pois $A$ está quase contido em qualquer vizinhança de $x$.

Prova de $\mathfrak{p}_{p} \leqslant \mathfrak{p}$ : Inicialmente observamos que a propriedade "ser subseqüencial" é hereditária, logo basta exibirmos um subespaço de ${ }^{\mathfrak{p} 2}$ que não seja subseqüencial e teremos 
estabelecida a desigualdade. Sabe-se que qualquer espaço zero-dimensional $X T_{1}$ com $w(X) \leqslant \kappa$ pode ser imerso em ${ }^{\kappa} 2$ ([Eng89],6.2.16), logo a questão pode ser limitada à procura de um espaço zero-dimensional $X, T_{1}$, com $w(X) \leqslant \mathfrak{p}$ e que não seja subseqüencial. Considere então $\mathcal{U} \subseteq[\omega]^{\omega} \operatorname{com}|\mathcal{U}|=\mathfrak{p}$ e tal que $\mathcal{U}$ satisfaça a s.f.i.p. e não possua pseudo-interseç̧ão infinita; é conveniente assumirmos que $\mathcal{U}$ é fechado para intersecções finitas e que $\bigcap \mathcal{U}=\emptyset$. Damos então a $X=\omega \cup\{\mathcal{U}\}$ a seguinte topologia: os pontos de $\omega$ são declarados isolados e as vizinhanças básicas de $\mathcal{U}$ são da forma $\{\mathcal{U}\} \cup U$ para $U \in \mathcal{U}$. Então $w(X) \leqslant|\mathcal{U}|+\omega=\mathfrak{p}$ e $X$ é zero-dimensional, pois $\bigcap \mathcal{U}=\emptyset$ implica que todos os naturais são fechados e $X \backslash(\{\mathcal{U}\} \cup U)=\omega \backslash U$ é aberto para todo $U \in \mathcal{U}$. Como acabamos de verificar que todos os pontos de $X$ são fechados, $X$ é $T_{1}$. E note ainda que $X$ não é subseqüencial, pois $\mathcal{U}$ é ponto de acumulação completo de $\omega$ mas não pode existir $A \subseteq \omega$ infinito que convirja para $\mathcal{U}$ pois um conjunto $A$ nessas condições seria pseudo-intersecção infinita de $\mathcal{U}$. Assim, $X$ é um espaço que pode ser imerso em ${ }^{\mathfrak{p} 2} 2$ e que não é subseqüencial, donde ${ }^{\mathfrak{p} 2}$ não é subseqüencial.

Trataremos agora da cardinalidade de espaços compactos Hausdorff que não sejam seqüencialmente compactos.

Teorema 4.3 Se $X$ é compacto $T_{2}$ e não é seqüencialmente compacto, então $2^{\mathfrak{t}} \leqslant|X|$.

Note que o enunciado do teorema implica que a afirmação "todo espaço compacto $T_{2}$ de cardinalidade $\leqslant \mathfrak{c}$ é seqüencialmente compacto" seja consistente: basta tomarmos um modelo em que $\mathfrak{t}=\mathfrak{c}([$ Vau84], Corol.5.10).

Demonstração Lembrando que, se $A \subseteq X, A^{\prime}$ é o derivado de $A$ (isto é, é o conjunto dos pontos de acumulação de $A$ ), observamos que, assumindo $T_{2}$,

(1) $(\forall A, B \in \mathcal{P}(X))\left[A \subseteq^{*} B \Rightarrow A^{\prime} \subseteq B^{\prime}\right]$

já que, para $X$ satisfazendo pelo menos $T_{1}$, um ponto de acumulação de $A$ possui em qualquer sua vizinhança infinitos pontos de $A$ (e, supondo $A \subseteq \subseteq^{*} B$, infinitos pontos de $B$ também). Vale ainda lembrar que, em espaços que sejam pelo menos $T_{1}$, os pontos de acumulação de subconjuntos enumeráveis infinitos são pontos de acumulação completos. 
Seja então $N \subseteq X$ enumerável e infinito e tal que nenhum de seus subconjuntos infinitos convirja. Como $X$ é compacto $T_{2}, X$ é regular e vale claramente que

(2) $\left(\forall T \in[N]^{\omega}\right)\left(\exists T_{0}, T_{1} \in[T]^{\omega}\right)\left[T_{0}^{\prime} \cap T_{1}^{\prime}=\emptyset\right]$

Mostraremos agora que para cada $\zeta<\mathfrak{t}$ existe uma família $\left\{T_{f}: f \in{ }^{\zeta} 2\right\} \subseteq[N]^{\omega}$ satisfazendo

(3) $\left(\forall f \in{ }^{\zeta} 2\right)(\forall \xi<\zeta)\left[T_{f} \subseteq^{*} T_{f \mid \xi}\right]$

(4) $\left(\forall f, g \in \varsigma^{\zeta}\right)\left[f \neq g \Rightarrow T_{f}^{\prime} \cap T_{g}^{\prime}=\emptyset\right]$

e a justificativa para a existência dessas famílias é análoga à demonstração do item $(c)$ do Teorema 2.1. Para $\zeta=0$, tomamos $T_{\emptyset}=N$. Para $0<\zeta<\mathfrak{t}$, supomos que as famílias $\left\{T_{f}: f \in{ }^{\xi} 2\right\}$ estejam bem construídas para todo $\xi<\zeta$ e dividimos a construção do caso $\zeta$ em dois casos. Se $\zeta=\delta+1$, tomamos para cada $f \in{ }^{\delta} 2$ subconjuntos infinitos $T_{f, 0}$ e $T_{f, 1}$ de $T_{f}$ satisfazendo $T_{f, 0}^{\prime} \cap T_{f, 1}^{\prime}=\emptyset$, usando (2), e para cada $g \in \varsigma_{2}$ definimos $T_{g}=T_{g \mid \delta, g(\delta)}$. Se $\zeta$ é ordinal limite, tomamos, para cada $f \in \varsigma_{2}, T_{f}$ uma pseudo-intersecção infinita de $\left\{T_{f \mid \xi}: \xi<\zeta\right\}$. (3) é óbvio para os dois casos. Para (4), sejam $f, g$ distintas em $\varsigma_{2}$ e considere $\beta=\min \{\xi<\zeta: f(\xi) \neq g(\xi)\}$. Se $\beta+1=\zeta$, a construção feita para o caso

" $\zeta$ sucessor " garante (4). Se $\beta+1<\zeta, T_{f \mid \beta+1}^{\prime} \cap T_{g \mid \beta+1}^{\prime}=\emptyset$ por hipótese de indução e (1) e (3) nos dão $T_{f}^{\prime} \cap T_{g}^{\prime} \subseteq T_{f \mid \beta+1}^{\prime} \cap T_{g \mid \beta+1}^{\prime}$. Portanto, a existência dessas famílias está justificada.

Note agora que, sendo $X$ compacto, $T^{\prime}$ é um fechado não-vazio sempre que $T \subseteq X$ é infinito. Segue que, para cada $f \in{ }^{\mathrm{t}} \cdot 2, \bigcap_{\zeta<\mathrm{t}} T_{f \backslash \zeta}^{\prime}$ é não vazio, já que (3), (1) e a observação do início deste parágrafo garantem que, para toda $f \in{ }^{\mathfrak{t}} 2,\left\{T_{f \mid \zeta}^{\prime}: \zeta<\mathfrak{t}\right\}$ é uma família de fechados com a p.i.f. no compacto $X$. Definimos então uma aplicação $S$ : ' $2 \mapsto \mathcal{P}(X) \backslash\{\emptyset\}$ pondo $S(f)=\bigcap_{\zeta<\mathrm{t}} T_{f \mid \zeta}^{\prime}$. (1), (3) e (4) nos dão agora $\left(\forall f, g \in^{\mathrm{t}} 2\right)[f \neq g \Rightarrow S(f) \cap S(g)=\emptyset]$. Daí, tomando $x_{f} \in S(f)$ para cada $f \in \mathfrak{t}^{\mathfrak{2}} 2$, obtemos $\left\{x_{f}: f \in \mathfrak{t}^{\mathfrak{t}}\right\}$ que é um subconjunto de $X$ com cardinalidade $2^{\mathrm{t}}$ e mostramos o desejado.

Corolário Suponha $2^{t}>\mathfrak{c}$. Então as seguintes afirmações sobre um espaço compacto $T_{2}$ $X$ são equivalentes:

(a) Todo subespaço enumeravelmente compacto de $X$ é fechado. 
(b) $X$ é sequencial, isto é, para cada $A \subseteq X$, se $A$ não for fechado, então algum subconjunto enumerável e infinito de $A$ converge para um ponto de $X \backslash A$.

Demonstração (do Corolário) $(b) \Rightarrow(a)$ : Imediato, mesmo que o espaço seqüencial em questão não seja compacto (e sem usar " $2^{\mathrm{t}}>\mathfrak{c}$ "): supondo por absurdo que $Y \subseteq X$ seja enumeravelmente compacto e não-fechado, (b) nos fornece um subconjunto enumerável infinito $Z$ de $Y$ que converge para um ponto de $X \backslash Y$, o que é uma contradição. Logo, qualquer espaço seqüencial é tal que seus subespaços enumeravelmente compactos são fechados.

$(a) \Rightarrow(b)$ : Seja $A \subseteq X$ um subconjunto não-fechado. Então, $A$ não é enumeravelmente compacto, e portanto existe $N \subseteq A$ infinito, enumerável, fechado e discreto. Afirmamos que algum subconjunto infinito de $N$ converge (necessariamente para um ponto de $X \backslash A$ ). De fato: suponhamos por absurdo que nenhum subconjunto infinito de $N$ convirja. Então o compacto $\bar{N}^{X}$ de $X$ não é sequencialmente compacto, daí $\left|\bar{N}^{X}\right| \geqslant 2^{\mathrm{t}}>$ c. Porém, isso é um absurdo pois o fato a seguir (que se baseia numa construção de tipo bastante conhecido) nos dará $\left|\bar{N}^{X}\right| \leqslant \mathfrak{c}$, já que será garantida a existência de um $Y \subseteq X$ enumeravelmente compacto com $|Y| \leqslant c$ e $N \subseteq Y \subseteq \bar{N}^{X}$; mas, temos $Y$ subespaço enumeravelmente compacto de $X$ e portanto fechado, por $(a)$, logo $Y=\bar{N}^{X}$.

Fato 4.1 Se $Z$ é enumeravelmente compacto, então para cada $S \subseteq Z$ infinito existe um $T$ enumeravelmente compacto satisfazendo $S \subseteq T(\subseteq \bar{S})$ e $|T| \leqslant|S|^{\omega}$.

Prova do Fato: Sejam $Z$ e $S$ como no enunciado. Para cada $N \in[Z]^{\omega}$ escolhemos $p(N)$ um ponto de acumulação completo de $N$. Agora, por recursão em $\zeta<\omega_{1}$, definimos uma família crescente $\left\{S_{\zeta}: \zeta<\omega_{1}\right\}$ de subconjuntos de $Z$ satisfazendo $S_{0}=S$ e

$$
S_{\zeta}=\bigcup_{\xi<\zeta} S_{\xi} \cup\left\{p(N): N \in\left[\bigcup_{\xi<\zeta} S_{\xi}\right]^{\omega}\right\} \text { para } 0<\zeta<\omega_{1}
$$

e pomos $T=\bigcup_{\zeta<\omega_{1}} S_{\zeta}$. Por indução pode-se provar facilmente que $S \subseteq S_{\zeta} \subseteq \bar{S}$ e $\left|S_{\zeta}\right| \leqslant|S|^{\omega}$ para todo $\zeta<\omega_{1}$, daí $S \subseteq T \subseteq \bar{S}$ e $\omega_{1} \leqslant|S|^{\omega}$ dá $|T| \leqslant|S|^{\omega}$. A regularidade de $\omega_{1}$ faz com que qualquer subconjunto infinito e enumerável $I$ de $T$ esteja contido num dos $S_{\zeta}$, e assim 
o ponto de acumulação completo de $I$ escolhido inicialmente satisfaz $p(I) \in S_{\zeta+1} \subseteq T$ e portanto $T$ é enumeravelmente compacto.

O Teorema 4.3 e o seu corolário motivaram as seguintes perguntas de van Douwen (a exemplo de Vaughan em seu artigo [Vau90] no Open Problems, respeitaremos a numeração original do artigo [vD84] quando nos referirmos a questões apresentadas por van Douwen em seu artigo no Handbook, mesmo quando as enunciarmos de modo um pouco diferente.).

Questão 6.6 (van Douwen) A limitação inferior apresentada no Teorema 4.3 é a melhor possivel ? Isto é, existe um espaço compacto $T_{2}$ de cardinalidade $2^{\mathrm{t}}$ que não seja seqüencialmente compacto?

Questão 6.7 (van Douwen) $O$ corolário do Teorema 4.3, enunciado com a hipótese adicional "c $<2^{\mathrm{t}}$ ", vale em $Z F C$ ? Ou, como apenas a implicação $(a) \Rightarrow(b)$ usou a hipótese adicional, a pergunta pode ser colocada da seguinte forma: em ZFC, "todo subespaço enumeravelmente compacto de $X$ (compacto, $T_{2}$ ) é fechado" implica "X é seqüencial"?

Em seu artigo no Open Problems ([Vau90], Vaughan fez um breve relato da situação em que se encontravam aqueles problemas. 6.7 estava em aberto, e continua ainda nessa situação, segundo o acompanhamento periódico sobre o status dos problemas do Open Problems que é publicado na revista Topology and its Applications. Já para a questão 6.6, Alan Dow construiu um modelo em que todos os espaços compactos $T_{2}$ de cardinalidade $2^{t}$ são seqüencialmente compactos. A solução de Alan Dow usou fortemente o cardinal $\mathfrak{n}$, definido como o "número de Novák" de $\beta \omega \backslash \omega$, que é a menor cardinalidade de uma cobertura de $\beta \omega \backslash \omega$ por conjuntos raros ("nowhere dense"). Dow observou que, se $X$ é compacto $T_{2}$ e não seqüencialmente compacto, então $\mathfrak{n} \leqslant|X|$, mas no modelo que ele construiu valia a desigualdade estrita $2^{\mathfrak{t}}<\mathfrak{n}$.

Para espaços enumeravelmente compactos $T_{3}$, porém, temos um limite inferior que é o melhor possível para a cardinalidade dos não-seqüencialmente compactos.

Teorema 4.4 $\mathfrak{c}=\min \{|X|: X$ é um espaço enumeravelmente compacto e regular que não é seqüencialmente compacto $\}$

Demonstração Seja $\lambda$ o cardinal definido pela expressão à direita: os primeiros $\omega$ passos 
da recursão feita da demonstração do Teo. 4.3 nos dão $c \leqslant \lambda$ (lembrando que, em enumeravelmente compactos, famílias enumeráveis de fechados com a p.i.f. têm intersecção não vazia). Para $\lambda \leqslant \mathfrak{c}$, tomamos um espaço compacto $T_{2} Z$ não-seqüencialmente compacto (por exemplo, ${ }^{5} 2$ ou $\beta \omega$ ) e tomamos $S \subseteq Z$ infinito enumerável e tal que nenhum de seus subconjuntos infinitos convirja; o espaço $T \supseteq S$ dado pelo Fato 4.1 nos dá a desigualdade desejada.

\subsubsection{Produtos. Problemas do tipo de Scarborough e Stone}

Em 1966, o clássico artigo [SS66] de Scarborough e Stone deu importantes contribuições para o estudo da seguinte questão: se $P$ é uma propriedade similar porém mais fraca do que a compacidade, $P$ é preservada por produtos ? Ou seja, a pergunta é "vale o análogo do Teorema de Tychonoff para a propriedade $P$ ?" ou "a propriedade $P$ é produtiva ?". Já se sabia que nem sempre poderiam ser estabelecidas essas preservações para produtos, pois Novák e Teresaka no início dos anos 50 exibiram espaços enumeravelmente compactos $X$ e $Y$ tais que $X \times Y$ não é enumeravelmente compacto (ver [Vau84],3.1.,p.576). Para seqüencialmente compactos, porém, alguma preservação foi garantida. O teorema a seguir é uma extensão ao cardinal $\mathfrak{t}$, devida a Hechler ([Hec75b]), de um argumento original de Scarborough e Stone que usava o cardinal $\omega_{1}$. Porém, o próprio Hechler chamou a atenção para o fato de que as demonstrações originais (de Scarborough e Stone) apenas exigiam que se conseguisse uma pseudo-interseção infinita para famílias bem-ordenadas por $\supseteq$ de conjuntos infinitos e enumeráveis: assim, a condição "menos do que $\omega_{1}$ espaços" pôde ser substituída por "menos do que $\mathfrak{t}$ espaços".

Teorema 4.5 (a) Qualquer produto de menos do que $t$ espaços seqüencialmente compactos é seqüencialmente compacto.

(b) Qualquer produto de no máximo $t$ espaços seqüencialmente compactos é enumeravelmente compacto.

Demonstração Seja $\kappa \leqslant \mathfrak{t}$ e $\left\{X_{\zeta}: \zeta<\kappa\right\}$ uma família de espaços seqüencialmente compactos; denotaremos por $\prod_{\eta}: \prod_{\zeta<\kappa} X_{\zeta} \mapsto X_{\eta}$ a projeção do produto na $\eta$-ésima coordenada. Seja $N$ um subconjunto enumerável e infinito qualquer de $\prod_{\zeta<\kappa} X_{\zeta}$. 
Por recursão transfinita em $\zeta<\kappa$, construiremos $T_{\zeta} \in[N]^{\omega}$ e $x_{\zeta} \in X_{\zeta}$ para todo $\zeta<\kappa$ satisfazendo

(1) $(\forall \xi<\zeta)\left[T_{\zeta} \subseteq^{*} T_{\xi}\right]$

(2) $\prod_{\zeta}\left[T_{\zeta}\right]=\left\{x_{\zeta}\right\}$ ou $\prod_{\zeta}\left[T_{\zeta}\right]$ é infinito e converge para $x_{\zeta}$.

Afirmamos ainda que tal construção é tal que, se $\kappa<\mathfrak{t}$, então existe $T \in[N]^{\omega}$ satisfazendo

(3) $(\forall \zeta<\kappa)\left[T \subseteq \subseteq^{*} T_{\zeta}\right]$

A justificativa da construção é como segue:

$\zeta=0$. Se $\prod_{0}[N]$ for finito, existem $x_{0} \in \prod_{0}[N]$ e $T_{0} \in[N]^{\omega}$ tais que $\prod_{0}\left[T_{0}\right]=$ $\left\{x_{0}\right\}$. Caso contrário, como $X_{0}$ é seqüencialmente compacto, existe $A \subseteq \prod_{0}[N]$ infinito que converge para um determinado $x_{0} \in X_{0}$; tomamos então $T_{0}=\prod_{0}^{-1}[A] \cap N$.

Para $0<\zeta<\kappa$, supomos $\left\{T_{\xi}: \xi<\zeta\right\}$ bem construída e dividimos a construção de $T_{\zeta}$ em dois casos:

$\zeta$ ordinal sucessor: Suponha $\zeta=\delta+1$ e considere o conjunto $\prod_{\zeta}\left[T_{\delta}\right]$ de $X_{\zeta}$; argumentos análogos aos usados no caso " $\zeta=0$ " nos dão $T_{\zeta} \subseteq T_{\delta}$ infinito e $x_{\zeta} \in X_{\zeta}$ satisfazendo uma das condições de (2). (1) está obviamente satisfeita.

$\zeta$ ordinal limite: Tome $T_{\zeta}^{\prime}$ uma pseudo-interseç̧ão infinita de $\left\{T_{\xi}: \xi<\zeta\right\}$ e trabalhe com $\prod_{\zeta}\left[T_{\zeta}^{\prime}\right]$ obtendo $T_{\zeta} \subseteq T_{\zeta}^{\prime}$ e $x_{\zeta} \in X_{\zeta}$ numa das condições de (2). (1) está obviamente satisfeita.

Para verificar (3) no caso de $\kappa<\mathfrak{t}$, basta ver que $\kappa<\mathfrak{t}$ implica que $\left\{T_{\zeta}: \zeta<\kappa\right\}$ possui uma pseudo-intersecção infinita $T$. Assim, a construção está bem definida e satisfaz as propriedades desejadas.

Mostraremos agora que $x=\left\langle x_{\zeta}: \zeta<\kappa\right\rangle$ é um ponto de acumulação completo de $N$ (o que prova o item $(b)$ ) e que, se $\kappa<\mathfrak{t}$, então $T$ é um subconjunto infinito de $N$ que converge para $x$ (o que prova o item $(a)$ ). De fato: considere $B$ um aberto básico de $\prod_{\zeta<\kappa} X_{\zeta}$ que contenha $x$; então $B$ é da forma $\bigcap_{\zeta \in F} \prod_{\zeta}^{-1}\left[B_{\zeta}\right]$, onde $\emptyset \neq F \subset \kappa$ é finito e $B_{\zeta}$ é uma vizinhança aberta de $x_{\zeta}$ para $\zeta \in F$. Temos agora que, para cada $\zeta \in F$, todos a menos de máximo finitos elementos de $T_{\zeta}$ pertencem a $\prod_{\zeta}^{-1}\left[B_{\zeta}\right]$, por (2); já (1) nos dá que $T_{\max (F)} \subseteq^{*} T_{\zeta}$ para todo $\zeta \in F$ e portanto todos a menos de no máximo finitos elementos 
de $T_{\max (F)}$ pertencem a $B$, donde $|B \cap N|=\omega$ e $x$ é ponto de acumulação completo de $N$; e se $\kappa<\mathfrak{t}$ teremos $T \subseteq \subseteq^{*} T_{\max (G)}$ para qualquer que seja $\emptyset \neq G \subset \kappa$ finito, logo $T$ converge para $x$.

Temos agora uma questão interessante: sabemos que ${ }^{\mathfrak{2} 2}$ não é seqüencialmente compacto, pelo Teo. 4.1. Porém, qualquer produto de menos do que $\mathfrak{t}$ seqüencialmente compactos é seqüencialmente compacto. Então $t \leqslant \mu \leqslant \mathfrak{s}$, onde

$$
\begin{gathered}
\mu=\min \{\kappa: \text { algum produto de } \kappa \text { espaços seqüencialmente compactos não é } \\
\text { seqüencialmente compacto }\}
\end{gathered}
$$

Questão 6.10 (van Douwen) $\mu$ é um cardinal conjuntístico, isto é, $\mu$ pode ser definido a partir de condições extraídas tão-somente da teoria dos conjuntos?

Essa pergunta teve resposta ainda nos anos 80: Nyikos, Pelant e Simon, e Fric e Vojtás (independentemente) mostraram que $\mu=\mathfrak{h}$, onde $\mathfrak{h}$ é um pequeno cardinal definido da seguinte forma: $\mathcal{D} \subseteq[\omega]^{\omega}$ é dita uma família densa se para cada $X \in[\omega]^{\omega}$ existe $Y \in \mathcal{D}$ tal que $Y \subset^{*} X$, e $\mathcal{D}$ é dita uma família aberta se, para quaisquer $X \in[\omega]^{\omega}$ e $Y \in \mathcal{D}$, $X \subset^{*} Y \Rightarrow X \in \mathcal{D}$. Dizemos que $\left\langle[\omega]^{\omega}, \subset^{*}\right\rangle$ é $\kappa$-distributivo se todo conjunto formado por menos que $\kappa$ famílias abertas densas tem intersecção não-vazia. Então h é o "número de distributividade",

$\mathfrak{h}=\min \left\{|\mathcal{F}|: \mathcal{F}\right.$ é um conjunto de famílias abertas densas em $\left.[\omega]^{\omega} \operatorname{com} \bigcap \mathcal{F}=\emptyset\right\}$

isto é, h é o menor cardinal $\lambda$ para o qual $\left\langle[\omega]^{\omega}, C^{*}\right\rangle$ não é $\lambda^{+}$-distributivo. O motivo para a escolha da letra $\mathfrak{h}$, porém, é topológico e é conseqüência de um resultado notável: pode-se provar em $Z F C$ que existem $\pi$-bases para $\beta \omega \backslash \omega$ que formam árvores quando ordenadas por $\supset$ e que

$\mathfrak{h}=\min \{\kappa$ : existe uma $\pi$-base para $\beta \omega \backslash \omega$ que forma uma árvore de altura ("height") $\kappa\}$

Referências bibliográficas sobre $\mathfrak{h}$ podem ser encontradas no artigo de Vaughan no Open Problems ([Vau90]).

Enunciar uma questão análoga à pergunta 6.10 de van Douwen, relacionando o 
produto de seqüencialmente compactos com a compacidade enumerável, torna-se, porém, um pouco mais complicado. De fato, resultados de consistência e necessidade de hipóteses sobre axiomas de separação (o que não ocorreu até este momento em nossa discussão sobre produtos) vêm à baila na discussão da seguinte questão.

\section{Questão 6.11 (van Douwen, reformulada por Vaughan) Qualquer produto de es- paços seqüencialmente compactos é enumeravelmente compacto?}

Este problema é freqüentemente chamado de "O Problema de Scarborough e Stone". Como as propriedades "ser enumeravelmente compacto" e "ser seqüencialmente compacto" são equivalentes para espaços seqüenciais, um "caso especial" desse problema poderia ser colocado da seguinte forma: A compacidade enumerável é produtiva para espaços de Hausdorff que satisfazem o primeiro axioma da enumerabilidade? (Problema 285 do Open Problems, [Nyi90]). Na época em que o artigo de van Douwen no Handbook foi escrito, não se conhecia em $Z F C$ um produto de seqüencialmente compactos que não fosse enumeravelmente compacto; sabia-se porém que em alguns modelos nos quais vale a igualdade "b $=\mathfrak{c} "$ existiam exemplos desse tipo (o exemplo 13.1,pg.159 de [vD84] mostra que "b $=\mathfrak{c} "$ é suficiente para que um tal produto exista.). Em 1987, Nyikos e Vaughan ([NV87]) conseguiram uma solução negativa para o problema em modelos nos quais vale $\mathfrak{b}<\mathfrak{c}$, e em 1992 ([NV92] esses mesmos autores apresentaram soluções distintas e negativas (em $Z F C$ ) para a classe dos espaços seqüencialmente compactos $T_{2}$ (mais comentários sobre os trabalhos de Nyikos e Vaughan na última subseção desta seção); porém, para espaços $T_{3}, T_{3 \frac{1}{2}}$ e $T_{4}$, a questão continua em aberto. Destacamos alguns comentários que constam em [NV92]: para a classe dos espaços $T_{6}$ e $T_{5}$ (respectivamente, espaços Hausdorff perfeitamente normais e hereditariamente normais) a solução do problema é independente de $Z F C$; existem ainda algumas classes de espaços nos quais uma solução apresentaria interesse, tais como espaços zero-dimensionais $T_{2}$, ou espaços zero-dimensionais $T_{2}$ localmente compactos, que são os tipos de espaços para os quais foram apresentadas soluções consistentes negativas, ou ainda espaços $T_{2}$ que satisfaçam o primeiro axioma da enumerabilidade. Os espaços $T_{2}$ com os quais Nyikos e Vaughan trabalharam em [NV92] não eram regulares, e portanto não eram nem zero-dimensionais e nem primeiro enumeráveis, pois espaços zero-dimensionais Hausdorff são completamente regulares e espaços de Hausdorff 
enumeravelmente compactos e que satisfazem o primeiro axioma da enumerabilidade são regulares. O problema de Scarborough e Stone também está aberto para a classe dos grupos topológicos.

\subsubsection{Espaços de $\subset^{*}$-boas-ordens e espaços de gaps}

Nesta subseção, apresentaremos dois exemplos de espaços construídos a partir de subfamílias de $[\omega]^{\omega}$ bem ordenadas por $C^{*}$. Esses exemplos serão utilizados durante todo o restante desta seção. O primeiro exemplo, a seguir, nos sugere uma maneira natural de se obter um espaço topológico a partir de uma $\subset^{*}$-boa ordem, "traduzindo-se" as propriedades conjuntísticas da $C^{*}$-boa ordem em propriedades topológicas do espaço. Tal espaço será ainda nosso primeiro exemplo do que denominaremos de $F R$-espaço (espaço de Franklin-Rajagopalan), por definição um espaço $T_{2}$ localmente compacto que possui um subconjunto discreto denso e enumerável cujo complementar é homeomorfo a um ordinal com a topologia da ordem.

Exemplo 4.1 Existe um espaço topológico não-compacto, separável, seqüencialmente compacto, localmente compacto e normal.

Prova: Usando a demonstração da igualdade $\mathfrak{t}=\mathfrak{t}_{2}$ no Teo. 2.4, podemos afirmar que existe $\mathcal{T} \subseteq[\omega]^{\omega}$ bem-ordenada por $\subset^{*}$ com tipo de ordem $t$ e que satisfaz

(1) $\left(\forall X \in[\omega]^{\omega}\right)(\exists T \in \mathcal{T})[|X \cap T|=\omega]$

A família $\mathcal{T}$ é obtida a partir dos complementares dos elementos de uma torre de tamanho mínimo.

Seja $Y=\omega \cup \mathcal{T}$. Damos a $Y$ a seguinte topologia: os pontos de $\omega$ são declarados isolados e as vizinhanças básicas de um elemento $T \in \mathcal{T}$ são da forma

$$
B(S, T, F)=\left\{A \in \mathcal{T}: S \subset^{*} A \subseteq^{*} T\right\} \cup((T \backslash S) \backslash F)
$$

onde $S \in \mathcal{T} \cup\{\emptyset\}$ satisfaz $S \subset^{*} T$ e $F \in[\omega]^{<\omega}$. Esses subconjuntos finitos de $\omega$ que aparecem nas expressões dos abertos básicos garantem que, se $T^{\prime} \in \mathcal{T}$ pertence à intersecção de dois abertos básicos $B\left(S_{1}, T_{1}, F_{1}\right)$ e $B\left(S_{2}, T_{2}, F_{2}\right)$ tais que $S_{1} \subseteq^{*} S_{2}$, então, 
fazendo $F^{\prime}=\left(T^{\prime} \backslash S_{2}\right) \backslash\left(\left(\left(T_{1} \backslash S_{1}\right) \backslash F_{1}\right) \cap\left(\left(T_{2} \backslash S_{2}\right) \backslash F_{2}\right)\right)$, tem-se $B\left(S_{2}, T^{\prime}, F^{\prime}\right) \subseteq$ $B\left(S_{1}, T_{1}, F_{1}\right) \cap B\left(S_{2}, T_{2}, F_{2}\right)$, portanto a topologia está bem definida.

$\mathcal{T}$ é um subespaço fechado de $Y$ que não é compacto, já que $\mathcal{T}$ é claramente homeomorfo ao cardinal $\mathfrak{t}$ com a topologia da ordem. Segue que $Y$ não é compacto.

$\omega$ é denso em $Y$ (note que, se $S$ e $T$ estão nas condições da definição dos abertos básicos, $T \backslash S$ é sempre infinito!), logo $Y$ é separável.

Verifiquemos agora que $Y$ é seqüencialmente compacto. Seja $N \subseteq Y$ infinito e enumerável; se $N \cap \mathcal{T}$ é infinito, podemos usar que $t$ é seqüencialmente compacto (já que $\mathfrak{t}=c f(\mathfrak{t})>\omega$, ver Exemplo 1.1) e que $\mathcal{T}$ e $\mathfrak{t}$ são homeomorfos e encontramos $N^{\prime} \subseteq$ $N \cap \mathcal{T} \subseteq N$ infinito e que converge para um elemento de $\mathcal{T}$. Se $N \cap \mathcal{T}$ é finito, $N \cap \omega$ é infinito e basta mostrar que qualquer subconjunto infinito $\omega$ possui um subconjunto infinito que converge (obviamente para um elemento de $\mathcal{T}$ também, pois os demais pontos são isolados.).

De fato: seja $I \in[\omega]^{\omega}$. Usando (1), podemos definir

$$
\begin{aligned}
M & =\subset^{*}-\min \{T \in \mathcal{T}:|I \cap T|=\omega\} \\
& =\subset^{*}-\min \{T \in \mathcal{T}:|B(\emptyset, T, \emptyset) \cap I|=\omega\}
\end{aligned}
$$

Afirmamos que $D=I \cap M=I \cap B(\emptyset, M, \emptyset)$ converge para $M$. Tomemos então uma vizinhança básica $B(S, M, F)$ de $M, \operatorname{com} \mathcal{T} \cup\{\emptyset\} \ni S \subset^{*} M$ e $F \in[\omega]^{<\omega}$. Temos

$$
\begin{aligned}
B(S, M, F) & =\left\{A \in \mathcal{T}: S \subset^{*} A \subseteq^{*} M\right\} \cup((M \backslash S) \backslash F) \\
& =\left(\left\{A \in \mathcal{T}: A \subseteq^{*} M\right\} \cup M\right) \backslash\left(\left\{A \in \mathcal{T}: A \subseteq^{*} S\right\} \cup S \cup F\right) \\
& =B(\emptyset, M, \emptyset) \backslash(B(\emptyset, S, \emptyset) \cup F)
\end{aligned}
$$

donde

$$
\begin{aligned}
D \backslash B(S, M, F) & =D \backslash(B(\emptyset, M, \emptyset) \backslash(B(\emptyset, S, \emptyset) \cup F)) \\
& =D \cap(B(\emptyset, S, \emptyset) \cup F) \\
& \subseteq I \cap(B(\emptyset, S, \emptyset) \cup F) \\
& ={ }^{*} I \cap B(\emptyset, S, \emptyset)
\end{aligned}
$$

que é finito pela $\subset^{*}$-minimalidade de $M$. 
$\therefore D$ converge para $M$, pois $S$ e $F$ são arbitrários, logo $Y$ é seqüencialmente compacto.

Note agora que, para $T \in \mathcal{T}$ qualquer, $B(\emptyset, T, \emptyset)$ é um subconjunto fechado de $Y$ (basta ver que, se $T \subset^{*} A \in \mathcal{T}$, então $B(T, A, \emptyset) \subseteq Y \backslash B(\emptyset, T, \emptyset)$ ), logo a expressão deduzida na verificação anterior, pondo $M=T$, nos dá que os abertos básicos $B(S, T, F)$ são fechados (já que é possível escrever $B(S, T, F)=B(\emptyset, T, \emptyset) \backslash(B(\emptyset, S, \emptyset) \cup F)$ ). Para todo $n<\omega,\{n\}$ também é fechado ("tomamos sempre $F=\{n\}$ "), logo todos os abertos básicos que demos são clopens e $Y$ é zero-dimensional. As "contas" que fizemos acima também nos dão que $Y$ é $T_{2}$, portanto $Y$ é completamente regular.

Para verificar que $Y$ é localmente compacto, mostraremos que todos os abertos básicos de $Y$ são compactos, o que é óbvio para os abertos da forma $\{n\}$ para todo $n<\omega$. Sejam então $T \in \mathcal{T}, S \in \mathcal{T} \cup\{\emptyset\} \operatorname{com} S \subset^{*} T, F \in[\omega]^{<\omega}$ e $\mathcal{U}$ uma cobertura aberta de $B(S, T, F)$ por abertos de $Y$. Existe $U_{0} \in \mathcal{U}$ tal que $T \in U_{0}$, logo existem $S_{1}$ e $F_{0}$ satisfazendo $S \subseteq \subseteq^{*} S_{1} \subset^{*} T=S_{0}, F_{0} \in[\omega]^{<\omega}$ e $B\left(S_{1}, S_{0}, F_{0}\right) \subseteq U_{0}$. Se $S \subset^{*} S_{1}$, o argumento pode ser repetido, tomando-se $U_{1} \in \mathcal{U}$ tal que $S_{1} \in U_{1}$, etc., obtendose $S \subseteq^{*} S_{2} \subset^{*} S_{1}$, etc. Mas, boas ordens não podem possuir seqüências decrescentes infinitas, e $\subset^{*}$ bem-ordena $\mathcal{T} \cup\{\emptyset\}$, logo existem $0<n<\omega, S_{i} \in \mathcal{T} \cup\{\emptyset\}$ para $i \leqslant n$, $F_{i} \in[\omega]^{<\omega}$ e $U_{i} \in \mathcal{U}$ para $i<n$ tais que $S_{0}=T, S_{n}=S$ e $B\left(S_{i+1}, S_{i}, F_{i}\right) \subseteq U_{i}$ para todo $i<n$. É claro agora que a família finita de abertos $\left\{U_{i}: i<n\right\} \subseteq \mathcal{U}$ cobre $B(S, T, F) \cap \mathcal{T}$. De

$$
\begin{aligned}
B(S, T, F) \cap \omega & =(T \backslash S) \backslash F \\
& =\left(\bigcup_{i<n}\left(S_{i} \backslash S_{i+1}\right)\right) \backslash F \\
& \subseteq\left(\left(\bigcup_{i<n} B\left(S_{i+1}, S_{i}, F_{i}\right)\right) \backslash F\right) \cup \bigcup_{i<n} F_{i} \\
& \subseteq \bigcup_{i<n} U_{i} \cup \bigcup_{i<n} F_{i}
\end{aligned}
$$

e de $\bigcup_{i<n} F_{i}$ ser finito conclui-se que $\mathcal{U}$ possui uma subcobertura finita.

$\therefore B(S, T, F)$ é compacto para $S, T$ e $F$ arbitrários, portanto $Y$ é localmente compacto.

Resta mostrar que $Y$ é normal. Inicialmente observamos que, dados dois fechados disjuntos quaisquer do subespaço $\mathcal{T}$ de $Y$, pelo menos um deles é compacto. De fato: como $\mathcal{T}$ e $\mathfrak{t}$ são homeomorfos, basta lembrar que $c f(\mathfrak{t})=\mathfrak{t}>\omega$ e portanto a intersecção 
de qualquer quantidade menor que $\mathfrak{t}$ de c.u.b.'s em $\mathfrak{t}$ é c.u.b. em $\mathfrak{t}$ (conforme destacamos no Cap.1 ), logo é impossível a existência de dois fechados disjuntos e cofinais em $\mathfrak{t}$; pelo menos um deles deve ser limitado e portanto compacto.

Com isso, podemos afirmar mais: dados dois fechados disjuntos quaisquer $F, G \subseteq$ $Y$, pelo menos um deles é compacto (e isso já garante que $Y$ é normal, pois já sabemos que $Y$ é completamente regular, em particular regular; o resto é rotina). De fato: sejam $F, G \subseteq Y$ fechados disjuntos; podemos supor s.p.g. que $F \cap \mathcal{T}$ é compacto. Seja então $\mathcal{U}$ uma cobertura aberta qualquer de $F$ por abertos de $Y$; existe então $\mathcal{V} \subseteq \mathcal{U}$ finito e tal que $F \cap \mathcal{T} \subseteq \cup \mathcal{V}$. Segue que $F \backslash \cup \mathcal{V} \subseteq \omega$, isto é, $F \backslash \bigcup \mathcal{V}$ é um subconjunto fechado e discreto do espaço enumeravelmente compacto $Y$; assim, $F \backslash \bigcup \mathcal{V}$ é finito e segue que $\mathcal{U}$ possui uma subcobertura finita.

$\therefore F$ é compacto e, como $F$ e $G$ são fechados disjuntos arbitrários e $Y$ é regular, $Y$ é normal.

No exemplo dado, o fato "de cada dois fechados disjuntos de $Y$, pelo menos um deles é compacto" é por si só suficiente para mostrar-se que o espaço em questão é localmente compacto, pois tal fato implica, em particular, que vale a condição "de cada dois fechados completamente separados de $Y$, pelo menos um deles é compacto", que é equivalente, usando que $Y$ é completamente regular, à condição " $Y$ é compacto ou $|\beta Y \backslash Y|=1 "$ ([Eng89], 3.12.16). Assim, temos $\beta Y \backslash Y$ unitário para o espaço $Y$ do exemplo e portanto $Y$ é homeomorfo a um subespaço aberto de um espaço compacto $T_{2}$, sendo portanto localmente compacto. No exemplo, justificamos a compacidade local de $Y$ via argumentos usando cobertura de abertos básicos porque argumentos inteiramente análogos serão necessários muito brevemente.

Ainda sobre o exemplo que acabamos de dar, observamos que, em modelos nos quais vale $\mathfrak{t}=\omega_{1}$, o espaço $Y$ construído satisfaz ainda o primeiro axioma da enumerabilidade. $Y$ é também um exemplo de um espaço seqüencialmente compacto que não é $\omega$-limitado ([Vau84], exemplo 2.11), pois o conjunto enumerável e infinito $\omega$ não possui fecho compacto em $Y$.

Nosso próximo exemplo será construído a partir de um gap de Hausdorff. Esse exemplo é devido essencialmente a van Douwen ([vD76]).

Exemplo 4.2 Existe um espaço topológico completamente regular que satisfaz o primeiro 
axioma da enumerabilidade, é separável, enumeravelmente paracompacto, coletivamente Hausdorff, localmente compacto e não-normal.

Prova: Sejam $\mathcal{A}_{0}=\left\{A_{0, \xi}: \xi<\omega_{1}\right\}, \mathcal{A}_{1}=\left\{A_{1, \xi}: \xi<\omega_{1}\right\} \subseteq[\omega]^{\omega}$ tais que $\mathcal{A}_{0} \perp \mathcal{A}_{1}, \mathcal{A}_{0}$ e $\mathcal{A}_{1}$ estão bem ordenados por $\subset^{*}$ (e enumerados pelo seu tipo de ordem ) e não podem ser separados; a existência de um par $\left\langle\mathcal{A}_{0}, \mathcal{A}_{1}\right\rangle$ nessas condições foi garantida pelo Teo. 3.5. Considere $L_{i}=\{i\} \times \omega_{1}$ para $i \in 2=\{0,1\}$ e $X=\omega \cup L_{0} \cup L_{1}$. Damos a $X$ a seguinte topologia: os pontos de $\omega$ são declarados isolados e as vizinhanças básicas dos pontos $\langle i, \beta\rangle$ para $i \in 2$ e $\beta<\omega_{1}$ são da forma

$$
B(S, i, \beta, F)=\left\{\langle i, \xi\rangle: S \subset^{*} A_{i, \xi} \subseteq^{*} A_{i, \beta}\right\} \cup\left(A_{i, \beta} \backslash S\right) \backslash F
$$

onde $S \in \mathcal{A}_{i} \cup\{\emptyset\}$ satisfaz $S \subset^{*} A_{i, \beta}$ e $F \in[\omega]^{<\omega}$. Nosso espaço é levemente diferente do de van Douwen, pois na verdade ele considerou $L_{i}=\{i\} \times\left(\omega_{1} \backslash\{0\}\right)$.

Os abertos básicos dados são extremamente análogos aos dados para os pontos não isolados do exemplo anterior; observamos ainda que $(B(S, i, \beta, F) \backslash \omega) \subseteq L_{i}$ para $S, F, i, \beta$ arbitrários. Assim, a topologia está bem definida, e para verificar que $X$ é $T_{2}$ o único caso que não é inteiramente análogo ao exemplo anterior é aquele em que precisamos separar $\langle 0, \zeta\rangle$ de $\langle 1, \xi\rangle$ por abertos disjuntos, para $\zeta, \xi<\omega_{1}$ arbitrários; para isso é só usar que $\mathcal{A}_{0}$ e $\mathcal{A}_{1}$ são ortogonais e portanto $B\left(\emptyset, 0, \zeta,\left(A_{o, \zeta} \cap A_{1, \xi}\right)\right)$ e $B(\emptyset, 1, \xi, \emptyset)$ separam $\langle 0, \zeta\rangle$ e $\langle 1, \xi\rangle$. A verificação de que os abertos básicos que demos são todos compactos é inteiramente análoga àquela que fizemos para o exemplo anterior, e portanto $X$ é localmente compacto. Como compactos em espaços $T_{2}$ são fechados, já está também verificado o fato de $X$ ser zero-dimensional $\left(T_{2}\right)$ e portanto completamente regular. $\omega$ é denso em $X$, já que $A_{i, \beta} \backslash S$ é sempre infinito se $S, i$ e $\beta$ estão nas condições da definição dos abertos básicos, donde $X$ é separável, e é imediato também que $X$ satisfaz o primeiro axioma da enumerabilidade. Verifiquemos então as demais propriedades.

Os subespaços $L_{0}$ e $L_{1}$ de $X$ são centrais nas verificações a serem feitas. Inicialmente, observemos que $L_{0}$ e $L_{1}$ são subespaços fechados disjuntos e que cada um deles é homeomorfo a $\omega_{1}$ com a topologia da ordem; logo, já sabemos que $X$ não é compacto. Mas podemos afirmar que $L_{0}$ e $L_{1}$ são seqüencialmente compactos, logo enumeravelmente compactos, e portanto qualquer cobertura aberta enumerável de $X$ possui uma subcoleção finita que cobre $L_{0} \cup L_{1}$. Mas, os pontos de $\omega$ são isolados. Segue que qualquer cobertura 
aberta enumerável $\mathcal{U}$ de $X$ possui um refinamento aberto localmente finito ( formado por $\mathcal{V} \subseteq \mathcal{U}$ finito que cobre $L_{0} \cup L_{1}$ e pela família $\{\{n\}: n \in X \backslash \cup \mathcal{V}\}$ ), donde $X$ é enumeravelmente paracompacto. $\mathrm{O}$ fato de $L_{0}$ e $L_{1}$ serem fechados e enumeravelmente compactos implica também que, se $D \subseteq X$ é fechado e discreto, então $D \cap L_{i}$ é finito para $i \in 2$ e daí é fácil concluir que $X$ é coletivamente Hausdorff.

Resta mostrar que $X$ não é normal; como o leitor já deve ter "desconfiado", são exatamente $L_{0}$ e $L_{1}$ os fechados disjuntos de $X$ para os quais mostraremos que a existência de abertos disjuntos que os separem leva a uma contradição.

De fato: suponha por absurdo que existam abertos disjuntos $U_{i}$ para $i \in 2$ tais que $L_{i} \subset U_{i}$. Afirmamos que, dado $0<\beta<\omega_{1}$, podemos escolher $0 \leqslant f(\beta)<\beta$ e $E_{\beta} \in[\omega]^{<\omega}$ que não dependem de $i$ (o que é um truque engenhoso de van Douwen) e que satisfazem

$$
B\left(A_{i, f(\beta)}, i, \beta, E_{\beta}\right) \subseteq U_{i} \text { para } i \in 2
$$

Podemos obter $f(\beta)$ e $E_{\beta}$ da seguinte forma: tomamos $A_{0, \zeta}, A_{1, \xi} \operatorname{com} \zeta, \xi<\beta$ e $F, G \in[\omega]^{<\omega}$ tais que $\bar{B}\left(A_{0, \zeta}, 0, \beta, F\right) \subseteq U_{0}, B\left(A_{1, \xi}, 1, \beta, G\right) \subseteq U_{1}$. A partir daí, temos 3 casos: se $\zeta=\xi$, tomamos $f(\beta)=\zeta=\xi$ e $E_{\beta}=F \cup G$; se $\zeta<\xi$, tomamos $f(\beta)=\xi$ e $E_{\beta}=F \cup G \cup\left(A_{0, \zeta} \backslash A_{0, \xi}\right)$; e se $\xi<\zeta, f(\beta)=\zeta$ e $E_{\beta}=F \cup G \cup\left(A_{1, \xi} \backslash A_{1, \zeta}\right)$. Verificações rotineiras mostram que $f(\beta)$ e $E_{\beta}$ assim definidos satisfazem o desejado. Note agora que definimos uma $f: \omega_{1} \backslash\{0\} \mapsto \omega_{1}$ regressiva. Como $\omega_{1}$ é um cardinal regular não enumerável e $\omega_{1} \backslash\{0\}$ é (obviamente!) estacionário em $\omega_{1}$, o Lema de Fodor nos dá que existe $\alpha<\omega_{1}$ tal que $f^{-1}[\{\alpha\}]$ é estacionário em $\omega_{1}$, e portanto podemos afirmar que existe $K \subseteq \omega_{1}$ cofinal e tal que $f(\beta)=\alpha$ para todo $\beta \in K$.

Definimos agora $S_{i} \in[\omega]^{\omega}$ para $i \in 2$ pondo

$$
S_{i}=\left(A_{i, \alpha} \cup\left(\omega \cap U_{i}\right)\right) \backslash A_{1-i, \alpha}
$$

É fácil ver que $S_{0} \cap S_{1}=\emptyset$, já que $U_{0} \cap U_{1}=\emptyset$ e pontos de $A_{i, \alpha}$ não pertencem a $S_{1-i}$. Agora, sejam $\zeta<\omega_{1}$ e $i \in 2$ arbitrários. Como $K$ é cofinal em $\omega_{1}$, existe $\beta \in K$ para o qual $\zeta \leqslant \beta$ e conseqüentemente $A_{i, \zeta} \subseteq^{*} A_{i, \beta}$. Como $f(\beta)=\alpha$, temos $B\left(A_{i, \alpha}, i, \beta, E_{\beta}\right) \subseteq U_{i}$, donde $\left(A_{i, \beta} \backslash A_{i, \alpha}\right) \backslash E_{\beta} \subseteq \omega \cap U_{i}$ e portanto $A_{i, \beta} \subseteq^{*} A_{i, \alpha} \cup\left(\omega \cap U_{i}\right) \subseteq S_{i} \cup A_{1-i, \alpha}$. Mas $A_{i, \beta} \cap A_{1-i, \alpha}$ é finito, logo $A_{i, \beta} \subseteq^{*} S_{i}$ donde $A_{i, \zeta} \subseteq^{*} S_{i}$. Como $i$ e $\zeta$ são arbitrários, teríamos agora que $S_{i}$ separa $\mathcal{A}_{i}$ e $\mathcal{A}_{1-i}$ (já que $A_{i, \zeta} \subseteq S^{*} S_{i}$ e $\left(A_{1-i, \zeta} \cap S_{i}\right) \subseteq\left(A_{1-i, \zeta} \backslash S_{1-i}\right)$ para todo 
$\left.\zeta<\omega_{1}\right)$, o que contradiz a hipótese de $\mathcal{A}_{0}$ e $\mathcal{A}_{1}$ não poderem ser separados. Portanto, $X$ não é normal.

A próxima subseção é dedicada ao estudo da interação existente entre o espaço topológico dado neste último exemplo, a existência de determinados gaps e os cardinais $\mathfrak{p}$ e $\mathfrak{b}$.

\subsubsection{Tight gaps, big gaps, os cardinais $\mathfrak{p}$ e $\mathfrak{b}$ e os espaços de $\left(\omega_{1}, \omega_{1}^{*}\right)$-gaps}

Nesta subseção veremos que a existência de gaps de Hausdorff que satisfazem a determinadas hipóteses adicionais é independente de $Z F C$, no sentido de que conseguiremos fazer com que proposições do tipo " $\mathfrak{p}=\omega_{1}$ " ou " $\mathfrak{b}=\omega_{1}$ " sejam equivalentes à existência desses gaps. No caso do cardinal $\mathfrak{p}$, conseguiremos ainda uma equivalência entre " $\mathfrak{p}=\omega_{1}$ " e a existência de um espaço de gap como no Exemplo 4.2 que, além de enumeravelmente paracompacto, seja também enumeravelmente compacto. Essa discussão foi iniciada por Nyikos e Vaughan ([NV83]) no contexto do que eles denominaram de $R$-espaços: espaços enumeravelmente compactos Hausdorff não-normais, separáveis e que satisfazem o primeiro axioma da enumerabilidade. A questão que se colocava era: é possível exibir um exemplo de R-espaço em $Z F C$ ?

Consideremos um $\left\langle\omega_{1}, \omega_{1}^{*}\right\rangle$-gap $\left\langle\left\{A_{\alpha}: \alpha<\omega_{1}\right\},\left\{B_{\alpha}: \alpha<\omega_{1}\right\}\right\rangle$ em $\left\langle\mathcal{P}(\omega), \subset^{*}\right\rangle$; temos para cada $\alpha<\omega_{1} A_{\alpha} \subset^{*} B_{\alpha}$, logo $B_{\alpha} \backslash A_{\alpha}$ é infinito para todo $\alpha<\omega_{1}$. Podemos imaginar que esse gap "não é muito apertado" se os conjuntos $B_{\alpha} \backslash A_{\alpha}$ (que formam uma subfamília de $[\omega]^{\omega}$ bem-ordenada por $\supseteq^{*}$ ) forem "de tal maneira grandes" a ponto de $\left\{B_{\alpha} \backslash A_{\alpha}: \alpha<\omega_{1}\right\}$ possuir pseudo-intersecções infinitas. Essa "discussão intuitiva" teve o objetivo de tornar um pouco mais natural a introdução das seguintes terminologias.

Definição 4.1 Seja $\left\langle\left\{A_{\alpha}: \alpha<\omega_{1}\right\},\left\{B_{\alpha}: \alpha<\omega_{1}\right\}\right\rangle$ um gap em $\left\langle\mathcal{P}(\omega), \subset^{*}\right\rangle$.

(i) Diremos que um conjunto $E \in[\omega]^{\omega}$ está ao lado do gap se $E \subset^{*} B_{\alpha} \backslash A_{\alpha}$ para todo $\alpha<\omega_{1}$.

(ii) Diremos que o gap em questão é um tight gap se nenhum subconjunto infinito de w está ao lado do gap. 
(iii) Diremos que o gap em questão é um big gap se existir uma família $\left\{E_{n}\right.$ : $n<\omega\} \subseteq[\omega]^{\omega}$ de conjuntos dois-a-dois disjuntos e tais que: (a) cada um dos $E_{n}$ está ao lado do gap; e (b) para cada $D \subseteq \omega$ para o qual $\left|D \cap E_{n}\right|=\omega$ para infinitos $n<\omega$ existe $\alpha<\omega_{1}$ para o qual $\left|A_{\alpha} \cap D\right|=\omega$.

Observamos que a condição " $E \subset^{*} B_{\alpha} \backslash A_{\alpha}$ para todo $\alpha<\omega_{1}$ " pode ser colocada também na seguinte forma: "para todo $\alpha<\omega_{1}, E$ e $A_{\alpha}$ são a.d. e $E \subset \subset^{*} B_{\alpha}$ ". Note portanto que, se $E$ está ao lado do nosso gap, então $A_{\alpha} \nsubseteq^{*} E$ e $E \subset^{*} B_{\alpha}$ para todo $\alpha<\omega_{1}$. Com relação aos big gaps, observamos que se um determinado $E$ está ao lado de um gap, uma decomposição de $E$ na forma $E=\bigcup_{n<\omega} E_{n}$ onde os $E_{n}$ 's são infinitos e disjuntos não pode ser tal que $\left\{E_{n}: n<\omega\right\}$ satisfaça as condições da definição de um big gap ( a exigência $($ iii $)(b)$ não é satisfeita; tome $D=E$ ), logo um big gap é em geral mais do que simplesmente um gap que não é do tipo tight. Reciprocamente, se uma família disjunta $\left\{E_{n}: n<\omega\right\} \subseteq[\omega]^{\omega}$ satisfaz $($ iii $)(a)$ e $(i i i)(b), \bigcup_{n<\omega} E_{n}$ não está ao lado do gap.

Destacaremos agora qual é a "afirmação dual" na linguagem de famílias ortogonais, etc., para a afirmação "determinado gap é do tipo tight". Essa afirmação dual é conseguida por simples passagem ao complementar.

Fato 4.2 Sejam $\mathcal{A}, \mathcal{B} \subseteq[\omega]^{\omega}$ famílias ortogonais, bem-ordenadas por $\subset^{*}$ com tipo de ordem $\omega_{1}$ e que não podem ser separadas. São equivalentes as afirmações:

(i) $\langle\mathcal{A},\{\omega \backslash B: B \in \mathcal{B}\}\rangle$ é um tight gap.

(ii) A família $\left\{\omega \backslash\left(A_{\alpha} \cup B_{\alpha}\right): \alpha<\omega_{1}\right\} \subseteq[\omega]^{\omega}$ não possui pseudo-intersecção infinita.

Já estamos em condições de enunciar um dos principais resultados desta subseção. De agora em diante, chamaremos de espaço de $\left\langle\omega_{1}, \omega_{1}^{\times}\right\rangle$-gap a um espaço construído a partir de um gap de Hausdorff como no Exemplo 4.2. No entanto, como o espaço é construído a partir das famílias ortogonais que não podem ser separadas naturalmente associadas ao gap (conforme Prop. 3.3), alguns pequenos cuidados adicionais deverão ser tomados.

Teorema 4.6 São equivalentes as seguintes afirmações:

(i) Existe um espaço de $\left\langle\omega_{1}, \omega_{1}^{*}\right\rangle$-gap que é enumeravelmente compacto.

(ii) Existe um $\left\langle\omega_{1}, \omega_{1}^{*}\right\rangle$-gap que é um tight gap.

(iii) $\mathfrak{p}=\omega_{1}$. 
Demonstração O Fato 4.2 e a Prop. 3.3 nos dão que a existência de um $\left\langle\omega_{1}, \omega_{1}^{*}\right\rangle$-gap que seja um tight gap implica na existência de uma subfamília de $[\omega]^{\omega}$ bem-ordenada por $\supseteq^{*}$ com tipo de ordem $\omega_{1}$ e sem pseudo-interseç̧ão infinita. Daí $\omega_{1}=\mathfrak{t}_{1}=\mathfrak{t} \geqslant \mathfrak{p} \geqslant \omega_{1}$ e (ii) $\Rightarrow$ (iii) está demonstrado.

$$
\text { Para }(i) \Leftrightarrow(i i) \text {, basta provar o }
$$

Lema 4.1Um $\left\langle\omega_{1}, \omega_{1}^{*}\right\rangle$-gap é um tight gap se e só se o espaço de $\left\langle\omega_{1}, \omega_{1}^{*}\right\rangle$-gap associado a ele é enumeravelmente compacto.

Prova do Lema: Fixemos as notações: $\left\langle\left\{A_{\xi}: \xi<\omega_{1}\right\},\left\{\omega \backslash B_{\xi}: \xi<\omega_{1}\right\}\right\rangle$ é o nosso gap de Hausdorff e as famílias ortogonais, bem-ordenadas por $\subset^{*}$ com tipo de ordem $\omega_{1}$ e que não podem ser separadas a partir das quais construímos nosso espaço de gap são $\left\{A_{\xi}: \xi<\omega_{1}\right\}$ e $\left\{B_{\xi}: \xi<\omega_{1}\right\}$; então $X=\omega \cup L_{0} \cup L_{1}$ é o nosso espaço de gap, construído conforme o Exemplo 4.2 considerando-se $\mathcal{A}_{0}=\left\{A_{\xi}: \xi<\omega_{1}\right\}$ e $\mathcal{A}_{1}=\left\{B_{\xi}: \xi<\omega_{1}\right\}$.

(No restante desta subseção, os espaços considerados serão sempre no mínimo regulares, de modo que para subconjuntos enumeráveis infinitos bastará falarmos em "pontos de acumulação" que estes naturalmente já serão "completos".)

Os subespaços $L_{0}$ e $L_{1}$ de $X$ são enumeravelmente compactos, logo subconjuntos enumeráveis e infinitos de $L_{0} \cup L_{1}$ possuem pontos de acumulação; disso e das expressões para as vizinhanças básicas dos pontos não-isolados podemos afirmar que um subconjunto enumerável e infinito $E^{\prime}$ de $X$ não possui pontos de acumulação se e só se $E^{\prime}={ }^{*} E \in[\omega]^{\omega}$ e $E$ é tal que $E \cap A_{\alpha}$ e $E \cap B_{\alpha}$ são finitos para todo $\alpha<\omega_{1}$, isto é, se e só se $E^{\prime}={ }^{*} E$ e $E$ é uma pseudo-intersecção infinita da família $\left\{\omega \backslash\left(A_{\alpha} \cup B_{\alpha}\right): \alpha<\omega_{1}\right\}$ - ou, mais explicitamente ainda, um subconjunto enumerável e infinito $E^{\prime}$ não possui pontos de acumulação em $X$ se e só se $E^{\prime}={ }^{\times} E$ e $E$ está ao lado do gap ! De fato: o "se" é óbvio e para o "só se", suponha que para um dado $E \in[\omega]^{\omega}$ exista um $\zeta<\omega_{1}$ para o qual $\left|E \cap A_{\zeta}\right|=\omega$; podemos supor s.p.g que tal $\zeta$ é mínimo para essa propriedade. Afirmamos que $\langle 0, \zeta\rangle$ é um ponto de acumulação de $E$. De fato: sejam dados $F \in[\omega]^{<\omega}$ e um $S \in \mathcal{A}_{0} \cup\{\emptyset\}$ que satisfaça $S \subset^{*} A_{\zeta}$; então $S \cap E$ é finito, pela minimalidade de $\zeta$, donde $E \cap B(S, 0, \zeta, F)=E \cap\left(\left(A_{\zeta} \backslash S\right) \backslash F\right)$ que é claramente infinito. Como $F$ e $S$ são arbitrários, $\langle 0, \zeta\rangle$ é um ponto de acumulação de $E$. O mesmo argumento, é claro, 
pode ser feito para o caso em que dado $E \in[\omega]^{\omega}$ existam $\zeta$ 's para os quais $\left|E \cap B_{\zeta}\right|=\omega$. Logo, $X$ possui conjuntos enumeráveis e infinitos sem pontos de acumulação se e só se $\left\{\omega \backslash\left(A_{\alpha} \cup B_{\alpha}\right): \alpha<\omega_{1}\right\}$ possui pseudo-intersecções infinitas; segue do Fato 4.2 que $X$ é enumeravelmente compacto se e só se $\left\langle\left\{A_{\xi}: \xi<\omega_{1}\right\},\left\{\omega \backslash B_{\xi}: \xi<\omega_{1}\right\}\right\rangle$ é um tight gap.

Para $($ iii $) \Rightarrow($ ii $)$, observamos que $\mathfrak{p}=\omega_{1}$ implica $\mathfrak{t}=\omega_{1}$ (item $(b)$ do Teo. 2.1) logo se assumirmos $\mathfrak{p}=\omega_{1}$ existe uma torre $\left\{T_{\alpha}: \alpha<\omega_{1}\right\}$. A partir dessa torre, é possível a construção do tight gap. O mais interessante, porém, é que o fato da família bem ordenada por $\supset^{*}$ e enumerada pelo seu tipo de ordem $\left\{T_{\alpha}: \alpha<\omega_{1}\right\}$ não possuir pseudointersecção infinita não é utilizado na construção do gap; esse fato vai apenas indicar que o gap é do tipo tight. Isso ocorre devido devido ao seguinte resultado, que pode ser obtido mediante pequenas modificações da prova de que existe um gap de Hausdorff (feita neste trabalho no Teo. 3.5) e que é um caso particular de um resultado mais geral (demonstrado de modo um pouco diferente) por Blaszczyk e Szymánski ([BS82]).

Lema 4.2 Se $\left\{T_{\alpha}: \alpha^{-}<\omega_{1}\right\} \subseteq[\omega]^{\omega}$ é uma família de conjuntos coinfinitos que é bemordenada por $\supset^{*}$ (com enumeração dada pelo seu tipo de ordem), então existem famílias $\mathcal{A}=\left\{A_{\alpha}: \alpha<\omega_{1}\right\}, \mathcal{B}=\left\{B_{\alpha}: \alpha<\omega_{1}\right\} \subseteq[\omega]^{\omega}$ tais que $\mathcal{A} \perp \mathcal{B}, \mathcal{A}$ e $\mathcal{B}$ são bem-ordenadas por $\subset^{*}$ com tipo de ordem $\omega_{1}$ e não podem ser separadas e vale ainda que, para todo $\alpha<\omega_{1}, \omega \backslash T_{\alpha}={ }^{*} A_{\alpha} \cup B_{\alpha}$.

Prova do Lema: (esboço) Dada a família $\left\{T_{\alpha}: \alpha<\omega_{1}\right\}$ como no enunciado, são construídos simultaneamente $A_{\alpha}, B_{\alpha} \in[\omega]^{\omega}$ para todo $\alpha<\omega_{1}$ satisfazendo

$$
\begin{aligned}
& \text { (1) } \omega \backslash\left(A_{\alpha} \cup B_{\alpha}\right)={ }^{*} T_{\alpha} \\
& \text { (2) } A_{\alpha} \cap B_{\alpha}=\emptyset \\
& \text { (3) }(\forall \xi<\alpha)\left[\left(A_{\xi} \subset^{*} A_{\alpha}\right) \wedge\left(B_{\xi} \subset^{*} B_{\alpha}\right)\right] \\
& \text { (4) } A_{\alpha}(p)\left\{B_{\xi}: \xi<\alpha\right\}
\end{aligned}
$$

onde, em (4), novamente usamos o conceito de "estar perto de", definido no capítulo sobre gaps. Feita a construção, as justificativas para " $\mathcal{A} \perp \mathcal{B}, \mathcal{A}$ e $\mathcal{B}$ bem-ordenadas por $\subset^{*}$ e não podem ser separadas" são idênticas àquelas feitas na demonstração do Teo. 3.5; a construção é essencialmente a mesma também. Para $\alpha=0$, decompomos $\omega \backslash T_{0}$ em dois conjuntos infinitos e disjuntos, $A_{0}$ e $B_{0}$. Para $0<\delta+1=\alpha<\omega_{1}$, observamos que 
$A_{\delta} \cup B_{\delta}={ }^{*} \omega \backslash T_{\delta} \subset^{*} \omega \backslash T_{\alpha}$ e daí decompomos $\left(\omega \backslash T_{\alpha}\right) \backslash\left(A_{\delta} \cup B_{\delta}\right)$ em dois conjuntos infinitos e disjuntos $C$ e $D$ e fazemos $A_{\alpha}=A_{\delta} \cup C, B_{\alpha}=B_{\delta} \cup D$; para $0<\alpha<\omega_{1}$ limite, o conjunto " $X^{\prime}$ pseudo-intersecção infinita de $\left\{\omega \backslash\left(A_{\xi} \cup B_{\xi}\right): \xi<\alpha\right\}$ " que é tomado na demonstração do Teo. 3.5 pode ser escolhido de modo que $X^{\prime}=T_{\alpha}$. Seguindo-se a mesma notação usada no teorema tantas vezes já citado, teríamos $X=\omega \backslash T_{\alpha}, A_{\alpha} \subseteq X$ e $B_{\alpha}=X \backslash A_{\alpha}$, logo a construção satisfaz a todas as condições desejadas e o Lema está demonstrado.

Com o Lema, (iii) $\Rightarrow$ (ii) segue, pois, como já havíamos comentado, $\mathfrak{p}=\omega_{1}$ nos permite supor que a família $\left\{T_{\alpha}: \alpha<\omega_{1}\right\}$ tomada no Lema não possui pseudo-intersecção infinita, conseqüentemente as famílias $\mathcal{A}$ e $\mathcal{B}$ construídas são tais que $\left\{\omega \backslash\left(A_{\alpha} \cup B_{\alpha}\right)\right.$ : $\left.\alpha<\omega_{1}\right\}$ não possui pseudo-intersecção infinita e já sabemos que isso implica que o gap associado a $\mathcal{A}$ e $\mathcal{B}$ é do tipo tight.

Observamos que em qualquer modelo de $M A+\neg C H$ tem-se $\mathfrak{p}=\mathfrak{c}>\omega_{1}$, logo sob $M A+\neg C H$ não existêm espaços de $\left\langle\omega_{1}, \omega_{1}^{*}\right\rangle$-gaps que sejam enumeravelmente compactos. Por outro lado, o Teorema nos mostra que em determinados modelos de $Z F C$ (por exemplo, sob $\mathrm{CH}$ ) existem $R$-espaços.

Temos ainda o seguinte

Corolário São equivalentes as afirmações:

(i) Existe um $\left\langle\omega_{1}, \omega_{1}^{*}\right\rangle$-gap em $\left\langle\mathcal{P}(\omega), \subset^{*}\right\rangle$ que é do tipo tight.

(ii) Existe um $\left\langle\omega_{1}, 1^{*}\right\rangle$-gap em $\left\langle\mathcal{P}(\omega), \subset^{*}\right\rangle$.

Demonstração Sabemos que (ii) é equivalente a $t=\omega_{1}$, conforme observação feita após a demonstração da proposição 3.4. Por outro lado, o Teorema 4.6 nos dá $\mathfrak{p}=\omega_{1}$ equivalente a (i). Mas, $\mathfrak{p}=\omega_{1} \Leftrightarrow \mathfrak{t}=\omega_{1}$ (item (b) do Teo. 2.1).

Tratemos agora dos big gaps. O seguinte teorema nos dá uma "justificativa topológica" para a escolha do termo "big gap". A questão ainda se relaciona com os Respaços da seguinte maneira: suponha que um espaço de gap $X=\omega \cup L_{0} \cup L_{1}$ seja imerso num espaço de Hausdorff $Y$ que satisfaça o primeiro axioma da enumerabilidade; então, como tal $Y$ é seqüencial, seus subespaços enumeravelmente compactos são fechados, em particular $L_{0}$ e $L_{1}$ são fechados (em $Y$ ) e portanto $Y$ não é normal. Tal fato sugere uma 
maneira de se obter R-espaços: basta imergir um espaço de gap num espaço $Y$, enumeravelmente compacto Hausdorff que satisfaça o primeiro axioma da enumerabilidade: se uma tal imersão existir, basta tomarmos o fecho (da imagem) do espaço de gap em tal espaço $Y$ e teremos que esse fecho será enumeravelmente compacto, $T_{2}$, separável, primeiro enumerável e não-normal, isto é , o fecho será um R-espaço. Nossa argumentação sobre o fecho da imagem da imersão pode ser resumida da seguinte forma: se existe uma imersão de um espaço de gap $X$ num espaço de Hausdorff enumeravelmente compacto $Y$ que satisfaz o primeiro axioma da enumerabilidade, podemos supor que a imersão é densa e nessas condições $Y$ é um R-espaço. Porém, isso não será possível para espaços de gaps que provenham de big gaps, pois vale o

Teorema 4.7 Sejam $\mathcal{A}_{0}, \mathcal{A}_{1} \subseteq[\omega]^{\omega}$ tais que $\mathcal{A}_{0} \perp \mathcal{A}_{1}, \mathcal{A}_{0}$ e $\mathcal{A}_{1}$ são bem-ordenadas por $\subset^{*}$ com tipo de ordem $\omega_{1}$ e não podem ser separadas e tais que o $\left\langle\omega_{1}, \omega_{1}^{\times}\right\rangle$-gap associado seja um big gap. Então o espaço de gap $X=\omega \cup L_{0} \cup L_{1}$ construído a partir de $\mathcal{A}_{0}$ e $\mathcal{A}_{1}$ não pode ser imerso num espaço de Hausdorff enumeravelmente compacto que satisfaça o primeiro axioma da enumerabilidade.

Demonstração : Aplicada a $\mathcal{A}_{0}$ e $\mathcal{A}_{1}$, a condição "o gap é um big gap" implica na existência de uma família disjunta $\left\{E_{n}: n<\omega\right\} \subseteq[\omega]^{\omega}$ tal que cada um dos $E_{n}$ é pseudo-intersecção infinita da família $\left\{\omega \backslash\left(A_{0, \xi} \cup B_{0, \xi}\right): \xi<\omega_{1}\right\}$ (logo, cada um dos $E_{n}$ não possui pontos de acumulação em $X$, conforme já observamos) e vale ainda que, se $D \subseteq \omega$ é tal que $\left|D \cap E_{n}\right|=\omega$ para infinitos $n<\omega$, então existe $\alpha<\omega_{1}$ para o qual $\left|A_{0, \alpha} \cap D\right|=\omega$. Pois bem: suponha por absurdo que o espaço de gap $X=\omega \cup L_{0} \cup L_{1}$ seja homeomorfo a um subespaço de um espaço $Y, T_{2}$, enumeravelmente compacto e que satisfaça o primeiro axioma da enumerabilidade: por comodidade, assumamos que $X \subseteq Y$, e, como já observamos, podemos assumir também que a imersão é densa. Cada um dos $E_{n}$ não possui pontos de acumulação em $X$; como $Y$ é enumeravelmente compacto, cada $E_{n}$ possui um ponto de acumulação $y_{n} \in Y \backslash X$; passando para subseqüencias, se necessário - o que é possível pois $Y$ é seqüencial, e nessa classe de espaços os enumeravelmente compactos são seqüencialmente compactos -, podemos supor que cada $E_{n}$ converge para $y_{n}$. O conjunto $\left\{y_{n}: n<\omega\right\}$ possui um ponto de acumulação $y$, e novamente por passagem a subseqüências podemos supor que $\left\langle y_{n}: n<\omega\right\rangle$ converge para $y$; se $\left\{y_{n}: n<\omega\right\}$ for um conjunto finito, podemos assumir que $y$ é o limite de uma subseqüencia constante 
$\left\langle y_{n_{k}}: k<\omega\right\rangle$ e toda nossa argumentação a seguir continua valendo. Tal ponto $y$, limite de $\left\langle y_{n}: n<\omega\right\rangle$, não pertence a $X$, pois $X$ é localmente compacto e denso no espaço $T_{2} Y$ e portanto $Y \backslash X$ é fechado ([Eng89],3.3.9). Seja agora $\left\{U_{n}: n<\omega\right\}$ uma base local para $y$ em $Y$, que podemos supor decrescente. Tomamos agora uma seqüência estritamente crescente de naturais $\left\{i_{n}: n<\omega\right\}$ de modo que para todo $n<\omega$ tenhamos $D_{i_{n}}=U_{n} \cap E_{i_{n}}$ infinito, o que é possível dentro das suposições feitas. Definindo agora $D=\bigcup_{n<\omega} D_{i_{n}}$, pela hipótese do gap ser um big gap existe $\alpha<\omega_{1}$ tal que $\left|D \cap A_{0, \alpha}\right|=\omega \mathrm{e}$ nessas condições sabemos que $D$ possui um ponto de acumulação em $L_{0} \subseteq X$, o que é um absurdo pois $D$ é tal que todos os seus pontos de acumulação não estão em $X$ (de fato: suponha por absurdo que $D$ possui um ponto de acumulação $x \in X$; posso supor s.p.g. que existe $N \subseteq D$ tal que $N$ converge para $x$. Então $N \cap D_{i_{n}}$ é finito para todo $n<\omega$, caso contrário algum dos $y_{k}$ seria ponto de acumulação de $N$; mas $N \cap D_{i_{n}}$ finito para todo $n<\omega$ faz com que $N$ convirja para $y \notin X$; como o espaço é $T_{2}$, os limites devem ser únicos e isto é uma contradição). Assim sendo, supor que $X$ pode ser imerso num espaço enumeravelmente compacto $T_{2}$ que satisfaz o primeiro axioma da enumerabilidade resultou num absurdo.

O próximo teorema dá uma equivalência entre a existência de big gaps e a igualdade $\mathfrak{b}=\omega_{1}$.

Teorema 4.8 São equivalentes as afirmações:

(i) Existe um $\left\langle\omega_{1}, \omega_{1}^{*}\right\rangle$-gap que é um big gap.

(ii) $\mathfrak{b}=\omega_{1}$.

Demonstração Prova de $(i) \Rightarrow($ ii $)$ : Seja $\left\langle\left\{A_{\alpha}: \alpha<\omega_{1}\right\},\left\{B_{\alpha}: \alpha<\omega_{1}\right\}\right\rangle$ um big gap em $\left\langle\mathcal{P}(\omega), \subset^{*}\right\rangle$ e seja $\left\{E_{n}: n<\omega\right\}$ uma família disjunta de subconjuntos infinitos de $\omega$ que faz com que o gap satisfaça a definição de big gap. Para cada $n<\omega$ e $\alpha<\omega_{1}$ temos $E_{n} \subset^{*} B_{\alpha} \backslash A_{\alpha}$, logo é possível definir uma família de funções $\left\{f_{\alpha}: \alpha<\omega_{1}\right\} \subseteq{ }^{\omega} \omega$ pondo

$$
f_{\alpha}(n)=\min \left\{k<\omega:(\forall j<\omega)\left[j \geqslant k \Rightarrow\left(j \in E_{n} \Rightarrow j \in B_{\alpha} \backslash A_{\alpha}\right)\right]\right\}
$$

Afirmamos que $\left\{f_{\alpha}: \alpha<\omega_{1}\right\}$ é ilimitada, donde segue o desejado. Suponha por absurdo que exista $g \in{ }^{\omega} \omega$ para a qual $f_{\alpha} \leqslant{ }^{*} g$ para todo $\alpha<\omega_{1}$. Definimos então $D_{n}=\left\{m \in E_{n}: m>g(n)\right\} ; D_{n}$ é um subconjunto infinito de $E_{n}$ para todo $n<\omega$, 
e da suposição de que o gap é um big gap existe um determinado $\alpha<\omega_{1}$ para o qual $\left|A_{\alpha} \cap\left(\bigcup_{n<\omega} D_{n}\right)\right|=\omega$. Porém, $D_{n} \subseteq E_{n}$ nos dá $\left|D_{n} \cap A_{\alpha}\right|<\omega$ para todo $n<\omega$, dessa forma existem infinitos $n<\omega$ para os quais $D_{n} \cap A_{\alpha} \neq \emptyset$. Como $f_{\alpha} \leqslant *$, existe $k<\omega$ tal que para todo $m \geqslant k$ tem-se $f_{\alpha}(m) \leqslant g(m)$. Tomemos agora um $n_{0}<\omega$ tal que $n_{0}>k$ e existe $j \in D_{n_{0}} \cap A_{\alpha}$; temos $j>g\left(n_{0}\right) \geqslant f_{\alpha}\left(n_{0}\right)$ e a definição da $f_{\alpha}$ nos dá $j \in B_{\alpha} \backslash A_{\alpha}$, o que contradiz $j \in A_{\alpha}$. Portanto, $\left\{f_{\alpha}: \alpha<\omega_{1}\right\}$ é ilimitada e $\mathfrak{b}=\omega_{1}$.

Prova de $($ ii $) \Rightarrow(i)$ : Assumindo $\mathfrak{b}=\omega_{1}$, mostraremos que existe um big gap em $\langle\mathcal{P}(M)$, $\left.\complement^{*}\right\rangle$ para algum $M$ infinito e enumerável, o que é suficiente para nós. Sejam então: $N$ um conjunto enumerável, infinito e disjunto de $\omega \times \omega ;\left\langle\left\{A_{\alpha}^{\prime}: \alpha<\omega_{1}\right\},\left\{B_{\alpha}^{\prime}: \alpha<\omega_{1}\right\}\right\rangle$ $\operatorname{um}\left\langle\omega_{1}, \omega_{1}^{*}\right\rangle$-gap em $\left\langle\mathcal{P}(N), \subset^{*}\right\rangle$; e $\left\{f_{\alpha}: \alpha<\omega_{1}\right\} \subseteq{ }^{\omega} \omega$ uma família ilimitada de funções estritamente crescentes bem-ordenada por $<^{*}$ (usando aqui a igualdade $\mathfrak{b}=\mathfrak{b}_{1}$ do Teo. 2.2). Definimos então $A_{\alpha}=A_{\alpha}^{\prime} \cup\left\{\langle i, j\rangle \in \omega \times \omega: j \leqslant f_{\alpha}(i)\right\}=A_{\alpha}^{\prime} \cup L_{f_{\alpha}}$ (onde as $L_{f}$ para $f \in{ }^{\omega} \omega$ são definidas na prova do Teo. 2.2) e $B_{\alpha}=B_{\alpha}^{\prime} \cup(\omega \times \omega)$. Afirmamos que $\left\langle\left\{A_{\alpha}: \alpha<\omega_{1}\right\},\left\{B_{\alpha}: \alpha<\omega_{1}\right\}\right\rangle$ é um big gap em $(\omega \times \omega) \cup N$. Como " $L_{f} \subset^{*} L_{g} \Leftrightarrow f<{ }^{*} g$ " vale para quaisquer $f, g \in{ }^{\omega} \omega$ (Teo. 2.2 de novo), o fato de $\left\langle\left\{A_{\alpha}: \alpha<\omega_{1}\right\},\left\{B_{\alpha}: \alpha<\omega_{1}\right\}\right\rangle$ ser um gap é imediato. Afirmamos agora que, se fizermos $E_{k}=\{k\} \times \omega$ para todo $k<\omega$ teremos que $\left\{E_{k}: k<\omega\right\}$ satisfaz as condições necessárias para que o gap seja do tipo big gap (note que $\left\{E_{k}: k<\omega\right\}=\mathcal{V}$, na notação do Teo. 2.2). A condição $E_{k} \subset^{*} B_{\alpha} \backslash A_{\alpha}$ para todo $k<\omega$ e para todo $\alpha<\omega_{1}$ é óbvia. Suponha agora que $D \subseteq(\omega \times \omega) \cup N$ seja tal que $\left|D \cap E_{k}\right|=\omega$ para infinitos $n<\omega$; então, em particular, existe $I \subseteq \omega$ infinito para o qual $D \cap E_{i} \neq \emptyset$ para todo $i \in I$ e podemos definir $f \in{ }^{\omega} \omega$ pondo $f(n)=0$ se $n \notin I$ e $f(i)=\min \{j<\omega:\langle i, j\rangle \in D\}$ para todo $i \in I$. Como $\left\{f_{\alpha}: \alpha<\omega_{1}\right\}$ é família ilimitada de funções estritamente crescentes, tal família é ilimitada em $I$ (Fato 2.5) e portanto existe um determinado $\alpha<\omega_{1}$ para o qual $\left\{i \in I: f(i)<f_{\alpha}(i)\right\}$ é infinito; daí $D \cap A_{\alpha}$ é infinito (pois $f\left\lceil I\right.$ é subconjunto infinito de $D$ e de $A_{\alpha}$ ) e mostramos o desejado.

Os R-espaços (que de certa forma motivam o estudo dos espaços de gaps que fizemos nesta subseção) são exemplos de espaços de Hausdorff enumeravelmente compactos, primeiro enumeráveis, separáveis e não-compactos; atualmente, a exigência "nãocompacto" tem sido mais freqüente do que a exigência "não-normal" (ver, por exemplo, o artigo de Nyikos ([Nyi90]) no Open Problems), o que explica o relativo "desuso" da ex- 
pressão "R-espaço". Por exemplo, em modelos nos quais vale " $t=\omega_{1}$ " o espaço discutido no Exemplo 4.1 é seqüencialmente compacto, logo enumeravelmente compacto, satisfaz o primeiro axioma da enumerabilidade, é separável, Hausdorff e não-compacto; porém, é normal. Mais ainda: no artigo de Nyikos citado ([Nyi90] ,p.133, 2.1 a 2.3), vemos que uma construção do tipo $\gamma \mathbb{I N}$ - símbolo genérico dado a um espaço de Hausdorff $X$ localmente compacto no qual $\mathbb{N}$ é um conjunto denso de pontos isolados e $X \backslash \mathbb{N}$ é homeomorfo a $\omega_{1}$ com a topologia da ordem - é enumeravelmente compacta se e só se $\mathfrak{t}=\omega_{1}$.

Não se conhecem ainda em " $Z F C$ puro" exemplos de espaços $T_{2}$ enumeravelmente compactos, primeiro enumeráveis, separáveis e não-compactos. Pode até ser consistente que um tal espaço não exista (na última seção de [Nyi90], dramaticamente intitulada de "Terra de Ninguém", pergunta-se sobre a existência de um tal espaço num determinado modelo de $Z F C$, devido a Alan Dow, no qual $\omega_{1}<\mathfrak{p}$ e $\mathfrak{b}<\mathfrak{o}$.).

\subsubsection{FR-espaços e alguns resultados de Nyikos e Vaughan}

Conforme observado na terceira subseção desta seção, dizemos um um espaço topológico $X$ é um $F R$-espaço se $X$ for um espaço de Hausdorff localmente compacto que possui um subconjunto discreto denso e enumerável cujo complementar é homeomorfo a um ordinal com a topologia da ordem. Nosso primeiro exemplo de FR-espaço foi o Exemplo 4.1, construído a partir dos complementares de uma torre de tamanho mínimo; tal espaço é seqüencialmente compacto e não-compacto. O artigo [NV87] de Nyikos e Vaughan dá uma caracterização dos FR-espaços seqüencialmente compactos não-compactos a partir de espaços construídos essencialmente como no Exemplo que citamos.

Vejamos como se dá essa caracterização. Seja $\mathcal{T}=\left\{T_{\alpha}: \alpha<\delta\right\}$ uma torre; assumiremos s.p.g. que $\omega \backslash T_{0}$ é infinito ( o que pode ser feito tranqüilamente, conforme comentamos na demonstração do Teo. 2.4 ). Consideramos $W$ o espaço dado por $\delta$ com a topologia da ordem, mas assumindo $W \cap \omega=\emptyset$, e fazemos $X=\omega \cup W$. Para cada $\alpha \in W \backslash\{0\}$, definimos vizinhanças básicas para $\alpha$ da forma

$$
B(\beta, \alpha, F)=\{\gamma \in W: \beta<\gamma \leqslant \alpha\} \cup\left(T_{\beta} \backslash T_{\alpha}\right) \backslash F
$$

para todos $\beta<\alpha$ e $F \in[\omega]^{<\omega}$. Para $\alpha=0 \in W$, definimos as vizinhanças básicas $B(0, F)=\{0\} \cup\left(\omega \backslash T_{0}\right) \backslash F$ para todo $F \in[\omega]^{<\omega}$. É fácil ver que, essencialmente, esta 
topologia "comporta-se" como a topologia do Exemplo 4.1; segue que $X=X(\mathcal{T})$ é um FR-espaço seqüencialmente compacto (já que $c f(\delta)>\omega$ e $\mathcal{T}$ não possui pseudo-intersecção infinita) e não-compacto (pois $\delta$ é ordinal limite e $W$ é subespaço fechado de $X$ ).

Por outro lado, se $X$ é um FR-espaço, identificaremos seu subconjunto discreto denso e enumerável com $\omega$ e assumiremos que $W=X \backslash \omega$ é homeomorfo a um ordinal $\delta$ com a topologia da ordem, com, é claro, $W \cap \omega=\emptyset$.

Temos então o

Teorema 4.9 Para toda torre $\mathcal{T} \subseteq[\omega]^{\omega}$ existe um FR-espaço seqüencialmente compacto e não-compacto $X=X(\mathcal{T})$. Reciprocamente, se $X$ é um FR-espaço seqüencialmente compacto e não-compacto, existe uma torre $\mathcal{T} \subseteq[\omega]^{\omega}$ tal que $X=X(\mathcal{T})$.

Demonstração Tanto a construção de $X=X(\mathcal{T})$ a partir de uma torre $\mathcal{T}$ quanto a verificação de que tal $X$ é um FR-espaço seqüencialmente compacto não-compacto já foram feitas; resta demonstrar a recíproca. Seja então $X=\omega \cup W$ um FR-espaço seqüencialmente compacto não-compacto. Afirmamos que para todo $\alpha \in X$ existe um subespaço aberto-fechado $U_{\alpha} \subseteq X$ tal que $U_{\alpha}$ é compacto e $U_{\alpha} \cap W=[0, \alpha]$. De fato: fixemos $\alpha \in W$ e consideremos os abertos-fechados de $W$ dados por $[0, \alpha]$ e $[\alpha+1, \delta[$. De $W$ subespaço fechado de $X$, da regularidade de $X$ (que é localmente compacto) e da compacidade de $[0, \alpha]$ é fácil construir abertos disjuntos $U_{\alpha}$ e $V$ tais que $[0, \alpha] \subset U_{\alpha}$ e $\left[\alpha+1, \delta\left[\subset V\right.\right.$; é imediato agora que $U_{\alpha}$ e $V$ são fechados, sendo portanto clopens seqüencialmente compactos, e que $U_{\alpha} \cap W=[0, \alpha]$. Para verificarmos que $U_{\alpha}$ é compacto, basta provarmos que todo subconjunto infinito de $U_{\alpha}$ possui um ponto de acumulação completo em $U_{\alpha}$. Pois bem: seja $A \subseteq U_{\alpha}$ infinito. Se $|A|=\omega$, não há o que provar. Se $|A|>\omega$, podemos supor s.p.g. que $A \subseteq[0, \alpha]$ e a compacidade de $[0, \alpha]$ liqüida a questão.

Temos então uma família de clopens compactos $\left\{U_{\alpha}: \alpha \in W\right\}$ satisfazendo $U_{\alpha} \cap$ $W=[0, \alpha]$ para todo $\alpha \in W$. Observamos que, se $\alpha, \beta \in W, \alpha<\beta$, então o fechado $U_{\alpha} \backslash U_{\beta}$ é forçosamente um subconjunto finito de $\omega$ (caso contrário, $U_{\alpha} \backslash U_{\beta}$ teria um ponto de acumulação em $\left(U_{\alpha} \backslash U_{\beta}\right) \cap W$, que é vazio), donde segue $U_{\alpha} \subset^{*} U_{\beta}$. Considere então $T_{\alpha}=\omega \backslash U_{\alpha}$ para cada $\alpha \in W$; cada $T_{\alpha}$ é infinito, pois $X \backslash U_{\alpha}$ é aberto, logo $\left\{T_{\alpha}: \alpha \in W\right\}$ é uma subfamília de $[\omega]^{\omega}$ bem-ordenada por $\supset^{*}$. Seja agora $I$ uma pseudo-intersecção de $\left\{T_{\alpha}: \alpha \in W\right\}$; verificaremos que $I$ é finito, o que garantirá que $\mathcal{T}=\left\{T_{\alpha}: \alpha \in \mathcal{G}^{\prime} W\right\}$ é uma torre. De fato: um tal $I$ deve satisfazer $\left|I \cap U_{\alpha}\right|<\omega$ para todo $\alpha \in W$, logo $I$ 
é finito caso contrário possuiria um ponto de acumulação $\beta \in W$ ( $X$ é seqüencialmente compacto) para o qual valeria $\left|I \cap U_{\beta}\right|=\omega$. Mostraremos agora que $X=X(\mathcal{T})$, isto é, a topologia original de $X$ coincide com a topologia que se obtém a partir da torre $\mathcal{T}$. Isso encerrará a demonstração.

O conjunto-suporte de ambas as topologias é o mesmo: $X=\omega \cup W$. Seja $\tau_{1}$ a topologia original de $X$ e $\tau_{2}$ a topologia de $X(\mathcal{T})$. Para $\tau_{2} \subseteq \tau_{1}$, basta observar que todos os abertos básicos de $X(\mathcal{T})$ são abertos de $X$; de fato, se $\beta<\alpha \in W$ e $F \in[\omega]^{<\omega}$ temos $B(\beta, \alpha, F)=] \beta, \alpha] \cup\left(T_{\beta} \backslash T_{\alpha}\right) \backslash F=\left(U_{\alpha} \backslash U_{\beta}\right) \backslash F=U_{\alpha} \backslash\left(U_{\beta} \cup F\right)$ que é aberto, e analogamente para $\alpha=0$. Para $\tau_{1} \subseteq \tau_{2}$ seja $U$ um aberto de $X$ e $\alpha \in W \cap U, \alpha \neq 0$; existe $\beta<\alpha$ para o qual $] \beta, \alpha] \subset U$, logo $\left(U_{\alpha} \backslash U_{\beta}\right) \backslash U$ deve ser finito caso contrário possuiria um ponto de acumulação em $] \beta, \alpha]$. Assim, fazendo $F=\left(U_{\alpha} \backslash U_{\beta}\right) \backslash U$, temos $U \supset] \beta, \alpha] \cup\left(U_{\alpha} \backslash U_{\beta}\right) \backslash F=\{\gamma \in W: \beta<\gamma \leqslant \alpha\} \cup\left(T_{\beta} \backslash T_{\alpha}\right) \backslash F=B(\beta, \alpha, F)$; para $\alpha=0$, um argumento semelhante pode ser dado, logo $U$ é aberto em $\tau_{2}$ e as topologias coincidem.

É interessante observarmos que, na demonstração dada, se $U_{\alpha}^{\prime}$ é uma outra escolha de clopen compacto satisfazendo $U_{\alpha}^{\prime} \cap W=[0, \alpha]$, argumentos análogos aos dados nos dão $U_{\alpha} \backslash U_{\alpha}^{\prime}$ e $U_{\alpha}^{\prime} \backslash U_{\alpha}$ finitos; assim $U_{\alpha}={ }^{*} U_{\alpha}^{\prime}$ e a torre $\mathcal{T}$ obtida tal que $X=X(\mathcal{T})$ é essencialmente única.

O próximo resultado a ser apresentado requer algumas definições.

Definição 4.2 Seja $u \in \omega^{*}=\beta \omega \backslash \omega$ (isto é, u é um ultrafiltro não-principal em $\omega$ ) $e$ $f: \omega \mapsto X$ uma sequência num espaço $X$. Dizemos que um ponto $x \in X$ é um $u$-limite de $f$ se para toda vizinhança $V$ de $x$ tivermos $f^{-1}[V] \in u$.

Observamos que, se $X$ é um espaço $T_{2}$ e $u$ é um ultrafiltro não-principal, então uma sequência $X$ tem no máximo um $u$-limite. Logo, para espaços de Hausdorff podemos dizer um ponto $x, u$-limite de uma seqüência $f$ é $o u$-limite de $f$ e denotá-lo por $x=u-\lim f$.

Sejam então $X$ um espaço de Hausdorff e $f: \omega \mapsto X$ uma sequência; se $x \in X$ é um ponto de acumulação da sequência, é fácil (embora tedioso) construir um ultrafiltro não-principal $u$ tal que $x=u-\lim f$. Por outro lado, se existe $u \in \omega^{*}$ tal que $x=u-\lim$ $f$, o fato de que ultrafiltros não-principais possuem como elementos apenas..subconjuntos infinitos de $\omega$ garante que $x$ é ponto de acumulação da seqüência. Vale então o seguinte 
Fato 4.3 Seja $X$ um espaço $T_{2}$ e $f: \omega \mapsto X$ uma sequência. Então $x \in X$ é ponto de acumulação de $f$ se e só se existe $u \in \omega^{*}$ tal que $x=u-\lim f$.

Se um espaço de Hausdorff $X$ é enumeravelmente compacto, toda sequência $f$ possui um $u$-limite para algum $u \in \omega^{*}$; porém, sequências diferentes podem exigir ultrafiltros diferentes. Isso motiva a

Definição 4.3 Seja $u \in \omega^{*}$. Um espaço $X$ é dito u-compacto se toda sequência em $X$ possui um u-limite em $X$.

É claro, portanto, que se existir $u \in \omega^{*}$ para o qual um espaço $X$ é $u$-compacto, tal espaço é enumeravelmente compacto; porém, a recíproca não é verdadeira, isto é, é possível exibir um espaço $X$ enumeravelmente compacto para o qual não existe $u \in \omega^{*}$ tal que $X$ é $u$-compacto ([Vau84], ex.4.8; a chave para este exemplo é que a $u$-compacidade é produtiva, enquanto que a compacidade enumerável não é.).

Uma última definição e já enunciaremos o próximo resultado.

Definição 4.4 Um ultrafiltro $u \in \beta \omega$ é dito um $T$-ponto se existir uma torre $\mathcal{T}$ tal que $\mathcal{T} \subset u$.

Teorema 4.10 Seja dado $X=X(\mathcal{T})$ um FR-espaço seqüencialmente compacto e nãocompacto e $u \in \omega^{*}$. Então $X$ não é u-compacto se e só se existe um ultrafiltro $v$ tal que $\mathcal{T} \subset v$ e $v \leqslant_{R K} u$.

Relembramos ao leitor que a desigualdade " $\leqslant R K$ " que aparece no enunciado do Teorema é com relação à pré-ordem de Rudin-Keisler em $\beta \omega: v \leqslant_{R K} u$ significa que existe $f \in{ }^{\omega} \omega$ tal que $\bar{f}(u)=\left\{A \subseteq \omega: f^{-1}[A] \in u\right\}=v$. Note que, dados $u$ ultrafiltro e $g \in{ }^{\omega} \omega$ quaisquer, $\bar{g}(u)$ é ultrafiltro.

Demonstração Inicialmente assumamos que, dados $u \in \omega^{*}$ e $X=X(\mathcal{T})$, exista $f \in{ }^{\omega} \omega$ tal que $\mathcal{T} \subset \bar{f}(u) \in \beta \omega$; exibiremos uma sequência $g: \omega \mapsto X$ que não possui $u$-limites, donde $X=X(\mathcal{T})$ não é $u$-compacto. Tomemos $g$ a função que se obtém olhando-se $f$ como uma função com contradomínio $\omega \cup W=X$; então, para todo $\alpha \in W,[0, \alpha] \cup\left(\omega \backslash T_{\alpha}\right)$ é uma vizinhança (aberta-fechada) de $\alpha$ para a qual 


$$
g^{-1}\left[[0, \alpha] \cup\left(\omega \backslash T_{\alpha}\right)\right]=g^{-1}\left[\omega \backslash T_{\alpha}\right]=f^{-1}\left[\omega \backslash T_{\alpha}\right] \notin u
$$

já que $\mathcal{T} \subset \bar{f}(u)$ implica $f^{-1}\left[T_{\alpha}\right] \in u$ para todo $\alpha \in W$. Assim, $X$ não é $u$-compacto.

Reciprocamente, sejam $X=X(\mathcal{T})$ e $u \in \omega^{*}$ tais que $X$ não é $u$-compacto, e seja $g: \omega \mapsto X$ uma sequência que não possua $u$-limites em $X$. Definimos $f \in{ }^{\omega} \omega$ pondo

$$
f(n)= \begin{cases}g(n) \quad \text { se } g(n) \in \omega \\ 0 \in \omega & \text { se } g(n) \in W\end{cases}
$$

Mostraremos que $\mathcal{T} \subset \bar{f}(u)$, o que garante o desejado. Pois bem: para todo $\alpha \in W$ seja $K_{\alpha}=\left([0, \alpha] \cup\left(\omega \backslash T_{\alpha}\right)\right) \cup \overline{(g[\omega] \cap W)}^{X}$; temos que $K_{\alpha}$ é compacto. Assim, cobrindo $K_{\alpha}$ por abertos $V$ que satisfazem $g^{-1}[V] \notin u$, concluímos que $g^{-1}\left[K_{\alpha}\right] \notin u$ (basta lembrar que, se uma união finita pertence a um ultrafiltro, então algum dos conjuntos unidos pertence ao ultrafiltro). Assim, para todo $\alpha \in W, f^{-1}\left[\omega \backslash T_{\alpha}\right] \notin u$, pois $f^{-1}\left[\omega \backslash T_{\alpha}\right] \subseteq g^{-1}\left[K_{\alpha}\right] \notin u$. Portanto $f^{-1}\left[T_{\alpha}\right] \in u$ para todo $\alpha \in W$ e $\mathcal{T} \subset \bar{f}(u)$, conforme desejado.

O Teorema que acabamos de apresentar tem um interessante corolário relacionado ao Problema de Scarborough e Stone. É sabido que, se $\left\{X_{\alpha}: \alpha \in I\right\}$ é uma família de espaços topológicos, então $X=\prod_{\alpha \in I} X_{\alpha}$ é enumeravelmente compacto se e só se para toda sequência $f: \omega \mapsto X$ existe $u \in \omega^{*}$ para o qual $\prod_{\alpha} \circ f$ possui $u$-limite em $X_{\alpha}$ para todo $\alpha \in I$ ([Vau84] 4.4). Segue que, se $X=\prod_{\alpha \in I} X_{\alpha}$ não é enumeravelmente compacto, então para todo $u \in \omega^{*}$ existe $\alpha \in I$ tal que $X_{\alpha}$ não é $u$-compacto; segue também que, se $\left\{X_{u}: u \in \omega^{*}\right\}$ é uma família de espaços topológicos satisfazendo " $X_{u}$ não é $u$-compacto" para todo $u \in \omega^{*}$, então $\prod_{u \in \omega^{*}} X_{u}$ não é enumeravelmente compacto ([NV92],3.2; a sequência contra-exemplo é simplesmente a função diagonal de uma família $\left\{f_{u}: u \in \omega^{*}\right\}$ que satisfaz " $f_{u}: \omega \mapsto X_{u}$ não possui $u$-limite em $X_{u}$ " para todo $u \in \omega^{*}$.). Vale portanto o seguinte fato, reconhecido atualmente ([NV92]) como "estratégia padrão" para a procura de soluções negativas do Problema de Scarborough e Stone:

Fato 4.4 Existe uma família de espaços seqüencialmente compactos cujo produto não é enumeravelmente compacto se e só se para todo $u \in \omega^{*}$ existe um espaço seqüencialmente compacto $X$ tal que $X$ não é u-compacto. 
O corolário a seguir é uma "restrição" do Fato 4.4 para a classe dos FR-espaços seqüencialmente compactos não-compactos, usando o que foi provado no Teorema 4.10.

Corolário Existe uma famíia de FR-espaços seqüencialmente compactos cujo produto não é enumeravelmente compacto se e só se para todo $u \in \omega^{*}$ existe um $T$-ponto $v$ para o qual $v \leqslant_{R K} u$.

Usando um modelo de $Z F C$ construído por Balcar, Frankiewicz e Mills no qual todo $u \in \omega^{*}$ é um $T$-ponto (e que $u \leqslant_{R K} u$ vale para qualquer ultrafiltro), Nyikos e Vaughan estabeleceram no artigo já indicado ([NV87]) uma solução negativa consistente para o Problema de Scarborough e Stone, merecendo destaque o fato de que, enquanto que nas soluções negativas consistentes obtidas anteriormente os modelos usados sempre eram tais que valia a igualdade " $\mathfrak{b}=\mathfrak{c}$ ", no modelo de Balcar, Frankiewicz e Mills vale "b $<\mathfrak{c} "$.

Vejamos outra aplicação dos FR-espaços como uma boa fonte de exemplos. Para isso, trataremos um pouco dos chamados espaços absolutamente enumeravelmente compactos. Essa classe de espaços topológicos foi introduzida por Matveev ([Mat94]) tendo como motivação o seguinte fato: sabe-se ([Eng89], 3.12.23(d) ) que um espaço de Hausdorff $X$ é enumeravelmente compacto se e só se para toda cobertura aberta $\mathcal{U}$ de $X$ (enumerável ou não) existe $F \subset X$ finito tal que $S t(F, \mathcal{U})=\bigcup\{U \in \mathcal{U}: U \cap F \neq \emptyset\}=X$.

\section{Definição 4.5 Um espaço topológico $X$ é dito absolutamente enumeravelmente com-} pacto (a.e.c.) se para toda cobertura aberta $\mathcal{U}$ de $X$ e para todo $Y \subseteq X$ denso existe $F \subset Y$ finito tal que $S t(F, \mathcal{U})=X$.

Obviamente, espaços $T_{2}$ a.e.c. são enumeravelmente compactos. Observamos agora que, se $X$ é separável e $D \subseteq X$ é enumerável e denso, então, dada $\mathcal{U}$ cobertura aberta de $X,\{S t(x, \mathcal{U}): x \in D\}$ é cobertura aberta enumerável de $X$; nessas condições, se $X$ é enumeravelmente compacto, existe $F \subset D$ finito para o qual $S t(F, \mathcal{U})=X$. Assim, se $X$ é enumeravelmente compacto e é hereditariamente separável (mais geralmente, se possui tightness enumerável ([Mat94],1.8) ), tem-se que $X$ é a.e.c. É natural, portanto, a seguinte questão de Matveev (1.11): existe um espaço topológico $X$ que seja enumeravelmente compacto, separável e não a.e.c. ? 
Vaughan ([Vau95]) apresentou uma resposta afirmativa a essa questão utilizando FR-espaços. A construção desse exemplo depende apenas de resultados que já fizemos em nosso trabalho, mais o clássico resultado de Hewitt-Marczewski-Pondiczery que, em sua versão enumerável, garante que o produto de no máximo c espaços separáveis é separável, e mais o seguinte

Teorema 4.11 Se $X$ é um espaço que possui pelo menos um subespaço não-vazio, fechado e compacto $K$, e $\mathcal{U}$ é uma cobertura aberta de $X$ que não possui subcobertura finita, então o espaço produto $X^{\kappa}$, onde $\kappa=|\mathcal{U}|$, não é a.e.c.

Demonstração Sejam $X$ e $\mathcal{U}$ como no enunciado; assumiremos que $|\mathcal{U}|=\kappa$ é a menor cardinalidade de uma cobertura aberta que não possua subcobertura finita. É suficiente mostrarmos que $X^{\kappa}$ não é a.e.c., pois a propriedade "ser a.e.c." é preservada por aplicações contínuas abertas sobrejetoras ([M94],3.2), logo " $X^{\kappa}$ não é a.e.c." implica " $X^{\mu}$ não é a.e.c. para todo $\mu \geqslant \kappa "$.

Por hipótese, existe $\emptyset \neq K \subset X$, compacto e fechado, portanto existe $\mathcal{U}_{0} \subset \mathcal{U}$ finito que cobre $K$; definimos então

$$
\mathcal{U}^{\prime}=\mathcal{U}_{0} \cup\left\{U \backslash K^{\prime}: U \in \mathcal{U} \backslash \mathcal{U}_{0}\right\}
$$

Um rápido "estudo de casos" nos mostra que $\mathcal{U}^{\prime}$ é cobertura aberta de $X$, e é imediato que $\mathcal{U}^{\prime}$ não possui subcobertura finita. Segue que $\left|\mathcal{U}^{\prime}\right|=\kappa$ e que os únicos abertos de $\mathcal{U}^{\prime}$ que interceptam $K$ são os elementos de $\mathcal{U}_{0}$. Assumiremos portanto s.p.g que a cobertura original $\mathcal{U}$ possui essas propriedades e que $\mathcal{U}=\left\{U_{\alpha}: \alpha<\kappa\right\}$, sendo que $\mathcal{U}_{0}=\left\{U_{0}, U_{1}, \ldots, U_{n}\right\}$ para algum $n<\omega$.

Considere agora o produto $X^{\kappa}$ e a família $\mathcal{W}$ de abertos básicos na topologia produto $\mathcal{W}=\left\{\prod_{0}^{-1}\left[U_{\alpha}\right] \cap \prod_{\alpha}^{-1}\left[U_{\beta}\right]: \alpha, \beta<\kappa\right\}$. Afirmamos que $\mathcal{W}$ cobre $X^{\kappa}$. De fato: seja $f \in{ }^{\kappa} X$. Entao existe $\alpha_{0}<\kappa$ tal que $f(0) \in U_{\alpha_{0}}$; daí existe $\beta_{0}<\kappa$ para o qual $f\left(\alpha_{0}\right) \in U_{\beta_{0}}, \operatorname{logo} f \in \prod_{0}^{-1}\left[U_{\alpha_{0}}\right] \cap \prod_{\alpha_{0}}^{-1}\left[U_{\beta_{O}}\right]$. Definimos agora um subconjunto $Y \subset X^{\kappa}$ pondo

$$
Y=\left\{f \in{ }^{\kappa} X:\{\alpha<\kappa: f(\alpha) \notin K\} \text { é finito }\right\}
$$

e observamos que $Y$ é denso em $X^{\kappa}$, pois, se $V$ é um aberto básico de $X^{\kappa}$, então $V$ é da forma $V=\prod_{\alpha<\kappa} X_{\alpha} \operatorname{com}\left\{\xi<\kappa: X_{\xi} \neq X\right\}$ finito e é fácil construir funções $f \in{ }^{\kappa} X$ tais que 
$f(\alpha) \in K$ para todo $\alpha \in \kappa \backslash\left\{\xi<\kappa: X_{\xi} \neq X\right)$; tais $f$ pertencem a $V \cap Y$. Mostraremos agora que $X^{\kappa}$ não é a.e.c. verificando que, se $F \subset Y$ é finito, então

$$
\bigcup\{W \in \mathcal{W}: W \cap F \neq \emptyset\}=S t(F, \mathcal{W}) \neq X^{\kappa}
$$

De fato: se $f \in F, R_{f}=\{\alpha<\kappa: f(\alpha) \notin K\}$ é finito logo $R=\bigcup\left\{R_{f}: f \in F\right\}$ é finito e dessa forma existe $p \in X \backslash \bigcup\left\{U_{\alpha}: \alpha \in R \cup\{0,1, \ldots, n\}\right\}$. Considere $g \in{ }^{\kappa} X$ a função que tem valor constante $p$; afirmamos que $g \notin S t(F, \mathcal{W})$. De fato: suponha por absurdo que existam $W \in \mathcal{W}$ e $f \in F$ tais que $f, g \in W$. Então existem $\alpha, \beta<\kappa$ tais que $W=\prod_{0}^{-1}\left[U_{\alpha}\right] \cap \prod_{\alpha}^{-1}\left[U_{\beta}\right]$; logo, como $g(0)=g(\alpha)=p$, temos $p \in U_{\alpha} \cap U_{\beta}$ e portanto $\alpha, \beta \notin R \cup\{0,1, \ldots, n\}$. De $\alpha \notin R$ temos $f(\alpha) \in K$. Mas, os únicos elementos de $\mathcal{U}$ que interceptam $K$ são os elementos de $\mathcal{U}_{0}$; como $f(\alpha) \in U_{\beta} \cap K$, temos $U_{\beta} \cap K \neq \emptyset \mathrm{e}$ $\beta \in\{0,1, \ldots, n\}$, o que é uma contradição.

Corolário Se $X$ é um espaço $T_{1}$ não-compacto, então existe um cardinal infinito $\kappa$ tal que $X^{\kappa}$ não é a.e.c.

Tendo o Teorema, segue imediatamente o

Exemplo 4.3 Existe um espaço topológico enumeravelmente compacto e separável que não é absolutamente enumeravelmente compacto.

Prova: Considere $\mathcal{T}$ uma torre com $|\mathcal{T}|=\mathfrak{t}$ e $X=X(\mathcal{T})$ o FR-espaço seqüencialmente compacto não-compacto associado. Então $X$ é separável, os abertos básicos de $X$ são fechados, compactos não-vazios e $X$ possui uma cobertura aberta $\mathcal{U}$ tal que $|\mathcal{U}|=\mathfrak{t}$ e $\mathcal{U}$ não possui subcobertura finita. Então $X^{t}$ é um espaço separável (por Hewitt-MarczewskiPondiczery, já que $\mathfrak{t} \leqslant \mathfrak{c}$ ), enumeravelmente compacto (pelo Teo. 4.5) e não a.e.c., pelo Teorema anterior.

\subsection{O cardinal $\mathfrak{d}$ e a cobertura dos irracionais por compactos}


Conforme destacamos em nossas preliminares, o espaço $\mathbb{P}$ dos irracionais, com a topologia de subespaço da reta, é homeomorfo ao espaço produto $\omega^{\omega}$. Além disso, como $\mathbb{P}$ é um subconjunto $G_{\delta}$ do espaço métrico completo $\mathbb{R}\left(\mathbb{P}=\bigcap_{r \in \mathbb{Q}} \mathbb{R} \backslash\{r\}\right)$, temos que $\mathbb{P}$ é completamente metrizável ([Wil70], 24.12), isto é, existe uma métrica sobre $\mathbb{P}$ que é equivalente à métrica usual (logo, dá a $\mathbb{P}$ a topologia de subespaço de $\mathbb{R}$ ) mas que torna $\mathbb{I P}$ um espaço métrico completo. Sabemos que, na métrica usual, $\mathbb{P}$ não é completo; este é mais um exemplo do fato de que a completude é uma propriedade métrica e não topológica.

De $\mathbb{P}$ completamente metrizável, segue que seus subconjuntos compactos são fechados (e limitados) em $\mathbb{P}$, e é fácil ver que um compacto $C \subset \mathbb{P}$ deve ter interior vazio em $\mathbb{P}$ (caso contrário é fácil exibir subconjuntos infinitos de $C$ sem pontos de acumulação). $\mathrm{Na}$ verdade, vale o seguinte fato, bastante conhecido e que foi destacado por Hechler em [Hec75a]:

Fato 4.5 Um subconjunto $C \subset \mathbb{P}$ é compacto em $\mathbb{P}$ se e só se $C$ é um subconjunto fechado, limitado e de interior vazio em $\mathbb{P}$ que também é fechado como subconjunto de IR.

Considere então o cardinal $\kappa$ dado por

$\kappa=\min \{\lambda$ : existe uma cobertura $\mathcal{U}$ de $\mathbb{P}$ formada por subconjuntos compactos de $\mathbb{P} \operatorname{com}|\mathcal{U}|=\lambda\}$

A cobertura $\{\{x\}: x \in \mathbb{P}\}$ é formada por $\mathfrak{c}$ compactos (unitários), logo $\kappa \leqslant$ c. Como $\mathbb{P}$ é completamente metrizável, $\mathbb{P}$ é um espaço de Baire e qualquer união enumerável de fechados com interior vazio em $\mathbb{P}$ possui interior vazio em $\mathbb{P}$, donde $\mathbb{P}$ não pode ser coberto por uma família enumerável de subconjuntos compactos. Assim, $\omega_{1} \leqslant \kappa \leqslant \mathfrak{c}$. No entanto, podemos dar uma resposta mais precisa, usando um dos nossos pequenos cardinais. Apenas com o intuito de formalizar um pouco mais nossa discussão, considere a função cardinal compact covering number, denotada por $k c$, que é definida de modo que, se $X$ é um espaço topológico, $k c(X)$ é a cardinalidade mínima de uma cobertura de $X$ por subconjuntos compactos de $X$. Vale então o seguinte teorema, devido a Hechler ([Hec75a]): 
Teorema $4.12 k c(\mathbb{P})=\mathfrak{d}$, isto é, o espaço $\mathbb{P}$ dos irracionais pode ser coberto por $\mathfrak{o}$ subconjuntos compactos de $\mathbb{P}$, e não por menos que d subconjuntos compactos.

Antes de demonstrarmos o Teorema, fixemos algumas notações: $\prod_{n}:{ }^{\omega} \omega \mapsto \omega$ é a usual projeção na n-ésima coordenada (i.e., se $f \in{ }^{\omega} \omega$ tem-se $\prod_{n}(f)=f(n)$ ) e, dado $f \in$ ${ }^{\omega} \omega$, denotaremos por $C_{f}$ o conjunto $C_{f}=\left\{g \in \omega_{\omega}: g \leqslant f\right\}$ (relembrando que " $g \leqslant f$ " significa " $g(n) \leqslant f(n)$ para todo $n<\omega$ "). Note que para qualquer $f \in{ }^{\omega} \omega$ temos $C_{f} \subseteq$ ${ }^{\omega} \omega$ compacto em $\omega^{\omega}$, pois $C_{f}=\prod_{i<\omega}[0, f(i)]$.

Demonstração Utilizaremos a identificação entre $\mathbb{P}$ e $\omega^{\omega}$ e a igualdade "do $=\mathfrak{d}_{1}$ " do Teo. $2.3\left(\mathfrak{d}_{1}=\min \left\{|D|: D\right.\right.$ é cofinal em $\left.\left.\left\langle{ }^{\omega} \omega, \leqslant\right\rangle\right\}\right)$. Basta portanto mostrar que $k c\left(\omega^{\omega}\right)=\mathfrak{d}_{1}$.

Prova de $\mathfrak{d}_{1} \leqslant k c\left(\omega^{\omega}\right)$ : Seja $\mathcal{U}$ uma cobertura de $\omega^{\omega}$ por compactos com $|\mathcal{U}|=k c\left(\omega^{\omega}\right)$. Dados $C \in \mathcal{U}$ e $n<\omega$ temos $\prod_{n}[C]$ compacto em $\omega$, portanto finito, donde podemos definir para cada $C \in \mathcal{U}$ uma função $f_{C} \in{ }^{\omega} \omega$ pondo $f_{C}(n)=\max \left(\prod_{n}[C]\right)$ para todo $n<\omega$. Afirmamos que $\left\{f_{C}: C \in \mathcal{U}\right\}$ é cofinal em $\left\langle{ }^{\omega} \omega, \leqslant\right\rangle$, donde segue a desigualdade. Seja então $g \in{ }^{\omega} \omega$ qualquer; como $\mathcal{U}$ é cobertura de $\omega^{\omega}$ existe $C \in \mathcal{U}$ tal que $g \in C$. Daí segue que

$$
(\forall n<\omega)\left[g(n) \leqslant \max \left(\prod_{n}[C]\right)=f_{C}(n)\right]
$$

donde $g \leqslant f_{C}$ e $\left\{f_{C}: C \in \mathcal{U}\right\}$ é cofinal em $\left\langle{ }^{\omega} \omega, \leqslant\right\rangle$, conforme desejado.

Prova de $k c\left(\omega^{\omega}\right) \leqslant \mathfrak{d}_{1}$ : Seja $D \subseteq{ }^{\omega} \omega$ cofinal em $\left\langle{ }^{\omega} \omega, \leqslant\right\rangle$, com $|D|=\mathfrak{o}_{1}$; afirmamos que $\left\{C_{f}: f \in D\right\}$ é uma cobertura de $\omega^{\omega}$, e como cada $C_{f}$ é compacto teremos obtido o desejado. De fato: se $g \in{ }^{\omega} \omega$, como $D$ é cofinal em $\left\langle{ }^{\omega} \omega, \leqslant\right\rangle$ existe $f \in D$ para a qual $g \leqslant f$ e portanto $g \in C_{f}$. Assim, $\left\{C_{f}: f \in D\right\}$ cobre $\omega^{\omega}$.

Observamos que, se $C \subset \mathbb{P}\left(\equiv \omega^{\omega}\right)$ é compacto, então existe $f \in{ }^{\omega} \omega$ tal que $C \subseteq C_{f}$; mais especificamente, para todo $C \subset \mathbb{P}$ compacto vale que $C \subseteq C_{f_{C}}$. Vale também destacar que, se $f \in{ }^{\omega} \omega$ é uma função que assume infinitas vezes valores não nulos, então $\left|C_{f}\right|=\mathfrak{c}$. 


\subsection{A normalidade do produto de um espaço de Lindelöf com os irracionais e o cardinal $\mathfrak{b}$}

Em 1963, Michael ([Mic63]) mostrou que o produto de um espaço normal com um espaço métrico não é necessariamente normal. O exemplo de Michael surgiu como parte do esforço em se resolver uma "velha questão", ainda em aberto na época, que era encontrar em ZFC um exemplo do que hoje denominados de espaço de Dowker - um espaço normal cujo produto com o intervalo $[0,1]$ não é normal; tal questão foi resolvida por Mary Ellen Rudin - com um exemplo "ZFC puro" - em 1971 (existe um artigo de M.E.Rudin sobre tais espaços no Handbook.).

No exemplo de Michael, o espaço normal é a chamada reta de Michael $M$ que é o refinamento da topologia de $\mathbb{R}$ obtida isolando-se os irracionais, isto é, $M$ é o espaço topológico com suporte na reta e gerado pela base $\{U \subseteq M: U$ é aberto em $\mathbb{R}$ \}$\cup\{\{x\}: x \in \mathbb{P}\}$ - e o èspaço métrico é o espaço dos irracionais $\mathbb{P} ; M$ é hereditariamente paracompacto ([Eng89], 5.1.32) e $\mathbb{P}$ é separável. No entanto, $M$ não é nem Lindelöf nem separável. Em seu artigo de 1963, Michael destaca que, sob $C H$, é possível construir um espaço de Lindelöf hereditariamente paracompacto cujo produto com os irracionais não é normal (Footnote 4); tal espaço seria um subespaço não-enumerável de $M$ concentrado em $Q$ - definiremos em seguida o conceito de "concentração". Veremos também que a hipótese "CH" pode ser enfraquecida para "bo $=\omega_{1}$ ".

Definição 4.6 Sejam $X$ um espaço topológico e $A \subseteq X$. Dizemos que $X$ é concentrado em $A$ se para todo aberto $U$ que contém $A, X \backslash U$ é (no máximo) enumerável.

A partir deste momento e até o final desta seção, a palavra "espaço" será sempre uma abreviação de "espaço regular". Portanto, quando nos referirmos a um "espaço de Lindelöf", tal espaço será normal e paracompacto ([Eng89], 3.8.2 e 3.8.11.).

Nossa primeira meta para esta seção é demonstrar o

Teorema 4.13 Se $\mathfrak{b}=\omega_{1}$, existe um espaço de Lindelöf cujo produto com os irracionais não é normal. 
Para isso, vamos inicialmente dar uma caracterização dos subespaços compactos e $\sigma$-compactos de $\mathbb{P}$ tendo como ponto de partida sua identificação com o espaço-produto $\omega^{\omega}$. Dada $f \in{ }^{\omega} \omega$, definimos $C_{f}$ como na seção anterior, i.e., $C_{f}=\left\{g \in{ }^{\omega} \omega: g \leqslant f\right\}$; já vimos que cada $C_{f}$ é compacto e que, se $C \subset \omega^{\omega}$ é compacto, então existe $f \in{ }^{\omega} \omega$ tal que $C \subseteq C_{f}$. Definimos agora, para cada $f \in{ }^{\omega} \omega, C_{f}^{*}=\left\{g \in{ }^{\omega} \omega: g \leqslant{ }^{*} f\right\}$ e afirmamos que, dada $f \in{ }^{\omega} \omega, C_{f}^{*}$ é $\sigma$-compacto: de fato, para cada $i, j<\omega$ definimos $f_{i, j} \in{ }^{\omega} \omega$ pondo, para cada $n<\omega$,

$$
f_{i, j}(n)= \begin{cases}i & \text { se } n \leqslant j \\ f(n) & \text { caso contrário }\end{cases}
$$

e é fácil ver que $C_{f}^{*}=\bigcup_{i, j<\omega} C_{f_{i, j}}$. Mais ainda: podemos afirmar que, se $S \subset \omega^{\omega}$ é $\sigma$ compacto, então existe $f \in{ }^{\omega} \omega$ tal que $S \subseteq C_{f}^{*}$; basta notar que, se $S \subset \omega^{\omega}$ é $\sigma$-compacto, então existe uma subfamília $\left\{g_{k}: k<\omega\right\} \subseteq{ }^{\omega} \omega$ tal que $S \subseteq \bigcup_{k<\omega} C_{g_{k}}$, e, definindo-se $h(n)=\sum_{k \leqslant n} g_{k}(n)$ tem-se $\bigcup_{k<\omega} C_{g_{k}} \subseteq C_{h}^{*}$.

É fácil ver também que, dadas $f, g \in{ }^{\omega} \omega, C_{f} \subseteq C_{g}$ (respectivamente, $C_{f}^{*} \subseteq C_{g}^{*}$ ) se e só se $f \leqslant g$ (respectivamente, $f \leqslant{ }^{*} g$ ).

Podemos agora enunciar a

Proposição 4.1 Seja $X$ um subconjunto não-enumerável de $\mathbb{P}\left(\equiv \omega^{\omega}\right)$. São equivalentes as seguintes afirmações sobre $X$ :

(i) Todo subconjunto não-enumerável de $X$ é ilimitado em $\left\langle{ }^{\omega} \omega, \leqslant^{*}\right\rangle$.

(ii) $X$ tem interseç̧ão enumerável com qualquer subconjunto compacto de $\mathbb{P}$.

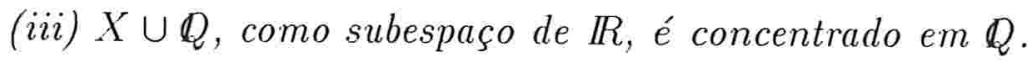

(iv) $X \cup Q$, como subespaço de $\mathbb{M}$, é um espaço de Lindelöf.

Demonstração Seja (*) a asserção

(*) $X$ é um subconjunto não-enumerável de $\mathbb{P}\left(\equiv \omega^{\omega}\right)$ que possui intersecção enumerável com $C_{g}^{*}$ para todo $g \in{ }^{\omega_{\omega}}$.

Inicialmente mostraremos que $(i)$ e $(i i)$ são equivalentes a $(*)$. Note que se $A$ é um subconjunto de ${ }^{\omega} \omega$, então uma função $f \in{ }^{\omega} \omega$ é limitante de $A$ em $\left\langle{ }^{\omega} \omega, \leqslant^{*}\right\rangle$ se e só se 
$A \subseteq C_{f}^{*}$

Prova de $(i) \Rightarrow(*)$ : Se $X$ possuísse intersecção não-enumerável com $C_{g}^{*}$ para algum $g \in$ ${ }^{\omega} \omega, C_{g}^{*} \cap X$ seria um subconjunto não-enumerável e limitado de $X$.

Prova de $(*) \Rightarrow(i)$ : Se $A \subseteq X$ fosse não-enumerável e limitado, existiria $f \in{ }^{\omega} \omega$ tal que $A \subseteq C_{f}^{*}$ e então $X \cap C_{f}^{*}$ seria não-enumerável.

Prova de $($ ii $) \Leftrightarrow(*)$ : Reunião enumerável de enumeráveis é enumerável.

Para a equivalência entre (ii) e (iii), utilizaremos a caracterização dos compactos de $\mathbb{P}$ dada pelo Fato 4.5 da seção anterior e o seguinte resultado de Topologia, de verificação imediata: "Se $X$ é um espaço topológico, $F$ é um subconjunto fechado de $X$ e $D$ é um subconjunto denso de $X$, então $F$ tem interior vazio em $X$ se e só se $F \cap D$ tem interior vazio em $D^{\prime \prime}$.

Prova de (ii) $\Rightarrow$ (iii): Seja $U \subseteq X \cup \mathbb{Q}$ um aberto qualquer de $X \cup \mathbb{Q}$ que contenha $\mathbb{Q}$; então existe $U^{\prime}$, aberto da reta, tal que $\mathbb{Q} \subset U^{\prime}, U=U^{\prime} \cap(X \cup Q)$ e $(X \cup Q) \backslash U=X \backslash U^{\prime}$, e portanto $X \backslash U^{\prime}$ é o complementar de $U$ em $X \cup \mathbb{Q}$, está contido em (no suporte de) $\mathbb{P}$ e é tal que $X \backslash U^{\prime} \subseteq \mathbb{R} \backslash U^{\prime}$, onde $\mathbb{R} \backslash U^{\prime}$ é um fechado da reta contido em $\mathbb{P}$ e portanto $\sigma$-compacto em $\mathbb{I P}$; segue que $X \backslash U^{\prime}$ é enumerável, caso contrário possuiria intersecção não-enumerável com algum dos enumeráveis compactos (de $\mathbb{P}$ ) cuja união é $\mathbb{R} \backslash U^{\prime}$. Como $U$ era um aberto arbitrário, $X \cup Q$ é concentrado em $Q$.

Prova de (iii) $\Rightarrow($ ii $)$ : Assumindo (iii), suponhamos por absurdo que exista $C \subset \mathbb{P}$ compacto em $\mathbb{P}$ para o qual $X \cap C$ seja não-enumerável. Segue que $\mathbb{R} \backslash C$ é um aberto da reta que contém $\mathbb{Q}$ e portanto $U=(\mathbb{R} \backslash C) \cap(X \cup \mathbb{Q})$ é um aberto de $X \cup \mathbb{Q}$ que contém $\mathbb{Q}$ e tal que $(X \cup Q) \backslash U=X \cap C$ é não-enumerável, o que contradiz a hipótese de $X \cup Q$ ser concentrado em $Q$; logo, $X$ deve possuir intersecção enumerável com qualquer compacto de $\mathbb{P}$.

A equivalência entre (iii) e (iv) segue imediatamente das definições de "concen- 
trado", "Lindelöf" e da topologia de $M$.

O cardinal $\mathfrak{b}$ entra em nossa discussão agora com a seguinte

Proposição 4.2 Existe um subconjunto não-enumerável $X \subseteq \mathbb{P}$ satisfazendo cada uma das quatro condições dadas na Proposição anterior se e só se $\mathfrak{b}=\omega_{1}$.

Demonstração Se $X \subseteq \mathbb{P}$ é não-enumerável e satisfaz as condições da Proposição anterior, tomamos $Y \subseteq X \operatorname{com}|Y|=\omega_{1}$ e a condição $(i)$ nos dá $Y$ ilimitado em $\left\langle{ }^{\omega} \omega, \leqslant^{*}\right\rangle$, donde $\mathfrak{b}=\omega_{1}$. Por outro lado, se $\mathfrak{b}=\omega_{1}$, usamos a igualdade $\mathfrak{b}=\mathfrak{b}_{1}$ do Teo.2.2 e tomamos $X=\left\{f_{\alpha}: \alpha<\omega_{1}\right\}$ ilimitado em $\left\langle{ }^{\omega} \omega, \leqslant^{*}\right\rangle$, bem-ordenado por $<^{*}$ e enumerado pelo seu tipo de ordem; $X$ claramente satisfaz a condição $(i)$ da Proposição anterior.

Agora estamos em condições de demonstrar o Teorema 4.13.

Demonstração do Teorema 4.13: Assumindo $b=\omega_{1}$, existe $X \subset \mathbb{P}$ não-enumerável e tal que $Y=X \cup Q$, com a topologia de subespaço de $M$, é um espaço de Lindelöf. Afirmamos que $Y \times \mathbb{P}$ não é normal. De fato: um resultado de M.E.Rudin e Starbird de 1975 nos garante que o produto de um espaço de Lindelöf com um espaço métrico separável é normal se e só se é Lindelöf, conforme destacou Lawrence em [Law90], Lema 1. Mas, $Y \times \mathbb{P}$ não é Lindelöf, pois $\{\langle x, x\rangle: x \in X\}$ é um subespaço fechado, discreto e não-enumerável de $Y \times \mathbb{P}$.

Destacamos o fato de que o espaço de Lindelöf $Y$ utilizado no teorema anterior foi obtido a partir da técnica de tomar-se um espaço métrico separável e refinar sua topologia isolando determinados pontos; a existência de um subespaço do refinamento concentrado no conjunto derivado e tal que seu produto com $\mathbb{P}$ é não-normal foi garantida pela condição "b $=\omega_{1}$ ". Note também que, na construção feita, $\mathbb{Q}$, como conjunto derivado do refinamento, é um subespaço fechado de $Y=X \cup \emptyset$ e que $\mathbb{Q}$, como subespaço de $Y$, de $M$ ou de $\mathbb{R}$, consiste no mesmo espaço topológico (metrizável), donde $\boldsymbol{Q} \times \mathbb{I}$ é normal; e é fácil ver que o mesmo aconteceria com o "conjunto derivado do refinamento" em qualquer processo baseado no método de isolar pontos num espaço métrico separável.

A discussão que fizemos no parágrafo anterior tem por objetivo justificar a definição de espaço de Michael concentrado. 
Definição 4.7 (i) Um espaço $X$ é dito um espaço de Michael se $X$ é um espaço de Lindelöf cujo produto com $\mathbb{P}$ é não-normal.

(ii) Um espaço de Michael X é dito um espaço de Michael concentrado se $X$ for concentrado num subconjunto $A \subset X$ fechado e tal que $A \times \mathbb{P}$ é normal.

Assim, o conceito de "espaço de Michael concentrado" é definido de tal forma a englobar qualquer caso construído a partir do método de isolar pontos num espaço métrico separável (sendo, neste caso, " $A=$ o conjunto derivado do refinamento").

O espaço $Y=X \cup Q$ construído na demonstração do Teo.4.13 nos mostra, portanto, que "b $=\omega_{1}$ " implica na existência de um espaço de Michael concentrado. No entanto, podemos afirmar mais. De certa forma, " $\mathfrak{b}=\omega_{1}$ " é a única chance do método de isolar pontos num métrico separável obter êxito. O seguinte resultado é devido a Lawrence ([Law90]).

Teorema 4.14 (Lawrence) Existe um espaço de Michael concentrado se e só se $\mathfrak{b}=\omega_{1}$.

A demonstração que apresentaremos para esse Teorema é devida a Peg Daniels ([Dan95]). Daniels, em sua demonstração, utilizou o conceito (por ela definido) de $\omega^{\omega}$ direcionamento.

Definição 4.8 Uma propriedade $P$ é dita $\omega^{\omega}$-direcionada se um espaço $X$ necessariamente possui a propriedade $P$ sempre que $X$ puder ser escrito na forma $X=\bigcup_{f \in \omega^{\omega}} X_{f}$, onde cada $X_{f}$ é um subespaço fechado de $X$ que satisfaz $P$ e $f \leqslant g$ implica $X_{f} \subseteq \stackrel{f \in \omega^{\omega}}{X_{g}}$ para quaisquer $f, g \in{ }^{\omega} \omega$.

A seguinte proposição tem papel central na demonstração do Teo.4.14.

Proposição 4.3 (Daniels) A propriedade "ser enumerável" é $\omega^{\omega}$-direcionada se e só se $\mathfrak{b}>\omega_{1}$.

Demonstração Inicialmente mostraremos que, se toda subfamília de ${ }^{\omega} \omega$ de cardinalidade $\omega_{1}$ for limitada em $\left\langle{ }^{\omega} \omega, \leqslant^{*}\right\rangle$ (isto é, se $\mathfrak{b}>\omega_{1}$ ) então "ser enumerável" é $\omega^{\omega}$-direcionada. Seja então $X$ um espaço tal que $X=\bigcup_{f \in \omega^{\omega}} X_{f}$, sendo $\left\{X_{f}: f \in{ }^{\omega} \omega\right\}$ uma família de fechados enumeráveis de $X$ que satisfazem " $f \leqslant g \Rightarrow X_{f} \subseteq X_{g}$ ". Suponhamos por absurdo que $X$ não seja enumerável; sejam então $\left\{x_{\alpha}: \alpha<\omega_{1}\right\} \subseteq X$ e $\left\langle f_{\alpha}: \alpha<\omega_{1}\right\rangle$ 
uma seqüência de funções de ${ }^{\omega} \omega$ tais que $x_{\alpha} \in X_{f_{\alpha}}$ para todo $\alpha<\omega_{1}$. Como cada um dos fechados $X_{f}$ é enumerável, podemos supor que a aplicação $\alpha \mapsto f_{\alpha}$ é injetora e assim assumiremos que $\operatorname{im}\left(\left\langle f_{\alpha}: \alpha<\omega_{1}\right\rangle\right)=\left\{f_{\alpha}: \alpha<\omega_{1}\right\}$. Da hipótese segue agora que existe $f \in{ }^{\omega} \omega$ tal que $\left(\forall \alpha<\omega_{1}\right)\left[f_{\alpha} \leqslant{ }^{*} f\right]$ e é fácil construir para cada $\alpha<\omega_{1}$ uma função $g_{\alpha} \in{ }^{\omega} \omega$ que satisfaça $f_{\alpha} \leqslant g_{\alpha}$ e $g_{\alpha}={ }^{*} f$. Mas, a classe de equivalência de $f$ por $={ }^{*}$ é enumerável; segue que existem $S \subseteq \omega_{1}$ não-enumerável e $g \in{ }^{\omega} \omega$ tais que $(\forall \alpha \in S)\left[f_{\alpha} \leqslant g\right]$, donde $\left\{x_{\alpha}: \alpha \in S\right\} \subseteq \bigcup_{\alpha \in S} X_{f_{\alpha}} \subseteq X_{g}$, o que é uma contradição com $\left|X_{g}\right| \leqslant \omega$. Logo, $X$ é enumerável, portanto sob $b>\omega_{1}$ "ser enumerável" é $\omega^{\omega}$-direcionada.

Para a recíproca, assumiremos que $\mathfrak{b}=\omega_{1}$ e construiremos um espaço $X$ que sirva de contra-exemplo. Usando a igualdade $\mathfrak{b}=\mathfrak{b}_{1}$ do Teo.2.2, consideremos $X=\left\{g_{\alpha}: \alpha<\right.$ $\left.\omega_{1}\right\}$ uma subfamília de ${ }^{\omega} \omega$ ilimitada em $\left\langle{ }^{\omega} \omega, \leqslant^{*}\right\rangle$, bem-ordenada por $<^{*}$ e enumerada pelo seu tipo de ordem, com a topologia de subespaço de $\omega^{\omega}$. Para cada $f \in{ }^{\omega} \omega$, consideramos $X_{f}=\{g \in X: g \leqslant f\}=X \cap C_{f}$; então $X=\bigcup_{f \in \omega^{\omega}} X_{f}$ e cada $X_{f}$ é enumerável (já que subconjuntos não-enumeráveis de $X$ são ilimitados). Resta estabelecer que cada $X_{f}$ é fechado em $X$ e $X$ será ùm contra-exemplo para o $\omega^{\omega}$-direcionamento de "ser enumerável". Mas, isso é imediato, pois os $C_{f}$ são compactos no espaço de Hausdorff $\omega_{\omega} \omega$ e são portanto fechados; como $X_{f}=X \cap C_{f}$ para toda $f$ o resultado segue.

Demonstração do Teorema 4.14: "b $=\omega_{1}$ " implica na existência de um espaço de Michael concentrado, conforme já destacamos. Mostraremos a recíproca pela contrapositiva, verificando que, se $\mathfrak{b}>\omega_{1}$, não existem espaços de Michael concentrados.

Assumamos então $\mathfrak{b}>\omega_{1}$. Suponha que $X$ seja um espaço de Lindelöf concentrado num subconjunto $A \subset X$ fechado tal que $A \times \omega^{\omega}$ é normal (ou, equivalentemente, Lindelöf, como já observamos). Mostraremos que $X \times \omega^{\omega}$ é Lindelöf, o que nos garantirá que $X$ não é um espaço de Michael.

Seja $\mathcal{U}$ uma cobertura aberta qualquer de $X \times \omega^{\omega}$ e $\mathcal{V} \subset \mathcal{U}$ enumerável e tal que $\bigcup \mathcal{V}$ cobre $A \times \omega^{\omega}$. Seja $H=\left(X \times \omega^{\omega}\right) \backslash \bigcup \mathcal{V}$ e sejam $\prod_{1}$ e $\prod_{2}$ as projeções do produto em $X$ e $\omega^{\omega}$ respectivamente. Se mostrarmos que $\prod_{2}[H]$ é enumerável, garantiremos que $H$ é uma união enumerável de espaços de Lindelöf $\left(H=\bigcup_{i \in \prod_{2}[H]} H \cap(X \times\{i\})\right)$ e, dessa forma, teremos que $H$ também é um espaço de Lindelöf, existindo portanto uma subfamília enumerável de $\mathcal{U}$ que cobre $H$. Dessa forma, estará garantida a existência de uma subcobertura enumerável de $\mathcal{U}$ e a demonstração estará encerrada. 
Definindo-se $H_{f}=\left\{g \in \prod_{2}[H]: g \leqslant f\right\}=\prod_{2}[H] \cap C_{f}$ para cada $f \in{ }^{\omega} \omega$, o mesmo argumento utilizado na prova da Proposição 4.3 nos dá $H_{f}$ fechado em $\prod_{2}[H]$ para toda $f$. Como $\prod_{2}[H]=\bigcup_{f \in \omega^{\omega}} H_{f}$, cada $H_{f}$ é fechado em $X$ e vale que " $f \leqslant g \Rightarrow H_{f} \subseteq H_{g}$ ", a hipótese "b $>\omega_{1}$ " nos dá que "ser enumerável" é $\omega^{\omega}$-direcionada (pela Prop.4.3) e portanto basta mostrar que cada $H_{f}$ é enumerável para concluirmos que $\prod_{2}[H]$ é enumerável.

Pois bem: para cada $i \in \prod_{2}[H]$, seja $x_{i} \in X \backslash A$ tal que $\left\langle x_{i}, i\right\rangle \in H$. Temos então $K=\left\{\left\langle x_{i}, i\right\rangle: i \in \prod_{2}[H]\right\} \subseteq H \subseteq\left(X \times \omega^{\omega}\right) \backslash\left(A \times \omega^{\omega}\right)$, donde $K \cap\left(A \times \omega^{\omega}\right)=\emptyset \mathrm{e}$, mais ainda, como $H$ é fechado, cada ponto de $\left(A \times \omega^{\omega}\right)$ possui uma vizinhança disjunta de $H$ e, portanto, de $K$. Suponha agora por absurdo que exista $g \in{ }^{\omega} \omega$ tal que $\left|H_{g}\right| \geqslant \omega_{1}$. Para cada $a \in A,\{a\} \times C_{g}$ é um compacto contido em $A \times \omega^{\omega}$, logo existe uma cobertura finita $\mathcal{W}_{a}$ de $\{a\} \times C_{g}$ formada por abertos básicos, $\mathcal{W}_{a}=\left\{U_{n, a} \times V_{n, a}: n \leqslant m_{a}\right\}$, sendo que cada um desses abertos básicos é disjunto de $K$. Agora, $\mathcal{W}=\left\{\bigcap_{n \leqslant m_{a}} U_{n, a}: a \in A\right\}$ é uma cobertura aberta de $A$; de $\left|H_{g}\right| \geqslant \omega_{1}$, existe um subconjunto $\left\{i_{\alpha}: \alpha<\omega_{1}\right\} \subseteq H_{g}$ e, como $\bigcup \mathcal{W}$ cobre todos a menos de no máximo enumeráveis elementos de $X$ (pois $X$ é concentrado em $\bar{A}$ ) existe $\alpha<\omega_{1}$ tal que $x_{i_{\alpha}} \in \bigcup \mathcal{W}$. Seja então $a_{\alpha} \in A$ tal que $x_{i_{\alpha}} \in \bigcap_{n \leqslant m_{a_{\alpha}}} U_{n, a_{\alpha}}$. Tem-se que $\left\langle a_{\alpha}, i_{\alpha}\right\rangle \in \bigcup \mathcal{W}_{a_{\alpha}}$, portanto existe $n \leqslant m_{a_{\alpha}}$ tal que $\left\langle a_{\alpha}, i_{\alpha}\right\rangle \in U_{n, a_{\alpha}} \times V_{n, a_{\alpha}}$. Daí, $\left\langle x_{i_{\alpha}}, i_{\alpha}\right\rangle \in\left(U_{n, a_{\alpha}} \times V_{n, a_{\alpha}}\right) \cap K$, o que é uma contradição. Logo, cada um dos $H_{g}$ é enumerável e mostramos o desejado. 


\subsection{Considerações finais}

Apesar de já bastante extenso, este trabalho não tem a menor intenção de declararse completo: temos ciência de que não abordamos vários tópicos, por exemplo quase todos os que constam nas seções 8, 9, 11, 12 e 13 de [vD84]. Dentre esses tópicos, gostaríamos de ter tido condições de tratar a respeito dos "espaços construídos a partir de famílias a.d." (seção 11 de [vD84]) e das relações existentes entre o cardinal b e alguns "enfraquecimentos da normalidade" (seção 12 de [vD84]). Este último tópico nos parece. particularmente interessante, pois a partir de considerações a respeito do cardinal $\mathfrak{b}$ e da chamada "propriedade wD" (ver [vD84], p.155, ou [Nyi90], p.131) vê-se que, assumindo "b $=\mathfrak{c} "$, algumas construções baseadas em técnicas de Ostaszewski e van Douwen podem ser levadas a cabo (ver o exemplo 13.1 de [vD84] e as considerações das páginas 131 a 137 de [Nyi90]).

Acreditamos, porém, que este texto cumpriu o papel anunciado em seu título: ser uma efetiva introdução aos pequenos cardinais e às suas aplicações em Topologia. Dessa forma, esperamos ter dado uma pequena mas bem-intencionada contribuição ao desenvolvimento da Topologia Conjuntística em nosso país. 


\section{Referências Bibliográficas}

[Boo76] D. Booth. A Boolean view of sequential compactness. Colloquium Mathematicum, 36(2):215-216, 1976.

[BS82] Aleksander Blaszczyk and Andrzej Szymański. Hausdorff's gaps versus normality. Bull. Acad. Polon. Sci. Sér. Sci. Math., 30(7-8):371-378, 1982.

[Dan95] Peg Daniels. $\omega^{\omega}$-directedness and a question of E. Michael. Comment. Math. Univ. Carolin., 36(1):115-121, 1995.

[Eng89] Ryszard Engelking. General Topology, volume 6 of Sigma Series in Pure Mathematics. Heldermann Verlag, Berlin, 1989.

[Fre84] D. H. Fremlin. Consequences of Martin's axiom, volume 84 of Cambridge Tracts in Mathematics. Cambridge University Press, Cambridge, 1984.

[Hau36] Felix Hausdorff. Summen von $\aleph_{1}$ mengen. Fundamenta Mathematicae, 26:241$255,1936$.

[Hec72] Stephen H. Hechler. Short complete nested sequences in $\beta N \backslash N$ and small maximal almost-disjoint families. General Topology and Appl., 2:139-149, 1972.

[Hec75a] Stephen H. Hechler. On a Ubiquitous Cardinal. Proceedings of American Mathematical Society, 52:348-352, 1975.

[Hec75b] Stephen H. Hechler. On some weakly compact spaces and their products. General Topol. Appl., 5:83-93, 1975.

[Hod84] R. Hodel. Cardinal functions. I. In Handbook of set-theoretic topology, pages 1-61. North-Holland, Amsterdam, 1984. 
[Jec78] Thomas Jech. Set theory. Academic Press, New York, 1978.

[JW97] Winfried Just and Martin Weese. Discovering modern set theory. II - Settheoretic tools for every mathematician, volume 18 of Graduate Studies in Mathematics. American Mathematical Society, Providence, RI, 1997.

[Kun80] Kenneth Kunen. Set Theory - an introduction to the independence proofs, volume 102 of Studies in Logic and the Foundations of Mathematics. North Holland Publishing Company, Amsterdam, 1980.

[Law90] L. Brian Lawrence. The influence of a small cardinal on the product of a Lindelö space and the irrationals. Proc. Amer. Math. Soc., 110(2):535-542, 1990.

[Mat94] Michael V. Matveev. Absolutely countably compact spaces. Topology Appl., 58(1):81-92, 1994.

[Mic63] E. Michael. The product of a normal space and a metric space need not be normal. Bull.Amer.Math.Society, 69:375-376, 1963.

[NV83] Peter J. Nyikos and Jerry E. Vaughan. On first countable, countably compact spaces. I. $\left(\omega_{1}, \omega_{1}^{*}\right)$-gaps. Trans. Amer. Math. Soc., 279(2):463-469, 1983.

[NV87] P. J. Nyikos and J. E. Vaughan. Sequentially compact, Franklin-Rajagopalan spaces. Proc. Amer. Math. Soc., 101(1):149-15.5, 1987.

[NV92] Peter J. Nyikos and Jerry E. Vaughan. The Scarborough-Stone problem for Hausdorff spaces. In Proceedings of the Symposium on General Topology and Applications (Oxford, 1989), volume 44, pages 309-316, 1992.

[Nyi90] Peter Nyikos. On first countable, countably compact spaces. III. The problem of obtaining separable noncompact examples. In Open problems in topology, pages 127-161. North-Holland, Amsterdam, 1990.

[Rot41] Fritz Rothberger. Sur les familles indenombrables de suites de nombres naturels et les problèmes concernant la propriété C. Proceedings of the Cambridge Philosophical Society, 37:109-126, 1941. 
[Rot48] Fritz Rothberger. On some problems of Hausdorff and of Sierpinski. Fundamenta Mathematicae, 35:29-46, 1948 .

[Rud56] W. Rudin. Homogeneity Problems in the Theory of Cěch Compactifications. Duke Mathematical Journal, 23:409-419, 1956.

[Sch93] Marion Scheepers. Gaps in $\omega^{\omega}$. In Set theory of the reals (Ramat Gan, 1991), volume 6 of Israel Math. Conf. Proc., pages 439-561. Bar-Ilan Univ., Ramat Gan, 1993.

[She84] Saharon Shelah. On cardinal invariants of the continuum. In Axiomatic set theory (Boulder, Colo., 1983), volume 31 of Contemp. Math., pages 183-207. Amer. Math. Soc., Providence, RI, 1984.

[Sol77] R. C. Solomon. Families of sets and functions. Cachoslovak Math. J., $27(102)(4): 556-5.59,1977$.

[SS66] C. T. Scarborough and A. H. Stone. Products of nearly compact spaces. Trans. Amer. Math. Soc., 124:131-147, 1966.

[TJ97] Artur Tomita and Lúcia Junqueira. Topologia geral - 1o.semestre de 1997. Notas de aula do IME/USP, 1997.

[Vau84] Jerry E. Vaughan. Countably compact and sequentially compact spaces. In Handbook of set-theoretic topology, pages 569-602. North-Holland, Amsterdam, 1984.

[Vau90] Jerry E. Vaughan. Small uncountable cardinals and topology. In Open problems in topology, pages 195-218. North-Holland, Amsterdam, 1990.

[Vau95] Jerry E. Vaughan. A countably compact, separable space which is not absolutely countably compact. Comment. Math. Univ. Carolin., 36(1):197-201, 1995.

[Vau96] Jerry E. Vaughan. Small cardinals. Topology Atlas Invited Contributions, 1996.

[vD76] Eric K. van Douwen. Hausdorff gaps and a nice countably paracompact nonnormal space. In Topology Proceedings, Vol. I (Conf., Auburn Univ., Auburn, Ala., 1976), pages 239-242. Math. Dept., Auburn Univ., Auburn, Ala., 1976. 
[vD84] Eric K. van Douwen. The integers and topology. In Handbook of set-theoretic topology, pages 111-167. North-Holland, Amsterdam, 1984.

[Wil70] Stephen Willard. General topology. Addison-Wesley Publishing Co., Reading, Mass.-London-Don Mills, Ont., 1970. 


\section{Índice Remissivo}

\begin{tabular}{|c|c|}
\hline$={ }^{*}, 5$ & $\mathfrak{a}, 1 \mathfrak{\imath}$ \\
\hline$A \subseteq \subseteq^{*} B, 5$ & $\mathfrak{b}, 1 \mathfrak{\imath}$ \\
\hline$A \backslash B, 1$ & $\mathfrak{c}, 3$ \\
\hline$A^{\prime}, 10$ & $\mathfrak{d}, 17$ \\
\hline$F \perp \mathcal{G}, 36$ & $\mathfrak{h}, 79$ \\
\hline$K_{X}, 37$ & m. 46 \\
\hline$L_{f}, 37$ & n, 76 \\
\hline$M A(r), 46$ & $\mathfrak{p}, 18$ \\
\hline$M A_{\sigma-c e n t r a d a s}, 47$ & $\mathfrak{s}, 18$ \\
\hline$T_{i}, 9$ & $\mathfrak{t}, 18$ \\
\hline$X(\mathcal{T}), 96$ & $\kappa^{+}, 3$ \\
\hline$X^{A}, 13$ & $\leqslant_{R K}, 14$ \\
\hline$[X]^{\lambda}, 3$ & $\omega^{\omega}, 13$ \\
\hline$[X]^{<\lambda}, 3$ & $\omega, 2$ \\
\hline$[\omega]^{\omega}, 3$ & $\omega^{\star} \cdot 1: 3$ \\
\hline$[\omega]^{<\omega}, 3$ & $\omega_{0}, 3$ \\
\hline$M, 105$ & $\omega_{\alpha}, 3$ \\
\hline $\mathbb{I P}, 13$ & $\langle A, R\rangle, 2$ \\
\hline$\aleph_{0}, 3$ & $\left\langle D, \leqslant^{*}\right\rangle, \bar{\jmath}$ \\
\hline$\aleph_{\alpha}, 3$ & $\langle X, \tau\rangle, \S$ \\
\hline$\beta \omega, 13$ & $\left\langle\alpha, \beta^{*}\right\rangle$-pré-gap, 52 \\
\hline $\mathcal{F} \perp \mathcal{G}, 36$ & $\left\langle\mathcal{P}(\omega), \subseteq^{*}\right\rangle, 5$ \\
\hline $\mathcal{F}\lceil A, 2$ & $\left\langle\mathcal{P}(\omega), \subset^{*}\right\rangle, 53$ \\
\hline $\mathcal{P}(A), 1$ & $\left\langle\omega_{\omega}^{\omega}, \leqslant^{*}\right\rangle, j$ \\
\hline$\chi(X), 15$ & $\left\langle{ }^{\omega} \omega,<^{*}\right\rangle, 53$ \\
\hline$\chi(p, X), 15$ & $\pi$-base, 10 \\
\hline
\end{tabular}


$Q, 13$

$\mathbb{R}, 13$

C, 5

$\tau, 8$

${ }^{A} B, 2$

$\omega_{\omega, 2}$

$c f(\beta), 4$

$f \leqslant g, 41$

$f \leqslant *^{*} g, 5$

$f \nmid A, 2$

$f[A], 2$

$f^{-1}[B], 2$

$f_{X}, 38$

$k c(X), 103$

$\min (A), 2$

$p \perp q, 46$

$\sup (A), 2$

$w(X), 15$

$x<{ }^{*} y, 5$

$\left\langle\alpha, \beta^{*}\right\rangle$-gap, 52

a.d., 16

aberto-fechado, 9

AC, 3

anticadeia, 46

Axioma de Martin, 45

axiomas

de enumerabilidade, 9

de separação, 9

big gap, 88

c.c.c., 46

c.u.b, 4 cadeia

em uma p.o., 46

caráter, 15

cardinal, 3

limite, 3

regular, 4

singular, 4

sucessor, 3

cardinalidade, 3

do espaço, 14

$\mathrm{CH}, 4$

clopen, 9

cofinal, 4

em $\mathcal{A} \subseteq[\omega]^{\omega}, 41$

em um ordinal, 4

em uma pré-ordem, 5

cofinalidade, 4

compatíveis, 46

completamente separados, 9

conjunto

ao lado do gap, 87

centrado em uma p.o., 47

concentrado, 105

magro, 10

raro, 10

conjunto-suporte, 8

consistência, 7

convergência

de seqüência, 11

de um conjunto, 11

denso 


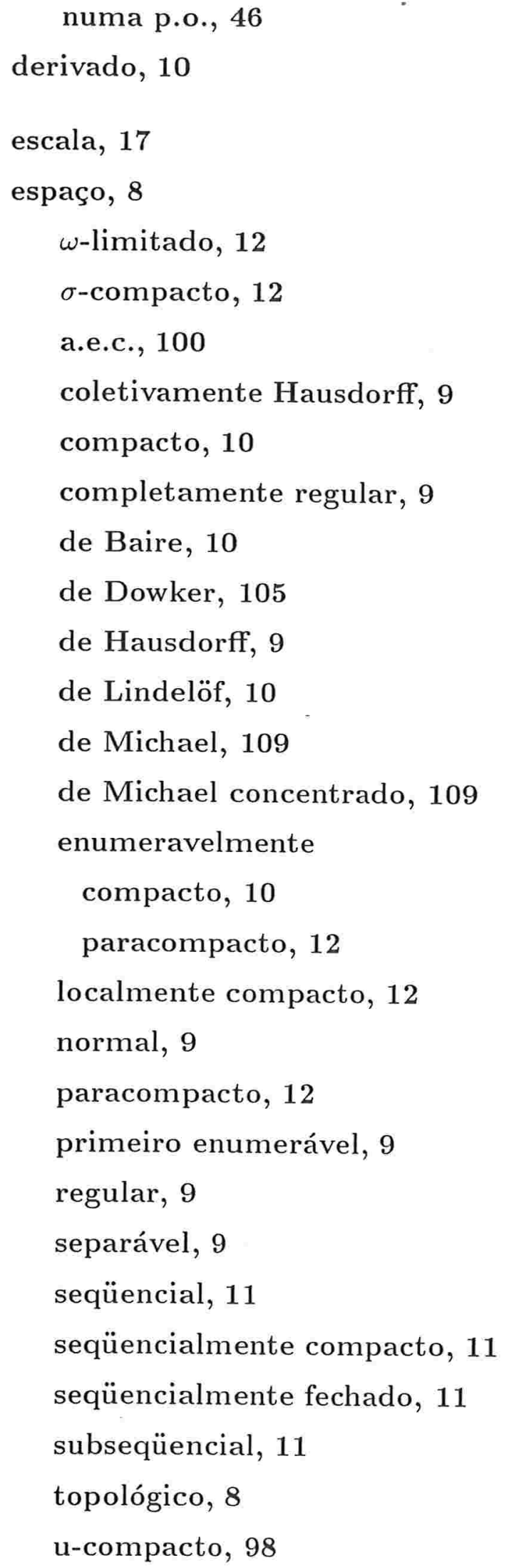


incompatíveis, 46

independência, 7

interpolador, 52

Lema

de Fodor, 5

de Solovay, 46

MA, 45

números naturais, 2

Open Problems, 15

ordinal, 2

limite, 2

regular, 4

singular, 4

sucessor, 2

P-ponto, 33

p.i.f, 7

p.o., 46

$\sigma$-centrada, 47

pequeno cardinal, iii

peso, 15

ponto

de acumulação completo, 10

de acumulação de seqüência, 11

pré-ordem, 5

de Rudin-Keisler, 14

propriedade

$\omega^{\omega}$ direcionada, 109

hereditária, 9

produtiva, 9

pseudo-intersecção, 17
R-espaços, 87

refinamento, 12

reta de Michael, 105

s.f.i.p., 17

s.p.g., 22

seqüência, 2

de comprimento $\alpha, 2$

diádica, 55

diádica dupla, 56

regular, 57

T-ponto, 98

Teorema, 8

da Completude, 7

de Cantor, 4

tight gap, 87

tightness, 15

tipo de ordem, 2

topologia

de subespaço, 9

da ordem, 9

produto, 9

torre, 17

u-limite, 97

ultrafiltro

não-principal ou livre, 7

principal, 7

sobre $X, 7$

ZFC, 1 